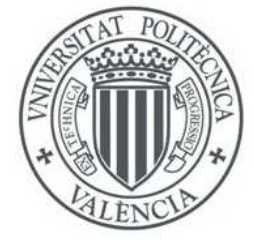

\title{
Formulación de un modelo de mecánica molecular aplicado al comportamiento estructural de los nanotubos de carbono
}

Tesis Doctoral presentada en el Departamento de Mecánica de los Medios Continuos y Teoría de Estructuras de la Universitat Politècnica de València

14 de junio de 2013

(c) Rafael Merli Gisbert

Autor: Rafael Merli Gisbert, ICCP Directores: Prof Dr. Salvador Monleón Cremades Prof Dr.Carlos Lázaro Fernández 

A mi madre, porque en sus ojos se refleja mi ilusión 



\section{Agradecimientos}

Escribir estas líneas significa que estoy muy cerca de alcanzar el sueño al que he dedicado mi vida en los últimos años. Finalizar esta tesis representa materializar mis ilusiones. Sin duda, debo mucho a las personas que me han acompañado en el camino y quiero agradecerles desde aquí su apoyo, su cariño y su dedicación.

En primer lugar, quiero agradecer a mi madre el incansable apoyo demostrado durante todo el proceso. Ella ha sabido hacerme reír ante los logros y no dejarme vencer por las frustraciones, siempre ha tenido tanta ilusión como yo en que llegara hasta aquí y me ha acompañado en el camino.

También mi hermano mayor, que hace muchos años me enseñó en gran medida cómo enfocar mis estudios, ha seguido mi evolución de cerca. Ha pasado bastante tiempo desde entonces, y sus consejos de Matemáticas, Física y programación siguen vigentes.

Quisiera agradecer a los directores de este trabajo la confianza depositada en mí y la dedicación mostrada. Por una parte, el Dr. Carlos Lázaro me ha impulsado enormemente en las publicaciones relacionadas con esta tesis, tanto en congresos como en revistas especializadas. Por otra, el Dr. Salvador Monleón ha mostrado un profundo respeto por mi trabajo, además de ayudarme a no flaquear en los momentos que éste parecía estar en una vía muerta. Aunque mis decisiones al recorrer algunas posibles líneas de investigación fueron equivocadas, ninguno de los dos ha dudado que el resultado final merecería la pena.

Asimismo, cabe señalar el apoyo mostrado por los Profesores Dr. José Casanova y Dr. Miguel Ángel Fernández, porque siempre me han transmitido una actitud positiva y han tenido tiempo para compartir alguna de mis dificultades en la investigación.

Quiero destacar también la presencia de amigos y compañeros de promoción (Kike, Javi, Lorenzo, Isma, Raúl, Tommy, Mayte...) que luego de tantos años siguen de cerca mis progresos. Gracias a todos ellos.

Siempre llevo en el corazón a mi abuelo materno, que confiaba ciegamente en mis éxitos. Todos los días me recordaba que algún día llegaría lejos. Estoy convencido que si pudiera leer estas líneas, sería completamente feliz.

Finalmente, quiero mencionar especialmente a D. Rafael Mauri Bonet, quien me confirmó que no es necesario tener una relación de sangre para ser familia, además de enseñarme a confiar en mi capacidad personal. 



\section{Resumen}

Desde su descubrimiento en 1991, los nanotubos de carbono han despertado un gran interés por parte de la comunidad científica e investigadora en todo el mundo. Esto es debido en gran medida a las excepcionales propiedades mecánicas, térmicas y eléctricas que presentan. Sus extraordinarias características les confieren prometedoras aplicaciones en el campo de la biomecánica, la industria aeronáutica y la ingeniería. En cualquier caso, la utilización extensiva de estos nuevos materiales en un futuro próximo pasa necesariamente por la mejora de los actuales procesos de fabricación, hasta la obtención de nanotubos de una forma económicamente rentable.

Una parte importante de la investigación relacionada con estas singulares moléculas se ha destinado a la predicción de su respuesta tenso-deformacional frente a acciones o estímulos externos. En algunos casos se han aplicado modelos ya existentes al comportamiento resistente de los nanotubos, mientras que en otros se han definido nuevos modelos con características propias. Como punto medio entre los costosos modelos de dinámica molecular y los modelos clásicos derivados de la mecánica del continuo, aparecen los modelos de mecánica estructural molecular (entre ellos, el stick-spiral), que permiten describir la estructura atómica del nanotubo a un coste computacional razonable.

Esta tesis doctoral tiene por objeto la formulación general del modelo stick-spiral y su aplicación a los nanotubos de carbono monocapa. Se ha explorado dicha formulación y sus resultados tanto en el marco del análisis geométricamente lineal como en el no lineal, además de contrastar las posibles diferencias en la respuesta estructural de los nanotubos debido a la consideración de dos potenciales interatómicos (AMBER y Morse).

En la primera parte del trabajo se ha estudiado la indeterminación estática y cinemática del modelo mediante una adaptación de la descomposición en valores singulares de Pellegrino y Calladine para estructuras articuladas, con el objetivo de averiguar la existencia de soluciones estáticamente admisibles. Este estudio permite establecer las ecuaciones cinemáticas y estáticas de cada elemento constituyente del modelo en el rango de la linealidad, así como plantear las características del ensamblaje de las matrices cinemáticas y de equilibrio en el modelo estructural completo. Este primer análisis ha puesto de manifiesto la verificación de la relación de contragradiencia para nuestro modelo y permite establecer la determinación cinemática del sistema en función de las condiciones de contorno presentes, así como la alta indeterminación estática interna que asegura la existencia de soluciones dada una determinada configuración de las cargas externas.

En una segunda etapa se aborda el análisis geométricamente lineal del modelo mediante una formulación en rigidez completamente general e independiente de la situación de carga mediante la aplicación sistemática de la ecuación de trabajos virtuales a los elementos estructurales, así como al sistema completo. Se proporcionan expresiones cerradas para las matrices de rigidez de cada elemento y se demuestra la verificación del ensamblaje booleano en el modelo. Adicionalmente, se presta especial atención a la curvatura inicial del nanotubo, incluyendo un sistema de esfuerzos iniciales (preeenergía o pretensado) que tiende a estabilizar la sección transversal y acortar el nanotubo. Como parámetros de contraste, se estiman las características mecánicas del nanotubo como conjunto bajo los esquemas de carga habituales en piezas alargadas (tracción, compresión, flexión y torsión), lo cual ha permitido validar la formulación comparando resultados con otros autores, como Natsuki y 
Endo; todo ello considerando dos potenciales interatómicos distintos, para averiguar la influencia de la no linealidad mecánica en la respuesta final. No obstante, se critica la validez de tales características mecánicas debido a su dependencia de ciertos parámetros geométricos como el espesor de pared equivalente, de determinación controvertida. Adicionalmente, se describen las ventajas del modelo de la tesis en relación a la dudosa aplicabilidad de los modelos continuos clásicos y respecto a los computacionalmente costosos métodos de dinámica molecular.

Posteriormente, se lleva a cabo el análisis del comportamiento de nanotubos frente a la inestabilidad desde un punto de vista geométricamente no lineal. El desarrollo de expresiones simbólicas para la matriz de rigidez tangente y la demostración del ensamblaje booleano en el caso no lineal ponen de manifiesto la potencia de la formulación en la obtención de deformaciones críticas con un esfuerzo computacional razonable. Desde el punto de vista numérico, se han implementado algoritmos incrementales-iterativos para cada uno de los potenciales interatómicos considerados, de forma que permitieron verificar que la no linealidad geométrica se desencadena previamente a la constitutiva, de acuerdo con Falvo et al. e Iijima et al. Como resultados se proporcionan las trayectorias de equilibrio no lineales, las deformaciones críticas bajo los esquemas de carga habituales en piezas alargadas y las geometrías deformadas al final del proceso de carga.

Finalmente, se estudia la adecuación de la geometría inicial a la proporcionada por el mapeo conforme sobre una superficie cilíndrica ideal. En concreto, se determinó el diámetro inicial del cilindro mediante la minimización de la energía total del sistema para las tres quiralidades, supuestos nulos los alargamientos de enlace respecto a la longitud de referencia sobre la lámina plana de grafeno. Los diámetros y esfuerzos obtenidos se contrastaron con los resultados del análisis lineal para el modelo de la tesis, hallando una íntima coincidencia entre los valores de salida de ambos procedimientos. La consideración de esta nueva geometría inicial representa una vía alternativa en la introducción de la curvatura del nanotubo en su comportamiento mecánico. 


\section{Resum}

Des de el seu descobriment en 1991, els nanotubs de Carbó han despertat un gran interés per banda de la comunitat científica e investigadora arreu el món. Això es debut en gran mesura a les excepcionals propietats mecàniques, térmiques y eléctriques que presenten. Les seues extraordinàries característiques els confereixen prometedores aplicacions al camp de la biomecànica, la industria aeronàutica y la enginyería. En qualsevol cas, la utilització extensiva d'aquestos nous materials en un futur próxim passa necessariament per la millora dels actuals processos de fabricació, fins l'obtenció de nanotubs d'una forma económicament rentable.

Una part important de la investigació relacionada amb estes singulars molécules ha sigut destinada a la predicció de la seua resposta tenso-deformacional davant d'accions o estímuls externs. En alguns casos, els models existents han sigut aplicats al comportament resitent dels nanotubs, mentres que en altres es defineixen nous models amb característiques pròpies. Com a punt mitjà entre els costosos models de dinàmica molecular i els models clàssics derivats de la mecànica del continu, apareixen els models de mecànica estructural molecular (entre ells, el stick-spiral), que permeten descriure l'estructura atómica del nanotub a un cost computacional raonable.

Aquesta tèsi doctoral té per objecte la formulació general del model stick-spiral i la seua aplicació als nanotubs de Carbó monocapa. S'ha explorat l'esmentada formulaciói els seus resultats tant al marc del l'anàlisi geométricament lineal com al no lineal, ademés de contrastar les possibles diferències en la resposta estructural dels nanotubs debut a la consideració de dos potencials interatómics (AMBER i Morse).

En la primera part del treball s'ha estudiat la indeterminació estàtica i cinemàtica del model mitjançant una adaptació de la descomposició en valors singulars de Pellegrino y Calladine per a estructures articulades, amb l'objectiu de trobar l'existència de solucions estàticament admissibles. Aquest estudi permet establir les equacions cinemàtiques i estàtiques de cada element constituent del model al camp de la linealitat, així com plantejar les característiques del muntatge de les matrius cinemàtiques i de equilibri dins del model estructural complet. Aquest primer anàlisi ha posat de relleu la verificació de la relació de contragradiència per al nostre model, i permet establir la determinació cinemàtica del sistema en funció de les condicions de contron existents, així com l'elevada indeterminació estàtica interna que assegura l'existència de solucions donada una configuració de les càrregues externes.

En una segona etapa s'adreça l'anàlisi geométricament lineal del model mitjançant una formulació en rigidesa completament general e independent de la situació de càrrega per mitjà de l'aplicació sistemàtica de l'ecuació de treballs virtuals als elements estructurals, així com al sistema complet. Es proporcionen expressions tancades per a les matrius de rigidesa de cada element i es demostra la verificació del muntatje booleà al model. Adicionalment, es presta especial atenció a la curvatura inicial del nanotub, incloent un sistema d'esforços inicials (preenergía o pretensat) que tendeix a estabilitzar la secció transversal i acurtar el nanotub. Com a parámetres de comparació, s'estimen les característiques mecàniques del nanotub com a conjunt davall dels esquemes de càrrega habituals en peçes allargades (tracció, compressió, flexió i torsió), lo qual ha permés validar la formulació comparant resultats amb altres autros, com ara Natsuki i Endo; tot plegat considerant dos potencials 
interatómics diferents, per a trobar l'influència de la no linealitat mecànica en la resposta final. No obstant aixó, es critica la validesa de les esmentades característiques mecàniques debut a la seua dependència de certs parámetres geométrics com ara el gruix de paret equivalent, de determinació controvertida. Adicionalment, es descriuen els avantages del model de la tèsi en front de la dubtosa aplicabilitat dels models continus clàssics i respecte als computacionalment costosos métodes de dinàmica molecular.

Més endavant, es du a terme l'anàlisi del comportament de nanotubs davant la inestabilitat des d'un punt de vista geométricament no lineal. El desenrollament d'expressions simbóliques per a la matriu de rigidesa tangent i la demostració del muntatge booleà al cas no lineal posen de relleu la potència de la formulació en l'obtenció de deformacións crítiques amb un esforç computacional raonable. Des del punt de vista numèric, s'han implementat algoritms incrementals-iteratius per a cada potencial interatómic considerat, de forma que permeteren verificar que la no linealitat geométrica es desenvolupa préviament a la constitutiva, d'acord amb Falvo et al. e Iijima et al. Com resultats es proporcionen les trajectòries d'equilibri no lineals, les deformacions crítiques davall els esquemes de càrrega habituals en peçes allargades i les geometríes deformades a la fi del procés de càrrega.

Finalment, s'estudía l'adecuació de la geometría inicial a la proporcionada pel mapeu conforme sobre una superfície cilíndrica ideal. En concret, es va determinar el diámetre inicial del cilindre mitjançant la minimització de l'energía total del sistema per a les tres quiralitats, suposats nuls els allargaments d'enllaç respecte a la longitud de referència sobre la lámina de grafit. Els diámetres i esforços obtinguts es varen contrastar amb els resultats de l'anàlisi lineal per al model de la tèsi, trobant una íntima coincidència entre els valors d'eixidda de tots dos procediments. La consideració d'esta nova geometría inicial representa una vía alternativa en la introducció de la curvatura del nanotub al seu comportament mecànic. 


\section{Abstract}

Since their discovery in 1991, carbon nanotubes have attracted great interest from the scientific and research community over the world. This is due in great measure to their exceptional mechanical, electrical and termical properties. Their outstanding features provide them promising applications in the framework of biomechanics, aircraft industry and engineering. Anyway, the broad use of these new materials in a near future requires necessarily some improvements at the grown procedures, up to the obtention of nanotubes in a economical way.

An important part of previous research works related to these singular molecules has been aimed to the prediction of their stress-strain response under external loading or excitations. In some cases, existing models have been applied to the strength behavior of nanotubes. Otherwise, new models with particular features have been defined. As a midpoint between the time-consuming methods from molecular dynamics and the classical models derived from the continuum mechanics, appear Molecular structural mechanics models (among them, the stick-spiral model) which allow to describe the atomistic structure of nanotubes at a reasonable computational cost.

This $\mathrm{Ph}$. Thesis is focused on the general formulation of the stick-spiral model and its application to single-walled carbon nanotubes. Such formulation and their results have been explored as much in the framework of the geometrical linear analysis as in the nonlinear one. Moreover, the possible differences in the structural response of nanotubes regarding two interatomic potentials (AMBER and Morse) have been compared.

In the first part of the work, the static and kinematic indetermination of the model have been studied by using and adaptation of the Single value decomposition from Pellegrino and Calladine for space trusses, with the target of finding out the existence of statically admisible solutions. This study allows to establish the kinematic and static equations for each constituent element in the linear range and to approach the assembling rules of the kinematic and equilibrium matrices for the whole structural model. This first analysis has underlined that contragradience relation is verified in our model, and let us to state the kinematic determination of the system in function of the existing boundary conditions. As well as the high internal static indetermination that ensures the existence of solutions from a given external loading configuration.

In the second part of the work, the geometrically linear analysis of the model is tackled through a fully general (independent of the loading configuration) stiffness formulation derived by means of the systematic application of the virtual work equation to the structural members and the complete system. Symbolic expressions of the stiffness matrices for each element are provided, as well as the verification of the boolean assembling is shown for the model. In addition, the initial curvature of nanotubes has been drawn especial attention, including a system of initial internal forces (preenergy of prestressing) which tend to stabilize the cross-section and to shorten the nanotube. As representative variables, the mechanical parameters of the nanotube subjected to the usual loading schemes used in beams or rods (tension, compression, bending and torsion) are estimated. Their numerical values have been used to validate our formulation compared to other authors, as Natsuki and Endo, regarding two different interatomic potentials in order to find out the effect of the constitutive nonlinearity in the final response. Nevertheless, the validity of such mechanical parameters is criticized due to their 
dependency on some geometrical parameters, as the controversial equivalent wall-thickness. Furthermore, the advantages of the presented model are described in contrast with the doubtful applicability of continuum models and the computationally expensive molecular dynamics methods.

Later on, buckling analysis of nanotubes is carried out from a geometrically nonlinear point of view. The development of symbolic expressions for the tangent stiffness matrix and the proof that the boolean assembling extends to the nonlinear case highlight the power of this formulation for the obtention of critical strains at a reasonable computational effort. From a numerical point of view, incremental-iterative procedures have been implemented for each interatomic potentials taken into account, in such a way they let us to verify that geometrical nonlinearity is triggered before the constitutive one, according to Falvo et al. and Iijima et al. As output results of our developed codes, nonlinear equilibrium paths, critical strains and final deformed shapes under the usual loading schemes in beam or rod structures are provided.

Finally, the suitability of the initial geometry provided from the conformal mapping on an ideal cylindrical surface is studied. Specifically, the initial diameter of the cylinder was determined by using the minimization of the total energy of the system under the three chiralities, assuming the bond lengthening vanish compared to the length of reference in the planar graphene sheet. The obtained diameter values and internal forces were compared with the results from the linear analysis of our model, finding a close agreement between the output values from both procedures. The consideration of this new initial geometry represents an alternative way to introduce the nanotube curvature into its mechanical behavior. 


\section{Índice general}

\begin{tabular}{|l|l}
\hline Agradecimientos & $\mathrm{V}$
\end{tabular}

Resumen vii

Resum ix

Abstract xi

$\begin{array}{lr}\text { 1. Introducción } & 1\end{array}$

1.1. Nanotubos de carbono: una presentación general . . . . . . . . . . . . . 1

1.1.1. Descripción geométrica . . . . . . . . . . . . . . . . . 1

1.1.2. Relación de los CNTs con otros materiales del carbono . . . . . . . . . . . . 4

1.1.3. Fabricación de nanotubos de carbono . . . . . . . . . . . . . . . . . 6

1.1.4. Desarrollo mediante CVD de nanotubos orientados . . . . . . . . . . . . . . 9

1.1.5. Aplicaciones de los Natotubos de carbono . . . . . . . . . . . . . . . . . 10

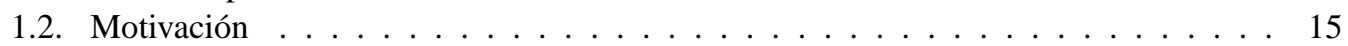

1.3. Objetivos de la tesis . . . . . . . . . . . . . . . . . . . . . . . . . . . . . . . . .

1.4. Contenido . . . . . . . . . . . . . . . . . . . . . 17

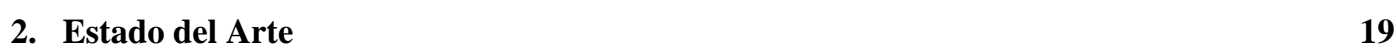

2.1. Clasificación esquemática de los métodos de análisis para CNTs . . . . . . . . . . . 19

2.2. Modelos de mecánica cuántica $(\mathrm{QM})$. . . . . . . . . . . . . . . . . 21

2.2.1. Método tight binding (TB) . . . . . . . . . . . . . . . 21

2.2.2. Métodos ab initio . . . . . . . . . . . . . . . . . . . . . . . . . . . . . . . 22

2.3. Modelos de dinámica molecular (MD) . . . . . . . . . . . . . . . . . 23

2.4. Modelos de mecánica estructural molecular (MSM) . . . . . . . . . . . . . . 25

2.4.1. Potenciales interatómicos . . . . . . . . . . . . . . . 26

2.5. Métodos de simulación multiescala (MS) . . . . . . . . . . . . . . . . . . . . 29

2.5.1. Métodos MS jerárquicos . . . . . . . . . . . . . . . . . . . . . . . . . . . . . . . . . . . . . . . .

2.5.2. Métodos MS simultáneos . . . . . . . . . . . . . . . . . 30

2.5.3. Condiciones de contorno multiescala $\ldots \ldots \ldots \ldots$. . . . . . . . . . . . . . . . .

2.6. Modelos híbridos atómico-continuos (HAC) . . . . . . . . . . . . . . . 31

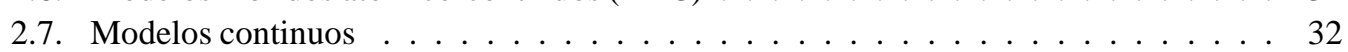

2.7.1. El modelo viga de Navien . . . . . . . . . . . . . . . . . . . . . . . . . . 32

2.7.2. El modelo pieza flexible 1D . . . . . . . . . . . . . . . 35

2.7.3. El modelo lámina . . . . . . . . . . . . . . . . . . . . . . . . . . . . . . . . . . 35

2.7.4. Método de los elementos finitos (MEF) . . . . . . . . . . . . . . . 36 
3. Caracterización estática y cinemática del modelo MSM 39

3.1. Descripción conceptual del modelo MSM stick-spiral . . . . . . . . . . . . . . . . . 39

3.2. Revisión abreviada de la formulación SVD . . . . . . . . . . . . . . . . . . . 40

3.2.1. Ecuaciones cinemáticas . . . . . . . . . . . . . . . . . 40

3.2.2. Ecuaciones de equilibrio . . . . . . . . . . . . . . . 41

3.2.3. Relación de contragradiencia . . . . . . . . . . . . . . . . . . 42

3.2.4. Descomposición en valores singulares (SVD) de la matriz de equilibrio . . . 42

3.3. Aplicación de la descomposición en valores singulares al modelo MSM . . . . . . . 43

3.3.1. Ecuaciones cinemáticas incluyendo distorsiones angulares . . . . . . . . . . 43

3.3.2. Ecuaciones de equilibrio incluyendo resortes angulares . . . . . . . . . . 47

3.3.3. Aplicación de las condiciones de contornd . . . . . . . . . . . . . . . 48

3.4. Resultados y discusión . . . . . . . . . . . . . . . . . . . . . . 49

4. Formulación general del modelo y su aplicación a nanotubos monocapa

4.1. Desarrollo energético de la formulación en rigidez . . . . . . . . . . . . . 53

4.1.1. Ecuaciones cinemáticas . . . . . . . . . . . . . . 53

4.1.2. Ecuaciones de equilibrio . . . . . . . . . . . . . . . . . . . 54

4.1.3. Ecuaciones constitutivas . . . . . . . . . . . . . . . 54

4.1.4. Formulación matricial en rigidez . . . . . . . . . . . . . . . . . . 57

4.1.5. El problema de la predeformación de SWNTs . . . . . . . . . . . . 60

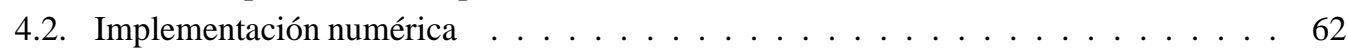

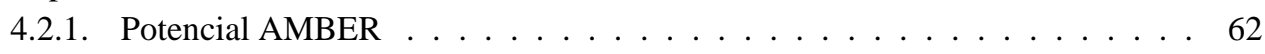

4.2.2. Potencial Morse . . . . . . . . . . . . . . . . . . . 63

4.3. Resultados y discusión . . . . . . . . . . . . . . . 65

4.3.1. Comportamiento a tracción . . . . . . . . . . . . . . . . 65

4.3.2. Comportamiento a compresión . . . . . . . . . . . . . . 73

4.3.3. Comportamiento a flexión . . . . . . . . . . . . . . . 78

4.3.4. Comportamiento a torsión . . . . . . . . . . . . . . . . . . 84

4.3.5. Deducción del coeficiente de Poisson . . . . . . . . . . . . . . 88

4.4. Sumario del análisis lineal . . . . . . . . . . . . . . . . . . . . . 90

5. Extensión de la formulación a la no linealidad geométrica 93

5.1. Ecuaciones de campo de la formulación no lineal . . . . . . . . . . . . . . . . 94

5.1.1. Ecuaciones cinemáticas . . . . . . . . . . . . . . . . . . . . 94

5.1.2. Ecuaciones de equilibrio . . . . . . . . . . . . . . . . . . . . 97

5.2. Ecuaciones constitutivas . . . . . . . . . . . . . . . . . . . . . 99

5.2.1. Matriz de rigidez tangente . . . . . . . . . . . . . . . 100

5.3. Implementación numérica $\ldots \ldots \ldots 103$

5.3.1. Planteamiento del problema numérico . . . . . . . . . . . . . . . . . . 104

5.3.2. Descripción del proceso incremental-iterativo . . . . . . . . . . . . . . 106

5.4. Resultados numéricos y discusión … . . . . . . . . . . . . . 108

5.4.1. Comportamiento a compresión . . . . . . . . . . . . . . . . . 108

5.4.2. Comportamiento a flexión . . . . . . . . . . . . . . . . 113

5.4.3. Comportamiento a torsión . . . . . . . . . . . . . . . . . . 120

5.5. Sumario del análisis geométricamente no lineal . . . . . . . . . . . . . . . 125 
6. Aproximación energética a la geometría inicial del nanotubo 127

6.1. Hipótesis iniciales y potenciales simplificados . . . . . . . . . . . . . . . . . . 127

6.2. Determinación del diámetro inicial para SWNTs ZigZag . . . . . . . . . . . . 128

6.2.1. Desarrollo con potencial AMBER . . . . . . . . . . . . . . . . . 130

6.2.2. Desarrollo con potencial Morse . . . . . . . . . . . . . . . . . 132

6.3. Determinación del diámetro inicial para SWNTs Armchair . . . . . . . . . . . . . 133

6.3.1. Desarrollo con potencial AMBER . . . . . . . . . . . . . . . . 136

6.3.2. Desarrollo con potencial Morse . . . . . . . . . . . . . . . . . . . 137

6.4. Determinación del diámetro inicial para SWNTs Chiral . . . . . . . . . . . . . . . . 139

6.4.1. Desarrollo con potencial AMBER . . . . . . . . . . . . . . . . . . 143

6.4.2. Desarrollo con potencial Morse . . . . . . . . . . . . . . . . . . . 146

6.5. Consideraciones sobre la coherencia de la formulación . . . . . . . . . . . . . 147

6.5.1. Comprobación Chiral-ZigZag . . . . . . . . . . . . . . . . . . . 147

6.5.2. Comprobación Chiral-Armchair . . . . . . . . . . . . . . . . . . 148

7. Conclusiones v líneas de investigación propuestas 151

7.1. Resumen del trabajo realizado . . . . . . . . . . . . . . . . . . 151

7.2. Conclusiones . . . . . . . . . . . . . . . . . . . . 152

7.2.1. En relación con la formulación del modelo . . . . . . . . . . . . . 152

7.2.2. En relación con los parámetros mecánicos equivalentes . . . . . . . . . . 153

7.2.3. En relación con la obtención de deformaciones críticas . . . . . . . . . . . 154

7.3. Aportaciones originales . . . . . . . . . . . . . . . . . . 155

7.4. Líneas de investigación propuestas . . . . . . . . . . . . . . . . . . . 156

7.4.1. Introducción del pretensado en el análisis geométricamente no lineal . . . . . 156

7.4.2. Deducción del mapeo generador de la geometría inicial . . . . . . . . . . . 158

7.4.3. Extensión de la formulación a la dinámica . . . . . . . . . . . . . . 158

7.4.4. Análisis estructural de MWNTs y nanosistemas . . . . . . . . . . . . . . 159

7.4.5. Introducción de la interacción con fluidos y otros entornos $\ldots \ldots \ldots$. . . 160

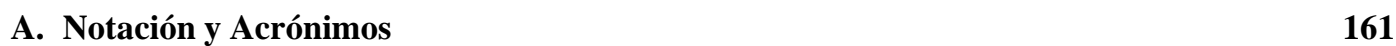

A.1. Notación . . . . . . . . . . . . . . . . . . . . . . . . . 161

A.2. Índice de acrónimos . . . . . . . . . . . . . . . . . . . . . . 167

\begin{tabular}{ll}
\hline Bibliografía & 176
\end{tabular} 



\section{Índice de figuras}

1.1. Definición geométrica célula unitaria $[26$, fig 2(a)] . . . . . . . . . . . . . . 2

1.2. Casos particulares de SWNTs dependiendo de la Quiralidad . . . . . . . . . . . . 3

1.3. Casos particulares de la hibridación $s p^{n}$ en átomos de C . . . . . . . . . . . . 4

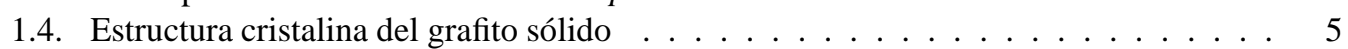

1.5. Método descarga de arco (esquema) . . . . . . . . . . . . . . . . . 7

1.6. Método la abrasión laser (esquema) . . . . . . . . . . . . . . . . . . . 8

1.7. Método deposición química de vapor (esquema) . . . . . . . . . . . . . . . . . 8

1.8. Generación orientada de MWNTs . . . . . . . . . . . . . . . . . . . . 9

2.1. Modelos de análisis estructural aplicados a CNTs . . . . . . . . . . . . . . . 20

3.1. Modelización adoptada para un CNT Zig-Zag . . . . . . . . . . . . . . . . 40

3.2. Definición vectores barra articulada $\ldots \ldots \ldots . \ldots \ldots$

3.3. Resorte angular genérico . . . . . . . . . . . . . . . . . . . . . 43

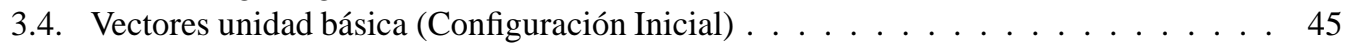

3.5. Esquema de orientaciones de barra . . . . . . . . . . . . . . . . . . 46

3.6. Geometría y apoyos $\mathrm{AC}(3,3), n_{L}=4.5$ celdas . . . . . . . . . . . . . . . . 50

3.7. Modos de mecanismo $\mathrm{AC}(3,3), n_{L}=4.5$ celdas $\ldots \ldots \ldots \ldots$

3.8. Ejemplos modos de autotensión $\mathrm{AC}(3,3), n_{I}=4.5$ celdas $\ldots \ldots \ldots$. . . . . . . 51

4.1. Rigideces tangentes Morse . . . . . . . . . . . . . . . . . . . . 57

4.2. Representación comparativa de las ecuaciones consitutivas . . . . . . . . . . . . 57

4.3. Esquema de cargas y apoyos en un SWNT . . . . . . . . . . . . . . . . . . . . 60

4.4. Diagrama de flujo no iterativo, potencial AMBER . . . . . . . . . . . . 63

4.5. Procedimiento iterativo para introducir el potencial Morse . . . . . . . . . . . . . 64

4.6. SWNTs Armchair y ZigZag a tracción, comparación con [77] y [78] . . . . . . . . 67

4.7. SWNTs Chiral a tracción, comparación con [15] . . . . . . . . . . . . . . 68

4.8. Variación del módulo de Young a tracción con el diámetro . . . . . . . . . . . . 70

4.9. Deformada ZZ(13,0) $\sigma_{x}=60 \mathrm{GPa}$ AMBER . . . . . . . . . . . . . . . . 71

4.10. Deformada AC(8,8) $\sigma_{r}=60 \mathrm{GPa}, \operatorname{Morse}(\mathrm{p}) \ldots \ldots \ldots \ldots$. . . . . . . . . 71

4.11. Deformada $\mathrm{CH}(8,5) \sigma_{x}=80 \mathrm{GPa}, \mathrm{AMBER} \ldots \ldots \ldots \ldots \ldots$. . . . . . . 71

4.12. Influencia de la quiralidad en el módulo de Young a tracción . . . . . . . . . . . 72

4.13. SWNTs Armchair y ZigZag a compresión, comparación con [77] y [78] . . . . . . . 74

4.14. SWNTs Chiral a compresión, comparación con [15] . . . . . . . . . . . . . 75

4.15. Variación del Módulo de Young a compresión con el diámetro . . . . . . . . . . . . 76

4.16. Deformada ZZ(11,0) $\sigma_{x}=60 \mathrm{GPa}$. AMBER . . . . . . . . . . . . . . . . 77

4.17. Deformada AC(7,7) $\sigma_{x}=60 \mathrm{GPa}$, Morse . . . . . . . . . . . . . . . 77

4.18. Deformada $\mathrm{CH}(10,6) \sigma_{x}=30 \mathrm{GPa}, \operatorname{AMBER}(\mathrm{p})$. . . . . . . . . . . . . . . . . 77 
4.19. SWNTs Armchair y ZigZag a flexión, comparación con $[78] \ldots$. . . . . . . . . . 79

4.20. SWNTs Chiral a flexión, comparación con $[15] \ldots \ldots$. . . . . . . . . . . . . 80

4.21. Variación del Módulo de Young a flexión con el diámetro . . . . . . . . . . . . . . 82

4.22. Algunas geometrías deformadas de SWNTs a flexión . . . . . . . . . . . . 83

4.23. SWNTs Armchair y ZigZag a torsión, comparación con $[15]$. . . . . . . . . . . . . 85

4.24. Variación del módulo de deformación transversal con el diámetro . . . . . . . . . . . 86

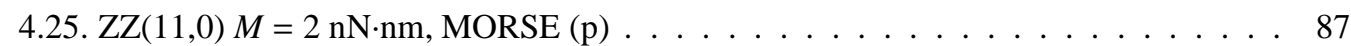

4.26. $\mathrm{AC}(7,7) M=3 \mathrm{nN} \cdot \mathrm{nm}, \mathrm{AMBER}(\mathrm{p}) \ldots \ldots \ldots \ldots$

4.27. $\mathrm{CH}(8,5) M=2 \mathrm{nN} \cdot \mathrm{nm}, \mathrm{AMBER}(\mathrm{p}) \ldots \ldots \ldots \ldots \ldots$

4.28. Variación del coeficiente de Poisson con el diámetro . . . . . . . . . . . . . . . 89

5.1. Esquema de la estrategia de control en cada incremento . . . . . . . . . . . . . . . . 104

5.2. Diagrama de flujo del proceso incremental-iterativo . . . . . . . . . . . . . . . . 106

5.3. Descomposición de un incremento genérico . . . . . . . . . . . . . . . . . . 107

5.4. Esquema de sustentación a compresión . . . . . . . . . . . . . . . . . . . . 109

5.5. Travectorias no lineales SWNTs compresión . . . . . . . . . . . . . . . . . 110

5.6. Deformadas finales SWNTs a compresión . . . . . . . . . . . . . . . . . 111

5.7. Esquema de sustentación a flexión . . . . . . . . . . . . . . . . . 114

5.8. Trayectorias no lineales SWNTs flexión . . . . . . . . . . . . . . . 116

5.9. Deformadas finales SWNTs a flexión . . . . . . . . . . . . . . . . . . 117

5.10. Proceso de deformación AC(4,4) AMBER a flexión . . . . . . . . . . . . . . . 119

5.11. Esquema de sustentación y desplazamientos impuestos a torsión . . . . . . . . . . 120

5.12. Travectorias no lineales SWNTs torsión . . . . . . . . . . . . . . . . . 122

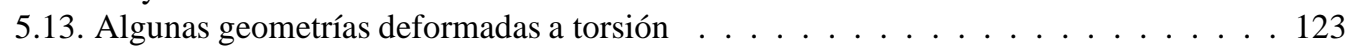

5.14. Proceso de deformación AC(4,4) AMBER a torsión . . . . . . . . . . . . . . . . . 124

6.1. Célula representativa SWNTs ZigZag . . . . . . . . . . . . . . . . . . . . 129

6.2. Esquemas vectoriales para obtener $\theta_{2}$. . . . . . . . . . . . . . . . . 129

6.3. Célula representativa SWNTs Armchair . . . . . . . . . . . . . . . . . . . . . . . . . . . . . . . . . . . .

6.4. Esquemas vectoriales para obtener $\theta_{1}, \theta_{2} \ldots \ldots \ldots$. . . . . . . . . . . . . 134

6.5. Orientaciones de enlace sistema elemental $\mathrm{CH}$. . . . . . . . . . . . . . . . 139

6.6. Notación enlaces sistema elemental CH . . . . . . . . . . . . . . . . . . 139

6.7. Esquema de deformación celda hexagonal tipo . . . . . . . . . . . . . . . . 140

6.8. Esquema vectorial enlaces $\mathrm{CH}$, perspectiva . . . . . . . . . . . . . . . 141

6.9. Esquema vectorial enlaces $\mathrm{CH}$, provecciones en sección . . . . . . . . . . . . . . 142

6.10. Diagrama de flujo del proceso iterativo para la obtención de $d_{0} \ldots$. . . . . . . . . . 145

6.11. Definición de parámetros formulaciones Chiral y Armchair . . . . . . . . . . . . 149

7.1. Diagrama de flujo incremento cero . . . . . . . . . . . . . . . . . . 157

7.2. Incremento $(n=0)$, estrategia de control de fuerza $\ldots \ldots \ldots 157$ 


\section{Índice de tablas}

3.1. Resultados tipo descomposición SVD . . . . . . . . . . . . . 50

4.1. Resumen de las ecuaciones cinemáticas . . . . . . . . . . . . . . . . . 53

4.2. Resumen de las ecuaciones de equilibrio . . . . . . . . . . . . . . . . . . . 54

4.3. Dimensiones y quiralidades de los SWNTs ensayados . . . . . . . . . . . . . . 65

4.4. Cargas puntuales aplicadas en simulaciones a tracción . . . . . . . . . . . . . . 66

4.5. Módulo de Young equivalente a tracción . . . . . . . . . . . . . . . . . . 69

4.6. Dimensiones y quiralidades de los SWNTs adicionales a tracción . . . . . . . . . . 72

4.7. Módulo de Young a tracción frente a quiralidad . . . . . . . . . . . . . . . . 72

4.8. Cargas puntuales aplicadas en simulaciones a compresión . . . . . . . . . . . . 73

4.9. Módulo de Young equivalente a compresión . . . . . . . . . . . . . . . . 76

4.10. Momentos externos de flexión (nN.nm) aplicados en el extremo libre . . . . . . . . . 78

4.11. Cargas puntuales aplicadas en flexión para $M_{z}=1 \mathrm{nN} \cdot \mathrm{nm}$. . . . . . . . . . . 78

4.12. Módulo de Young equivalente a flexión . . . . . . . . . . . . . . . . . 81

4.13. Cargas aplicadas a torsión para $M_{t}=1 \mathrm{nN} \cdot \mathrm{nm} \ldots \ldots \ldots \ldots$. . . . . . . . . 84

4.14. Módulo de deformación transversal de equivalente a torsión . . . . . . . . . . . 86

4.15. Coeficiente de Poisson a partir de tracción y torsión . . . . . . . . . . . . . . . 89

5.1. SWNTs ensayados a compresión . . . . . . . . . . . . . . . . . . 109

5.2. Deformaciones críticas a compresión . . . . . . . . . . . . . . . . 111

5.3. Datos de entrada en simulaciones a compresión . . . . . . . . . . . . . . . 112

5.4. Deformaciones críticas de compresión con simulaciones MD . . . . . . . . . . . 112

5.5. Comparación con Yakobson et al. 115, eq. (2)] a compresión . . . . . . . . . . . 113

5.6. SWNTs ensayados a flexión . . . . . . . . . . . . . . . . 113

5.7. Curvaturas críticas de abolladura a flexión . . . . . . . . . . . . . . . . . 117

5.8. Datos de entrada en simulaciones a flexión . . . . . . . . . . . . . . . . . 118

5.9. Curvaturas críticas de abolladura a flexión estimadas según [115, eq. (4) . . . . . . 118

5.10. SWNTs ensayados a torsión . . . . . . . . . . . . . . . . 120

5.11. Datos de entrada en simulaciones a torsión . . . . . . . . . . . . . . . . . . . . 123

5.12. Rotaciones críticas de abolladura a torsión . . . . . . . . . . . . . . 125

6.1. Potenciales interatómicos . . . . . . . . . . . . . . . . . . . 128

6.2. Diámetros iniciales ZigZag. AMBER . . . . . . . . . . . . . . . . 131

6.3. Esfuerzos minimización energética ZZ, AMBER . . . . . . . . . . . . . 131

6.4. Esfuerzos modelo MSM (ZZ análisis lineal, AMBER) . . . . . . . . . . . . . 131

6.5. Errores relativos respecto a la minimización energética ZZ, AMBER . . . . . . . . 132

6.6. Diámetros iniciales ZigZag, Morse . . . . . . . . . . . . . . . . . 132

6.7. Esfuerzos minimización energética ZZ, Morse . . . . . . . . . . . . . . . . . 133 
6.8. Esfuerzos modelo MSM (ZZ análisis lineal, Morse) . . . . . . . . . . . . . . . . . 133

6.9. Errores relativos respecto a la minimización energética ZZ, Morse . . . . . . . . . 133

6.10. Diámetros iniciales Armchair, AMBER . . . . . . . . . . . . . . . 136

6.11. Esfuerzos minimización energética AC, AMBER . . . . . . . . . . . . . 136

6.12. Esfuerzos modelo MSM (AC análisis lineal, AMBER) . . . . . . . . . . . . . . 137

6.13. Errores relativos respecto a la minimización energética AC, AMBER . . . . . . . . 137

6.14. Diámetros iniciales Armchair, Morse . . . . . . . . . . . . . . . . 138

6.15. Esfuerzos minimización energética AC, Morse . . . . . . . . . . . . . . . . 138

6.16. Esfuerzos modelo MSM (AC análisis lineal, Morse) . . . . . . . . . . . . . 138

6.17. Errores relativos respecto a la minimización energética AC, Morse . . . . . . . . . 138

6.18. Diámetros iniciales Chiral, AMBER . . . . . . . . . . . . . . . . . . . . 144

6.19. Esfuerzos minimización energética CH, AMBER . . . . . . . . . . . . . . . . . 144

6.20. Esfuerzos modelo MSM (CH análisis lineal, AMBER) . . . . . . . . . . . . . . 144

6.21. Errores relativos respecto a la minimización energética $\mathrm{CH}, \mathrm{AMBER}$. . . . . . . . 145

6.22. Diámetros iniciales Chiral, Morse . . . . . . . . . . . . . . . . . 146

6.23. Esfuerzos minimización energética CH, Morse . . . . . . . . . . . . . . . . . . 146

6.24. Esfuerzos modelo MSM (CH análisis lineal, Morse) . . . . . . . . . . . . . . . 147

6.25. Errores relativos respecto a la minimización energética $\mathrm{CH}$, Morse . . . . . . . . . 147

6.26. Comparación diámetros obtenidos Chiral-ZigZag. AMBER . . . . . . . . . . . . . 148

6.27. Comparación diámetros obtenidos Chiral-Armchair, AMBER . . . . . . . . . . . 150

A.1. Variables que definen la geometría de los SWNTs . . . . . . . . . . . . . . . . 161

A.2. Variables asociadas a los elementos barra del modelo . . . . . . . . . . . . . . . 161

A.3. Variables asociadas a los elementos muelle del modelo . . . . . . . . . . . . . 162

A.4. Variables cinemáticas (análisis lineal) asociadas al sistema estructural completo . . . 162

A.5. Variables estáticas (análisis lineal) asociadas al sistema estructural completo . . . . . 162

A.6. Magnitudes y parámetros incluidas en las ecuaciones constitutivas . . . . . . . . . 163

A.7. Magnitudes incluidas en la formulación en rigidez, análisis lineal geométrico . . . 163

A.8. Variables cinemáticas, análisis no lineal geométrico . . . . . . . . . . . . . . 164

A.9. Variables estáticas, análisis no lineal geométrico . . . . . . . . . . . . . . . . 164

A.10. Magnitudes ecs. constitutivas incrementales, análisis no lineal geométrico . . . . . 164

A.11. Variables sistema incremental de rigidez, análisis no lineal geométrico . . . . . . . 165

A.12.Determinación diámetro inicial por minimización energética, magnitudes comunes . 165

A.13.Determinación diámetro inicial por minimización energética, SWNTs ZigZag . . . . 165

A.14. Determinación diámetro inicial por minimización energética, SWNTs Armchair . . . 166

A.15.Determinación diámetro inicial por minimización energética, SWNTs Chiral . . . . . 166 


\section{Capítulo 1}

\section{Introducción}

Desde su descubrimiento en 1991 por Iijima [42], los nanotubos de carbono han despertado un gran interés por parte de la comunidad científica e investigadora en todo el mundo. Esto es debido en gran medida a las excepcionales propiedades mecánicas, térmicas y eléctricas que presentan. En particular, dentro del marco de la Ciencia de Materiales y de la Ingeniería Civil, los composites reforzados con nanotubos de carbono así como los polímeros reforzados con nanopartículas han mostrado una amplia gama de aplicaciones potenciales.

Los nanotubos de carbono presentan un singular acoplamiento entre las propiedades eléctricas y la deformación mecánica [83], convirtiéndose en los candidatos ideales para construir sensores a escala nano y sistemas nano-electromecánicos, de prometedoras aplicaciones en el campo de la Biomecánica. Además tienen la capacidad de comportarse eléctricamente como metales o semiconductores, dependiendo de la orientación de los enlaces $\mathrm{C}-\mathrm{C}$, incluso se pueden considerar transistores 500 veces más pequeños que los dispositivos actuales.

Las propiedades mecánicas más interesantes en cuanto a sus aplicaciones como materiales estructurales son su extremada rigidez longitudinal (módulos de Young del orden de $E \simeq 1 \mathrm{TPa}$ ) y su elevada resistencia a tracción $\left(\sigma_{t}=50 \mathrm{GPa}\right)$. En cualquier caso, la utilización extensiva de estos nuevos materiales en un futuro próximo pasa necesariamente por la mejora de los actuales procesos de fabricación, hasta la obtención de nanotubos con un nivel mínimo de defectos estructurales y de una forma económicamente rentable.

\subsection{Nanotubos de carbono: una presentación general}

Para facilitar la lectura de la tesis se describen a continuación las principales características físicas de los nanotubos de carbono, así como de algunos materiales relacionados con ellos. Se resumen además los actuales métodos de fabricación y las aplicaciones de estas moléculas. La mayor parte del contenido de esta sección sigue lo desarrollado en [26].

\subsubsection{Descripción geométrica}

Los nanotubos de carbono (CNTs) son moléculas de geometría cilíndrica formadas a partir de una lámina de grafeno con entramado de panal de abeja enrollada sobre sí misma sin costuras. Los nanotubos de carbono monocapa (SWNTs) están formados por una única lámina, mientras que los multicapa (MWNTs) consisten en varias láminas de grafeno dispuestas en superficies cilíndricas concéntricas aproximadamente equidistantes entre sí. 
La estructura de un SWNT se define en términos de su célula unitaria 2D (figura 2.1), cuya geometría viene determinada por los siguientes vectores:

$\square$ Vectores base $\left(\mathbf{a}_{1}, \mathbf{a}_{2}\right)$ del entramado hexagonal de la lámina

$\square$ Vector quiral (o chiral) $\mathbf{C}_{h}=n \mathbf{a}_{1}+m \mathbf{a}_{2}$, conecta dos localizaciones cristalográficamente equivalentes en la lámina y determina la longitud de la circunferencia del cilindro que forma el SWNT. La pareja de enteros $(n, m)$ son los números de celdas hexagonales en las respectivas direcciones unitarias.

$\square$ Vector traslación $(\mathbf{T})$, determinado por la intersección de la perpendicular al vector $\mathbf{C}_{h}$ y la malla hexagonal. Es paralelo al eje del SWNT.

(a)

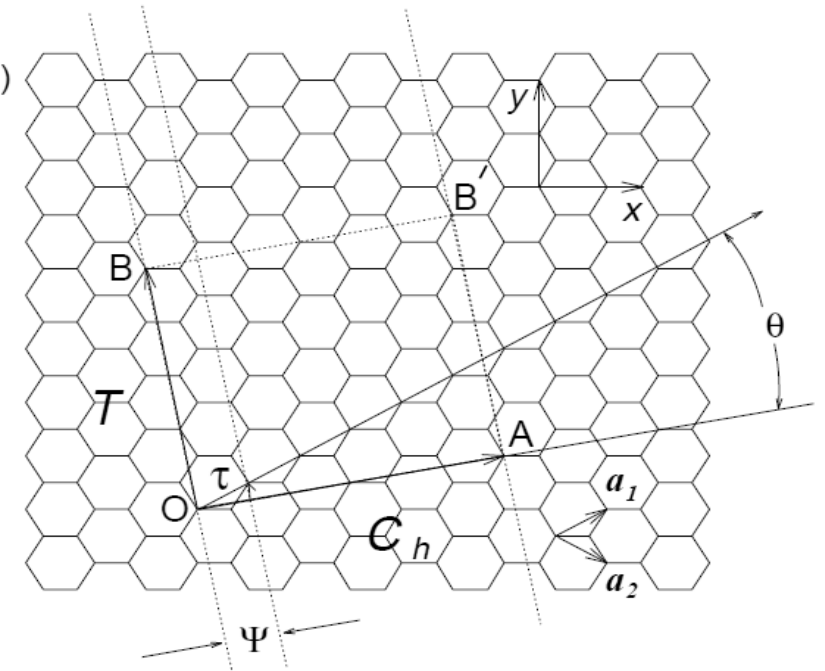

Figura 1.1: Definición geométrica célula unitaria [26 fig 2(a)]

Suponiendo que los átomos de C están situados en la superficie cilíndrica una vez enrollada la lámina de grafeno y que los enlaces covalentes se disponen según las secantes a tal superficie (mapeo conforme), podemos calcular el diámetro del SWNT en función de los valores $(n, m)$ como:

$$
D_{t}=\frac{\sqrt{3} a_{0}}{\pi} \sqrt{n^{2}+m n+m^{2}}
$$

donde $a_{0}$ es la longitud del enlace covalente C-C, estimada habitualmente en $a_{0}=1.42 \AA$.

Llamando dirección ZigZag a la definida por el vector unitario $\mathbf{a}_{1}$, el ángulo quiral $\theta$ será el comprendido por ésta y el vector quiral $\mathbf{C}_{h}$, y se puede calcular 1 en función de los enteros $(n, m)$ como:

$$
\theta=\arctan \frac{\sqrt{3} m}{2 n+m}
$$

dando lugar a tres casos particulares de nanotubos (fig. 1.2) dependiendo de la orientación del entramado respecto al eje del SWNT: 1]

${ }^{1}$ Las expresiones de la mayor parte de variables implicadas en la geometría de los SWNTs puede consultarse en [26] Tabla 
$\square$ ZigZag $(\mathrm{n}, 0), \theta=0$.- Un tercio de los enlaces covalentes tienen una orientación paralela al eje del SWNT.

$\square$ Armchair (n,n), $\theta=30^{\circ}$.- Un tercio de los enlaces covalentes tienen una orientación perpendicular al eje del SWNT.

$\square$ Chiral (n,m), $0<\theta<30^{\circ}$.- Todos los enlaces covalentes tienen una orientación oblicua respecto al eje del SWNT.

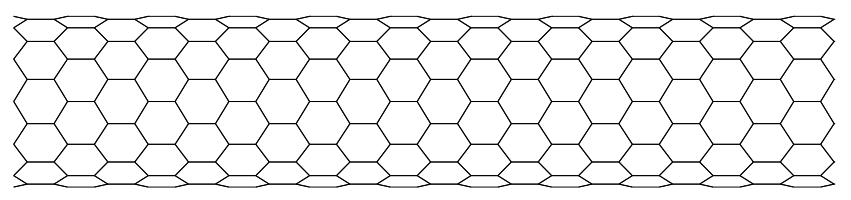

(a) $\operatorname{ZigZag}(\mathrm{n}, 0) \theta=0$

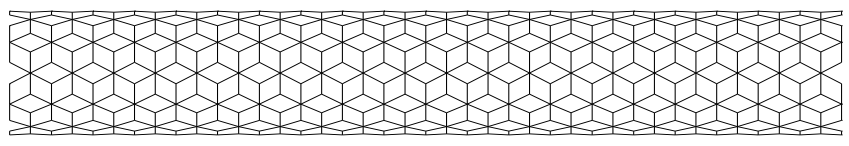

(b) Armchair(n,n) $\theta=30^{\circ}$

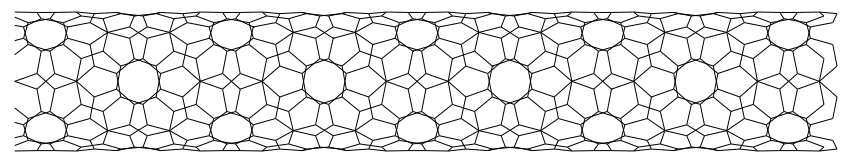

(c) $\operatorname{Chiral}(\mathrm{n}, \mathrm{m}) 0<\theta<30^{\circ}$

Figura 1.2: Casos particulares de SWNTs dependiendo de la Quiralidad

Los SWNTs ZigZag y Armchair son considerados aquirales y poseen simetría cilíndrica respecto al eje del nanotubo, entendida esta como la invarianza cristalográfica que se produce en el SWNT al dotar al entramado de una rotación según el eje, de ángulo $\psi$ equivalente a una celda hexagonal. Para el caso más general de los SWNTs Chiral, el vector simetría $\mathbf{R}(\psi \mid \tau)$ ( $\tau$ define la traslación unitaria o pendiente) del grupo simétrico representa una transformación de la malla en otra con idéntico estado cristalográfico, pero desplazada una celda sobre la dirección ZigZag $(\theta=0)$; diremos entonces que los SWNTs Chiral poseen simetría helicoidal.

Los MWNTs están constituidos por varias láminas cilíndricas concéntricas de entramados hexagonales con diferentes orientaciones y distribuciones, con la única restricción experimental de que la separación entre capas sea prácticamente igual a la separación entre láminas hexagonales en el grafito sólido $t=0.335 \mathrm{~nm}$. 
Los CNTs aparecen cerrados en sus extremos por cúpulas de fullerenos 2 (láminas semiesféricas que pueden contener hexágonos, pentágonos y heptágonos) que deben verificar la Regla del pentágono aislado. Esta regla requiere que no se localicen dos pentágonos adyacentes entre sí, ya que en caso contrario la energía de deformación de la cúpula no sería mínima [26, cap. 1].

\subsubsection{Relación de los CNTs con otros materiales del carbono}

\section{Química del enlace C-C}

El carbono es el sexto elemento de la tabla periódica y tiene el menor número atómico en la columna 14 (grupo IVA). Cada átomo de $\mathrm{C}$ tiene 6 electrones con una estructura electrónica $1 s^{2}, 2 s^{2}, 2 p^{2}$. El orbital $1 s^{2}$ contiene dos electrones fuertemente enlazados al núcleo, mientras que los electrones de valencia se encuentran en los orbitales $2 s^{2}, 2 p^{2}$, siendo los responsables de formar enlaces covalentes en materiales carbónicos.

La hibridación $s p^{n}$ con $n=1,2,3$ es la combinación general de orbitales $2 s$ y $2 p$, con las distribuciones geométricas representadas en la figura 1.3. La hibridación $s p$ da lugar a estructuras de enlace tipo cadena, la $s p^{2}$ a estructuras planas y la $s p^{3}$ a estructuras espaciales tetraédricas.

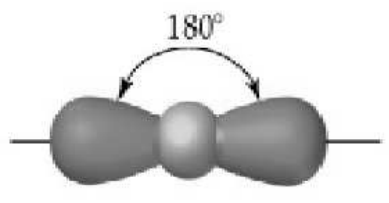

(a) Hibridación $s p$

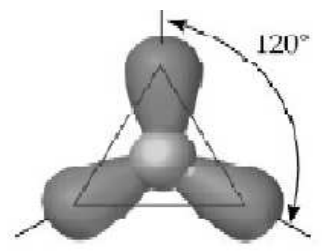

(b) Hibridación $s p^{2}$

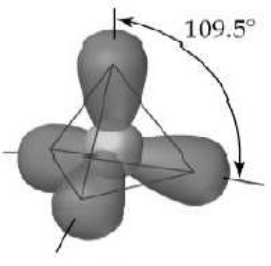

(c) Hibridación $s p^{3}$

Figura 1.3: Casos particulares de la hibridación $s p^{n}$ en átomos de $C$

El grafito $s p^{2}$ es el estado natural del carbono en condiciones ambientales, pero a elevadas presiones y temperaturas, el diamante cúbico $s p^{2}$ es estable. La razón física por la que se forman CNTs es que una lámina de grafeno 2D de tamaño finito tiene muchos átomos de borde con enlaces oscilantes, que corresponden con altos niveles de energía. Por tanto, la energía total de un pequeño $\mathrm{n}^{\mathrm{O}}$ de átomos de C (30-100) se reduce eliminando dichos enlaces de borde, incluso a costa de un incremento de la energía de deformación, formándose bloques cerrados como los fullerenos o los nanotubos.

Aunque los CNTs están íntimamente relacionados con la lámina de grafeno 2D, la curvatura del tubo y el confinamiento atómico en la dirección circunferencial producen muchas propiedades diferentes a las de la lámina.

\section{Grafito}

La estructura cristalina ideal del grafito (figura 1.4 está formada por capas en las cuales los átomos de C están dispuestos en los vértices y los enlaces covalentes C-C en las aristas de una red abierta con entramado hexagonal. Dichas láminas se disponen paralelamente, aunque parcialmente decaladas entre sí. La disposición habitual (conocida como apilado Bernal o ABAB) tiene una longitud de enlace C-C $a_{0}=1.421 \AA$ y una separación interplanar de $c_{0} / 2=3.354 \AA$.

Puesto que los enlaces covalentes C-C son muy fuertes y la distancia $a_{0}$ muy reducida, el entramado plano es muy estable frente a perturbaciones externas. En consecuencia, es muy improbable que alguna especie impura se aloje en las localizaciones del entramado hexagonal por sustitución y

\footnotetext{
${ }^{2}$ Llamados así en honor a Richard Buckminster Fuller, Arquitecto e Ingeniero estadounidense famoso por sus proyectos de cúpulas geodésicas
} 
bastante más lógico que ocupe alguna posición intersticial entre las láminas de grafeno (compuestos de intercalación), que están relacionadas mediante una débil interacción de Van der Waals (VDW).

La conductividad eléctrica de los compuestos de intercalación ocurre sobre todo en las capas de grafeno como resultado de la transferencia de electrones entre las especies intercaladas y las capas matriz. Se aplican actualmente en las baterías de litio para móviles. Los CNTs también proporcionan un material matriz para el alojamiento de especies externas tanto en la cara exterior como en los núcleos huecos de los SWNTs, por tanto se espera poder aplicarlos en baterías de muy alta capacidad.

Con frecuencia, las capas de grafeno tienen defectos de apilado que se traducen en un pequeño incremento de la distancia interplanar desde $3.354 \AA$ hasta un valor de $3.440 \AA$. Cuando se alcanza esta distancia, las capas individuales de grafeno se independizan sin

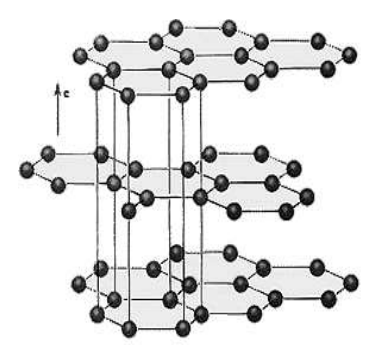

Figura 1.4: Estructura cristalina del grafito sólido ninguna relación en las localizaciones entre átomos de $\mathrm{C}$ de dos capas diferentes, cuyo resultado se llama grafito turboestratificado. Debido a los diferentes diámetros entre las capas de MWNTs, la disposición estructural de los entramados hexagonales de dos capas adyacentes es esencialmente independiente sin ninguna correlación entre dos átomos de $\mathrm{C}$ situados cada uno en una capa, provocando una gran facilidad de deslizamiento entre capas (tipo de fallo espada-vaina a tracción).

\section{Filamentos de carbono}

Los filamentos de carbono son un material grafítico formado enrollando una lámina de grafeno en un ovillo. Existen muy pocas referencias relacionadas con este material. El diámetro varía en el rango $1-5 \mu \mathrm{m}$ y pueden llegar a tener $3 \mathrm{~cm}$ de largo.

Estos filamentos muestran una gran perfección cristalina, alta conductividad eléctrica y un elevado módulo de Young longitudinal. Además, sirvieron como punto de referencia para la fabricación de fibras de carbono.

La fabricación de filamentos de carbono por el método del arco eléctrico tiene muchas similitudes con la fabricación de MWNTs, aunque la presión de gas comúnmente empleada para los filamentos es bastante más alta.

\section{Fibras de carbono}

Las fibras de carbono generadas por deposición de vapor (VGCF) tienen una morfología tipo "piel de cebolla" o "tronco de árbol", y tras un tratamiento térmico de unos $2500^{\circ} \mathrm{C}$ se asemejan mucho a los CNTs. En el núcleo hueco de una fibra de carbono se suele alojar un MWNT (incluso un SWNT), que puede observarse una vez fracturada la fibra.

Las excelentes propiedades mecánicas de los CNTs están íntimamente relacionadas con las de las fibras de carbono, aunque podemos encontrar algunas diferencias, tales como la flexibilidad de los SWNTs (frente a la flexión) y el buen comportamiento a compresión de los MWNTs (con sólo unas pocas capas), en contraste con las fibras de carbono que presentan una elevada fragilidad a compresión.

Las fibras de carbono se pueden presentar en un amplio rango de diámetros (desde $\simeq 10 \mathrm{~nm}$ hasta más de $100 \mu \mathrm{m}$ ) y tienen núcleos centrales huecos. Se debe hacer una distinción entre las fibras VGCF y las fibras en el rango de 10-100 $\mu$ m, llamadas nanofibras, que muestran propiedades intermedias entre las fibras VGCF y los MWNTs. Kharissova [49] afirma haber fabricado CNTs con un microondas doméstico, empleando grafito en polvo y un catalizador metálico, pero por los diámetros obtenidos (168-400 nm), ha obtenido fibras de carbono o nanofibras.

Las fibras comerciales disponibles actualmente se pueden clasificar en dos grupos: 
$\square$ Fibras basadas en brea de petróleo.- presentan un elevado módulo de Young debido a un alto grado de orientación de las capas de grafeno adyacentes en la dirección longitudinal a la fibra, además de una importante conductividad térmica.

$\square$ Poliacrilonitrilo (PAN).- su empleo está ampliamente extendido por su elevada resistencia a tracción, causada porque los defectos existentes en la estructura inhiben el deslizamiento entre capas de grafeno adyacentes (fallo tipo espada-vaina).

Los diámetros habituales de las fibras de carbono comerciales se sitúan en los $7 \mu \mathrm{m}$ y pueden llegar a ser muy largas. Su módulo de Young suele estar en torno a $E=207 \mathrm{GPa}$ y su resistencia a tracción $f_{y}=1.4 \mathrm{GPa}$.

\section{Carbono líquido}

El carbono líquido a presión atmosférica sólo es estable a muy altas temperaturas (punto de fusión del grafito $\simeq 4450^{\circ} \mathrm{K}$ ). Se ha producido en laboratorio mediante fusión láser del grafito o por calentamiento mediante resistencias.

Aunque el diamante y el grafito tienen diferentes puntos de fusión, se cree que fundiendo ambos se obtiene el mismo carbono líquido. Incluso es probable que la fusión de CNTs también produzca carbono líquido. Consideraciones energéticas sugieren que las especies emitidas desde una superficie de carbono fundido tienen masas comparables con los fullerenos [26].

La resistencia eléctrica de las fibras de carbono bien grafitizadas crece más o menos linealmente con la temperatura, hasta el punto de fusión, donde decrece bruscamente un orden de magnitud. Por tanto, el carbono líquido posee una gran conductividad eléctrica.

\subsubsection{Fabricación de nanotubos de carbono}

En los últimos años se ha llevado a cabo un intenso esfuerzo en la mejora y optimización de los procesos de fabricación de CNTs. Sin embargo, muchos retos permanecen sin resolver en este campo:

$\square$ Falta un método de fabricación de CNTs estructuralmente perfectos a gran escala

$\square$ Fabricar nanotubos libres de defectos hasta longitudes macroscópicas ha resultado difícil hasta la fecha

$\square$ Se necesita aprender a controlar la generación de CNTs sobre superficies y obtener nanoestructuras ordenadas

$\square$ Falta controlar la quiralidad de SWNTs a través de algún método de fabricación

Describimos a continuación los métodos que se vienen empleando con un éxito relativo en la obtención de CNTs.

\section{Método de la descarga de arco}

Este método y la erosión láser incluyen la condensación de átomos de carbono generados a partir de la evaporación de fuentes de carbono sólido. En este caso, la evaporación se produce en un plasma de helio inflamado por altas corrientes que se establecen entre ánodo y cátodo de carbono opuestos.

Por este método se obtienen SWNTs y MWNTs de gran calidad. Para la obtención de SWNTs se requiere disponer un metal catalizador en el ánodo de carbono (en ocasiones cobalto, o bien una 


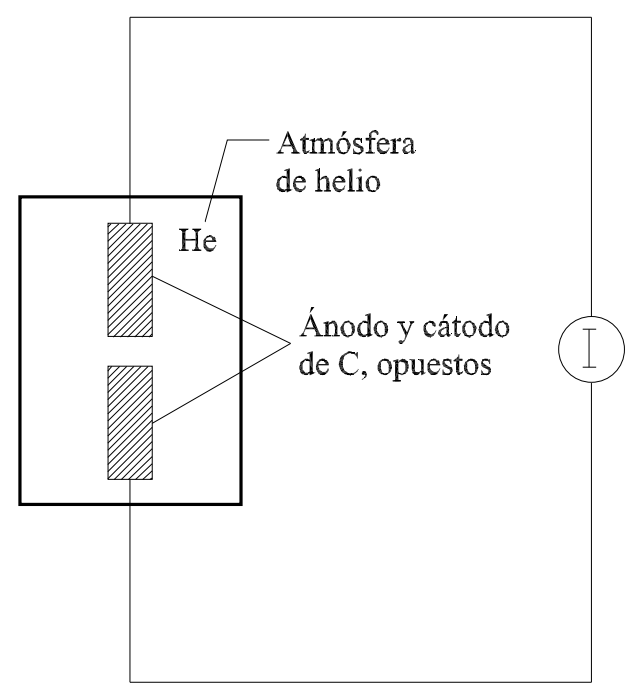

Figura 1.5: Método descarga de arco (esquema)

mezcla de ytrio y níquel). Por otra parte, se pueden obtener diferentes MWNTs regulando las condiciones de fabricación (presión del gas, intensidad de corriente, temperatura), obteniendo diámetros $D_{t} \simeq 5-30 \mathrm{~nm}$ y longitudes de $10 \mu \mathrm{m}$.

Los MWNTs están normalmente unidos por interacciones de Van der Waals formando fajos prietos de nanotubos muy rectos, lo cual indica su alta cristalinidad. Ocasionalmente, aparecen defectos estructurales tales como pentágonos o heptágonos en las paredes de los nanotubos.

En el caso de SWNTs aparecen como subproductos del proceso: fullerenos, partículas poliédricas de grafito con partículas de metal encerradas, y carbono amorfo abrazando las paredes laterales del nanotubo. Estos subproductos se pueden reducir o eliminar sometiendo a los SWNTs a un proceso de purificación, basado en un reflujo del material en ácido nítrico durante un largo periodo de tiempo, oxidando el carbono amorfo y eliminando algunas especies de material catalizador.

Los subproductos de la fabricación de MWNTs son partículas poliédricas multicapa de grafito, cuya purificación se puede conseguir calentando el material producido en una atmósfera oxidante, ya que las partículas poliédricas tienen un ratio de oxidación mayor que el de los CNTs. No obstante, el proceso de purificación por oxidación también elimina una cantidad apreciable de nanotubos.

\section{Método de la abrasión láser}

El método utiliza una intensa pulsación láser que erosiona un objetivo de carbono con un $0.5 \%$ atómico de níquel y cobalto dentro de un horno tubular calentado a $1200^{\circ} \mathrm{C}$ (figura 1.6). Durante el proceso, se introduce un flujo de gas inerte a través de la cámara de fabricación que arrastra los nanotubos generados hacia el extremo final del horno.

Según [97], por este procedimiento se pueden producir SWNTs de gran calidad (sin defectos estructurales importantes) hasta tamaños de muestra de 1-10 g. Los SWNTs normalmente aparecen agrupados en fajos compactos, con decenas de nanotubos individuales empaquetados en cristales hexagonales debido a la interacción de VDW. Los subproductos y el proceso de purificación utilizados son los mismos que para el método de descarga de arco. 


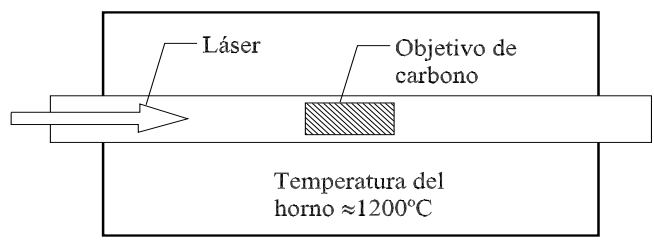

Figura 1.6: Método la abrasión laser (esquema)

\section{Método de deposición química de vapor (CVD)}

El método de fabricación CVD implica el calentamiento de un material catalizador hasta altas temperaturas en un horno tubular y el paso de un hidrocarburo gaseoso a través del tubo reactor durante un periodo determinado de tiempo (figura 1.7). Los materiales depositados sobre el catalizador son recogidos una vez enfriado el sistema a temperatura ambiente.

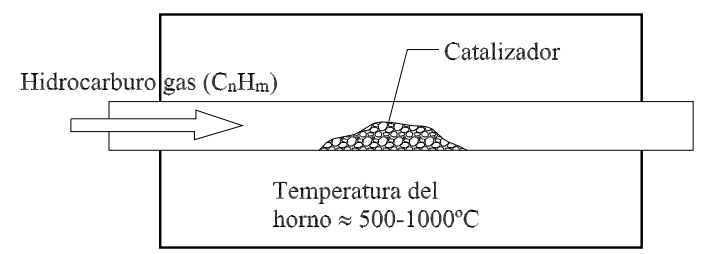

Figura 1.7: Método deposición química de vapor (esquema)

El mecanismo físico-químico de generación de nanotubos en un proceso CVD se produce en las siguientes etapas:

1. Disociación de las moléculas de hidrocarburo $C_{n} H_{m}$ catalizadas por el metal de transición

2. Disolución y saturación de los átomos de carbono en las nanopartículas metálicas

3. La precipitación del $\mathrm{C}$ desde las partículas de metal lleva a la formación de sólidos de C con estructura $s p^{2}$

Los parámetros clave que regulan el proceso de fabricación son: el tipo de hidrocarburo, el tipo de catalizador y la temperatura del horno. Para la fabricación de MWNTs, la mayoría de métodos CVD emplean etileno o acetileno como materia prima, los catalizadores son $\mathrm{Fe}$, $\mathrm{Ni}$ o $\mathrm{Co}$ y la temperatura del horno está sobre los $550-750^{\circ} \mathrm{C}$ (Con altas temperaturas, el $\mathrm{C}$ tiene solubilidades limitadas en estos metales).

El mayor inconveniente en la fabricación de MWNTs mediante CVD es la alta densidad de defectos encontrada en las estructuras, probablemente debido a que la relativamente baja temperatura no proporciona suficiente energía térmica para recocer los nanotubos en estructuras cristalinas perfectas.

Recientemente se ha llevado a cabo la fabricación de SWNTs de reducidas imperfecciones estructurales mediante métodos CVD. Empleando el metano como materia prima, unas temperaturas de $850-1000^{\circ} \mathrm{C}$ y el catalizador adecuado, se pueden obtener rendimientos del $45 \%$ sobre el peso del catalizador.

En general, las propiedades de un buen catalizador serán:

1. Fuertes interacciones con el metal de soporte; las interacciones evitan la agregación de partículas metálicas grandes, que pueden degenerar en MWNTs defectuosos y bajos rendimientos. 
2. Elevadas superficie específica y porosidad; aumentan el rendimiento de fabricación.

3. Mantener las propiedades anteriores a altas temperaturas sin ser pulverizado.

El diámetro de SWNTs sintetizados por el método CVD vendrá condicionado por el tamaño de las nanopartículas catalizadoras. El desarrollo de SWNTs en CVD se producirá por dos vías:

(a) Desarrollo por la base.- Los nanotubos se desarrollan manteniendo un extremo cerrado por una cúpula de fullereno, mientras las partículas catalizadoras permanecen sobre la superficie de soporte. El hidrocarburo se va suministrando desde la base, donde el tubo está anclado al catalizador. Esta vía se presenta en caso de fuertes interacciones entre el catalizador y la superficie de soporte, dando lugar a mayores rendimientos en el desarrollo de SWNTs.

(b) Desarrollo por la punta.- El alargamiento del nanotubo implica elevar una partícula de catalizador desde el soporte y llevarla a su extremo, que permanece abierto durante el proceso de desarrollo. Dicha partícula es la responsable del suministro del carbono necesario. Esta vía se presenta con catalizadores de interacción débil con la superficie de soporte.

\subsubsection{Desarrollo mediante CVD de nanotubos orientados}

Recientemente se han definido estrategias para fabricar SWNTs y MWNTs por CVD, ordenados sobre sustratos dotados de un estampado catalítico. Describimos algunas de ellas a continuación.

\section{Estructuras de MWNTs alineados}

Los MWNTs pueden autoensamblarse mediante interacciones de VDW conforme se generan , quedando como "bosques" orientados [28]. Este mecanismo de autoorientación de MWNTs implica un modo de crecimiento por la base (figura 1.8) suministrando metano como materia prima.

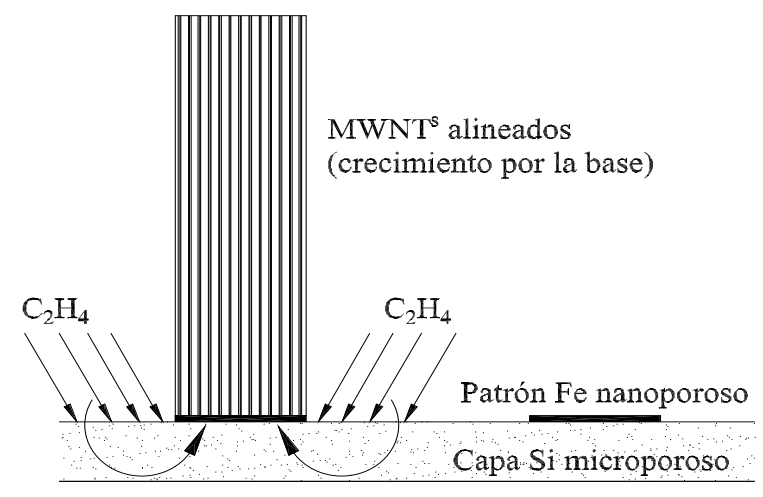

Sustrato de silicio

Figura 1.8: Generación orientada de MWNTs

El silicio poroso es un sustrato ideal para este planteamiento, que se puede obtener por grabado electromecánico de discos de Si en una solución de ácido fluorhídrico en metanol. Sobre este sustrato se genera un patrón catalítico cuadrado evaporando una película de Fe de $5 \mathrm{~nm}$ a través de una máscara superficial con aberturas cuadradas.

Las longitudes de los nanotubos así obtenidos puede regularse en el rango de 10-240 $\mu \mathrm{m}$. 


\section{Desarrollo orientado de SWNTs}

Se pueden obtener estructuras de SWNTs alineados mediante CVD sobre sustratos con patrón catalítico, aunque existen otros métodos para desarrollar redes de SWNTs en direcciones determinadas, suspendidas de pilares de silicio que forman parte del sustrato. El material precursor se transfiere selectivamente a la cabeza de los pilares, llevando a la formación de SWNTs suspendidos de pilar a pilar. En el sustrato base no se forman SWNTs, ya que en él no se dispone ningún material catalizador.

La base de este método consiste en el desarrollo de un precursor en fase líquida (disolución de cloruros de Al, Fe, y Mo en una mezcla de etanol y butanol como solventes), que tiene la ventaja sobre los catalizadores sólidos de permitir la formación de superficies catalíticas uniformes y el estampado catalítico, a gran escala.

Conforme los SWNTs van aumentando su longitud el flujo de metano los mantiene ondulando, porque la velocidad del flujo cerca de la superficie inferior es mucho menor que al nivel de la cabeza de los pilares. Esto evita que los SWNTs queden atrapados en la superficie inferior.

\subsubsection{Aplicaciones de los Natotubos de carbono}

El motivo fundamental por el cual los CNTs han atraído un importante esfuerzo por parte de investigadores y científicos reside en las extraordinarias propiedades resistentes y de conductividad eléctrica que poseen. Dichas propiedades ya se han explotado en ciertas aplicaciones interesantes en la actualidad, aunque resta materializar otros prometedores usos de los CNTs en un futuro próximo. Describimos a continuación una buena parte de las aplicaciones de los CNTs, con la intención de dar una idea de su importancia en el marco científico-tecnológico.

\section{Composites reforzados}

Las fibras de carbono convencionales se han empleado en la fabricación de composites ligeros, de alta resistencia y altas prestaciones, tanto en elementos estructurales para aviación como para aeronaves espaciales. Por tanto, cabe esperar que los CNTs sean los candidatos perfectos para aplicaciones estructurales. De hecho, la NASA ha invertido recientemente grandes cantidades de dinero en desarrollar composites basados en nanotubos para aplicaciones tales como la misión de Marte.

Los trabajos teóricos preliminares y algunos experimentos recientes sobre nanotubos individuales (sobre todo MWNTs) han confirmado que los nanotubos son una de las estructuras más rígidas jamás descubiertas [99], [112], [115]. Dado que los enlaces covalentes C-C son uno de los más resistentes en la naturaleza, una estructura basada en una disposición perfecta de estos enlaces orientados a lo largo del eje del nanotubo produciría un material extremadamente resistente. Algunos estudios teóricos sugieren que los SWNTs podrían tener un módulo de Young longitudinal de $1 \mathrm{TPa}$ [115], valor similar al de la lámina de grafeno libre de defectos. Sin embargo, para MWNTs la resistencia real en situaciones prácticas viene afectada por el deslizamiento de alguna de las láminas cilíndricas constituyentes respecto al resto (modo de fallo espada-vaina) [119] seguida de la rotura de cada cilindro individual. La resistencia a tracción observada para MWNTs individuales corresponde a $\sigma_{t}<60$ GPa. Se han llevado a cabo también experimentos de grupos de SWNTs a tracción [119] y a flexión [93], viéndose condicionada la resistencia final por el deslizamiento entre SWNTs individuales.

Aunque el ensayo de SWNTs es complicado y requiere dispositivos de carga a escala nano, algunos experimentos han proporcionado ideas valiosas sobre su comportamiento mecánico. Por ejemplo, en uno de los experimentos iniciales [99], se asumió que nanotubos salientes de los huecos de una rejilla de muestra en un microscopio TEM son equivalentes a ménsulas homogéneas. La amplitud de vibración en los extremos de los tubos fueron medidas a partir del desenfoque de las puntas de los nanotubos y relacionadas con el módulo de Young. 
Las resistencias teóricas a tracción para SWNTs individuales están en torno a 300 GPa, pero los mejores valores experimentales son próximos a $52 \mathrm{GPa}$ [118]. El mecanismo de deformación y fractura de SWNTs es sorprendente, ya que pueden mantener grandes deformaciones (40\%) en tracción sin mostrar signos de fractura [115]. La reversibilidad de las deformaciones, incluso las asociadas a problemas geométricamente no lineales como la abolladura o el pandeo, ha sido observada directamente en imágenes de TEM [27].

El comportamiento plástico de los nanotubos a tracción se asocia a la formación de defectos de Stone-Wales (S-W), consistentes en una rotación de $90^{\circ}$ de un enlace covalente, formándose dos pentágonos y dos heptágonos adyacentes. Al aumentar la deformación, la sucesiva rotación de enlaces va desplazando relativamente los dos pares 5-7 hasta la fractura final [7], [123], [124]. Esta separación de defectos crea un estrechamiento local y un cambio de helicidad del entramado en la zona implicada del CNT, lo que permite intuir aplicaciones a nuevos dispositivos que reaccionen al esfuerzo mecánico modificando sus características electrónicas. En general, resulta más fácil formar defectos $\mathrm{S}-\mathrm{W}$ en los tubos de radio menor [123], aunque los tubos mayores suelen tener mayor número de defectos a la misma deformación relativa, siendo la densidad superficial de defectos constante. Zhao [124] afirma que los defectos S-W no son termodinámicamente favorables hasta deformaciones del $10 \%$ en CNTs ZigZag, cuya quiralidad deberá permitir tensiones de tracción elevadas.

Incluso Ariza et al. [3], [4], [5] analizan defectos similares a los S-W en la lámina de grafeno aislada y concluyen que las geometrías encontradas aplicando su propia teoría de la dislocación (basada en técnicas MD) son estables hasta temperaturas de $2500^{\circ} \mathrm{K}$, aunque experimentan cierta relajación en el curso de la Dinámica Molecular. Esta idea permite intuir que los defectos estructurales en SWNTs también son dinámicamente estables para un amplio rango de temperaturas.

A pesar de que los compuestos poliméricos reforzados con nanotubos constituyen un área de aplicación de CNTs prometedora, no han habido muchos experimentos concluyentes que demuestren las ventajas de usar nanotubos como refuerzo en lugar de las fibras de carbono tradicionales. El problema fundamental es crear una interfaz entre los nanotubos y la matriz polimérica que permita una buena transferencia de carga matriz-nanotubo. Esto se debe sobre todo a que: (i) los nanotubos son lisos atómicamente y tienen prácticamente los mismos diámetros y esbelteces que las cadenas de polímeros, y (ii) los nanotubos suelen estar organizados en agregados que se comportan de forma diferente respecto a los CNTs individuales. La facilidad de deslizamiento entre capas individuales de MWNTs y entre tubos individuales en grupos de SWNTs podrían ser factores limitantes en la transferencia de carga.

Si se desea aumentar las ventajas de los nanotubos como elementos de refuerzo en composites de alta resistencia, los agregados necesitan ser dispersados o agrupados sin orientación predominante, para impedir el deslizamiento [1], aunque actualmente esta tarea sigue siendo un reto. Además, las superficies de los CNTs deben ser activadas químicamente para conseguir interfaces resistentes con las cadenas de polímeros envolventes.

En general, el refuerzo con CNTs aumentará la ductilidad de los composites absorbiendo energía durante su prolongada fase elástica. Esto es especialmente importante para composites de matriz cerámica. Incluso bajo cargas de compresión los CNTs ofrecen mejores características que las fibras de carbono, debido a su flexibilidad y baja propensión a la fractura en estos casos. Por otra parte, la baja densidad de los CNTs es una clara ventaja en comparación con los composites reforzados (aleatoriamente) con fibras de carbono.

Otras aplicaciones interesantes de los polímeros reforzados con CNTs serán los adhesivos, donde una distribución de nanotubos sobre una película de polímero puede alterar las características de las cadenas poliméricas debido a las interacciones con los CNTs.

Dentro del campo de los composites poliméricos reforzados, podemos encontrar otras aplicaciones no estructurales de los nanotubos. En los polímeros luminescentes PPV (Poli PhenyleneVinylene), los CNTs han demostrado producir grandes aumentos en la conductividad eléctrica (cerca de ocho órdenes de magnitud) comparado con el polímero inicial, con muy poca pérdida de fotolumi- 
nescencia o electroluminescencia. Además, el composite es bastante más resistente mecánicamente que el polímero puro y presenta mayor capacidad de impedir el colapso o desintegración por calor, ya que los CNTs actúan como sumideros térmicos.

\section{Aplicaciones potenciales de los CNTs en microelectrónica}

Los CNTs están dotados de la combinación adecuada de propiedades (diámetro nanométrico, integridad estructural, alta conductividad eléctrica y estabilidad química) que los convierte en aventajados emisores de electrones frente a los emisores convencionales, ya que muestran un menor umbral de campo eléctrico (necesario para la emisión) y unas densidades de corriente superiores a los emisores convencionales. Los emisores de electrones han sido investigados extensivamente para aplicaciones tecnológicas, tales como pantallas planas, fuentes emisoras en microscopios de electrones y amplificadores de microondas.

En la actualidad, los dispositivos prototipo de emisión de electrones basados en CNTs se pueden clasificar en:

(a) Elementos emisión de rayos catódicos: han sido fabricados con materiales basados en CNTs como emisores de campo.

(b) Pantallas planas: los CNTs se emplean como fuente de emisión de electrones.

(c) Tubos de descarga por gas en redes de telecomunicaciones: los electrodos del dispositivo se protegen mediante CNTs. Se trata de electrodos de molibdeno con varios materiales entre capas cubiertos de SWNTs, útiles por ejemplo en redes de líneas ADSL (asymmetric digital signal line).

\section{Aplicaciones en almacenamiento de energía}

Durante décadas se han empleado materiales carbonosos y electrodos de fibras de carbono en pilas de combustible, baterías y muchas otras aplicaciones electromecánicas. Varios experimentos señalan que la cinética de transferencia de electrones tiene lugar más rápidamente en los CNTs que en los electrodos de carbono convencionales.

Se han utilizado MWNTs puros y MWNTs depositados con catalizadores metálicos (Pd, Pt, Ag) para catalizar eléctricamente una reacción de reducción de oxígeno, la cual es importante en baterías de combustible. Por otra parte, las propiedades de las nanofibras de carbono (que básicamente son nanotubos defectuosos) generadas catalíticamente son deseables para condensadores electromecánicos de alta potencia.

Describimos a continuación dos aplicaciones fundamentales de los CNTs como instrumentos de mejora en dispositivos de almacenamiento de energía:

(a) Baterías recargables de litio.- El mecanismo básico de trabajo de estas baterías es la intercalación química de Litio entre dos electrodos conectados. Las actuales baterías de Li usan óxidos de metales de transición como cátodos y materiales carbonosos como ánodos. Siempre es deseable obtener baterías con alta capacidad de almacenamiento, corto periodo de carga y un prolongado ciclo temporal.

Se especula que puede obtenerse una capacidad más alta de inclusión de Li en CNTs si todos los sitios intersticiales (espacios entre láminas, canales entre tubos y núcleos huecos) son accesibles para la intercalación. La elevada capacidad y el alto rendimiento conseguidos hasta ahora justifican estudios posteriores del potencial de utilización de los CNTs como electrodos en baterías. 
(b) Almacenamiento de hidrógeno.- Los materiales con altas capacidades de almacenamiento de Hidrógeno son deseables para aplicaciones de almacenamiento de energía. Debido a su geometría cilíndrica hueca y diámetros a escala nanométrica, se prevé que los CNTs sean capaces de almacenar líquidos y gases en los núcleos interiores por capilaridad. Por tanto, se perfilan como candidatos al almacenamiento de Hidrógeno.

Si todas las moléculas de $\mathrm{H}$ se suponen en el interior de los nanotubos, se obtendría una densidad de empaquetado de moléculas $H_{2}$ dentro de los tubos muy superior a la esperada a partir de la distancia usual $\mathrm{H}_{2}-\mathrm{H}_{2}$. La absorción de $\mathrm{H}_{2}$ por parte de SWNTs se ve incrementada en un $5 \%$ en peso al abrir sus extremos mediante un proceso de oxidación, y en un $8 \%$ aumentando la presión de gas hasta $100 \mathrm{~atm}\left(\mathrm{~T}=80^{\circ} \mathrm{K}\right)$, lo cual sugiere que los nanotubos poseen la mayor capacidad de almacenamiento de $\mathrm{H}$ de todos los materiales carbonosos.

En este momento, todavía no ha quedado completamente determinado si los nanotubos tendrán aplicaciones tecnológicas reales en el área del almacenamiento de Hidrógeno. Los valores aportados por la literatura deberán ser verificados sobre materiales bien caracterizados bajo condiciones controladas. De hecho, aún persiste una falta de entendimiento detallado del mecanismo de almacenamiento y del efecto del tratamiento de materiales sobre el almacenamiento de $\mathrm{H}$.

\section{Nano-sondas y sensores}

Teniendo en cuenta su tamaño extremadamente pequeño, elevada conductividad eléctrica, elevada resistencia mecánica y gran flexibilidad frente a la flexión, los nanotubos pueden convertirse en indispensables como nano-sondas. Las aplicaciones de estas sondas podrían ser:

1. Sensores para monitorización estructural [48].

2. Dispersión de medicamentos [57] transportando partículas en su interior. Por ejemplo, para suministro de anticancerígenos.

3. Captado de imágenes de alta resolución en microscopios.

4. Emisores de campo.

5. Nanoelectrodos.

6. Nanolitografía.

La ventaja de emplear puntas de nanotubos en un microscopio de fuerza atómica (AFM) es su esbeltez y la posibilidad de captar detalles en las imágenes (tales como pequeñas roturas superficiales), que son casi imposibles de detectar mediante las puntas de silicio o metálicas de mayor tamaño. Incluso permiten capturar imágenes de moléculas biológicas como el ADN. Adicionalmente, debido a la gran elasticidad de los nanotubos, las puntas no sufren roturas al entrar en contacto con los sustratos.

Otra aplicación de los nanotubos en AFM consiste en su uso como herramientas activas para la manipulación superficial (nano-manipuladores). Se ha mostrado que si un par de CNTs puede ser dispuesto apropiadamente en una punta de AFM, pueden ser controlados como unas pinzas para sujetar y/o liberar nanoestructuras dispuestas sobre superficies.

Dado el acoplamiento entre la conductividad eléctrica y la deformación mecánica [83], se han fabricado actuadores nano-electromecánicos (NEMS) mediante láminas de SWNTs. Se ha mostrado que pequeños voltajes (de unos pocos Voltios) aplicados a cintas de nanotubos laminados (con un polímero) suspendidas de un electrodo, flectan la lámina hasta grandes deformaciones, imitando el mecanismo de activación presente en los músculos naturales. Este interesante comportamiento 
sugiere aplicaciones en biomecánica (fabricación de músculos artificicales) o en medicina como catéteres médicos.

Se ha mostrado también que los nanotubos pueden usarse como avanzados sensores químicos en miniatura. Las resistividades eléctricas de los SWNTs varían sensiblemente con la exposición a ambientes gaseosos conteniendo moléculas de $\mathrm{NO}_{2}, \mathrm{NH}_{3}$ y $\mathrm{O}_{2}$. Monitorizando el cambio en la conductancia de los nanotubos se puede detectar la presencia de estos gases.

\section{Moldes}

Puesto que los nanotubos tienen canales relativamente rectos y estrechos en sus núcleos, se especuló inicialmente con la posibilidad de rellenar estas cavidades con materiales externos para fabricar nanocables. Las fuerzas capilares existentes en los CNTs serían las responsables de mantener en su interior líquidos o gases. Actualmente, los nanotubos se han empleado como moldes para crear nanocables de diferentes composiciones y estructuras. Su topología cerrada debe abrirse previamente al rellenado mediante un sencillo proceso químico de oxidación.

Es posible también fabricar nanoestrucuras basadas en CNTs envolviendo uniformemente los nanotubos con estructuras moleculares orgánicas o inorgánicas. Se espera que estos composites tengan interesantes propiedades mecánicas y eléctricas debido a la combinación de efectos dimensionales y propiedades de interfaz. Esta interfaz entre el nanotubo y el compuesto envolvente (óxidos depositados a capas) es atómicamente plana debido a la ausencia de enlaces covalentes atravesando la superficie. Se ha demostrado que los nanotubos pueden ser eliminados por oxidación posteriormente al recubrimiento, dejando nanotubos independientes constituidos por óxidos, con espesor de pared nanométrico. Estos nuevos tubos cerámicos podrían tener aplicaciones interesantes en catálisis.

\section{Retos en las aplicaciones de los CNTs}

En las secciones anteriores se han descrito una gran parte de las aplicaciones de los CNTs estudiadas hasta la fecha. Sin embargo, quedan muchos retos por vencer en el desarrollo de los nanotubos en dispositivos y estructuras funcionales.

En primer lugar, el mecanismo de formación de los nanotubos, aunque semejante al de los fullerenos, sigue siendo un fenómeno poco conocido. Por tanto, no es posible todavía desarrollar estas estructuras de una forma controlada (en cuanto a sus detalles estructurales). Han habido algunos éxitos en desarrollar nanotubos de diámetro predefinido (y en menor medida con una quiralidad predeterminada) ajustando las condiciones de fabricación por prueba y error. Especialmente en aplicaciones electrónicas, que dependen directamente de la estructura electrónica de los nanotubos, esta incapacidad en la selección previa del diámetro y la quiralidad representa un inconveniente.

Muchas predicciones de la aplicabilidad en dispositivos están basadas en la unión de nanotubos mediante la incorporación topológica de defectos en sus entramados. Hasta el momento, no hay forma controlable de producir conexiones entre nanotubos. Sin embargo, algunos trabajos recientes sugieren la posibilidad de construir estas estructuras interconectadas por irradiación de electrones y por medio de patrones o moldes [26, cap. 14].

Para aplicaciones materiales tales como el refuerzo de composites, donde la helicidad tiene una influencia mucho menor, las cantidades de nanotubos que pueden ser manufacturadas son demasiado pequeñas en comparación con las que la industria podría necesitar. Hoy por hoy, no existen técnicas disponibles de producción de CNTs con calidad y pureza razonables en la escala del Kg, siendo la necesidad industrial del orden de Toneladas para estas aplicaciones. Esto hace que el precio de mercado sea actualmente demasiado alto (200\$/ $g$ en USA).

La revolución que se está produciendo en el campo de la nanotecnología requiere manipular estructuras a escala atómica. Esto se ha conseguido empujando nanotubos situados en un sustrato 
mediante la punta de un AFM, aunque dicha manipulación persiste como reto fundamental en este campo, junto con muchos otros.

\subsection{Motivación}

Uno de los dos puntos de partida de esta tesis se sitúa en la tesis de Lázaro [56]. En ella se desarrolla la formulación material y espacial del modelo de Reissner-Simó, así como su conexión con la teoría no lineal de la Elasticidad. El análisis de Lázaro pone de manifiesto la conexión formal entre las ecuaciones de la estática de piezas alargadas y las ecuaciones de la dinámica de sólidos rígidos (analogía dinámica), actualizando la analogía cinética de Kirchhoff . Finalmente, desarrolla el elemento finito de Simó y Vu-Quoc y un nuevo elemento finito empleando la forma material del operador tangente, contrastando la precisión numérica de ambos elementos mediante diversos ejemplos.

El otro punto de partida tiene lugar en los trabajos de Chandraseker y Mukherjee [13], y de Krylov et. al. [51], ambos presentados en el $6^{\circ}$ Congreso Internacional de la IASS-IACM. En ambos se trata de modelos viga aplicados a estructuras de escala micro o nano. En particular, en [13] se especula con la aplicabilidad de un modelo de piezas alargadas tipo Cosserat al análisis estructural de los nanotubos de carbono monocapa.

La idea inicial de la tesis consistió en aplicar un modelo de piezas alargadas tipo Reissner-Simó al análisis resistente de los nanotubos de carbono, introduciendo si fuera necesario las modificaciones pertinentes para validarlo experimentalmente. El motivo fundamental reside en que estos nanoelementos son altamente flexibles (presentan una gran esbeltez), proporcionando un caso real único para la aplicación de los modelos de piezas alargadas, sólo presente en algunas situaciones singulares dentro del ámbito de la Ingeniería Estructural.

Al considerar con mayor detalle las características estructurales de los nanotubos de carbono, se ponen de manifiesto algunas modificaciones y/o extensiones del modelo de piezas alargadas tipo Reissner-Simó. Incluso en algunos casos se pone en entredicho la aplicabilidad del propio modelo, como se describe a continuación:

(a) La configuración de cilindro hueco con una elevada deformabilidad transversal, invalida la hipótesis de sección transversal indeformable de la pieza de Cosserat más allá de la linealidad geométrica. La extensión natural del modelo consiste en considerar la sección transversal como una curva deformable en su propio plano, tal como desarrollan Gould y Burton [31].

(b) La orientación o quiralidad de las celdas hexagonales que forman cada lámina de nanotubo introduce una nueva dificultad, consistente en la consideración de la simetría helicoidal en el modelo, como un caso particular de simetría transversal. Healey [36] plantea la introducción de estas simetrías orientadas al estudio de moléculas de ADN por su quiralidad, presente también en los nanotubos de carbono. Un desarrollo algo más abstracto de la simetría material de piezas alargadas puede consultarse en [72].

(c) La propia constitución de la lámina de grafeno como una malla de celdas hexagonales, donde cada átomo de $\mathrm{C}$ está situado en un vértice de la malla y cada arista representa un enlace covalente $\mathrm{C}-\mathrm{C}$, cuestiona la adecuación de un modelo continuo a la respuesta estructural de los nanotubos, sobre todo si consideramos un grupo de átomos de $\mathrm{C}$ no demasiado numeroso, donde el promedio de magnitudes físicas es cuestionable. Algunos autores [32], [34] parametrizan la aplicabilidad del continuo en función de las dimensiones del tubo.

Dadas estas particularidades, decidimos centrar la investigación en el análisis estructural de los nanotubos de carbono adaptando el modelo a la reproducción de su respuesta mecánica a nivel molecular. De todos los modelos desarrollados hasta la actualidad, descritos con mayor detalle en el 
Capítulo 2, optamos por un modelo basado en la Mecánica Molecular y formado por un sistema de barras y muelles que reproduce la geometría de malla hexagonal de cada lámina de grafeno constituyente del nanotubo. Dicho modelo se sitúa en un punto intermedio entre los costosos cálculos (en tiempo y esfuerzo computacional) de Dinámica Molecular y los modelos continuos, incapaces de reproducir los detalles estructurales (salvo si se introducen algunas modificaciones que llevan a una formulación compleja) tales como la quiralidad o las imperfecciones de la malla.

\subsection{Objetivos de la tesis}

En la presente Tesis, se persigue analizar la respuesta estructural de los nanotubos de carbono monocapa mediante un modelo de mecánica estructural molecular (MSM) formulado a partir del principio de los trabajos virtuales. Con esta finalidad, planteamos los siguientes objetivos, no tratados (o poco desarrollados) en este tipo de modelos hasta el momento:

1. Estudiar la determinación estática y cinemática del modelo, para descartar posibles modos internos de mecanismo y determinar modos de autotensión. En caso de existir algún modo de mecanismo, cabría identificar las condiciones necesarias para su formación y las condiciones de contorno que producirían la estabilidad estructural, estableciendo así los límites de aplicabilidad del modelo.

2. Construir una formulación explícita de las ecuaciones que gobiernan la respuesta de un sistema de barras articuladas con resortes angulares en los nudos (modelo MSM 'stick-spiral') mediante un desarrollo energético inspirado en una formulación clásica en rigidez [69]. Se trata de definir una conexión conceptual entre las diferentes interacciones atómicas dentro del nanotubo y los elementos estructurales de nuestro modelo para obtener una formulación compacta independiente del estado de cargas externas. Esta idea constituye una de las novedades fundamentales de la investigación, ya que hasta la fecha las referencias que tratan este modelo desarrollan formulaciones parciales limitadas a casos particulares de carga [14], [15], [77], [78], [106], [114].

3. Si entendemos la predeformación o preenergía como el conjunto de deformaciones y tensiones iniciales que se introducirán en el CNT por efecto de la curvatura cilíndrica respecto de la configuración de equilibrio de la lámina plana de grafeno, buscaremos analizar su influencia en la respuesta estructural de los nanotubos de carbono monocapa. En este sentido, la predeformación ha sido tratada casi exclusivamente en el marco de la dinámica molecular (MD) (por ejemplo, ver [43], [94], [115]), siendo una innovación dentro del modelo MSM donde suele ser ignorada.

4. Parametrizar la respuesta estructural del modelo frente a los estados de tensiones habituales (tracción, flexión, torsión, etc) en función de dimensiones estructurales y geométricas, tales como la quiralidad, el diámetro o la esbeltez del conjunto.

5. Verificar la importancia en el ajuste de los parámetros constitutivos del enlace covalente C-C en las relaciones constitutivas de los nanotubos como conjunto. Cabe estudiar si asumir una relación constitutiva lineal del enlace (y de los resortes) es suficientemente aproximado para reproducir los resultados experimentales correctamente o si por el contrario se requiere adoptar potenciales interatómicos más sofisticados.

6. Dentro del marco de la no linealidad geométrica, estudiar los factores de cargas críticos bajo los patrones de carga tipo (compresión, flexión, torsión), así como desarrollar un algoritmo general que permita el trazado de trayectorias no lineales de equilibrio y el estudio de sus 
características. Asimismo, se persigue verificar si el modelo reproduce la no linealidad geométrica previamente a la no linealidad mecánica, como se ha deducido experimentalmente [27] y analizado teóricamente mediante técnicas MD [43], [94], [110].

7. Disponer de un elemento de contraste respecto a los datos experimentales existentes o bien respecto a otros modelos teóricos desarrollados hasta el momento, así como establecer un punto de partida para futuras extensiones del modelo, ya sea del tipo 'stick-spiral' o dentro del marco del continuo.

\subsection{Contenido}

Esta tesis doctoral consta de siete capítulos organizados como sigue:

En el capítulo1 se proporciona una breve descripción del problema, así como del modelo objeto de la investigación. Asimismo, se explican los motivos que derivaron en el empleo de dicho modelo y los objetivos a cubrir en este estudio.

El capítulo 2 contiene una revisión del estado del Arte acerca de los modelos y métodos empleados en el análisis mecánico de nanotubos de carbono, donde se describen brevemente los conceptos fundamentales de cada uno, sus ventajas e inconvenientes. Se proporciona también una clasificación general de los modelos empleados y la situación del presente modelo con el objetivo de enmarcarlo dentro de la panorámica general de análisis desarrollada hasta este momento.

En el capítulo 3 se analiza la estabilidad estática y cinemática del modelo empleando la descomposición en valores singulares (SVD) de la matriz de equilibrio. El objetivo de ese capítulo consiste en estudiar si la estructura es autoestable o aparecen modos de mecanismo linealmente independientes que limiten su estudio mediante un análisis estático clásico. En caso de ser así, debemos analizar las condiciones de contorno necesarias para su determinación cinemática y establecer las limitaciones del modelo en cuanto a sus restricciones de apoyo se refiere.

El capítulo 4 está dedicado al análisis geométricamente lineal del modelo 'stick-spiral' mediante un procedimiento energético por trabajos virtuales que establece una conexión entre los distintos tipos de interacción atómica (longitudinal y angular) y cada uno de los elementos estructurales del modelo. Por este procedimiento es posible desarrollar la expresión simbólica de la matriz de rigidez de cada elemento, así como aprovechar las ventajas del ensamblaje booleano, usual en el análisis matricial de estructuras. Con el objetivo de hacer un primer contraste en cuanto a la no linealidad constitutiva se introducen dos potenciales interatómicos (siendo uno de ellos no lineal) y se comparan los resultados obtenidos con cada uno. Asimismo, se desarrollan cálculos con y sin predeformación para analizar su influencia en la respuesta mecánica de SWNTs. En general, este capítulo proporciona la base de la formulación general en rigidez para el modelo MSM además de permitir un primer contraste de valores con otros autores.

En el capítulo 5 se estudia la extensión del modelo a problemas geométricamente no lineales, desarrollando algoritmos específicos para el trazado de trayectorias de equilibrio no lineales y el cálculo de deformaciones y cargas críticas. Se obtienen expresiones simbólicas para las matrices de rigidez tangente de cada elemento mediante la ecuación de trabajos virtuales, además de desarrollar los métodos de continuación necesarios para el análisis del comportamiento frente a la inestabilidad de nanotubos monocapa bajo compresión, flexión y torsión. En este contexto, la generalidad de la formulación permite introducir fácilmente distintos potenciales interatómicos sin modificar las ecuaciones gobernantes, siempre que las interacciones frente a las distintas deformaciones entre átomos aparezcan desacopladas en la función potencial. Desarrollada la formulación, se obtiene una batería de resultados bajo los estados de carga habituales en análisis de estructuras (compresión, flexión y torsión) y se analizan en comparación a otros referencias publicadas que emplean modelos atomísti$\cos$. 
El capítulo 6 critica los posibles estados de predeformación y propone una nueva forma de encontrar otras geometrías iniciales de equilibrio (diferentes a la definida por el mapeo conforme) mediante una minimización energética del potencial previa a la actuación de cualquier carga externa. Este capítulo se puede entender como una posible mejora numérica a la introducción del pretensado, ya que si somos capaces de aproximar con mayor precisión la configuración inicial de equilibrio, la influencia de la curvatura del cilindro en la respuesta mecánica se verá modificada considerablemente.

En el capítulo 7 se recogen las conclusiones, se incluyen las aportaciones originales de la tesis y se proponen nuevas líneas de investigación.

Finalmente, en el Apéndice A se proporciona un índice de acrónimos frecuentes en la literatura, así como la notación de las variables fundamentales empleadas en la formulación para facilitar una consulta más directa. 


\section{Capítulo 2}

\section{Estado del Arte}

\subsection{Clasificación esquemática de los métodos de análisis para CNTs}

Desde el descubrimiento de los CNTs en 1991 [42] hasta la actualidad, una parte importante de la investigación relacionada con estas singulares moléculas se ha destinado a la predicción de su respuesta mecánica frente a acciones o estímulos externos. En algunos casos se han adoptado modelos ya existentes para modelar el comportamiento resistente de los CNTs, mientras que en otros se han definido nuevos modelos con características propias. En cualquier caso, no existe consenso respecto a la idoneidad de un único modelo capaz de reproducir la respuesta estructural frente a un conjunto de acciones general. Sin embargo, la mayoría de los modelos planteados hasta la fecha son capaces de describir (aunque en algunos casos sólo cualitativamente) algunas propiedades de los CNTs presentadas en el Capítulo 1

A pesar de las dificultades físicas (consecuencia de su reducido tamaño) en la manipulación y obtención de imágenes de CNTs, la primera aproximación al problema fue experimental: [43] con high resolution electron microscope(HREM), [99] con transmission electron microscope(TEM) y [112] con atomic force microscope(AFM) 1. Dada la configuración de pieza alargada que puede deducirse a partir de las imágenes de los microscopios electrónicos, el tratamiento de datos incluido en estos estudios experimentales se ha llevado a cabo tradicionalmente mediante un sencillo modelo viga de Navier sin consideraciones adicionales. No obstante, pronto aparecen nuevos comportamientos como la respuesta no lineal a flexión o compresión (supuestamente originada por un problema de inestabilidad de la lámina grafítica o rippling mode) que cuestionan la hipótesis de sección transversal indeformable asociado a los modelos de piezas alargadas.

Teniendo en cuenta el relativamente reducido número de átomos de C implicados en un SWNT, la homogeneización de las características mecánicas de estos elementos a un medio continuo es más que dudosa, poniéndose de manifiesto en la dificultad de asignación unívoca de un espesor de lámina equivalente y un módulo de Young al modelo. En consecuencia, una gran parte de autores ([89], [43] y [115] entre otros) optan por modelos de Dinámica Molecular (MD), consistentes en la interacción mutua de un sistema de puntos que representan los átomos de C. Estos sofisticados modelos son muy fiables, aunque a costa de un elevado coste computacional (hasta la fecha, los sistemas tratados pueden llegar a contener del orden de $10^{6}$ átomos de C [64]).

${ }^{1}$ Los acrónimos pueden consultarse en el Apéndice A 


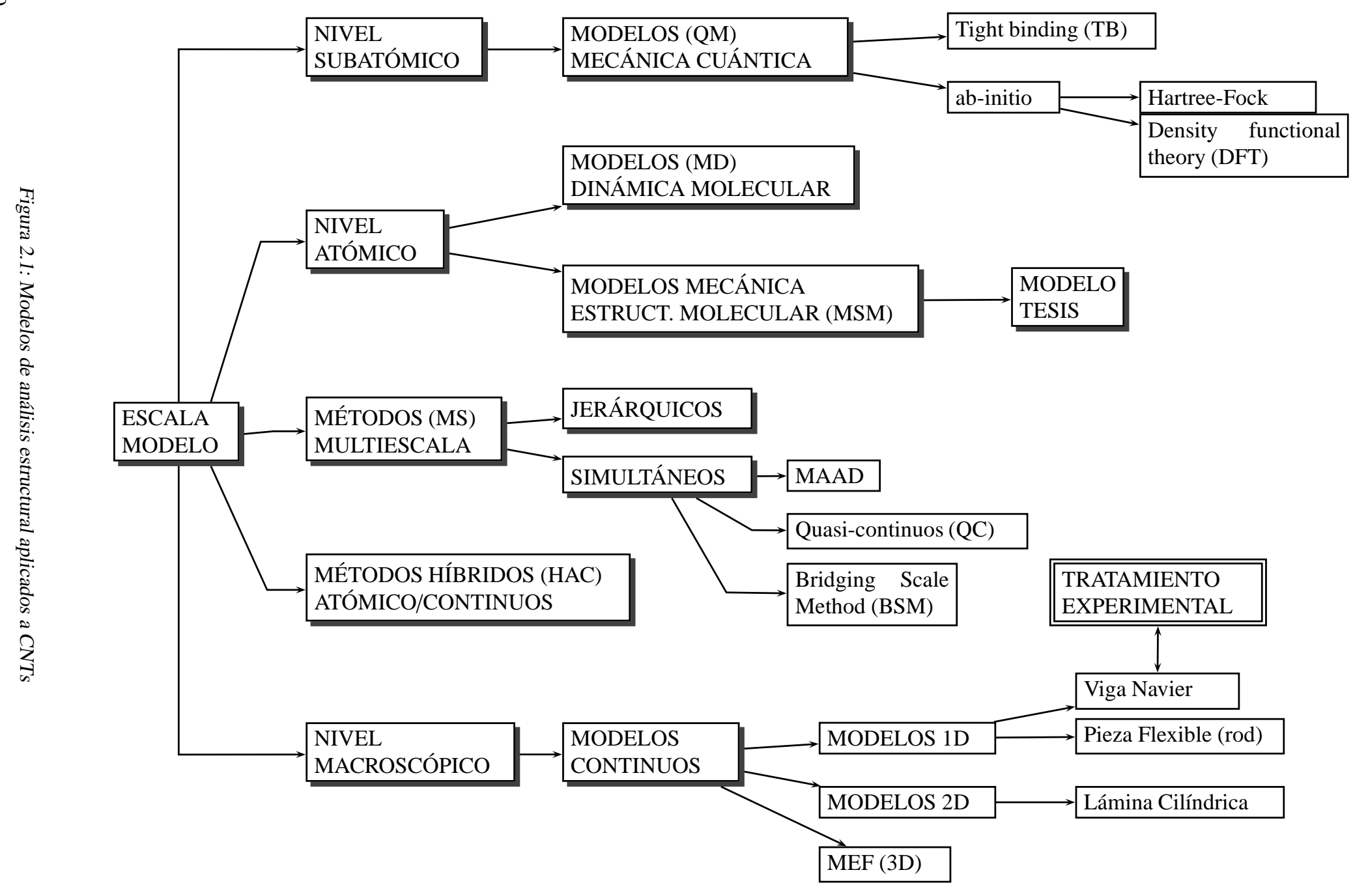


Actualmente, la tendencia en la modelización de CNTs consiste en la búsqueda de nuevos modelos que reproduzcan fielmente sus características resistentes a un coste computacional aceptable. No es de extrañar la aparición de modelos que tratan el problema a una escala intermedia (entre el nivel atómico y el continuo), validados normalmente mediante técnicas MD.

Esta situación hace patente la dificultad de clasificación general de modelos y métodos existentes, debido a que los límites conceptuales entre ellos aparecen desdibujados en la literatura. A pesar de ello, hemos trazado un esquema de conjunto en la figura 2.1 a partir de las directrices resumidas en [67], con el objetivo de situar el modelo de la tesis en el marco general de estudios realizados.

El criterio inicial de clasificación está relacionado con la escala o dimensión del elemento representativo. En una segunda etapa los grupos se han subdividido en función del método de análisis, la configuración geométrica del modelo, o bien el tipo de relación entre sus elementos. Cabe señalar que el tratamiento experimental no es un modelo en si, aunque dado su carácter excepcional como técnica de aproximación, se ha incluido en un grupo individual.

Tomando como referencia el esquema representado en la figura 2.1 describiremos a continuación los modelos desarrollados hasta la actualidad, y discutiremos sus ventajas e inconvenientes.

\subsection{Modelos de mecánica cuántica (QM)}

Los modelos que emplean técnicas de cálculo QM consisten en un sistema de puntos materiales (o de esferas sólidas sin estructura interna) que ejercen fuerzas derivadas de potencial sobre su vecindad. Habitualmente se consideran los núcleos atómicos y los electrones como elementos básicos del sistema (nivel subatómico) sin intercambio de energía entre ellos. Sin embargo, cada átomo dentro del sistema representa un complejo dominio físico que puede evolucionar en el tiempo e intercambiar energía con el entorno.

La dependencia de la función potencial empleada en MD (sección 2.4.1) respecto a la separación entre átomos y moléculas, y su orientación mutua puede en principio ser obtenida a partir de cálculos QM; lo cual proporciona una conexión energética entre las escalas atómica y subatómica.

El estado energético de un núcleo atómico aislado se puede determinar resolviendo la ecuación de onda estacionaria de Schrödinger. La función de onda de Schrödinger $\psi$ (conocida como orbital atómico), solución de dicho sistema de ecuaciones en derivadas parciales, proporciona la función de densidad de probabilidad de las posiciones de los electrones y sirve exclusivamente como una herramienta matemática, ya que no se puede determinar experimentalmente. La integración de esta función de densidad de probabilidad en un dominio espacial que envuelve al núcleo atómico, resulta en la probabilidad de encontrar un electron en ese dominio espacial.

La obtención analítica de la función $\psi$ resulta compleja, incluso para los casos más sencillos como una molécula de $\mathrm{H}_{2}$, con lo que se han desarrollado una variedad de métodos numéricos para obtener funciones de onda aproximadas en sistemas un elevado $\mathrm{n}^{\mathrm{O}}$ de átomos y de electrones, tales como los métodos tight binding y ab initio (o first principles).

\subsubsection{Método tight binding (TB)}

El objetivo de este método es construir la función de ondas aproximada de un único electrón interactuando con dos o más núcleos fuente. Esta función, conocida como orbital molecular, es empleada posteriormente en la obtención de funciones de prueba en los métodos Hartree-Fock. En general, se supone que los orbitales moleculares se pueden obtener como combinación lineal de los orbitales atómicos individuales correspondientes a cada núcleo atómico.

De acuerdo con el principio variacional de la mecánica cuántica, la energía de un orbital molecular elegido arbitrariamente es mayor o igual que la energía del orbital real. Por tanto podemos 
obtener los coeficientes de combinación de orbitales a partir de la minimización de la energía total del sistema.

Este sofisticado método ha sido aplicado con éxito al estudio de la inestabilidad de la lámina de grafeno (rippling mode y/o abolladura). Por ejemplo, [37] trata el comportamiento a compresión de SWNTs mediante TB, [90] somete SWNTs a flexión y torsión hasta la abolladura para analizar la influencia de las deformaciones sobre sus propiedades eléctricas y de resistencia, y [94] muestra la recuperación de las deformaciones post-críticas elásticamente.

Por otra parte, [123] aplica el método TB al estudio de la formación de defectos de Stone-Wales (5-7-7-5) en SWNTs, y [124] lo emplea en la determinación de las barreras energéticas de activación y formación de tales defectos.

\subsubsection{Métodos ab initio}

Dentro de los métodos conocidos en las referencias como ab initio o first principles encontramos los métodos tipo Hartree-Fock y la Teoría del funcional de densidad (DFT). Algunos autores [37], [94], [124] emplean los métodos ab initio como elemento de comparación respecto al método TB, con las simulaciones mencionadas en la sección 2.2.1 En otros casos (por ejemplo [89]) se emplean estos métodos para simular SWNTs a tracción y compresión, incluso [103] valida el modelo lámina mediante ab initio y MD simulando SWNTs a tracción y torsión.

Otros ejemplos de aplicación de estos métodos menos relacionados con esta tesis, son la tracción de nanotubos de boro-nitrógeno y carbono-flúor tratada por [52] o la predicción de nuevas formas cristalográficas del carbono de [80].

\section{Método de Hartree-Fock}

En esta sección, resumimos un tipo de método numérico que permite construir el estado base de la función de ondas multi-electrón basándonos en la función de ondas del Hidrógeno (un solo núcleo) o en la función de ondas molecular de un único electron obtenida mediante el método TB.

Una aproximación comúnmente utilizada es la independencia entre electrones, aunque la interacción entre ellos se puede tener en cuenta mediante el método de Hartree. De forma similar al TB, supondremos una forma inicial de los orbitales asumiendo electrones independientes y mediante una minimización iterativa (que emplea el operador Hamiltoniano exacto), obtendremos los orbitales multi-electrón finales.

El método de Hartree no verifica el principio de Pauli, por el cuál no pueden existir dos electrones simultáneamente en el mismo estado energético. Este principio se verá satisfecho si modificamos ligeramente la función de prueba y tomamos la obtenida a partir del determinante de Slater [67], método conocido como Hartree-Fock. Aunque estos métodos proporcionan resultados fiables en problemas de Física Cuántica y Química, son computacionalmente costosos y su aplicación a sistemas muy grandes no resulta rentable.

\section{Teoría del funcional de densidad (DFT)}

Una vía alternativa al método Hartree-Fock que proporciona resultados comparables a un coste mucho menor, permitiendo la simulación de moléculas con cientos de átomos es la Teoría del funcional de densidad (DFT) [2]. Consiste en un proceso iterativo que parte de un Hamiltoniano aproximado (pero completamente determinado) relacionado con un sistema idealizado de múltiples electrones, para el cual la función de ondas exacta es conocida. Esta solución es actualizada en cada iteración optimizando el sistema ideal hasta aproximarnos cada vez más al sistema real.

Supone que el estado energético base de un sistema multi-electrón es un funcional único de la densidad de electrones $\rho$. Una fuerte simplificación de la DFT es la Teoría del funcional de densidad 
local (LDFT), que supone una densidad de electrones uniforme en todo el sistema. También se han desarrollado otros planteamientos no locales que consideran las variaciones en la densidad total, introduciendo una dependencia del estado energético base respecto al gradiente de la densidad de electrones, además de la dependencia de la densidad misma. Tu et al. [101] emplean la aproximación de densidad local planteada por [58] para simular MWNTs a tracción.

El procedimiento DFT original incluye generalmente un orden de operaciones $O\left(N^{3}\right)$, siendo N el $\mathrm{n}^{\mathrm{o}}$ de electrones, frente al orden $O\left(N^{4}\right)$ del método Hartree-Fock o tight binding.

\subsection{Modelos de dinámica molecular (MD)}

La Dinámica Molecular (MD) tuvo su aplicación inicial en Termodinámica y Química Física para calcular las propiedades termoquímicas promediadas de varios sistemas físicos incluyendo gases, líquidos o sólidos. Además ha sido aplicada recientemente a la simulación del comportamiento atómico instantáneo de un sistema material. De hecho, actualmente es uno de los métodos más aplicados en la investigación de la respuesta resistente de los CNTs.

Los modelos MD consisten en un sistema de puntos materiales o esferas sólidas sin estructura interna que interactúan entre si, de forma similar a los modelos QM, aunque en este caso cada punto representa un átomo o molécula sin considerar sus partículas constituyentes. Se puede decir entonces que la escala del elemento representativo es atómica. Las hipótesis básicas adoptadas en las simulaciones MD habituales son:

1. Las moléculas o átomos del sistema se describen como un conjunto de puntos que interactúan, cuyo movimiento viene definido por un conjunto de vectores de posición instantáneos y velocidades. La interacción depende en gran medida de la orientación espacial y de las distancias entre átomos.

2. No hay intercambios de masa en el sistema. El número de átomos permanece invariable.

El sistema simulado es tratado a menudo como un dominio aislado que conserva la energía, sin embargo existen técnicas que modelizan la disipación de energía cinética o térmica en el medio envolvente. La descripción del movimiento en términos energéticos se puede llevar a cabo en dos formas (los detalles de cada formulación pueden consultarse en [30]):

$\square$ Formulación Lagrangiana.- Las ecuaciones del movimiento se pueden expresar en términos de la función lagrangiana $\mathcal{L}$, que representa la energía total del sistema y adopta una forma:

$$
\mathcal{L}=\sum_{i=1}^{N} \frac{m_{i} \dot{r}_{i}^{2}}{2}-U\left(\mathbf{r}_{1}, \mathbf{r}_{2}, \ldots, \mathbf{r}_{N}\right)
$$

donde el primer término representa la energía cinética (es aditiva) y la función $U$ el potencial interatómico, que será nulo en un sistema de puntos independientes. Este tipo de formulación se emplea normalmente para aquellas simulaciones orientadas al análisis del movimiento atómico detallado.

$\square$ Formulación Hamiltoniana.- Dentro de esta formulación alternativa se emplea una descripción del problema en términos de las coordenadas generalizadas y la cantidad de movimiento. La función que representa en este caso la energía total (conservativa) del sistema es conocida como Hamiltoniano, y viene dado por:

$$
\mathcal{H}=\sum_{\alpha} p_{\alpha} \dot{q}_{\alpha}-\mathcal{L}
$$


donde $p_{\alpha}$ suele representar la cantidad de movimiento y $q_{\alpha}$ las coordenadas generalizadas para la partícula $\alpha$.

Si conocemos la función $\mathcal{H}$ en un estado inicial, podemos computar las posiciones instantáneas y cantidades de movimiento en los instantes siguientes resolviendo las ecuaciones del movimiento [30]. Sin embargo la formulación Hamiltoniana se utiliza con mayor frecuencia en la obtención de las características estadísticas o promedio del sistema que en la determinación del movimiento atómico detallado.

Definido el funcional energético, la evolución del sistema (posiciones y velocidades) se suele determinar mediante un procedimiento numérico de minimización para cada instante que implica habitualmente complicados algoritmos de integración en el tiempo.

Dentro de las aplicaciones de las técnicas MD en el marco de la nanotecnología, el análisis de la rigidez longitudinal de CNTs constituye una de las fundamentales. Una buena parte de autores que tratan este asunto, ensayan SWNTs y MWNTs a tracción [71], [70], [111], [123], [124] tomando un potencial armónico o tipo Tersoff-Brenner (T-B) para las interacciones de enlace y tipo LennardJones (L-J) para las interacciones de Van der Waals (VDW) (ver sección 2.4.1). En otros casos, se completa el estudio con ensayos a compresión [33], [89]. Por otra parte, para el estudio del módulo de deformación transversal se suele someter el nanotubo a torsión [45], [109].

Las técnicas MD también permiten simular los mecanismos de rotura a tracción de SWNTs [7], además de analizar las propiedades materiales de los CNTs midiendo la velocidad de las ondas sonoras al atravesar los CNTs [88] incluyendo la energía cinética, con un procedimiento algo alejado del empleo de potenciales clásicos.

Otros fenómenos como la inestabilidad a compresión o torsión [64], que denominaremos abolladura o pandeo por analogía con el estudio de sólidos homogéneos, son reproducidos fielmente por las técnicas MD [21], [91], [121], [110]. En esta línea, [43], [94] y [110] insisten en la abolladura elástica sin rotura de enlaces ni formación de defectos de Stone-Wales (situación verificada experimentalmente en [27]). Además [24], [76], [83], [90] tratan la influencia de las deformaciones mecánicas (hasta la abolladura si corresponde) sobre las propiedades eléctricas y de resistencia de los CNTs; fenómeno conocido como acoplamiento electro-mecánico, de gran interés en la fabricación de NEMS.

La elevada deformabilidad transversal de los CNTs tratada mediante MD, ya sea entre tubo y sustrato [38] o entre SWNTs [96], pone de manifiesto una marcada anisotropía de los nanotubos como conjunto, a pesar de la isotropía 2D que presenta la lámina de grafeno en su propio plano.

Teniendo en cuenta la gran fiabilidad que presentan los modelos MD, también se han empleado para validar otros modelos como los modelos lámina [103], [115] simulando CNTs a compresión, flexión y torsión hasta la abolladura.

Sin embargo, la exigencia computacional de estos métodos es considerable debido al gran número de grados de libertad implicados (del orden $O(6 N)$ ), sumado a la necesidad de integración en el tiempo. En consecuencia, las simulaciones MD se suelen limitar a un pequeño número de átomos, sustituyendo el medio envolvente por condiciones de contorno rígidas o periódicas. Por tanto, el comportamiento físico del elemento de volumen representativo no puede extrapolarse exactamente al sistema macroscópico de interés. Debido al efecto de superficie de las condiciones de contorno periódicas, una simulación puede considerarse válida solo cuando el tamaño del dominio simulado es mayor que el radio de corte del potencial y que el rango efectivo del fenómeno bajo investigación [67]. En este sentido, la potencia de los ordenadores modernos permite modelizar dominios con un máximo de varios cientos de millones de átomos $\left(10^{8}-10^{9}\right.$ átomos en orden de magnitud [67], [108]), por tanto estos métodos están limitados a un tamaño de muestra de $0,1 \times 0,1 \times 0,1 \mu \mathrm{m}$. Recordemos que $1 \mathrm{Mol}$ de $\mathrm{C}$ (equivalente a $12 \mathrm{~g} \mathrm{de} \mathrm{C}$ ) contiene $6,022 \cdot 10^{23}$ átomos.

Por otra parte, los tiempos de simulación MD suelen estar en el orden de los picosegundos, bastante alejados de los tiempos representativos en un proceso de carga cuasi-estático, necesario en el 
comportamiento macroscópico del sistema. Por esto y por las limitaciones de potencia computacional, la modelización atómica a gran escala resulta prohibitiva.

\subsection{Modelos de mecánica estructural molecular (MSM)}

Un tipo de modelos de concepción similar a los modelos MD, aunque con una perspectiva del problema más cercana a la ingeniería estructural, son los modelos de Mecánica Molecular o también denominados de Mecánica Estructural Molecular (MSM). El fundamento de estos modelos consiste en la minimización de una función potencial definida, desde un punto de vista estático, con el objetivo de encontrar una nueva posición de equilibrio bajo la acción de ciertas cargas externas. Al tratarse de un problema estático, podemos convertir cada uno de los tipos de interacción derivados de la función potencial en un elemento estructural de rigidez equivalente. Describimos a continuación los distintos modelos MSM definidos en la literatura.

Una primera descripción del sistema real [61] muy cercana a los sistemas estructurales clásicos de barras, es convertir el entramado de enlaces de un SWNT en un sistema de barras reticuladas con rigideces a axil, flexión y torsión equivalentes derivadas de un potencial armónico, donde los nudos representan los átomos de C. Los mismos autores [59] extienden dicho modelo a MWNTs de dos capas, manteniendo la estructura reticulada en cada pared y adoptando un sistema de bielas equivalentes para plasmar la interacción de VDW (mediante un potencial tipo L-J) entre capas. Este modelo ha sido empleado también para el análisis dinámico de SWNTs [60] y MWNTs [62], [63]. Más recientemente, otros autores [9], [100] han trabajado sobre el modelo de barras reticuladas aportando sólo ligeras modificaciones. Sin embargo, [120] utiliza más eficazmente el modelo reticulado como punto de partida para la validación con otros modelos continuos y el estudio de sistemas más complejos, tales como los fajos de SWNTs, los propios MWNTs o el buckypaper (papel poroso fabricado a partir de SWNTs formando una malla desordenada).

A pesar del intensivo trabajo dedicado a este tipo de modelos por ser muy intuitivos desde el punto de vista estructural, permitir el uso de software stándar y aportar resultados razonables, resulta bastante controvertido asignar resistencias de flexión y torsión a un enlace covalente formado por un grupo de electrones compartido entre dos núcleos atómicos de $\mathrm{C}$.

Por otra parte, Chang y Gao [14] siguen una línea conceptualmente diferente que reproduce la interacción eminentemente longitudinal entre cada par de átomos adyacentes de $\mathrm{C}$ mediante elementos tipo biela que funcionarán exclusivamente a axil. Asimismo, introduce resortes angulares en el entorno diferencial de cada nudo reproduciendo indirectamente la interacción de tres cuerpos que está asociada a distorsiones angulares entre enlaces covalentes. Empleando este modelo, denominado spin-bar o stick-spiral, simula el comportamiento a tracción de SWNTs considerando la curvatura para incluir el efecto del diámetro en las características mecánicas longitudinales. La misma cuestión se trata en [114], pero basándose en el potencial de Morse y llegando a un proceso iterativo para la obtención de resultados. Más tarde, Chang et al. [15] y Ansari et al. [2] extienden el trabajo de [14] para analizar la influencia de la quiralidad y el diámetro en las propiedades mecánicas de los SWNTs, incluso [16] extienden este modelo al análisis de deformaciones críticas longitudinales.

Hasta este punto, todas las referencias relativas al stick-spiral hacen desarrollos simplificados de las ecuaciones del modelo, incluyendo unos pocos átomos y sus enlaces intermedios bajo la acción de una configuración de cargas específica. Por tanto, se puede comprobar la falta de una formulación general válida para cualquier estado de carga. No obstante, Meo y Rossi [74] procesan este modelo de forma global mediante el código comercial de elementos finitos $A N S Y S^{\circledR}$. Se adopta un potencial de interacción de Morse para simular SWNTs a tracción, pero linealizando la interacción relativa a la distorsión angular entre enlaces. En esta línea, la referencia [11] estudia el comportamiento frente a distintos tipos de inestabilidad mediante el código Materials Studio haciendo uso del mismo modelo de MM. 
Además, el concepto de preenergía o deformaciones iniciales tratado en las técnicas MD, no aparece tratado explícitamente en las referencias relativas a la mecánica molecular, incluso aunque la curvatura del tubo se incluya mediante sofisticados razonamientos trigonométricos.

Citamos además otros modelos, que basándose en técnicas MSM y empleando sistemas de barras equivalentes, realmente se sitúan en un punto intermedio entre los modelos MD y los cuasi-continuos (QC) (sección 2.5.2) basados en la regla de Cauchy-Born. Por ejemplo, Odegard [79] define un modelo de barras articuladas, algunas de ellas atravesando la célula hexagonal elemental para reproducir indirectamente la distorsión angular entre enlaces. También [78], [77], [106] proponen modelos tipo stick-spiral estudiando un elemento de volumen representativo formado por una célula hexagonal plana (desprecian el efecto de la curvatura) y aplicando ciertas condiciones de simetría dependiendo del estado de cargas aplicadas, lo cual limita el campo de aplicación del modelo.

Teniendo en cuenta las consideraciones anteriores, adoptamos en esta tesis el modelo MSM denominado stick-spiral enfocado a un desarrollo completamente general de sus formulación, válido para cualquier estado de carga y que incluya explícitamente ciertas deformaciones iniciales asociadas a la curvatura del cilindro desde su posición de equilibrio en la lámina plana de grafeno. Se describen a continuación las principales características de la formulación del modelo desarrollada en esta tesis, en comparación con las técnicas MD:

1. No se requiere tener en cuenta en la formulación la transmisión de calor con el medio envolvente, luego los modelos MM sacrifican algo de fiabilidad y precisión a cambio de simplificar el problema. Realmente, fenómenos como la inestabilidad vienen altamente influenciados por la fluctuación térmica aleatoria de los átomos en torno a su posición de equilibrio. A pesar de ello, el acoplamiento entre la vibración térmica y los defectos estructurales dificultan el análisis de la inestabilidad intrínseca por métodos MD. En este sentido, los modelos MSM pueden ser una herramienta útil como primer escalón para entender las respuestas básicas a pandeo o abolladura.

2. Los modelos MSM son fundamentalmente estáticos y no requieren integración en el tiempo.

3. Para el mismo número $N$ de partículas en el sistema, el modelo stick-spiral sólo requiere los $3 N$ desplazamientos lineales en los nudos como variables cinemáticas fundamentales, frente a las $6 N$ (velocidades y posiciones) necesarias en los MD, lo cual permite un mayor rendimiento numérico de los algoritmos desarrollados.

\subsubsection{Potenciales interatómicos}

El potencial interatómico constituye la parte de la energía total del sistema (ya sea $\mathcal{L}$, ec (2.1) o $\mathcal{H}$, ec (2.2) que reproduce la interacción mecánica entre átomos, presente tanto en los modelos MD como en los MSM. La forma general de esta función potencial es:

$$
U\left(\mathbf{r}_{1}, \mathbf{r}_{2}, \ldots, \mathbf{r}_{N}\right)=\sum_{i} V_{1}\left(\mathbf{r}_{i}\right)+\sum_{i, j>i} V_{2}\left(\mathbf{r}_{i}, \mathbf{r}_{j}\right)+\sum_{i, j>i, k>j} V_{3}\left(\mathbf{r}_{i}, \mathbf{r}_{j}, \mathbf{r}_{k}\right)+\cdots
$$

donde: $V_{1}\left(\mathbf{r}_{i}\right)$ representa la energía debida a un campo de fuerzas externas.

$V_{2}\left(\mathbf{r}_{i}, \mathbf{r}_{j}\right)$ es la interacción de dos cuerpos.

$V_{3}, V_{4}, \cdots$ son los términos de interacción multicuerpo. Sobre todo se conceptúan para reproducir los cambios en la geometría del entramado de átomos debido a las variaciones angulares. Los potenciales $V_{3}$ suelen resultar complicados e incapaces de describir todas las posibles configuraciones de enlace, mientras los potenciales $V_{4}$ y siguientes aparecen como intratables. La solución consiste en desarrollar potenciales de dos cuerpos modificados que consideren indirectamente la variación angular. 
Los potenciales interatómicos aplicables a sistemas formados por átomos de C o Si vienen expresados por funciones que pueden ser clasificadas en dos géneros:

$\square$ Funciones armónicas. Describen el potencial molecular como la adición de los potenciales correspondientes a los diferentes desplazamientos relativos entre átomos ([38], [45], [54], [59], [61], [70], [71], [78], [79], [106]), cada uno de ellos expresado como una forma cuadrática del desplazamiento relativo. La expresión general para estos potenciales es del tipo:

$$
U=\sum U_{r}+\sum U_{\theta}+\sum U_{\phi}+\sum U_{\omega}+\sum U_{v d w}
$$

donde:

$$
\begin{array}{ll}
U_{r}=\frac{1}{2} k_{r}(\Delta r)^{2} & \begin{array}{l}
\text { Potencial debido a la variación de longitud del enlace } \\
U_{\theta}=\frac{1}{2} k_{\theta}(\Delta \theta)^{2}
\end{array} \\
\begin{array}{l}
\text { Potencial debido a la variación angular entre enlaces contiguos en } \\
\text { la lámina de grafeno }
\end{array} \\
U_{\phi} & \begin{array}{l}
\text { Potencial debido a la torsión del enlace } \\
U_{\omega}=\frac{1}{2} k_{\omega}(\Delta \omega)^{2}
\end{array} \\
U_{v d w} & \text { Potencial debido a la inversión del enlace (giro fuera del plano) } \\
\text { Potencial debido a la interacción de Van der Waals }
\end{array}
$$

Las constantes $k_{r}, k_{\theta}$ y $k_{\omega}$ representan las rigideces que se oponen a las diferentes formas de redistribución atómica. En el caso de SWNTs aislados, los dos términos de mayor importancia en (2.4) son $U_{r}$ y $U_{\theta}$, despreciándose el resto ([14], [54], [78], [106]) y llegando a una expresión del potencial simplificada:

$$
U=\sum \frac{1}{2} k_{r}(\Delta r)^{2}+\sum \frac{1}{2} k_{\theta}(\Delta \theta)^{2}
$$

Si se pretende mayor precisión, se suele añadir los términos de inversión y torsión, teniendo este último una interpretación física algo dudosa. La expresión adoptada habitualmente [14], [45] en los cálculos es del tipo:

$$
U_{\phi}=\frac{1}{2} k_{\phi}\left(1+\cos \left[n \phi-\phi_{0}\right]\right)
$$

donde $\phi_{0}$ es el ángulo de equilibrio para este tipo de enlaces, $n$ es la periodicidad del enlace y $k_{\phi}$ es una constante.

La expresión (2.5) implica un comportamiento elástico lineal de todos sus componentes, cuyo ejemplo más representativo es el potencial tipo AMBER2, ampliamente utilizado [20], [61], [59], [78], [79], [106] para modelizar la interacción entre átomos de C dentro de la propia lámina de grafeno o SWNT y cuyos parámetros adoptan los valores:

$$
\begin{aligned}
\frac{k_{r}}{2} & =3.26 \cdot 10^{-7} \frac{\mathrm{nJ}}{\text { enlace } \cdot \mathrm{nm}^{2}}=46900 \frac{\mathrm{Kcal}}{\mathrm{mol} \cdot \mathrm{nm}^{2}} \\
\frac{k_{\theta}}{2} & =4.38 \cdot 10^{-10} \frac{\mathrm{nJ}}{\text { angulo } \cdot \mathrm{rad}^{2}}=63 \frac{\mathrm{Kcal}}{\mathrm{mol} \cdot \mathrm{rad}^{2}}
\end{aligned}
$$

y tras los oportunos cambios de unidades:

$$
k_{r}=652 \frac{n N}{\text { enlace } n m} \quad k_{\theta}=0.876 \frac{n N \cdot n m}{\text { angulo } \cdot r a d}
$$

\footnotetext{
${ }^{2}$ Assisted Model Building with Energy Refinement, se trata de un conjunto de campos de fuerzas de mecánica molecular para la simulación de biomoléculas, empleados en una gran cantidad de programas de simulación y de dominio público en el campo de la bioquímica.
} 
También dentro de las funciones armónicas, aunque sin tratarse de una forma cuadrática, podemos citar el potencial de dos cuerpos tipo Lennard-Jones 6 - 12 (L-J) [46], [47], usado habitualmente para modelizar la interacción de Van der Waals (VDW) entre láminas de grafeno, capas de MWNTs o fajos de SWNTs considerados como un conjunto [87], [93]. El potencial L-J adopta la forma:

$$
V\left(\mathbf{r}_{i}, \mathbf{r}_{j}\right)=V(r)=4 \varepsilon\left[\left(\frac{\sigma}{r}\right)^{12}-\left(\frac{\sigma}{r}\right)^{6}\right]
$$

donde $r$ es la distancia entre cada par de átomos, $\sigma=0.3345 \mathrm{~nm}$ es la distancia de equilibrio y $\varepsilon=3.19 \cdot 10^{-3} \mathrm{eV}$ es una constante que representa la energía de enlace.

$\square$ Potenciales multicuerpo. Incluyen en la interacción entre cada dos átomos vecinos (enlace covalente) el efecto del resto de átomos vecinos no considerados en cada dímero C-C. Esto se traduce en que los términos de dos cuerpos de la expresión general del potencial (2.3) dependerán tanto de la distancia entre los dos átomos objeto de estudio, como de los ángulos que formarán los enlaces covalentes vecinos con el enlace principal.

La función empleada más extensamente ([6], [21], [24], [33], [39], [40], [43], [44], [64], [89], [103], [110], [111], [115], [121], [122]) para representar la interacción atómica en compuestos covalentes formados por C (grafito, diamante, CNTs) es el potencial desarrollado por Tersoff [95] para el Si, y parametrizado experimentalmente por Brenner [10] para compuestos de C. El potencial total tipo Tersoff-Brenner (T-B) se puede escribir:

$$
E_{b}=\frac{1}{2} \sum_{i} E_{i}
$$

siendo $E_{i}$ la contribución al potencial total de cada localización atómica, que tendrá una expresión:

$$
E_{i}=\sum_{j \neq i}\left[V_{R}\left(r_{i j}\right)-B_{i j} V_{A}\left(r_{i j}\right)\right]
$$

donde $r_{i j}$ es la distancia entre los átomos $i$ y $j$, y los términos $V_{R}\left(r_{i j}\right)$ y $V_{A}\left(r_{i j}\right)$ son los términos repulsivo y atractivo respectivamente, dados por las funciones:

$$
\begin{aligned}
& V_{R}\left(r_{i j}\right)=f_{i j}\left(r_{i j}\right) \frac{D_{i j}^{(e)}}{\left(S_{i j}-1\right)} e^{-\sqrt{2 S_{i j}} \beta_{i j}\left(r_{i j}-R_{i j}^{(e)}\right)} \\
& V_{A}\left(r_{i j}\right)=f_{i j}\left(r_{i j}\right) \frac{D_{i j}^{(e)}}{\left(S_{i j}-1\right)} e^{-\sqrt{2 / S_{i j}} \beta_{i j}\left(r_{i j}-R_{i j}^{(e)}\right)}
\end{aligned}
$$

La función $f_{i j}\left(r_{i j}\right)$ es una función de corte que restringe el potencial a los átomos vecinos más próximos y viene dada por:

$$
f_{i j}\left(r_{i j}\right)= \begin{cases}1, & r_{i j}<R_{i j}^{(1)} \\ \frac{1}{2}\left[1+\cos \left(\frac{\pi\left(r_{i j}-R_{i j}^{(1)}\right)}{\left(R_{i j}^{(2)}-R_{i j}^{(1)}\right)}\right)\right], & R_{i j}^{(1)}<r_{i j}<R_{i j}^{(2)} \\ 0, & r_{i j}>R_{i j}^{(2)}\end{cases}
$$

La función $B_{i j}$ introduce en el potencial la dependencia del entorno atómico y se puede escribir:

$$
B_{i j}=\left[1+\sum_{k(\neq i, j)} G_{i}\left(\theta_{i j k}\right) f_{i k}\left(r_{i k}\right) e^{\alpha_{i j k}\left[\left(r_{i j}-R_{i j}^{(e)}\right)-\left(r_{i k}-R_{i k}^{(e)}\right)\right]}\right]^{-\delta}
$$


donde $G_{i}\left(\theta_{i j k}\right)$ es una función que depende del ángulo entre los enlaces $i-j$ e $i-k$, adoptando una expresión:

$$
G(\theta)=a_{0}\left\{1+\frac{c_{0}^{2}}{d_{0}^{2}}-\frac{c_{0}^{2}}{\left[d_{0}+(1+\cos \theta)^{2}\right]}\right\}
$$

Una descripción más detallada del resto de parámetros y del ajuste experimental de sus valores puede encontrarse en [95].

Si tomamos $\beta_{i j}=2$ en (2.12) (ver [10], [95]), los términos de dos cuerpos se convierten en el potencial tipo Morse. De hecho, Belytschko et. al. [7] plantean un potencial de Morse modificado que aproxima el T-B razonablemente bien hasta deformaciones longitudinales del $10 \%$ y viene dado por:

$$
\begin{aligned}
E & =\sum E_{r}+\sum E_{\theta} \\
E_{r} & =D_{e}\left\{\left[1-e^{-\beta(\Delta r)}\right]^{2}-1\right\} \\
E_{\theta} & =\frac{1}{2} k_{\theta}(\Delta \theta)^{2}\left[1+k_{s}(\Delta \theta)^{4}\right]
\end{aligned}
$$

con unos valores para los parámetros implicados:

$$
\begin{array}{ll|}
\hline D_{e}=1.807 \mathrm{eV} & \beta=38.43 \mathrm{~nm}^{-1} \\
k_{\theta}=5.617 \mathrm{eV} & k_{s}=0.754 \mathrm{rad}^{-4} \\
\hline
\end{array}
$$

Este potencial es preferido en algunos casos [74], [77], [114] por su facilidad de implementación, ya que desacopla la contribución de los alargamientos de enlace 2.17) de la correspondiente a las distorsiones angulares (2.18).

\subsection{Métodos de simulación multiescala (MS)}

En las últimas décadas, la investigación en la modelización de materiales ha venido dominada por los modelos continuos, que implícitamente promedian la dinámica a escala atómica y la evolución de los defectos en el tiempo y el espacio. En consecuencia, los modelos continuos sólo resultan válidos para sistemas suficientemente grandes que incluyan un número sustancial de defectos. Por eso, numerosas observaciones experimentales del comportamiento material de los CNTs no tienen una explicación razonable en el marco de la mecánica del continuo.

Teniendo en cuenta las respectivas limitaciones de los modelos discretos o atómicos y de los modelos continuos, una forma asequible computacionalmente de tratar sistemas de gran número de átomos sin perder mucha precisión es utilizar métodos de simulación multiescala (MS) [67], [86]. En las regiones del dominio donde se requiere una gran precisión en captar el movimiento de cada átomo individualmente (escala detallada) se utiliza un método atómico de simulación (MD o QM), mientras el resto del dominio se simula siguiendo una simulación continua (escala grosera) donde se considera una deformación suave y homogénea.

Dependiendo de la forma de intercambiar información entre las zonas de escala grosera y escala detallada, los métodos multiescala se pueden clasificar en jerárquicos y simultáneos.

\subsubsection{Métodos MS jerárquicos}

Incluyen las propiedades atómicas intrínsecas del sólido en la formulación continua de acuerdo con la regla de Cauchy-Born, de forma que las escalas detalladas son dependientes de las groseras de 
alguna forma definida y predecible, al estilo de una "muñeca rusa". Estos métodos son más efectivos para fenómenos elásticos en problemas de una única fase [67]. Las dificultades surgen típicamente de la modelización de defectos en los entramados atómicos, dislocaciones y fenómenos de fractura.

Definición 2.5.1 (Regla de Cauchy-Born) El comportamiento mecánico de cualquier celda representativa hexagonal del entramado se puede asimilar al de una región infinitesimal equivalente del continuo alrededor del punto considerado. En particular, dicha celda se deforma de acuerdo con un gradiente continuo y localmente uniforme de deformación. Por tanto, la densidad de energía de deformación en un punto del continuo se puede aproximar por un modelo atómico.

\subsubsection{Métodos MS simultáneos}

El comportamiento de cada región, simulado a una escala diferente, depende fuertemente del resto a través de una interacción sensiblemente equitativa, es decir, en la que ninguna región domina el comportamiento de las demás. Se resuelve simultáneamente un modelo apropiado a cada región, mientras se introduce un ligero acoplamiento entre las distintas escalas. La dependencia entre escalas es complicada y no está predefinida. Son más apropiados para estudiar problemas complicados, incluyendo deformaciones heterogéneas del entramado, fracturas en materiales macroscópicos de varias fases, y nanofluidos. Los más conocidos hasta el momento son los MAAD, cuasi-continuos y BSM.

\section{Macroscopic, atomistic and ab initio dynamics (MAAD)}

Los métodos consistentes con las distintas escalas son enlazados simultáneamente para simular la propagación de la rotura en materiales frágiles [67]. Los Modelos TB se emplean para simular la rotura de enlaces atómicos, los modelos MD se emplean en la región que envuelve el frente de rotura, y el método de los elementos finitos (FEM) se emplea en regiones lejanas a la rotura, donde la deformación generalmente es suave. La dinámica del sistema completo viene gobernada por una función Hamiltoniana total que combina los Hamiltonianos individuales de las tres regiones.

Un inconveniente de estos métodos es la inexistencia de estudios rigurosos que cuantifiquen la eficacia de este método para eliminar reflexiones de ondas espurias en los contornos de simulación.

\section{Métodos cuasi-continuos (QC)}

Se mantiene el concepto de medio continuo y de partícula, mientras la ley constitutiva se reemplaza por una obtenida a partir de cálculos atómicos directos. Cada partícula del continuo se considera como un pequeño cristal envolviendo a un átomo o elemento representativo. La energía de deformación asociada con el átomo representativo se puede evaluar sumando el potencial interatómico para todos los átomos de su entorno siguiendo la regla de Cauchy-Born (Definición 2.5.1).

Podemos citar como ejemplo [113], donde se estudia la flexión y estado tensional de vigas constituidas por nanocomposites en función de la distribución geométrica (orientada o aleatoria) y fracción en volumen de los CNTs de refuerzo.

Los modelos QC locales, como es el caso de [77], [78], [79], [106], se pueden incluir en esta sección debido a la adopción implícita de la regla de Cauchy-Born. No obstante, al adoptarse un sistema de barras y muelles en los cálculos atómicos directos se citaron también en la sección 2.4

Otros autores, adoptan un modelo QC local empleando potenciales interatómicos en los cálculos a nivel atómico. En particular, [6] simula MWNTs a flexión y torsión hasta la abolladura introduciendo la preenergía (energía almacenada por deformación desde la lámina de grafeno plana hasta el nanotubo), estableciendo que es proporcional a $1 / r^{2}$.

Por otra parte, [44] y [122] matizan la regla de Cauchy-Born afirmando que su aplicabilidad a sistemas atómicos requiere simetría central de la molécula, condición que no verifican los CNTs. 
Hablando estrictamente, no se puede aplicar la regla de Cauchy-Born a un CNT con deformación supuestamente uniforme en su conjunto, ya que sobre la unidad básica se pueden producir deformaciones no homogéneas. Para evitar esta dificultad, descomponen el entramado hexagonal en dos entramados triangulares (de forma que cada átomo de un entramado está rodeado por tres átomos vecinos pertenecientes al otro) e introducen un vector decalaje $\boldsymbol{\xi}$ que transforma un entramado triangular en el otro. De esta forma podemos asignar un único mapeo uniforme de deformación (tensor gradiente de la deformación $\mathbf{F}$ ) dentro de la celda hexagonal.

\section{Método de conexión de escalas (Bridging Scale Method, BSM)}

Las soluciones MEF y MD existen simultáneamente en el dominio computacional completo, y los cálculos MD son efectuados solo en las regiones donde sean necesarios [67]. La localización de la zona de escala detallada puede ser determinada por un análisis multi-resolución de la simulación a escala grosera, convirtiendo el esquema en adaptativo. Resulta útil para analizar la abolladura local o rippling mode de deformación de CNTs.

\subsubsection{Condiciones de contorno multiescala}

La cuestión clave en un planteamiento simultáneo es el acoplamiento entre las escalas grosera y fina. Se requiere una aproximación en la interfaz fino-grosero debido a la incompatibilidad fundamental entre las descripciones atómica y continua, impuesta por el desfase de características de dispersión de los medios discreto y continuo en simulaciones MD, y por el carácter no local de la interacción atómica tanto en simulaciones dinámicas como cuasi-estáticas.

La mayoría de métodos simultáneos (salvo el BSM) incluyen una zona artificial de compromiso entre ambas escalas, donde existen pseudo-átomos disponibles en la parte continua de la interfaz y participan del espacio físico junto con los Elementos Finitos. Hacia la zona de contacto entre escalas, los Elementos Finitos deben ser escalados hasta la longitud de enlace químico o iónico, lo que puede producir matrices de rigidez mal condicionadas. El objetivo de esta zona es asegurar un acoplamiento suave entre los regímenes atómico y continuo.

El grupo de pseudo-átomos sirve para eliminar la superficie sin sentido físico entre zonas del entramado atómico, de forma que los átomos reales tengan un conjunto completo de vecinos interactuando en el dominio continuo. En simulaciones dinámicas, la región de compromiso también sirve como absorbente/amortiguador para reducir la reflexión espuria de fonones de alta frecuencia que no pueden llegar a la zona de escala grosera.

Las formulaciones armónicas de las condiciones de contorno multiescala incluidas en [67] sólo tratan correctamente no linealidades débiles en el comportamiento atómico de la interfaz. Para tratar no linealidades más importantes, es necesario introducir un término no armónico considerado como una perturbación del modelo lineal (ver referencias en [67]).

\subsection{Modelos híbridos atómico-continuos (HAC)}

Un tipo de modelos empleados menos frecuentemente consiste en los modelos HAC. Este tipo de métodos trata mediante técnicas MD la interacción de VDW entre capas de MWNTs (o bien entre SWNTs dentro de un fajo) y asume un comportamiento continuo en cada capa a través de un modelo lámina o de elementos finitos $2 \mathrm{D}$.

Por ejemplo, [81] trata mediante Elementos Finitos 2D cada capa de los MWTNs y los SWNTs, reproduciendo la interacción de VDW entre capas, o entre tubo y sustrato, mediante el potencial L-J. Esta última se traduce a un sistema de bielas, procesando el conjunto en el código comercial $A B A Q U S{ }^{\circledR}$. Todo ello le permite efectuar una buena cantidad de simulaciones, como la flexión 
de SWNTs hasta abolladura, deformabilidad transversal entre SWNTs o con un sustrato y flexión de MWNTs hasta abolladura. Con un modelo similar, [18] estudia los modos de vibración libre de MWNTs modificando secuencialmente las condiciones de contorno de cada capa en uno de sus extremos. Análogamente, [104] trata los SWNTs como láminas continuas aisladas y la interacción entre ellos mediante un potencial L-J (6-12) con el objetivo de ensayar el desfase longitudinal entre SWNTs en un fajo, así como su deformabilidad transversal. Esta deformabilidad resulta clave en la anisotropía del conjunto.

Los métodos HAC se diferencian de los métodos multiescala (MS) en que los últimos adoptan técnicas MD en una región localizada y el resto del dominio es tratado como continuo, acoplando ambas soluciones en una región de compromiso; mientras que los primeros desacoplan las interacciones que afectan poco al comportamiento conjunto (como las VDW) del resto, y no incluyen regiones de compromiso que puedan dificultar el intercambio de información entre fuerzas internas y condiciones de contorno.

\subsection{Modelos continuos}

\subsubsection{El modelo viga de Navier}

Dadas las configuración geométrica alargada de los CNTs, un buen número de estudios han adoptado como modelo de análisis la viga de Navier sin consideraciones adicionales. La sencillez que proporciona en el tratamiento de resultados experimentales junto con la intención de buena parte de los autores de definir un módulo de Young longitudinal para estas moléculas tubulares, ha favorecido el uso de este modelo frente a otros más fiables.

En la búsqueda de la determinación de las características mecánicas de los CNTs, una primera aproximación consiste en su manipulación directa sometiéndolos a esfuerzos y/o deformaciones. Podemos citar a [112], que ensaya MWNTs en ménsula sometidos a una carga puntual perpendicular aplicada con la punta de un AFM a diferentes distancias y compara los resultados con el modelo viga, obteniendo $E=1.28 \pm 0.59 \mathrm{TPa}$. Por otra parte, [119] ensaya MWNTs a tracción directa entre dos puntas de AFM, asumiendo un tratamiento continuo longitudinal. Obtiene $E=270-950 \mathrm{GPa}$ y una tensión de rotura $\sigma_{r}=11-63 \mathrm{GPa}$ a una deformación del $12 \%$ para la capa exterior, que recibe directamente los esfuerzos. Sin embargo, asegura que los valores obtenidos pueden estar distorsionados por la falta de transmisión eficaz de carga entre capas del MWNT y por el modo de fallo espadavaina. En esta línea, [118] ensaya análogamente fajos de SWNTs a tracción con AFM, obteniendo $E=320-1470 \mathrm{GPa}$ y $\sigma_{r}=13-52 \mathrm{GPa}$ siempre que los SWNTs del perímetro asuman toda la carga (si la carga se considerara uniformemente distribuida entre todos los SWNTs, los valores resultantes serían aún menores). Afirman que el motivo por el cual se obtienen valores experimentales reducidos podría ser el posible deslizamiento de las puntas del AFM respecto a los SWNTs.

Otra vía de obtención de las características mecánicas consiste en someter a los CNTs a vibraciones térmicas, comparando las frecuencias de vibración reales, con las proporcionadas por el modelo viga de Navier. En esta dirección [99] obtiene $E=3.7 \pm 0.2 \mathrm{TPa}$ con un valor medio de $E m=1.8 \mathrm{TPa}$ para MWNTs en ménsula, [50] obtiene $E=0.9-1.7$ TPa para SWNTs y [17] establece $E=1.22 \pm 0.24$ TPa para nanotubos de boro-nitrógeno (BN) en ménsula sometidos a vibraciones dinámicas. También [116] obtienen los modos libres de vibración para un nanotubo bicapa mediante un modelo viga de Navier no lineal geométrico sin amortiguamiento.

Extendiendo el campo de aplicación del modelo viga de Navier, [82] emplea un modelo 1D continuo para analizar el comportamiento a compresión de elementos alargados embebidos en la matriz de un composite, incluyendo la interacción con el material matriz. Concluye que la inestabilidad por pandeo suele ser el fallo dominante incluso en composites de elementos con muy alta rigidez longitudinal. Asimismo, [12] utiliza la viga de Navier (y la lámina cilíndrica) para estudiar el pandeo 
de nanotubos y "nanovainas" biempotrados a compresión, incluyendo la interacción de VDW entre capas y con la partícula contenida en los tubos.

Otros autores [24], emplean el modelo viga de Navier para comparar con otros modelos, como los MD. En este caso se simula SWNTs biempotrados sometidos a flexión bajo la acción de un campo eléctrico, para estudiar la interacción entre las respuestas mecánica y eléctrica.

Experimentalmente, [93] estudian el comportamiento de fajos de SWNTs a flexión sujetos a un sustrato con relieve y cargados con la punta de un AFM. El tratamiento de datos se efectúa con una viga de Navier de sección circular equivalente, pero afirman que el ensamblaje de SWNTs se debe considerar como una viga anisótropa por posible deslizamiento entre ellos. Obtienen unos valores (considerando el fajo de SWNTs como conjunto) para el módulo de Young de $E_{r}=600 \mathrm{GPa}$ y para módulo de rigidez transversal de $G_{r}=19.5 \mathrm{GPa}$. Adicionalmente, [87] plantea para el mismo problema anterior un modelo viga de Navier modificado donde incluye la influencia de la transmisión del rasante entre SWNTs (mediante un coeficiente de eficacia) en la resistencia a flexión del fajo. Concluye, como [93], que la hipótesis de un fajo de SWNTs como viga homogénea no es válida.

\section{Comentarios sobre los parámetros elásticos adoptados en modelos continuos}

A partir de los valores aportados en las referencias consultadas, observamos una gran dispersión en los resultados obtenidos (ya sean numéricos o experimentales) para el módulo de Young, variando desde un valor aproximado de $1 \mathrm{TPa}$ ([52], [77], [78], [88] entre otros) hasta los 5.5 TPa de [99] y [115]. En el coeficiente de Poisson la variabilidad es menor, rondando la mayoría de valores en torno a $v=0.15-0.3$ (por ej. [50], [52]), con un promedio muy cercano al $v=0.19$ del grafeno.

Esta dispersión en los valores de $E$ viene condicionada directamente por el espesor de lámina equivalente adoptado para cada capa de grafeno formando un SWNT o contenida en un MWNT. Una buena parte de autores (por ej. [37], [50]) asignan un espesor $h=0.34 \mathrm{~nm}$ (espaciado interplanar del grafito) arbitrariamente. De hecho [71] afirma que los MWNTs presentan experimentalmente un interespaciado de $t=0.34 \mathrm{~nm}$, situación que limita las posibles combinaciones de capas simples para formar un MWNT. En el otro extremo, [121] estudian la estabilidad de MWNTs bicapa forzando las quiralidades de cada capa y analizando la influencia del espaciado resultante en el comportamiento a compresión.

En general se asume que la superficie media de la lámina equivalente es suave y viene generada a partir de la posición de equilibrio de los átomos de $\mathrm{C}$, aunque los enlaces covalentes no descansen necesariamente sobre dicha superficie. El material continuo representa a los átomos discretos y las nubes de electrones que los envuelven, siendo el espesor equivalente de pared $h$ una variable controvertida que pretende reproducir la contribución del conjunto de partículas implicadas a la rigidez del modelo. La separación entre capas de grafeno proporciona una cota superior para $h$.

Otros enfoques [81], [101], [115] consideran el CNT como una lámina equivalente, computan (mediante cálculos ab initio [52], [101], MD o experimentalmente) las rigideces a axil y a flexión de dicha lámina y forzando las relaciones de rigidez habituales de la teoría clásica de láminas obtienen $E=5.5 \mathrm{TPa}$ y $h=0.066 \mathrm{~nm}[115]$. Por tanto se puede concluir que los valores de $E$ extremadamente elevados vienen asociados a espesores asignados de lámina inusualmente reducidos. Odegard et al. [79] refuerza la arbitrariedad en la elección del espesor, afirmando que $h$ tiene una cierta dependencia del tipo de carga al que se somete la lámina.

Una manera de evitar la dificultad en la asignación del espesor de pared [52], [108] consiste en considerar las rigideces de nuestro modelo continuo (viga o lámina) como parámetros independientes entre sí, sin recurrir a las fórmulas clásicas que los relacionan con $E, v$ y $h$.

Siguiendo esta orientación, es posible introducir la abolladura considerando unas rigideces eficaces parametrizadas convenientemente [66], [108] en función del nivel de deformación. Se define entonces el módulo de Young eficaz $E_{e f}$ y se estudia la variación del ratio $E_{e f} / E$ en función del diámetro, aunque $E_{e f}$ es de dudoso sentido físico, ya que [108] convierte una no linealidad geométrica 
en una no linealidad mecánica.

\section{Aplicabilidad de los modelos continuos}

Esta incertidumbre respecto a los parámetros elásticos a adoptar, induce a pensar que los modelos continuos no tienen aplicabilidad directa en el análisis estructural de CNTs, salvo añadiendo ciertas consideraciones adicionales. De hecho, [32] aportan razonamientos sencillos para determinar la aplicabilidad del modelo viga en la estimación del módulo de Young a través de la simulación de MWNTs a tracción y flexión mediante una viga de Navier. Se concluye que serían necesarias infinitas capas con un espesor mucho más reducido que la separación interplanar para validar la hipótesis de sección transversal continua. En particular, se requiere $n=201$ capas y $t<<s$ para acotar el error en la estimación de $E$ mediante el modelo continuo a $\pm 1 \%$. Además, si adoptamos una separación de $0.34 \mathrm{~nm}$, el módulo de Young no se puede considerar una propiedad material, ya que depende de la geometría.

Incluso cumpliendo las condiciones anteriores, tratar un CNT como una viga de Navier es bastante cuestionable, ya que la existencia de las innumerables celdas hexagonales provocará una deformación por cortante del nanotubo como pieza no despreciable. En este sentido, parece más razonable tratar los nanotubos como vigas de Timoshenko, con una adecuada parametrización de las rigideces a la distorsión angular (no tratada en las referencias consultadas).

Una de las hipótesis clave de la teoría del continuo es que el número de átomos del material implicado sea considerado infinito para poder promediar sus propiedades y homogeneizar el sólido. Este criterio es expuesto por [34] para CNTs, afirmando que se requiere un número mínimo de celdas hexagonales a lo largo de la longitud antes de que cualquier promedio pueda considerarse único. Esto se traduce en que el parámetro $L / a_{1}>10$, donde $a_{1}$ es el ancho de la celda hexagonal y $L$ la longitud del CNT. Además, [34] parametriza la aplicabilidad del modelo viga de Navier y de la lámina delgada a partir de la respuesta a frente a pandeo por compresión, llegando a dominios de aplicación en función de los valores de ciertos parámetros clave.

Con una orientación similar, [107] utiliza el modelo viga junto con el modelo lámina en función de la esbeltez y desarrolla unos patrones de tensiones críticas en función de los números de onda, con el objetivo de determinar el modelo continuo óptimo en cada caso. Como cabía esperar, la influencia de la esbeltez resulta definitiva para la aplicabilidad del modelo viga a los CNTs.

Una modificación de los modelos viga que ajustan su aplicabilidad a los CNTs consiste en el modelo no local continuo [65], donde se adopta una nueva relación constitutiva donde la tensión depende de la deformación en un entorno volumétrico del punto considerado. De esta forma se introduce el efecto del detalle atómico como parámetros adicionales. Mediante el mismo tipo de modelo adaptado al cálculo dinámico, [57] estudia las vibraciones que produce el movimiento de nanopartículas en el interior de un SWNT.

\section{Tratamiento experimental}

Aunque el tratamiento experimental del problema no puede considerarse en sí mismo un modelo de análisis, se ha incluido como tal en el esquema de la figura 2.1 debido a su excepcional importancia. Un buen número de referencias [17], [50], [93], [99], [112], [118], [119], ya resumidas en esta sección, procesan los resultados obtenidos mediante el modelo viga de Navier, de ahí la relación adoptada en el esquema con dicho modelo.

Algunos estudios netamente experimentales [25], [27],[105] presentan evidencias cualitativas de la inestabilidad superficial (abolladura) que se produce al someter a MWNTs a flexión con grandes deformaciones mediante AFM o TEM. Iijima [43] también efectúa este tipo de ensayo, incorporando un análisis teórico mediante MD, empleando un potencial T-B y modelizando la interacción VDW a través del potencial L-J. Concluye que la formación de la abolladura a flexión se da a los $30^{\circ}$ de 
rotación entre los extremos en un tubo de $d=1.2 \mathrm{~nm}$, e insiste en la abolladura elástica sin rotura ni formación de defectos de $\mathrm{S}-\mathrm{W}$.

Otros estudios experimentales buscan analizar la interacción entre deformación mecánica y propiedades eléctricas [83], ensayando MWNTs a flexión con la punta de un AFM hasta la rotura y midiendo simultáneamente la resistencia eléctrica. Por otra parte, [38] analiza la deformabilidad transversal con la punta de un AFM sobre dos CNTs cruzados sobre un sustrato y efectúa un análisis teórico MD empleando un potencial armónico.

En [117] se puede consultar una amplia revisión de ensayos hasta la actualidad, y en [1] un particular registro de los ensayos orientados a composites, donde se afirma que la transferencia de carga eficaz entre los CNTs y la matriz polimérica depende principalmente en la mejora de interfaces nanotubo-matriz a través de la funcionalización química de los extremos de cada fragmento de tubo. Esta mejora permanece hoy por hoy como un reto en el mundo de los nanocomposites.

\subsubsection{El modelo pieza flexible 1D}

Como se ha podido comprobar en la sección 2.7.1, la mayoría de referencias consultadas que emplean un modelo viga 1D en el análisis, asumen la teoría clásica de Navier-Bernouilli con pequeños desplazamientos, limitándose así al rango lineal (o bien tratando no linealidades débiles [66], [108]).

Teniendo en cuenta la posible abolladura (observada en SWNTs) o el modo ondulado (más propio de MWNTs) de deformación, constatados experimentalmente, parece lógico que si pretendemos modelizar el comportamiento de los CNTs más allá del rango de las pequeñas deformaciones, debemos considerar la deformación de la sección transversal en su propio plano, requiriendo por tanto modelos pieza alargada tipo Cosserat. La energía de deformación de la pieza tendrá entonces un término adicional debido a las distorsiones y desplazamientos de los puntos de la sección en su propio plano [31].

Recientemente podemos encontrar trabajos que tratan la micro-estructura helicoidal de una pieza alargada integrada en un modelo de Cosserat no lineal de piezas flexibles ([36], [55] y [72]). Los autores citados proponen la aplicabilidad de este tipo de modelos a moléculas de ADN y a CNTs, aunque su aplicabilidad a la modelización de CNTs no está tratada con detalle.

En particular, Healey [36] propone una serie de definiciones de Hemitropía, Simetría transversal, Isotropía y Simetría helicoidal (Luo [72] toma otras definiciones mucho más complejas) muy relacionadas con el concepto de la objetividad expuesto en [56]. En general consisten en la invarianza de la energía de deformación respecto a ciertas transformaciones ortogonales de la pieza en sí misma (ya sean rotaciones, reflexiones o combinaciones de ambas). Lauderdale [55], propone algunas definiciones similares, pero para piezas con simetría helicoidal exclusivamente. Surge entonces la cuestión de si las tres simetrías helicoidales a $60^{\circ}$ entre sí que aparecen en la lámina de grafeno, son equivalentes a una isotropía o de si debemos incluir la quiralidad en el modelo viga para comprobar su influencia en el comportamiento estructural.

Por otra parte, teniendo en cuenta la incertidumbre en la determinación del espesor de pared para el cilindro equivalente a un cierto SWNT, es conveniente introducir las rigideces $K_{N}, K_{Q}, K_{M}$ y $K_{T}$ en la relación constitutiva del modelo como parámetros independientes [52],[92], sin necesidad de recurrir a las fórmulas clásicas $E A / L, G A^{*} / L, E I / L^{3}$ y $G J / L$. Esta idea nos proporciona una gran libertad para calibrar nuestro modelo con los datos experimentales.

\subsubsection{El modelo lámina}

El modelo lámina cilíndrica es un tipo de modelo continuo que proporciona de manera natural información del sistema real incapaz de ser captada por el modelo viga, tal como la deformación de la sección transversal del nanotubo. Para captar esta deformación, [91] simula fajos de SWNTs a compresión transversal hasta la abolladura mediante el modelo lámina, obteniendo 
$\sigma_{\text {cr,transv }}=0.73 \sigma_{c r, \text { long. }}$ Este resultado representa una buena muestra de la anisotropía de SWNTs como conjunto.

Al igual que en los modelos viga (Navier o tipo pieza flexible), una dificultad implícita en este tipo de modelos consiste en la elección de los parámetros de rigidez adecuados para reproducir el comportamiento real de CNTs frente a un sistema general de acciones. Por ejemplo, Huang [40] estudia el espesor equivalente de una lámina de grafeno por un tratamiento QC y afirma que tanto el espesor equivalente como el módulo de Young dependen del tipo de carga al que se someta a la lámina. Sin embargo, esta dependencia desaparece si consideramos las rigideces de placa como parámetros independientes entre sí. Análogamente [102] parametriza su modelo lámina a partir de rigideces a extensión, a flexión y a torsión independientes, a partir del análisis vibracional de un CNT simplemente apoyado en sus extremos.

Por otra parte, L. F. Wang [103] analiza (verificando los resultados mediante técnicas MD) la dependencia de las características elásticas y del espesor equivalente frente al diámetro del CNT, concluyendo que para los diámetros más pequeños se obtienen variaciones mayores. Por tanto, las características elásticas de un medio continuo en el modelo lámina para CNTs no son estrictamente constantes.

Algunos autores emplean el modelo lámina para el análisis de las inestabilidades locales. Q. Wang [107] estudia la abolladura para varios tamaños de CNTs y para diversos números de onda y concluye que la abolladura se produce para flexiones globales menores en nanotubos de mayor diámetro, [92] simula la respuesta MWNTs de doble capa a compresión para el análisis de la abolladura mediante un modelo lámina comparado con técnicas MD, y refuerza la idea de adoptar parámetros de rigidez independientes en el modelo lámina. Asimismo, [35] estudian la abolladura a compresión de MWNTs hasta con cuatro capas.

Para introducir el efecto del reducido tamaño de los CNTs en el modelo 2D continuo, Hu [39] propone un modelo lámina elástica no local. La microestructura del material, tal como el espaciado entre átomos, se convierte en determinante cuando el cuerpo es extremadamente reducido, de forma que la estructura discreta no se puede homogeneizar en un continuo. Los modelos clásicos de láminas no admiten dependencia intrínseca del tamaño al no incluir defectos. Sin embargo, el modelo no local supone que la tensión en un punto dado es función del campo de deformaciones en cada punto del cuerpo y en un entorno volumétrico del mismo. Por tanto, el efecto de escala se puede introducir en las ecuaciones constitutivas como un parámetro material.

Un fenómeno que puede distorsionar el estado de tensiones y deformaciones obtenido a partir de un modelo lámina es conocido como preenergía (ver sección 4.1.5), y consiste en la energía de deformación almacenada el CNT a partir de la lámina de grafeno plana (supuesta como origen de deformación), hasta su configuración inicial. Como se afirma en [103], Yakobson [115] modeliza un SWNT como una lámina grafítica delgada enrollada sin alargamiento, y calculan (mediante el potencial de T-B) la energía mínima $W_{0}$ (preenergía) relajando dicha lámina enrollada hasta su configuración optimizada y el exceso de energía $W_{1}$ cuando el SWNT es posteriormente sometido a una deformación axil $\epsilon$. La rigidez a axil de la lámina se puede computar entonces como la derivada segunda de $W_{1}$ con respecto a $\epsilon$ y la rigidez a flexión como la derivada segunda de $W_{0}$ con respecto a $\kappa$ (curvatura de la lámina).

\subsubsection{Método de los elementos finitos (MEF)}

Aunque en algunas referencias se emplean elementos finitos $2 \mathrm{D}$ como partes constituyentes auxiliares de un modelo más complejo [18], [81], en esta sección nos limitamos a la aplicación de los elementos finitos 3D (sólidos) como modelo fundamental de análisis en la simulación de CNTs. X. Wang [108] simula la vibración no lineal de MWNTs en ménsula mediante MEF, suponiendo cada nanotubo como un sólido continuo y reproduciendo el modo ondulado mediante elementos finitos 3D. A partir de los resultados obtenidos plantea un módulo de Young $E_{e f}$ en la forma de [66], 
convirtiendo una no linealidad geométrica en una no linealidad mecánica.

En general, los resultados de estos métodos coinciden bien con los observados experimentalmente, aunque la restricción computacional del orden de $10^{9}$ átomos presente en los análisis MD se mantiene también en el MEF, limitando su aplicabilidad actual a SWNTs o bien MWNTs de diámetros y longitudes reducidas.

Una variante al MEF capaz de reducir la discretización necesaria para reproducir resultados fiables es el Boundary Element Method (BEM) [68], aplicado al análisis de composites reforzados con CNTs. Se trata de un modelo continuo similar al FEM pero con una necesidad de discretización mucho menor y considerando el CNT dentro de un elemento representativo del material matriz. 



\section{Capítulo 3}

\section{Caracterización estática y cinemática del modelo MSM}

El objetivo de este capítulo es analizar la indeterminación estática y cinemática del modelo stickspiral adoptado en la investigación, adaptando a nuestro caso el método basado en la descomposición en valores singulares (SVD) desarrollada por Pellegrino en [84], [85] para estructuras articuladas. Se trata descartar posibles modos de mecanismo e identificar los modos de autotensión presentes en el sistema. De esta forma, estaremos en disposición de determinar la influencia de sus condiciones de sustentación y geometría en la existencia de soluciones al problema.

La justificación del presente análisis reside en la novedad que representa tratar el modelo stickspiral incluyendo un número indeterminado de barras y muelles. Además, la formulación de la tesis permite introducir cualquier sistema condiciones de vinculación y cargas externas, pudiendo producirse configuraciones estática o cinemáticamente indeterminadas.

\subsection{Descripción conceptual del modelo MSM stick-spiral}

Dada la naturaleza discreta de los SWNTs y teniendo en cuenta su geometría cilíndrica, adoptamos un modelo de mecánica estructural molecular (MSM) tipo stick-spiral (ver sección 2.4) basado en el paralelismo existente entre el campo de fuerzas interatómicas derivadas de cierto potencial molecular y un sistema de elementos estructurales coherentes con tales fuerzas.

Los nudos de este modelo representan átomos de C (supuestos puntuales), que se considerarán contenidos en una superficie cilíndrica. Su posición se determina mediante un mapeo conforme desde la lámina plana de grafeno a la superficie de un cilindro. Dicho mapeo conforme equivale a anclar los núcleos atómicos a un sistema de coordenadas convectivas sobre la lámina plana y posteriormente enrollarla formando un cilindro perfecto de diámetro $D_{t}$, determinado según la ecuación (1.1). En dicha ecuación se adopta $a_{0}=0.142 \mathrm{~nm}$, que es la distancia interatómica de equilibrio de un enlace covalente C-C en la lámina de grafeno.

Teniendo en cuenta la descripción de los potenciales interatómicos más habituales contenida en la sección 2.4.1, definiremos un elemento a axil tipo biela (o muelle longitudinal) entre cada dos átomos vecinos de $\mathrm{C}$ para reproducir la interacción longitudinal del enlace covalente C-C. Estos elementos estarán localizados en las secantes al cilindro y cada uno conectará una pareja de átomos de $\mathrm{C}$ adyacentes en la malla hexagonal. Por otra parte, la interacción de tres cuerpos consistente en la resistencia que oponen tres núcleos atómicos a que dos enlaces covalentes entre ellos modifiquen su orientación relativa, se plasma en el modelo mediante un elemento resorte angular localizado en el 
entorno inmediato de un nudo entre dos bielas contiguas. La definición del modelo para un $\mathrm{ZZ}(8,0)$ se ilustra en la figura 3.1 a modo de ejemplo.

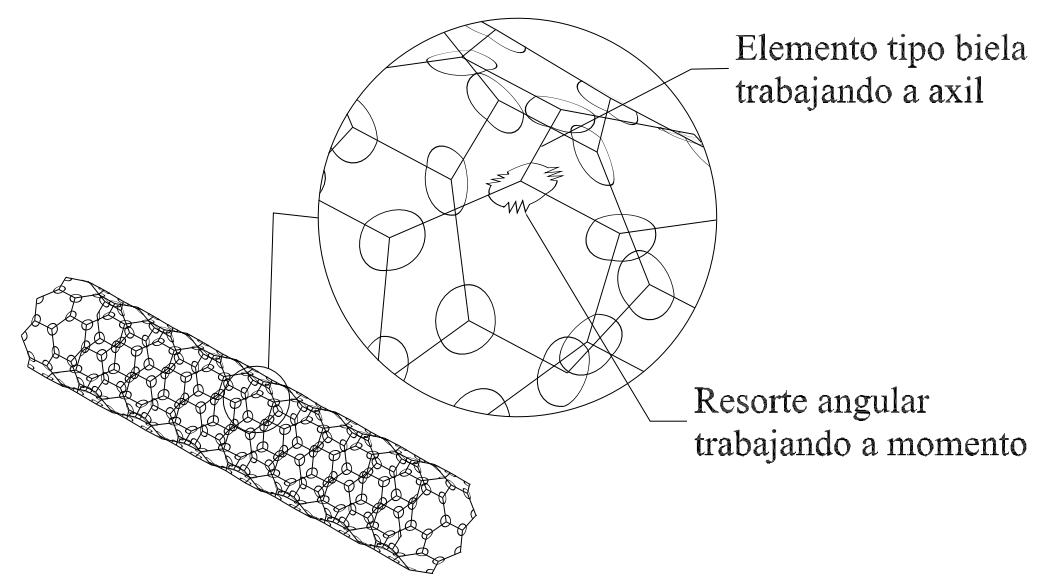

Figura 3.1: Modelización adoptada para un CNT Zig-Zag

\subsection{Revisión abreviada de la formulación SVD}

Como punto de partida para nuestra aplicación posterior, resumimos a continuación la caracterización estática y cinemática desarrollada en [84] y [85] para sistemas de barras articuladas. En dicho análisis se descompone en valores singulares (SVD) la matriz de equilibrio $\mathbf{H}$ de la estructura completa para averiguar el número de modos de mecanismo y de autotensión linealmente independientes.

La matriz $\mathbf{H}$ se obtiene ensamblando adecuadamente las matrices de equilibrio de las barras individuales, que contienen los vectores unitarios que describen la orientación de cada barra aislada. Del mismo modo, las matrices cinemáticas de la estructura completa y de cada barra son las traspuestas de las respectivas matrices de equilibrio, verificando las mismas propiedades de ensamblaje.

\subsubsection{Ecuaciones cinemáticas}

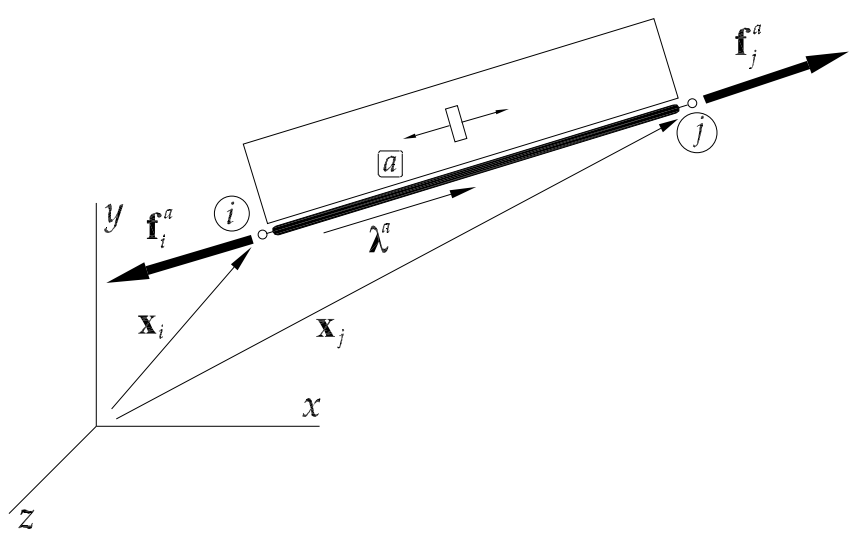

40

Figura 3.2: Definición vectores barra articulada 
Teniendo en cuenta los vectores representados en la figura 3.2 , podemos definir los siguientes conceptos:

1. Vector unitario de barra

$$
\lambda^{a}=\frac{\mathbf{x}_{j}-\mathbf{x}_{i}}{l^{a}}
$$

2. Longitud de la barra

$$
l^{a}=\sqrt{\left(\mathbf{x}_{j}-\mathbf{x}_{i}\right)^{T}\left(\mathbf{x}_{j}-\mathbf{x}_{i}\right)}
$$

3. Velocidades de los nudos $\quad \dot{\mathbf{x}}_{i}, \dot{\mathbf{x}}_{j}$

Derivando la longitud de la barra respecto al tiempo:

$$
\begin{aligned}
i^{a} & =\frac{1}{2 l^{a}}\left[\left(\dot{\mathbf{x}}_{j}-\dot{\mathbf{x}}_{i}\right)^{T}\left(\mathbf{x}_{j}-\mathbf{x}_{i}\right)+\left(\mathbf{x}_{j}-\mathbf{x}_{i}\right)^{T}\left(\dot{\mathbf{x}}_{j}-\dot{\mathbf{x}}_{i}\right)\right]= \\
& =\frac{1}{l^{a}}\left[\left(\mathbf{x}_{j}-\mathbf{x}_{i}\right)^{T}\left(\dot{\mathbf{x}}_{j}-\dot{\mathbf{x}}_{i}\right)\right]=\lambda^{a T}\left(\dot{\mathbf{x}}_{j}-\dot{\mathbf{x}}_{i}\right)
\end{aligned}
$$

matricialmente:

$$
l^{a}=\left[\begin{array}{ll}
-\lambda^{a T} & \lambda^{a T}
\end{array}\right]\left\{\begin{array}{c}
\dot{\mathbf{x}}_{i} \\
\dot{\mathbf{x}}_{j}
\end{array}\right\}
$$

Si tratamos los desplazamientos como diferenciales del vector de posición, podemos reescribir (3.4) como 1

$$
\mathbf{C}_{l}^{a} \cdot d \mathbf{x}^{a}=d l^{a} \longrightarrow \mathbf{C}_{l}^{a} \mathbf{u}^{a}=\epsilon^{a}
$$

donde:

$$
\begin{array}{ll}
\mathbf{C}_{l}^{a}=\left[\begin{array}{ll}
-\lambda^{a T} & \lambda^{a T}
\end{array}\right], & \text { Matriz cinemática de la barra aislada } \\
d \mathbf{x}^{a}=\mathbf{u}^{a}=\left\{\begin{array}{ll}
\mathbf{u}_{i} & \mathbf{u}_{j}
\end{array}\right\}^{T} & \text { Vector de desplazamientos nodales } \\
\epsilon^{a}=d l^{a} & \text { Alargamiento o deformación de barra. }
\end{array}
$$

\subsubsection{Ecuaciones de equilibrio}

Para el caso de piezas articuladas, resulta inmediato escribir las fuerzas en extremo de barra en función de los axiles de barra:

$$
\left\{\begin{array}{c}
\mathbf{f}_{i}^{a} \\
\mathbf{f}_{j}^{a}
\end{array}\right\}=n^{a}\left\{\begin{array}{c}
-\lambda^{a} \\
\lambda^{a}
\end{array}\right\}
$$

relación que se puede expresar de forma compacta como

$$
\mathbf{f}^{a}=n^{a} \mathbf{H}_{l}^{a}
$$

Estableciendo los equilibrios de nudo en cada conexión, es posible obtener la ecuación de equilibrio ensamblada para toda la estructura:

$$
\mathbf{H}_{l} \mathbf{n}=\overline{\mathbf{f}}
$$

donde:

$\mathbf{H}_{l} \quad$ Matriz de equilibrio ensamblada (ver nota 1 al pie)

n Vector de axiles de barra

$\overline{\mathbf{f}} \quad$ Vector de fuerzas nodales externas.

\footnotetext{
${ }^{1}$ Se ha denotado con ()$_{l}$ a las variables relacionadas con un análisis geométricamente lineal (valores en la geometría inicial) para diferenciarlas de las correspondientes al análisis no lineal geométrico, que se desarrollará en la sección 5.1
} 


\subsubsection{Relación de contragradiencia}

Aplicando la ecuación de los trabajos virtuales (ETV) a la estructura completa:

$$
\overline{\mathbf{f}} \cdot \delta \mathbf{u}=\mathbf{n} \cdot \delta \boldsymbol{\epsilon} \quad \forall \delta \mathbf{u}
$$

Mediante el empleo de las ecuaciones (3.5) y 3.8

$$
\begin{array}{r}
\mathbf{H}_{l} \mathbf{n} \cdot \delta \mathbf{u}=\mathbf{n} \cdot \mathbf{C}_{l} \delta \mathbf{u} \\
\mathbf{n}^{T} \mathbf{H}_{l}^{T} \delta \mathbf{u}=\mathbf{n}^{T} \mathbf{C}_{l} \delta \mathbf{u}
\end{array}
$$

Por tanto

$$
\mathbf{C}_{l}=\mathbf{H}_{l}^{T}
$$

conocida como relación de contragradiencia.

\subsubsection{Descomposición en valores singulares (SVD) de la matriz de equilibrio}

Sea $\mathbf{H}_{l}$ la matriz de equilibrio de la estructura completa de tamaño $n \times m$ y rango $r$ (número de vectores linealmente independientes en la matriz), entonces $\mathbf{H}_{l}$ admite una descomposición en valores singulares (SVD) del siguiente modo:

$$
\mathbf{H}_{l}=\mathbf{U V W} \mathbf{W}^{T}
$$

donde $\mathbf{U}$ es una matriz ortogonal de orden $n, \mathbf{W}$ es una matriz ortogonal de orden $m$ y $\mathbf{V}$ es una matriz diagonal de orden $n \times m$ con $r$ elementos positivos $v_{i}$ en la diagonal (valores singulares) y el resto nulos. Por tanto:

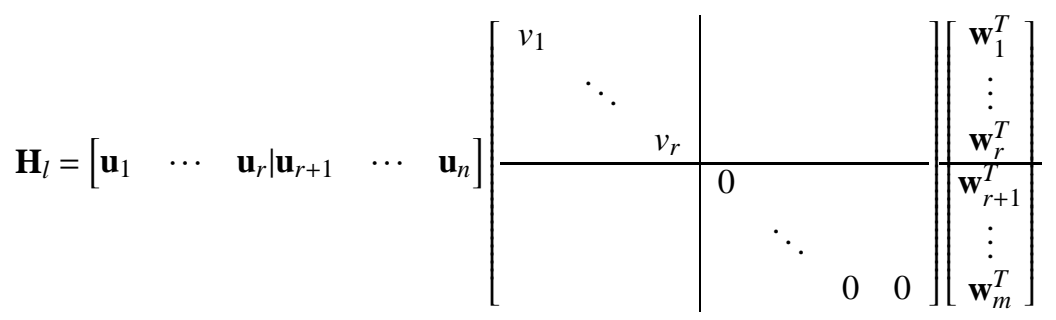

Puesto que $\mathbf{W}$ es ortogonal, postmultiplicando (3.12) por $\mathbf{W}$ y expresando las matrices $\mathbf{U}, \mathbf{W}$ como un conjunto de vectores $\mathbf{u}_{i}, \mathbf{w}_{i}$ respectivamente, se verificará:

$$
\begin{array}{lr}
\mathbf{H}_{l} \mathbf{w}_{i}=v_{i} \mathbf{u}_{i} & 1 \leq i \leq r \\
\mathbf{H}_{l} \mathbf{w}_{i}=\mathbf{0} & r+1 \leq i \leq m
\end{array}
$$

La forma de las ecuaciones (3.14) comparada con (3.8) muestra que los vectores $\mathbf{w}_{i}$ representan $r$ sistemas de axiles estáticamente admisibles y en equilibrio con los $n$ sistemas de fuerzas exteriores $v_{i} \mathbf{u}_{i}$, respectivamente. Análogamente, los vectores $\left[\mathbf{w}_{r+1} \cdots \mathbf{w}_{m}\right]$ describen los $q=m-r$ modos de autotensión de la estructura (sistemas de axiles en equilibrio con sistemas nulos de fuerzas exteriores). Por tanto $q$ será el grado de indeterminación estática de la estructura.

Trasponiendo la matriz de equilibrio $\mathbf{C}_{l}=\mathbf{H}_{l}^{T}=\mathbf{W} \mathbf{V}^{T} \mathbf{U}^{T}$, y operando de la misma forma se obtiene:

$$
\begin{array}{lr}
\mathbf{C}_{l} \mathbf{u}_{i}=v_{i} \mathbf{w}_{i} & 1 \leq i \leq r \\
\mathbf{C}_{l} \mathbf{u}_{i}=\mathbf{0} & r+1 \leq i \leq n
\end{array}
$$


De manera dual, los vectores $\mathbf{u}_{i}$ son $r$ sistemas de desplazamientos nodales compatibles y consistentes con los $r$ modos de deformación de la estructura $v_{i} \mathbf{w}_{i}$, respectivamente. Los vectores $\left[\mathbf{u}_{r+1} \cdots \mathbf{u}_{n}\right.$ ] describen los $p=n-r$ posibles modos de mecanismo en la estructura (modos de desplazamiento compatibles con sistemas nulos de deformaciones en las barras de la estructura). Por tanto $p$ es el grado de indeterminación cinemática o grado de mecanismo de la estructura.

Podemos deducir las siguientes conclusiones:

1. Los vectores asociados a los $r$ valores singulares representan:

a) Los $r$ sistemas de fuerzas exteriores $v_{i} \mathbf{u}_{i}$ en equilibrio con $r$ conjuntos de esfuerzos axiles $\mathbf{w}_{i}$, o de modo dual,

b) Los $r$ sistemas de desplazamientos nodales $\mathbf{u}_{i}$ compatibles con los $r$ conjuntos de alargamientos $v_{i} \mathbf{w}_{i}$.

2. Los q vectores $\left[\mathbf{w}_{r+1} \cdots \mathbf{w}_{m}\right.$ ] son los modos de autotensión, pero también se pueden interpretar como los modos incompatibles de deformación.

3. Los $\mathrm{p}$ vectores $\left[\mathbf{u}_{r+1} \cdots \mathbf{u}_{n}\right]$ son los modos de mecanismo, y también representan los modos de carga de la estructura que no pueden ser equilibrados.

\subsection{Aplicación de la descomposición en valores singulares al mo- delo MSM}

\subsubsection{Ecuaciones cinemáticas incluyendo distorsiones angulares}

Admitiremos como válidas las ecuaciones cinemáticas (3.5) para los elementos barra del modelo stick-spiral, debiendo deducir una expresión análoga para los elementos muelle que nos permita introducir las distorsiones angulares en el análisis. Un esquema del resorte angular genérico viene representado en la figura 3.3 .

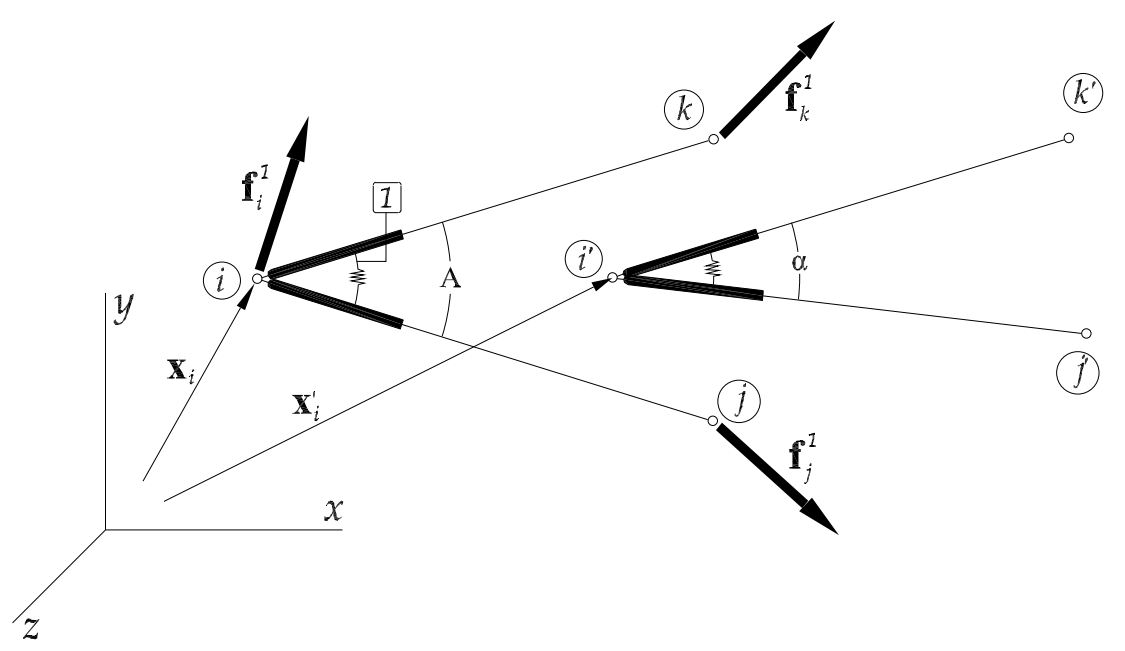

Figura 3.3: Resorte angular genérico 
Comentario 3.3.1 En la figura 3.3 se pretende representar el entorno inmediato del nudo $i$ conteniendo el resorte angular que afecta a las barras a (entre los nudos $i, j$ ) y b (entre los nudos $i, k$ ). En esta línea, se ha denotado por $\mathbf{f}_{i}^{1}, \mathbf{f}_{j}^{1}, \mathbf{f}_{k}^{1}$ a la fracción de las acciones nodales externas que contribuyen a la distorsión angular del resorte angular (en contraste con la figura 3.4 donde se han representado las acciones externas totales sobre la unidad básica).

Véase también que hemos empleado superíndices alfabéticos $\alpha=a, b, c, \ldots$ para los elementos barra, y numéricos $\beta=1,2,3, \ldots$ para los elementos muelle.

La deformación asociada a cada resorte angular será la distorsión angular, definida como:

$$
\epsilon^{1}=d \gamma^{1}=A-\alpha
$$

de donde se puede deducir directamente el criterio de signos adoptado, es decir, $\epsilon^{1}=d \gamma^{1}$ será positiva si el ángulo formado por ambas barras se cierra en el proceso de deformación.

Análogamente a las ecuaciones cinemáticas (3.5), buscamos una expresión para el resorte angular del tipo:

$$
\begin{gathered}
{\left[\begin{array}{ccc}
\boldsymbol{\Gamma}_{j}^{1^{T}} & \boldsymbol{\Gamma}_{i}^{1^{T}} & \boldsymbol{\Gamma}_{k}^{1^{T}}
\end{array}\right]\left\{\begin{array}{l}
\mathbf{u}_{j} \\
\mathbf{u}_{i} \\
\mathbf{u}_{k}
\end{array}\right\}=\left\{\epsilon^{1}\right\}} \\
\mathbf{C}_{l}^{1} \mathbf{u}^{1}=\epsilon^{1}
\end{gathered}
$$

Nótese que aunque $\epsilon^{1}$ es una deformación angular, el vector de desplazamientos nodales $\left\{\mathbf{u}_{j} \mathbf{u}_{i} \mathbf{u}_{k}\right\}^{T}$ sólo incluye componentes de traslación, es decir, las posibles rotaciones de los nudos no se consideran como variables cinemáticas del modelo.

Trataremos de determinar a continuación las tres funciones vectoriales $\boldsymbol{\Gamma}_{j}^{1}, \boldsymbol{\Gamma}_{i}^{1}, \boldsymbol{\Gamma}_{k}^{1}$ introducidas en la ecuación (3.17). Para este propósito, es suficiente emplear un cálculo geométricamente lineal y se admite la hipótesis de pequeños desplazamientos y pequeñas deformaciones. Tomando cosenos en (3.16) y simplificando

$$
\begin{gathered}
\cos \alpha=\cos \left(A-d \gamma^{1}\right)=\cos A \cos d \gamma^{1}+\sin d \gamma^{1} \sin A \simeq \cos A+d \gamma^{1} \sin A \\
\epsilon^{1}=d \gamma^{1}=\frac{\cos \alpha-\cos A}{\sin A}
\end{gathered}
$$

donde podemos calcular cada razón trigonométrica como:

$$
\begin{aligned}
& \cos A=\lambda^{a T} \lambda^{b}=\frac{\left(\mathbf{x}_{j}-\mathbf{x}_{i}\right)^{T}\left(\mathbf{x}_{k}-\mathbf{x}_{i}\right)}{l^{a} l^{b}} \\
& \sin A=\sqrt{1-\cos ^{2} A} \\
& \cos \alpha=\frac{\left[\mathbf{x}_{j}+\mathbf{u}_{j}-\left(\mathbf{x}_{i}+\mathbf{u}_{i}\right)\right]^{T}\left[\mathbf{x}_{k}+\mathbf{u}_{k}-\left(\mathbf{x}_{i}+\mathbf{u}_{i}\right)\right]}{l^{a^{\prime}} l^{b^{\prime}}}=\frac{C_{N}}{C_{D}}
\end{aligned}
$$

desarrollamos el numerador de (3.19c) según:

$$
\begin{aligned}
C_{N}=[\overbrace{\mathbf{x}_{j}-\mathbf{x}_{i}}^{l^{a} \lambda^{a}} & \left.+\left(\mathbf{u}_{j}-\mathbf{u}_{i}\right)\right]^{T}[\overbrace{\mathbf{x}_{k}-\mathbf{x}_{i}}^{l^{b} \lambda^{b}}+\left(\mathbf{u}_{k}-\mathbf{u}_{i}\right)]= \\
& =l^{a} l^{b} \lambda^{a T} \lambda^{b}+l^{a} \lambda^{a T}\left(\mathbf{u}_{k}-\mathbf{u}_{i}\right)+l^{b} \lambda^{b^{T}}\left(\mathbf{u}_{j}-\mathbf{u}_{i}\right)+O\left(\varepsilon^{2}\right)
\end{aligned}
$$

y el denominador según:

$$
C_{D}=\left(l^{a}+\epsilon^{a}\right)\left(l^{b}+\epsilon^{b}\right)=l^{a} l^{b}+l^{a} \epsilon^{b}+l^{b} \epsilon^{a}+O\left(\varepsilon^{2}\right)
$$



queda:

despreciando los términos de segundo orden en (3.20), (3.21) y sustituyendo, la expresión (3.19c)

$$
\cos \alpha=\frac{l^{a} l^{b} \lambda^{a T} \lambda^{b}+l^{a} \lambda^{a T}\left(\mathbf{u}_{k}-\mathbf{u}_{i}\right)+l^{b} \lambda^{b^{T}}\left(\mathbf{u}_{j}-\mathbf{u}_{i}\right)}{l^{a} l^{b}+l^{a} \epsilon^{b}+l^{b} \epsilon^{a}}
$$

Sustituyendo ahora (3.22) y (3.19a) en 3.18), obtenemos:

$$
\epsilon^{1}=\frac{1}{\sin A}\left\{\frac{\cos A+\frac{\lambda^{a T}}{l^{b}}\left(\mathbf{u}_{k}-\mathbf{u}_{i}\right)+\frac{\lambda^{b^{T}}}{l^{a}}\left(\mathbf{u}_{j}-\mathbf{u}_{i}\right)-\left(1+\epsilon^{a}+\epsilon^{b}\right) \cos A}{1+\epsilon^{a}+\epsilon^{b}}\right\}
$$

Empleando las ecuaciones cinemáticas (3.5) para ambas barras y despreciando los términos de deformación en el denominador de (3.23), llegamos a:

$$
\begin{aligned}
\epsilon^{1} & =\left[\frac{-1}{\sin A}\left(\frac{\lambda^{a T}}{l^{b}}+\frac{\lambda^{b^{T}}}{l^{a}}\right)+\frac{1}{\tan A}\left(\frac{\lambda^{a T}}{l^{a}}+\frac{\lambda^{b^{T}}}{l^{b}}\right)\right] \mathbf{u}_{i}+\left[\frac{1}{\sin A} \frac{\lambda^{b^{T}}}{l^{a}}-\frac{1}{\tan A} \frac{\lambda^{a T}}{l^{a}}\right] \mathbf{u}_{j}+ \\
& +\left[\frac{1}{\sin A} \frac{\lambda^{a T}}{l^{b}}-\frac{1}{\tan A} \frac{\lambda^{b^{T}}}{l^{b}}\right] \mathbf{u}_{k}=\boldsymbol{\Gamma}_{i}^{1^{T}} \mathbf{u}_{i}+\Gamma_{j}^{1^{T}} \mathbf{u}_{j}+\boldsymbol{\Gamma}_{k}^{1^{T}} \mathbf{u}_{k}
\end{aligned}
$$

Por tanto, las funciones vectoriales $\boldsymbol{\Gamma}$ adoptarán la expresión:

$$
\begin{aligned}
\boldsymbol{\Gamma}_{j}^{1} & =\frac{1}{\sin A} \frac{\lambda^{b}}{l^{a}}-\frac{1}{\tan A} \frac{\lambda^{a}}{l^{a}} \\
\boldsymbol{\Gamma}_{k}^{1} & =\frac{1}{\sin A} \frac{\lambda^{a}}{l^{b}}-\frac{1}{\tan A} \frac{\lambda^{b}}{l^{b}} \\
\boldsymbol{\Gamma}_{i}^{1} & =-\boldsymbol{\Gamma}_{j}^{1}-\boldsymbol{\Gamma}_{k}^{1}
\end{aligned}
$$

Consideremos ahora una unidad básica formada por dos elementos tipo biela unidos mediante un único resorte angular en su configuración no deformada, tal como se representa en la figura 3.4.

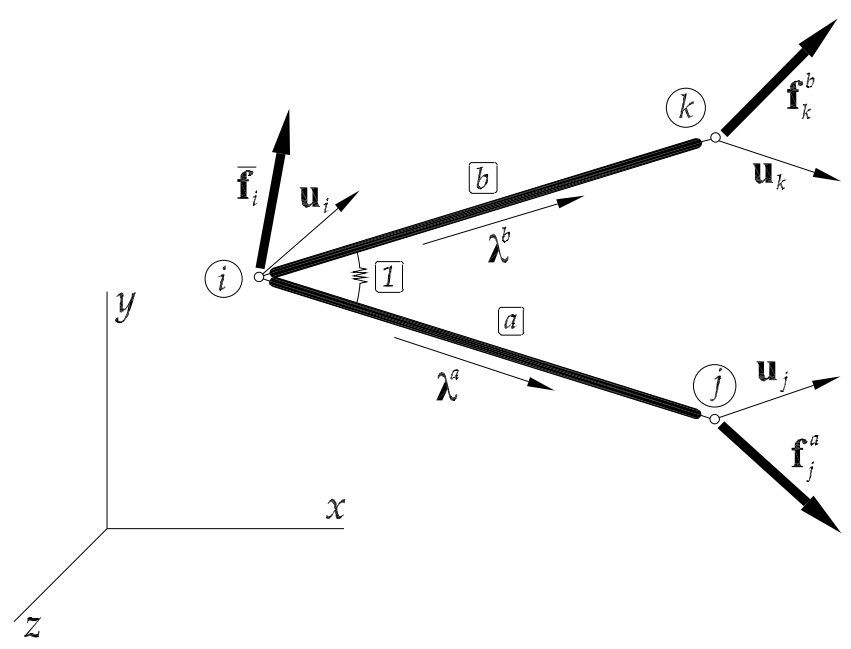

Figura 3.4: Vectores unidad básica (Configuración Inicial)

Agrupando las ecuaciones cinemáticas (3.5) y 3.17) para cada elemento constituyente, es intuitivo llegar a la siguiente expresión de las ecuaciones cinemáticas ensambladas del sistema elemental: 


$$
\left[\begin{array}{ccc}
\lambda^{a T} & -\lambda^{a T} & \mathbf{0} \\
\mathbf{0} & -\lambda^{b^{T}} & \lambda^{b^{T}} \\
\boldsymbol{\Gamma}_{j}^{1^{T}} & \boldsymbol{\Gamma}_{i}^{1^{T}} & \boldsymbol{\Gamma}_{k}^{1}
\end{array}\right]\left\{\begin{array}{c}
\mathbf{u}_{j} \\
\mathbf{u}_{i} \\
\mathbf{u}_{k}
\end{array}\right\}=\left\{\begin{array}{c}
\epsilon^{a} \\
\epsilon^{b} \\
\epsilon^{1}
\end{array}\right\}
$$

Asimismo, la ecuación (3.26a) se puede extender a cualquier sistema de bielas y resortes angulares correctamente ensamblados, sin más que introducir los vectores $\lambda$ y $\boldsymbol{\Gamma}$ necesarios en la matriz $\mathbf{C}_{l}$ :

$$
\mathbf{C}_{l} \mathbf{u}=\boldsymbol{\epsilon}
$$

Además, la ecuación cinemática general así construida se podrá descomponer en bloques según:

$$
\left[\begin{array}{l}
\mathbf{C}^{\lambda} \\
\mathbf{C}^{\Gamma}
\end{array}\right]\{\mathbf{u}\}=\left\{\begin{array}{l}
\boldsymbol{\epsilon}^{\lambda} \\
\boldsymbol{\epsilon}^{\Gamma}
\end{array}\right\}
$$

donde:

$\boldsymbol{\epsilon}^{\lambda} \quad$ Vector de deformaciones longitudinales de barra

$\boldsymbol{\epsilon}^{\Gamma} \quad$ Vector de distorsiones angulares de muelle

$\mathbf{C}^{\lambda}$ Matriz cinemática del sistema correspondiente a las deformaciones longitudinales

$\mathbf{C}^{\Gamma} \quad$ Matriz cinemática del sistema correspondiente a las distorsiones angulares

u Campo de desplazamientos nodales

Comentario 3.3.2 En un sistema ensamblado general de bielas y resortes, las orientaciones de los vectores unitarios de barra viene predefinida y no siempre serán salientes del elemento muelle a considerar.

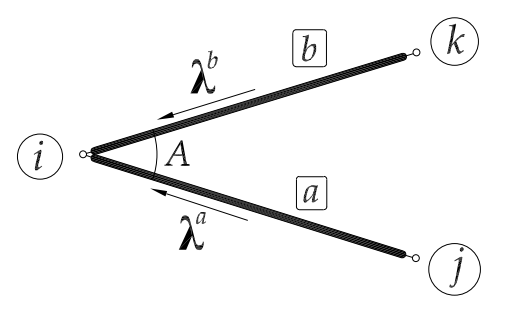

(a) Conexión frontal-frontal

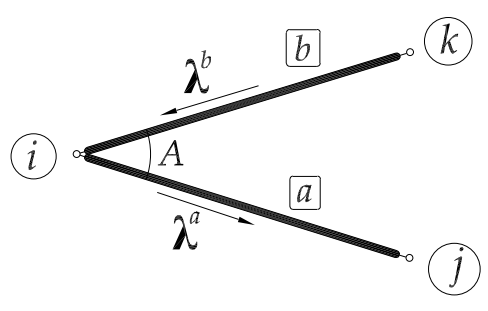

(b) Conexión dorsal-frontal

Figura 3.5: Esquema de orientaciones de barra

En consecuencia, las expresiones (3.25) deberán modificarse en su forma más general según:

$$
\begin{aligned}
\Gamma_{j}^{1} & =\frac{s^{b} \lambda^{b}}{l^{a} \sin A}-\frac{s^{a} \lambda^{a}}{l^{a} \tan A} \\
\boldsymbol{\Gamma}_{k}^{1} & =\frac{s^{a} \lambda^{a}}{l^{b} \sin A}-\frac{s^{b} \lambda^{b}}{l^{b} \tan A} \\
\boldsymbol{\Gamma}_{i}^{1} & =-\boldsymbol{\Gamma}_{j}^{1}-\boldsymbol{\Gamma}_{k}^{1}
\end{aligned}
$$

donde $s^{a}, s^{b}=1$ si el correspondiente unitario es saliente y $s^{a}, s^{b}=-1$ si es entrante. Por ejemplo, en la figura 3.5(a) adoptan unos valores $s^{a}=s^{b}=-1$ y en la 3.5(b) serán $s^{a}=1, s^{b}=-1$.

Por otra parte, el ángulo A siempre será el contenido entre ambas bielas y se deberá evaluar a partir de los unitarios de barra como

$$
\cos A=s^{a} \lambda^{a} \cdot s^{b} \lambda^{b}
$$

con los valores de $s^{a}, s^{b}$ aquí definidos. 


\subsubsection{Ecuaciones de equilibrio incluyendo resortes angulares}

Admitiremos que la ecuación de equilibrio de barra (3.7) es aplicable para las bielas del modelo stick-spiral y desarrollaremos unas expresiones análogas para los elementos muelle, extendiendo los resultados para cualquier sistema de estos elementos correctamente ensamblados.

\section{Elemento muelle}

Aplicando la ecuación de trabajos virtuales (ETV) al elemento muelle aislado (ver figura 3.3), resulta inmediato obtener su ecuación de equilibrio a través de la relación de contragradiencia expresada en (3.11). Esto es, supongamos que la ecuación de equilibrio del resorte angular admite una expresión del tipo:

$$
\mathbf{f}^{1}=m^{1} \mathbf{H}_{l}^{1}
$$

donde:

$$
\begin{array}{ll}
\mathbf{f}^{1}=\left\{\begin{array}{lll}
\mathbf{f}_{j}^{1} & \mathbf{f}_{i}^{1} & \mathbf{f}_{k}^{1}
\end{array}\right\}^{T} & \begin{array}{l}
\text { Fracción de las fuerzas externas que } \\
\text { colaboran a la distorsión angular del resorte }
\end{array} \\
m^{1} & \text { Momento en el resorte angular, positivo si tiende a cerrarlo } \\
\mathbf{H}_{l}^{1} & \text { Matriz de equilibrio del resorte (aún por determinar) }
\end{array}
$$

entonces la ETV se puede enunciar:

$$
\mathbf{f}^{1^{T}} \delta \mathbf{u}^{1}=m^{1} \delta \epsilon^{1}
$$

empleando (3.17) y 3.28, deducimos:

$$
m^{1} \mathbf{H}_{l}^{1^{T}} \delta \mathbf{u}^{1}=m^{1} \mathbf{C}_{l}^{1} \delta \mathbf{u}^{1}
$$

Por tanto

$$
\mathbf{H}_{l}^{1}=\mathbf{C}_{l}^{1^{T}}
$$

Esta última relación de contragradiencia nos permite verificar (3.28) si y sólo si la matriz de equilibrio $\mathbf{H}_{l}^{1}$ coincide con la traspuesta de la matriz cinemática $\mathbf{C}_{l}^{1}$.

\section{Unidad básica}

Considerando la unidad básica representada en la figura 3.4 y aplicando la ETV:

$$
\mathbf{f}_{j}^{a T} \delta \mathbf{u}_{j}+\overline{\mathbf{f}}_{i}^{T} \delta \mathbf{u}_{i}+\mathbf{f}_{k}^{b^{T}} \delta \mathbf{u}_{k}=n^{a} \delta \epsilon^{a}+n^{b} \delta \epsilon^{b}+m^{1} \delta \epsilon^{1}
$$

Tomando primeras variaciones en las ecuaciones cinemáticas (3.5) y (3.17), y sustituyendo:

$$
\begin{aligned}
& {\left[\begin{array}{lll}
\mathbf{f}_{j}^{a T} & \overline{\mathbf{f}}_{i}^{T} & \mathbf{f}_{k}^{b^{T}}
\end{array}\right]\left\{\begin{array}{l}
\delta \mathbf{u}_{j} \\
\delta \mathbf{u}_{i} \\
\delta \mathbf{u}_{k}
\end{array}\right\}=n^{a}\left[\begin{array}{ll}
-\lambda^{a T} & \lambda^{a T}
\end{array}\right]\left\{\begin{array}{l}
\delta \mathbf{u}_{i} \\
\delta \mathbf{u}_{j}
\end{array}\right\}+} \\
& +n^{b}\left[\begin{array}{ll}
-\lambda^{b^{T}} & \lambda^{b^{T}}
\end{array}\right]\left\{\begin{array}{l}
\delta \mathbf{u}_{i} \\
\delta \mathbf{u}_{k}
\end{array}\right\}+m^{1}\left[\begin{array}{lll}
\boldsymbol{\Gamma}_{j}^{1} & \boldsymbol{\Gamma}_{i}^{1^{T}} & \boldsymbol{\Gamma}_{k}^{1 T}
\end{array}\right]\left\{\begin{array}{l}
\delta \mathbf{u}_{j} \\
\delta \mathbf{u}_{i} \\
\delta \mathbf{u}_{k}
\end{array}\right\}
\end{aligned}
$$

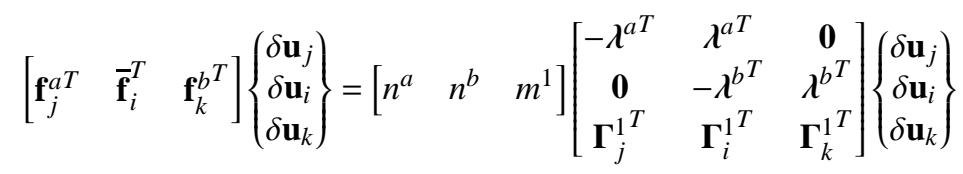


Agrupando:

$$
\overline{\mathbf{f}}^{T} \delta \mathbf{u}=\mathbf{p}^{T} \mathbf{C}_{l} \delta \mathbf{u}
$$

luego

$$
\overline{\mathbf{f}}=\mathbf{C}_{l}^{T} \mathbf{p}=\mathbf{H}_{l} \mathbf{p}
$$

donde:

f Vector de fuerzas externas al sistema considerado

$\mathbf{H}_{l} \quad$ Matriz de equilibrio del sistema considerado

$\mathbf{p}=\{\mathbf{n}, \mathbf{m}\}^{T} \quad$ Vector de esfuerzos internos ordenados en axiles $\mathbf{n}$ y momentos $\mathbf{m}$

Por tanto, la relación de contragradiencia $\mathbf{H}_{l}=\mathbf{C}_{l}^{T}$ también se cumple para la unidad básica, de forma que la matriz de equilibrio se ensambla de un modo idéntico a la matriz cinemática incluida en la expresión (3.26a) sin más que disponer los unitarios de barra y funciones $\boldsymbol{\Gamma}$ de muelle como cofactores de los desplazamientos de nudo correspondientes.

Comentario 3.3.3 Se debe hacer notar que aunque las fuerzas externas a la unidad básica $\overline{\mathbf{f}}=$ $\left\{\mathbf{f}_{j}^{a}, \overline{\mathbf{f}}_{i}, \mathbf{f}_{k}^{b}\right\}^{T}$ son los cofactores de los desplazamientos nodales $\delta \mathbf{u}$, sólo las $\overline{\mathbf{f}}_{i}$ se definen estrictamente como fuerzas externas, ya que aún siendo $\mathbf{f}_{j}^{a}$, $\mathbf{f}_{k}^{b}$ fuerzas externas a la unidad básica, serían consideradas de extremo de barra en un sistema estructural más complejo.

\section{Sistema estructural completo}

Para cualquier sistema estructural formado a partir de bielas y resortes angulares como los definidos en este capítulo, es inmediato demostrar la relación de contragradiencia. Además, la relación de equilibrio 3.35) se puede extender a dicho sistema, que descompuesta en bloques correspondientes a unitarios y funciones $\boldsymbol{\Gamma}$ se puede escribir:

$$
\overline{\mathbf{f}}=\left[\begin{array}{ll}
\mathbf{H}^{\lambda} & \mathbf{H}^{\Gamma}
\end{array}\right]\left\{\begin{array}{l}
\mathbf{n} \\
\mathbf{m}
\end{array}\right\}=\mathbf{H}^{\lambda} \mathbf{n}+\mathbf{H}^{\Gamma} \mathbf{m}
$$

donde:

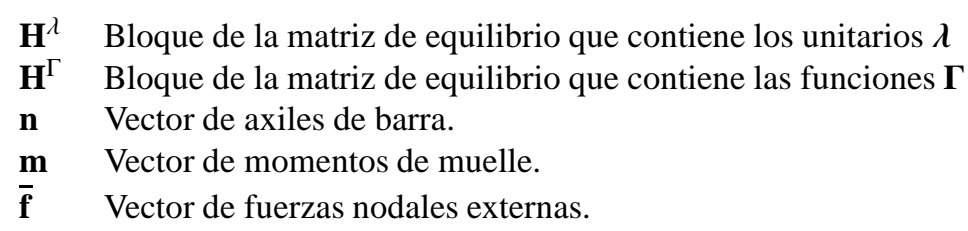

\subsubsection{Aplicación de las condiciones de contorno}

Respecto al sistema estructural completo, se trata en esta sección de introducir en la formulación las condiciones de vinculación externas. Como puede deducirse de los desarrollos anteriores, sólo se podrán introducir apoyos articulados que impidan uno o varios de los desplazamientos lineales en los nudos donde se aplican.

Consideremos la ecuaciones cinemáticas del sistema completo en la forma (3.26b). Para cualquier sistema general de coacciones, siempre seremos capaces de reordenar (permutando columnas) la matriz cinemática $\mathbf{C}_{l}$ y el campo de desplazamientos $\mathbf{u}$ (permutando componentes) hasta llegar a una descomposición por bloques del tipo:

$$
\left[\begin{array}{ll}
\mathbf{C}_{R} & \mathbf{C}_{A}
\end{array}\right]\left\{\begin{array}{l}
\mathbf{u}_{R} \\
\mathbf{u}_{A}
\end{array}\right\}=\boldsymbol{\epsilon}
$$


donde:

$\mathbf{C}_{R} \quad$ Bloque de la matriz cinemática asociada a desplazamientos libres

$\mathbf{C}_{A} \quad$ Bloque de la matriz cinemática asociada a desplazamientos coaccionados

$\mathbf{u}_{R} \quad$ Componentes de desplazamiento libres

$\mathbf{u}_{A} \quad$ Componentes de desplazamiento coaccionados

Puesto que para el análisis SVD es suficiente introducir apoyos fijos descartando los desplazamientos impuestos, se verifica que $\mathbf{u}_{A}=\mathbf{0}$, quedando una expresión reducida de las ecuaciones cinemáticas:

$$
\mathbf{C}_{R} \mathbf{u}_{R}=\boldsymbol{\epsilon}
$$

Razonando idénticamente sobre las ecuaciones de equilibrio del sistema completo (3.36), podemos encontrar una descomposición de $\mathbf{H}_{l}$ (permutando filas) del tipo:

$$
\left\{\begin{array}{l}
\overline{\mathbf{f}}_{R} \\
\overline{\mathbf{f}}_{A}
\end{array}\right\}=\left[\begin{array}{l}
\mathbf{H}_{R} \\
\mathbf{H}_{A}
\end{array}\right] \mathbf{p}
$$

donde:

$\mathbf{H}_{R} \quad$ Bloque de la matriz de equilibrio asociada a desplazamientos libres

$\mathbf{H}_{A} \quad$ Bloque de la matriz de equilibrio asociada a desplazamientos coaccionados

$\overline{\mathbf{f}}_{R} \quad$ Cargas nodales sobre los g.d.l. permitidos

$\overline{\mathbf{f}}_{A} \quad$ Fuerzas reacción asociadas a los g.d.l. coaccionados

Teniendo en cuenta que $\overline{\mathbf{f}}_{A}$ es un vector de componentes desconocidas, trabajaremos con la relación de equilibrio reducida:

$$
\overline{\mathbf{f}}_{R}=\mathbf{H}_{R} \mathbf{p}
$$

Por supuesto, es inmediato demostrar la relación de contragradiencia $\mathbf{H}_{R}=\mathbf{C}_{R}^{T}$ partiendo de la ETV. De hecho, son estas dos matrices sobre las que se efectúa la descomposición SVD descrita en la sección 3.2.4 para el análisis de la determinación estática y cinemática de SWNTs.

\subsection{Resultados y discusión}

Los resultados numéricos y gráficos del análisis se han obtenido mediante códigos propios. Para la generación de la geometría de SWNTs y la salida de modos de autotensión y de mecanismo se ha empleado VisualLISP [98] con el objetivo de aprovechar la potencia visual de AutoCAD ${ }^{\circledR}$. En cambio, el núcleo de cálculo se desarrolló en MATLAB ${ }^{\circledR}$, ya que la descomposición en Valores Singulares viene implementada automáticamente como una función interna del lenguaje.

Como resultados se obtienen el número de modos de autotensión y/o de mecanismo, los esfuerzos y reacciones de cada modo de autotensión y un movimiento representativo cinemáticamente admisible para cada modo de mecanismo. Como ejemplo, representamos la figura 3.6la geometría y apoyos introducidos para un nanotubo $\mathrm{AC}(3,3)$ con $n_{L}=4,5$ celdas en la dirección longitudinal y un único apoyo fijo en el nudo 1.

Se obtienen los tres modos de mecanismo trazados en la figura 3.7

y representamos como ejemplo las salidas de esfuerzos para los modos de autotensión 1, 35 y 66 en la figura 3.8. Se obtuvieron 66 modos de autotensión linealmente independientes.

También incluimos en la tabla 3.1 los resultados para varios SWNTs. Se denota por $n_{L}$ el número de celdas longitudinales para los nanotubos $\operatorname{Armchair}(\mathrm{AC})$ y $\operatorname{ZigZag}(\mathrm{ZZ})$, y por $n$-mod el número de veces que se dispone el módulo del vector traslación (definido en el capítulo 1) en la dirección longitudinal hasta alcanzar la longitud total para los Chiral $(\mathrm{CH})$. En la columna de descripción de las 


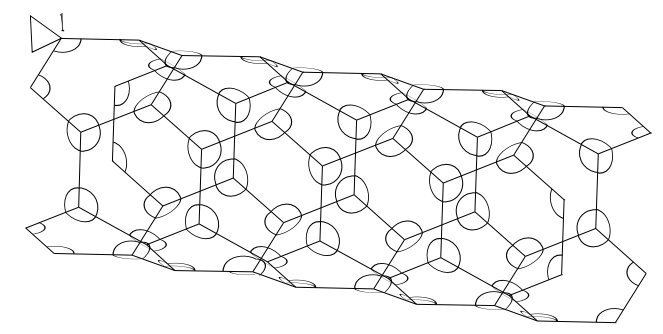

Figura 3.6: Geometría y apoyos $A C(3,3), n_{L}=4.5$ celdas
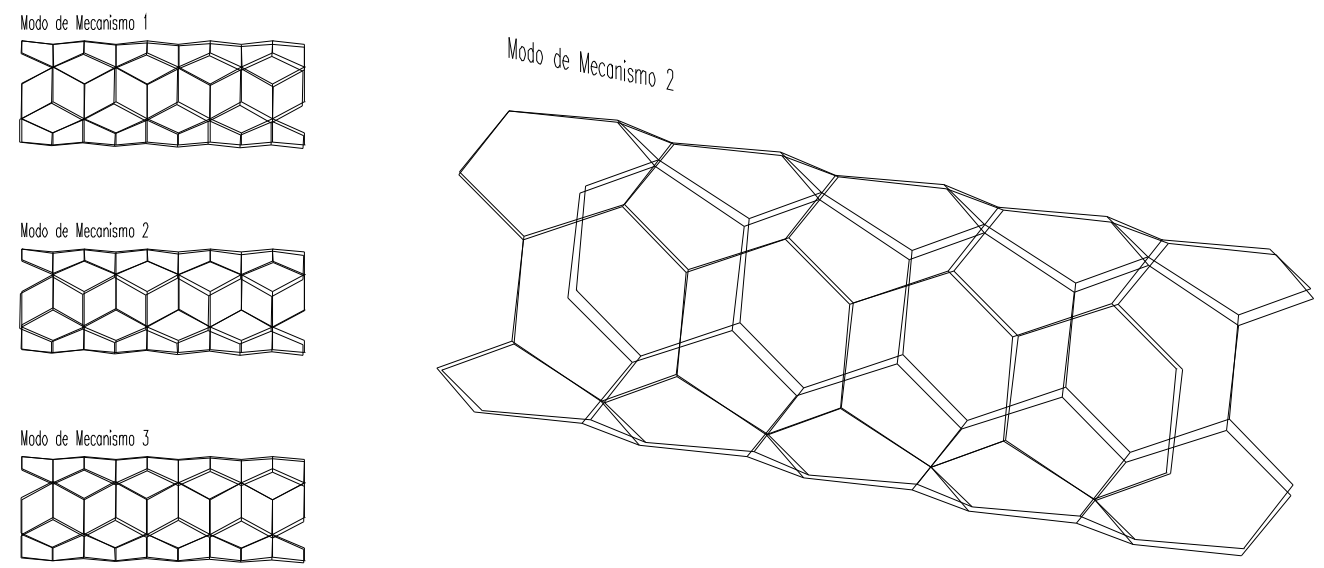

Figura 3.7: Modos de mecanismo $A C(3,3), n_{L}=4.5$ celdas

coacciones, el primer número indica el $n^{0}$ de apoyos introducidos y el segundo el $n^{o}$ de coacciones que introduce cada uno. Asimismo, se indica entre paréntesis la numeración de los nudos coaccionados o una descripción del esquema de coacciones asimilando cada CNT a una viga equivalente.

\begin{tabular}{lclcc}
\hline & $n_{L} / \mathrm{n}$-mod & Descripción coacciones & $\mathrm{N}^{0}$ mec. & $\mathrm{N}^{0}$ autotens. \\
\hline $\mathrm{AC}(3,3)$ & 4 & $6 \times 3$ (tipo ménsula) & 0 & 69 \\
$\mathrm{AC}(3,3)$ & 6 & $6 \times 3$ (tipo ménsula) & 0 & 105 \\
$\mathrm{AC}(4,4)$ & 6 & $6 \times 3$ (nudos 1 y 72) & 1 & 123 \\
$\mathrm{AC}(4,4)$ & 8 & $1 \times 3$ (nudo 1) & 3 & 170 \\
\hline $\mathrm{ZZ}(5,0)$ & 6 & $10 \times 3$ (tipo biempotrada) & 0 & 110 \\
$\mathrm{ZZ}(5,0)$ & 8 & $5 \times 3$ (tipo ménsula) & 0 & 125 \\
$\mathrm{ZZ}(6,0)$ & 6 & $1 \times 3$ (nudo 6) & 3 & 102 \\
$\mathrm{ZZ}(6,0)$ & 8 & $2 \times 3$ (nudos 1 y 5) & 1 & 139 \\
\hline $\mathrm{CH}(4,2)$ & 1 & $6 \times 3$ (tipo ménsula) & 0 & 72 \\
$\mathrm{CH}(4,2)$ & 1 & $1 \times 3$ (nudo 1) & 3 & 60 \\
$\mathrm{CH}(5,2)$ & 1 & $7 \times 3$ (tipo ménsula) & 0 & 64 \\
$\mathrm{CH}(5,2)$ & 1 & $2 \times 3$ (nudos 1 y 40) & 1 & 50 \\
\hline
\end{tabular}

Tabla 3.1: Resultados tipo descomposición SVD 


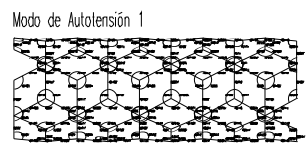

Modo de Autotensión 35

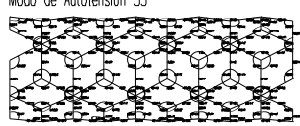

Modo de Autotensión 66
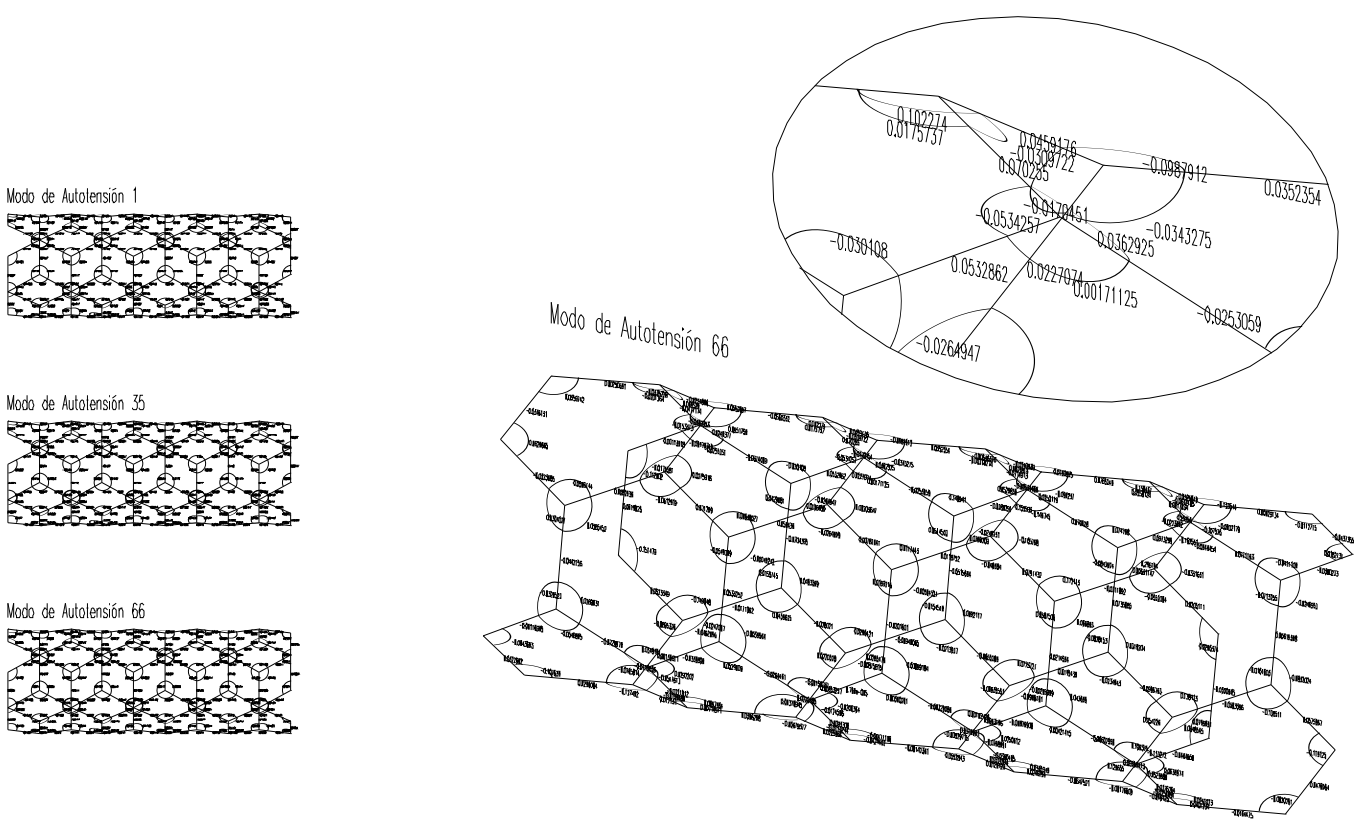

Figura 3.8: Ejemplos modos de autotensión $A C(3,3), n_{L}=4.5$ celdas

Como podemos deducir de los resultados obtenidos, los únicos modos de mecanismo presentes están asociados a movimientos globales de sólido rígido (mecanicidades externas). De hecho, en los casos donde aparece un modo de mecanismo, este corresponde a una rotación 3D tomando como eje la recta que une los dos nudos coaccionados. Igualmente, en los casos con tres modos de mecanismo, éstos están asociados con las tres rotaciones externas 3D de la estructura como conjunto.

Por otra parte, podemos aceptar que el modelo es altamente hiperestático, apareciendo 170 modos de autotensión independientes en el caso más extremo de todos los estudiados.

En definitiva, se puede asegurar que el modelo no presenta modos de mecanismo internos (críticos o finitos) y que será cinemáticamente admisible siempre que definamos unas coacciones externas capaces de bloquear cualquier movimiento conjunto de sólido rígido. Además, existirán infinitos sistemas de esfuerzos autoequilibrados que podrán ser obtenidos como combinación lineal del elevado número de modos básicos de autotensión. Por tanto, cualquier estado de tensiones o deformaciones iniciales que se pueda expresar a partir de la combinación lineal de estos modos básicos será aceptable desde el punto de vista estructural para introducir la preenergía en nuestro modelo. No obstante, queda fuera de los objetivos de este capítulo discriminar la idoneidad del estado de tensiones escogido. 



\section{Capítulo 4}

\section{Formulación general del modelo y su aplicación a nanotubos monocapa}

Se trata en este capítulo de desarrollar una formulación general en rigidez para el modelo stickspiral (ver [75]), de tal forma que las ecuaciones gobernantes obtenidas sean aplicables a cualquier sistema de elementos barra y muelle correctamente ensamblados. La ventaja fundamental de esta formulación reposa en su versatilidad, siendo capaz de reproducir la respuesta mecánica frente a cualquier sistema de cargas estáticamente admisible y cualquier quiralidad del nanotubo. Adicionalmente, la introducción de la preenergía a través de un sistema predeterminado de esfuerzos o deformaciones iniciales (pretensado) resulta inmediata.

Hipótesis adoptadas:

1. Los desplazamientos y las deformaciones se considerarán pequeños, pudiendo plantear el equilibrio en la geometría no deformada (análisis geométricamente lineal).

2. Los desplazamientos nodales serán exclusivamente traslaciones y las cargas nodales serán fuerzas. No se consideran grados de libertad de rotación ni la posibilidad de aplicar momentos como acciones externas.

3. Se descarta la existencia de modos de mecanismo internos (ver capítulo 3).

\subsection{Desarrollo energético de la formulación en rigidez}

\subsubsection{Ecuaciones cinemáticas}

Para una mayor claridad en la exposición, recuperamos en esta sección las ecuaciones cinemáticas fundamentales desarrolladas en el capítulo 3 .

\begin{tabular}{ll}
\hline Elemento barra & $\mathbf{C}_{l}^{a} \mathbf{u}^{a}=\epsilon^{a}$ \\
Elemento muelle & $\mathbf{C}_{l}^{1} \mathbf{u}^{1}=\epsilon^{1}$ \\
Sistema estructural & {$\left[\begin{array}{l}\mathbf{C}^{\lambda} \\
\mathbf{C}^{\Gamma}\end{array}\right]\{\mathbf{u}\}=\left\{\begin{array}{l}\epsilon^{\lambda} \\
\epsilon^{\Gamma}\end{array}\right\}$} \\
& {$\left[\begin{array}{ll}\mathbf{C}_{R} & \mathbf{C}_{A}\end{array}\right]\left\{\begin{array}{l}\mathbf{u}_{R} \\
\mathbf{u}_{A}\end{array}\right\}=\boldsymbol{\epsilon}$}
\end{tabular}

Tabla 4.1: Resumen de las ecuaciones cinemáticas 


\subsubsection{Ecuaciones de equilibrio}

Resumimos en la tabla 4.2 las ecuaciones de equilibrio obtenidas previamente mediante la ecuación de trabajos virtuales:

\begin{tabular}{ll}
\hline Elemento barra & $\mathbf{f}^{a}=n^{a} \mathbf{H}_{l}^{a}$ \\
Elemento muelle & $\mathbf{f}^{1}=m^{1} \mathbf{H}_{l}^{1}$ \\
Sistema estructural & $\overline{\mathbf{f}}=\left[\begin{array}{ll}\mathbf{H}^{\lambda} & \mathbf{H}^{\Gamma}\end{array}\right]\left\{\begin{array}{l}\mathbf{n} \\
\mathbf{m}\end{array}\right\}$ \\
& $\left\{\begin{array}{l}\overline{\mathbf{f}}_{R} \\
\overline{\mathbf{f}}_{A}\end{array}\right\}=\left[\begin{array}{l}\mathbf{H}_{R} \\
\mathbf{H}_{A}\end{array}\right] \mathbf{p}$
\end{tabular}

Tabla 4.2: Resumen de las ecuaciones de equilibrio

\subsubsection{Ecuaciones constitutivas}

\section{Elemento barra}

La ecuación constitutiva del elemento barra elemental se puede escribir de la forma:

$$
\epsilon^{a}=\epsilon_{0}^{a}+F^{a} n^{a}
$$

donde $F^{a}$ denota la flexibilidad de barra y $\epsilon_{0}^{a}$ representa el alargamiento inicial que puede experimentar la barra previamente a la actuación de las fuerzas externas.

Despejando el axil de barra obtenemos la expresión inversa, más habitual:

$$
n^{a}=\left(F^{a}\right)^{-1} \epsilon^{a}-\left(F^{a}\right)^{-1} \epsilon_{0}^{a}=K^{a} \epsilon^{a}-K^{a} \epsilon_{0}^{a}=K^{a} \epsilon^{a}+n_{0}^{a}
$$

donde:

$K^{a} \quad$ Rigidez longitudinal de barra

$n_{0}^{a} \quad$ Esfuerzo axil inicial de pretensado

\section{Elemento muelle}

La ecuación constitutiva del elemento resorte angular se puede expresar:

$$
\epsilon^{1}=\epsilon_{0}^{1}+F^{1} m^{1}
$$

donde se denomina $F^{1}$ a la flexibilidad de muelle y $\epsilon_{0}^{1}$ a la distorsión angular inicial previa a la actuación de las cargas exteriores.

Despejando el momento de muelle llegamos a la expresión:

$$
m^{1}=\left(F^{1}\right)^{-1} \epsilon^{1}-\left(F^{1}\right)^{-1} \epsilon_{0}^{1}=K^{1} \epsilon^{1}-K^{1} \epsilon_{0}^{1}=K^{1} \epsilon^{1}+m_{0}^{1}
$$

donde:

$K^{1} \quad$ Rigidez a la distorsión angular de muelle

$m_{0}^{1} \quad$ Momento inicial de pretensado 


\section{Sistema estructural completo}

Agrupando ordenadamente las ecuaciones (4.1) y (4.3) para cualquier conjunto de elementos adecuadamente ensamblados, podemos expresar matricialmente la ecuación constitutiva del sistema como:

$$
\epsilon=\epsilon_{0}+\mathbf{F p}
$$

donde:

F Matriz de flexibilidad del sistema

$\boldsymbol{\epsilon}_{0}$ Vector de predeformaciones (pretensado) previos a la actuación de las fuerzas externas.

Premultiplicando 4.5 por $\mathbf{F}^{-1}$ y despejando los esfuerzos internos, obtenemos la ecuación constitutiva inversa de la estructura completa:

$$
\mathbf{p}=\mathbf{F}^{-1} \boldsymbol{\epsilon}-\mathbf{F}^{-1} \boldsymbol{\epsilon}_{0}=\mathbf{K} \boldsymbol{\epsilon}-\mathbf{K} \epsilon_{0}=\mathbf{K} \epsilon+\mathbf{p}_{0}
$$

donde:

K Matriz constitutiva que contiene las rigideces individuales de cada elemento

$\mathbf{p}_{0} \quad$ Esfuerzos iniciales del pretensado o autotensión inicial.

Descomponiendo por bloques 4.6 podemos escribir:

$$
\left\{\begin{array}{l}
\mathbf{n} \\
\mathbf{m}
\end{array}\right\}=\left[\begin{array}{cc}
\mathbf{K}^{\lambda} & \mathbf{0} \\
\mathbf{0} & \mathbf{K}^{\Gamma}
\end{array}\right]\left\{\begin{array}{l}
\boldsymbol{\epsilon}^{\lambda} \\
\boldsymbol{\epsilon}^{\Gamma}
\end{array}\right\}+\left\{\begin{array}{l}
\mathbf{n}_{0} \\
\mathbf{m}_{0}
\end{array}\right\}
$$

donde:

$\mathbf{K}^{\lambda} \quad$ Matriz constitutiva que contiene las rigideces a axil de las barras

$\mathbf{K}^{\Gamma} \quad$ Matriz constitutiva que contiene las rigideces a momento de los muelles

$\mathbf{n}_{0} \quad$ Axiles del pretensado en las barras.

$\mathbf{m}_{0}$ Momentos del pretensado en los muelles.

En (4.7) se ha asumido implícitamente que la rigidez axil de las barras está desacoplada de la rigidez a momento en los muelles, como es habitual en el comportamiento geométricamente lineal. Además, las matrices $\mathbf{K}^{\lambda}$ y $\mathbf{K}^{\Gamma}$ son diagonales y contienen en la diagonal principal las rigideces de cada elemento. Aunque la introducción en la formulación de potenciales tipo Tersoff-Brenner (TB) [10], [95] es factible, una sencilla revisión de las ecuaciones (2.10) a 2.15) permite afirmar que los parámetros constitutivos derivados del potencial TB están acoplados, lo que se traduce en una formulación complicada de la matriz constitutiva $\mathbf{K}$. Por ello se ha simplificado la composición de esta matriz asumiendo parámetros desacoplados.

En el caso particular (por ejemplo, con el potencial AMBER) que las rigideces a axil $K^{\rho}=K^{\lambda}$ (cte) para $\rho=a, b, c, \ldots, n b$ y las rigideces a momento $K^{\rho}=K^{\Gamma}($ cte $)$ para $\rho=1,2,3, \ldots, n m$, las matrices constitutivas se pueden simplificar como:

$$
\mathbf{K}^{\lambda}=K^{\lambda} \mathbf{I}_{(n b \times n b)} \quad \mathbf{K}^{\Gamma}=K^{\Gamma} \mathbf{I}_{(n m \times n m)}
$$

sustituyendo (4.8) en (4.7) obtenemos:

$$
\left\{\begin{array}{l}
\mathbf{n} \\
\mathbf{m}
\end{array}\right\}=\left\{\begin{array}{l}
K^{\lambda} \boldsymbol{\epsilon}^{\lambda} \\
K^{\Gamma} \boldsymbol{\epsilon}^{\Gamma}
\end{array}\right\}+\left\{\begin{array}{l}
\mathbf{n}_{0} \\
\mathbf{m}_{0}
\end{array}\right\}
$$




\section{Parámetros constitutivos}

Dentro del marco del análisis geométricamente lineal de SWNTs que nos ocupa, hemos empleado dos potenciales diferentes en la formulación con el objetivo de averiguar la influencia que tiene la función potencial en la respuesta estructural final.

Dada una función potencial $U(\Delta r, \Delta \theta)$, se pueden definir las relaciones esfuerzo-deformación asociadas según:

$$
n=\frac{\partial U}{\partial(\Delta r)} \quad m=\frac{\partial U}{\partial(\Delta \theta)}
$$

y las rigideces tangentes como:

$$
K^{\lambda}=\frac{\partial^{2} U}{\partial(\Delta r)^{2}} \quad K^{\Gamma}=\frac{\partial^{2} U}{\partial(\Delta \theta)^{2}}
$$

Consideraremos que en las definiciones (4.10) y (4.11) las deformaciones están medidas desde la geometría inicial del nanotubo (no desde la lámina plana de grafeno), por tanto el pretensado no está incluido explícitamente en ellas. Aplicándolas a la expresión simplificada (2.5) del potencial $\boldsymbol{A M B E R}$ y teniendo en cuenta los parámetros (2.8), tenemos:

$$
\begin{array}{ll}
n=k_{r}(\Delta r)=K^{\lambda}(\Delta r) & m=k_{\theta}(\Delta \theta)=K^{\Gamma}(\Delta \theta) \\
K^{\lambda}=652 \frac{n N}{\text { enlace } \cdot n m} & K^{\Gamma}=0.876 \frac{n N \cdot n m}{\text { angulo.rad }}
\end{array}
$$

es decir, el potencial AMBER implica una relación lineal mecánica para cada elemento de la estructura, que permite la simplificación (4.9) de las ecuaciones constitutivas del sistema.

Por otra parte, si adoptamos el potencial Morse (2.16), las expresiones genéricas [74, ec. (3)] de los esfuerzos serán:

$$
\begin{aligned}
& n=2 \beta D_{e} e^{-\beta(\Delta r)}\left[1-e^{-\beta(\Delta r)}\right] \\
& m=k_{\theta}(\Delta \theta)\left[1+3 k_{s}(\Delta \theta)^{4}\right]
\end{aligned}
$$

Contrariamente a 4.12a), las relaciones esfuerzo-deformación (4.13) son no lineales, de forma que contradicen aparentemente las relaciones constitutivas (4.2) y (4.4) adoptadas en nuestro modelo. Sin embargo, esta no linealidad constitutiva será tratada mediante un algoritmo numérico que linealiza (4.13) de forma incremental empleando las siguientes rigideces tangentes:

$$
\begin{aligned}
& K^{\lambda}(\Delta r)=2 \beta^{2} D_{e} e^{-\beta(\Delta r)}\left[2 e^{-\beta(\Delta r)}-1\right] \\
& K^{\Gamma}(\Delta \theta)=k_{\theta}\left[1+15 k_{s}(\Delta \theta)^{4}\right]
\end{aligned}
$$

donde se han tomado los valores (2.19) para los parámetros implicados. Representamos en la figura 4.1 las expresiones (4.14), de donde se deduce que la influencia de la no linealidad constitutiva será mucho mayor en la interacción longitudinal que en la angular, coincidiendo con [74].

Para una mejor comparación cualitativa entre ambos potenciales, se representan esfuerzos frente a deformaciones asociadas en la figura 4.2. Como podemos observar, no habrá diferencias significativas entre potenciales mientras los alargamientos no superen los $0.07 \mathrm{~nm}\left(\varepsilon \simeq 5 \%\right.$ con $a_{0}=0.142$ $\mathrm{nm}$ ) y las distorsiones angulares los $0.3 \mathrm{rad}$. 


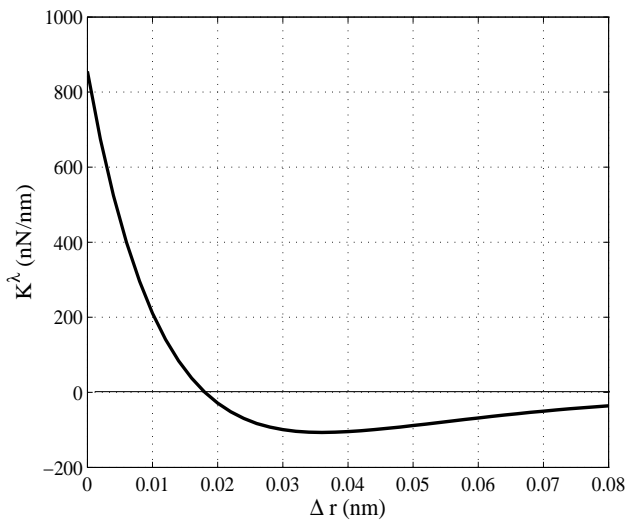

(a) Rigidez longitudinal enlace

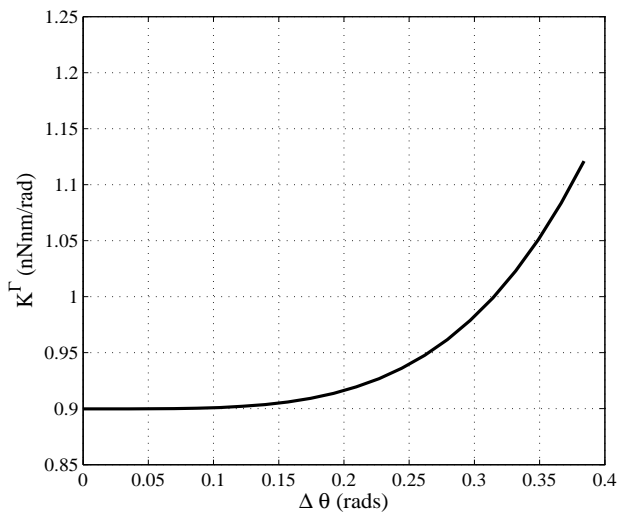

(b) Rigidez angular entre enlaces

Figura 4.1: Rigideces tangentes Morse

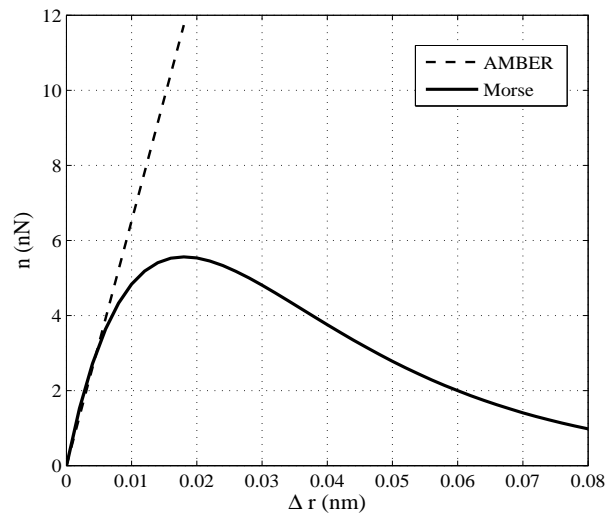

(a) Comparación axil genérico

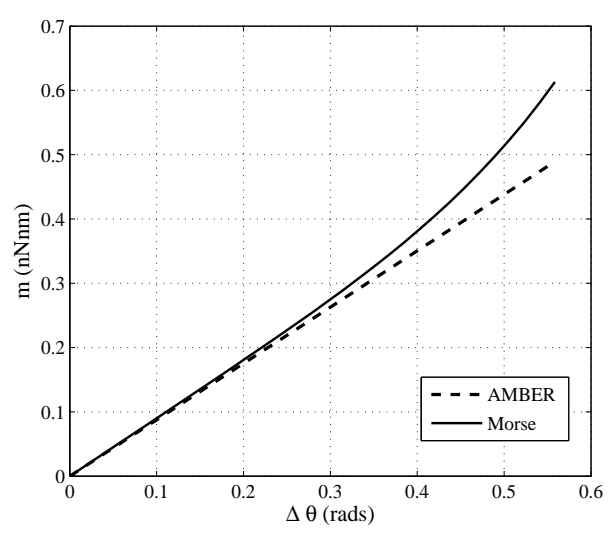

(b) Comparación momento genérico

Figura 4.2: Representación comparativa de las ecuaciones consitutivas

\subsubsection{Formulación matricial en rigidez}

\section{Elemento barra}

Sustituyendo la ecuación constitutiva (4.2) y las cinemáticas (3.5) en las de equilibrio (3.7) se obtiene la ecuación general de rigidez del elemento barra (ver figura 3.2):

$$
\mathbf{f}^{a}=K^{a} \mathbf{H}_{l}^{a} \mathbf{H}_{l}^{a T} \mathbf{u}^{a}+n_{0}^{a} \mathbf{H}_{l}^{a}=\widetilde{\mathbf{K}}_{l}^{a} \mathbf{u}^{a}+\mathbf{f}_{0}^{a}
$$

donde:

$\widetilde{\mathbf{K}}_{l}^{a} \quad$ Matriz de rigidez del elemento barra

$\mathbf{f}_{0}^{a} \quad$ Fracción de las fuerzas nodales iniciales debidas al pretensado $\mathrm{y}$ asociadas al alargamiento de barra 
Teniendo en cuenta la configuración de $\mathbf{H}_{l}^{a}$, podemos descomponer 4.15) en bloques de la forma:

$$
\left\{\begin{array}{c}
\mathbf{f}_{i}^{a} \\
\mathbf{f}_{j}^{a}
\end{array}\right\}=\left[\begin{array}{cc}
\widetilde{\mathbf{K}}_{i i}^{a} & \widetilde{\mathbf{K}}_{i j}^{a} \\
\widetilde{\mathbf{K}}_{j i}^{a} & \widetilde{\mathbf{K}}_{j j}^{a}
\end{array}\right]\left\{\begin{array}{c}
\mathbf{u}_{i} \\
\mathbf{u}_{j}
\end{array}\right\}+\left\{\begin{array}{c}
\mathbf{f}_{0 i}^{a} \\
\mathbf{f}_{0 j}^{a}
\end{array}\right\}=\left[\begin{array}{cc}
K^{a} \lambda^{a} \lambda^{a T} & -K^{a} \lambda^{a} \lambda^{a T} \\
-K^{a} \lambda^{a} \lambda^{a T} & K^{a} \lambda^{a} \lambda^{a T}
\end{array}\right]\left\{\begin{array}{c}
\mathbf{u}_{i} \\
\mathbf{u}_{j}
\end{array}\right\}+\left\{\begin{array}{c}
-n_{0}^{a} \lambda^{a} \\
n_{0}^{a} \lambda^{a}
\end{array}\right\}
$$

donde se entiende que los productos $\lambda^{a} \lambda^{a T}$ son productos tensoriales cuyo resultado es un tensor de segundo orden y dimensión 3.

\section{Elemento muelle}

Sustituyendo la ecuación constitutiva (4.2) y las cinemáticas (3.5) en las de equilibrio (3.7) se obtiene la ecuación general de rigidez del elemento muelle (ver figura 3.3 ):

$$
\mathbf{f}^{1}=K^{1} \mathbf{H}_{l}^{1} \mathbf{H}_{l}^{1^{T}} \mathbf{u}^{1}+m_{0}^{1} \mathbf{H}_{l}^{1}=\widetilde{\mathbf{K}}_{l}^{1} \mathbf{u}^{1}+\mathbf{f}_{0}^{1}
$$

donde:

$\widetilde{\mathbf{K}}_{l}^{1} \quad$ Matriz de rigidez del elemento muelle

$\mathbf{f}_{0}^{1} \quad$ Fracción de las fuerzas nodales iniciales debidas al pretensado y asociadas a la distorsión de muelle

Considerando la composición de la matriz $\mathbf{H}_{l}^{1}$, podemos descomponer (4.17) para escribir:

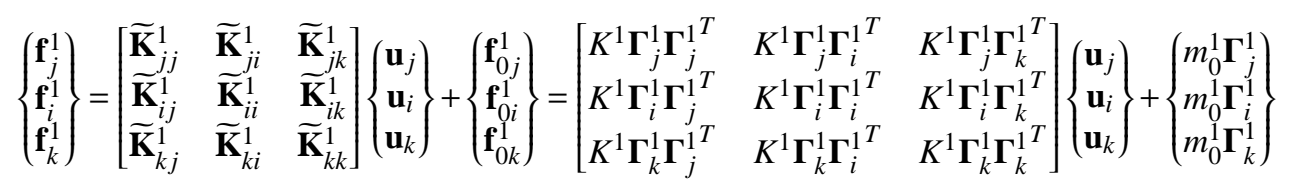

donde se entiende que los productos $\Gamma_{\alpha}^{1} \Gamma_{\beta}^{1{ }^{T}}$ son productos tensoriales cuyo resultado es un tensor de segundo orden y dimensión 3 .

\section{Unidad básica}

Considerando la unidad básica formada por dos elementos barra y un resorte angular entre ellos (figura 3.4), sustituimos las ecuaciones constitutivas (4.6) y las cinemáticas (3.27) en las de equilibrio (3.36), llegando a la expresión general en rigidez:

$$
\overline{\mathbf{f}}=\mathbf{H}_{l} \mathbf{K} \mathbf{H}_{l}^{T} \mathbf{u}+\mathbf{H}_{l} \mathbf{p}_{0}=\widetilde{\mathbf{K}}_{l} \mathbf{u}+\mathbf{f}_{0}
$$

donde:

$\widetilde{\mathbf{K}}_{l} \quad$ Matriz de rigidez de la unidad básica

$\mathbf{f}_{0} \quad$ Fuerzas nodales iniciales debidas al pretensado, calculadas a partir de las diferencias de longitud de enlace y las diferencias angulares entre enlaces desde la lámina de grafeno hasta el nanotubo, multiplicadas por las rigideces derivadas del potencial (sección 4.1.5). Veamos en detalle la configuración de la matriz $\widetilde{\mathbf{K}}_{l}$ :

$$
\begin{aligned}
\widetilde{\mathbf{K}}_{l} & =\mathbf{H}_{l} \mathbf{K} \mathbf{H}_{l}^{T}=\left[\begin{array}{ccc}
\lambda^{a} & \mathbf{0} & \boldsymbol{\Gamma}_{j}^{1} \\
-\lambda^{a} & -\lambda^{b} & \boldsymbol{\Gamma}_{i}^{1} \\
\mathbf{0} & \lambda^{b} & \boldsymbol{\Gamma}_{k}^{1}
\end{array}\right]\left[\begin{array}{ccc}
K^{a} & 0 & 0 \\
0 & K^{b} & 0 \\
0 & 0 & K^{1}
\end{array}\right]\left[\begin{array}{ccc}
\lambda^{a T} & -\lambda^{a T} & \mathbf{0} \\
\mathbf{0} & -\lambda^{b^{T}} & \lambda^{b^{T}} \\
\boldsymbol{\Gamma}_{j}^{1 T} & \boldsymbol{\Gamma}_{i}^{1^{T}} & \boldsymbol{\Gamma}_{k}^{1^{T}}
\end{array}\right]= \\
& =\left[\begin{array}{ccc}
K^{a} \lambda^{a} \lambda^{a T}+K^{1} \boldsymbol{\Gamma}_{j}^{1} \boldsymbol{\Gamma}_{j}^{1} & -K^{a} \boldsymbol{\lambda}^{a} \lambda^{a T}+K^{1} \boldsymbol{\Gamma}_{j}^{1} \boldsymbol{\Gamma}_{i}^{1^{T}} & K^{1} \boldsymbol{\Gamma}_{j}^{1} \boldsymbol{\Gamma}_{k}^{1^{T}} \\
-K^{a} \lambda^{a} \lambda^{a T}+K^{1} \boldsymbol{\Gamma}_{i}^{1} \boldsymbol{\Gamma}_{j}^{1} & K^{a} \lambda^{a} \lambda^{a T}+K^{b} \lambda^{b} \lambda^{b^{T}}+K^{1} \boldsymbol{\Gamma}_{i}^{1} \boldsymbol{\Gamma}_{i}^{1^{T}} & -K^{b} \lambda^{b} \lambda^{b^{T}}+K^{1} \boldsymbol{\Gamma}_{i}^{1} \boldsymbol{\Gamma}_{k}^{1^{T}} \\
K^{1} \boldsymbol{\Gamma}_{k}^{1} \boldsymbol{\Gamma}_{j}^{1^{T}} & -K^{b} \lambda^{b} \lambda^{b^{T}}+K^{1} \boldsymbol{\Gamma}_{k}^{1} \boldsymbol{\Gamma}_{i}^{1^{T}} & K^{b} \lambda^{b} \lambda^{b^{T}}+K^{1} \boldsymbol{\Gamma}_{k}^{1} \boldsymbol{\Gamma}_{k}^{1^{T}}
\end{array}\right]
\end{aligned}
$$


Comparando cada bloque con los contenidos en las ecuaciones (4.16) y 4.18, podemos reescribir $\widetilde{\mathbf{K}}_{l}$ como:

$$
\widetilde{\mathbf{K}}_{l}=\left[\begin{array}{ccc}
\widetilde{\mathbf{K}}_{j j}^{a}+\widetilde{\mathbf{K}}_{j j}^{1} & \widetilde{\mathbf{K}}_{j i}^{a}+\widetilde{\mathbf{K}}_{j i}^{1} & \widetilde{\mathbf{K}}_{j k}^{1} \\
\widetilde{\mathbf{K}}_{i j}^{a}+\widetilde{\mathbf{K}}_{i j}^{1} & \widetilde{\mathbf{K}}_{i i}^{a}+\widetilde{\mathbf{K}}_{i i}^{b}+\widetilde{\mathbf{K}}_{i i}^{1} & \widetilde{\mathbf{K}}_{i k}^{b}+\widetilde{\mathbf{K}}_{i k}^{1} \\
\widetilde{\mathbf{K}}_{k j}^{1} & \widetilde{\mathbf{K}}_{k i}^{b}+\widetilde{\mathbf{K}}_{k i}^{1} & \widetilde{\mathbf{K}}_{k k}^{b}+\widetilde{\mathbf{K}}_{k k}^{1}
\end{array}\right]
$$

de donde podemos concluir que se verifica el ensamblaje booleano habitual en el modelo de la tesis, considerando los muelles como elementos estructurales independientes. Además, puede observarse que la matriz de rigidez de la unidad básica es simétrica. Por supuesto, estas conclusiones pueden extenderse a cualquier sistema de elementos ensamblados de forma que no se permita ningún modo de mecanismo interno.

También conviene desarrollar el vector de fuerzas nodales iniciales $\mathbf{f}_{0}$ :

$$
\mathbf{f}_{0}=\mathbf{H}_{l} \mathbf{p}_{0}=\left[\begin{array}{ccc}
\lambda^{a} & \mathbf{0} & \boldsymbol{\Gamma}_{j}^{1} \\
-\lambda^{a} & -\lambda^{b} & \boldsymbol{\Gamma}_{i}^{1} \\
\mathbf{0} & \lambda^{b} & \boldsymbol{\Gamma}_{k}^{1}
\end{array}\right]\left\{\begin{array}{c}
n_{0}^{a} \\
n_{0}^{b} \\
m_{0}^{1}
\end{array}\right\}=\left\{\begin{array}{c}
\mathbf{f}_{0 j}^{a}+\mathbf{f}_{0 j}^{1} \\
\mathbf{f}_{0 i}^{a}+\mathbf{f}_{0 i}^{b}+\mathbf{f}_{0 i}^{1} \\
\mathbf{f}_{0 k}^{b}+\mathbf{f}_{0 k}^{1}
\end{array}\right\}
$$

Véase que las fuerzas nodales iniciales consisten en la adición de las fracciones de cada elemento estructural independiente. Es decir, el ensamblaje booleano también opera adecuadamente en el vector $\mathbf{f}_{0}$.

Comentario 4.1.1 La idea de adición obtenida en las fuerzas nodales iniciales también se verifica en las acciones nodales externas. Desarrollando (3.35) para la unidad básica, obtenemos:

$$
\left\{\begin{array}{c}
\overline{\mathbf{f}}_{j} \\
\overline{\mathbf{f}}_{i} \\
\overline{\mathbf{f}}_{k}
\end{array}\right\}=\left[\begin{array}{ccc}
\lambda^{a} & \mathbf{0} & \boldsymbol{\Gamma}_{j}^{1} \\
-\lambda^{a} & -\lambda^{b} & \boldsymbol{\Gamma}_{i}^{1} \\
\mathbf{0} & \lambda^{b} & \boldsymbol{\Gamma}_{k}^{1}
\end{array}\right]\left\{\begin{array}{c}
n^{a} \\
n^{b} \\
m^{1}
\end{array}\right\}=n^{a}\left\{\begin{array}{c}
\lambda^{a} \\
-\lambda^{a} \\
\mathbf{0}
\end{array}\right\}+n^{b}\left\{\begin{array}{c}
\mathbf{0} \\
-\lambda^{b} \\
\lambda^{b}
\end{array}\right\}+m^{1}\left\{\begin{array}{l}
\boldsymbol{\Gamma}_{j}^{1} \\
\boldsymbol{\Gamma}_{i}^{1} \\
\boldsymbol{\Gamma}_{k}^{1}
\end{array}\right\}=\left\{\begin{array}{c}
\mathbf{f}_{j}^{a} \\
\mathbf{f}_{i}^{a} \\
\mathbf{0}
\end{array}\right\}+\left\{\begin{array}{c}
\mathbf{0} \\
\mathbf{f}_{i}^{b} \\
\mathbf{f}_{k}^{b}
\end{array}\right\}+\left\{\begin{array}{c}
\mathbf{f}_{j}^{1} \\
\mathbf{f}_{i}^{1} \\
\mathbf{f}_{k}^{1}
\end{array}\right\}
$$

es decir, que las fuerzas externas a la unidad básica se componen a partir de la suma de las colaboraciones de cada elemento individual.

\section{Sistema estructural completo}

La obtención de la ecuación global de rigidez de cualquier sistema de elementos barra y muelle conectados adecuadamente es análoga a la de la unidad básica (4.19), extendiendo los conceptos de ensamblaje deducidos de las ecuaciones (4.21) y 4.22).

La introducción de las condiciones de contorno, con la consiguiente reducción del sistema global, se trata de forma idéntica a como se hizo en la sección 3.3.3 Recordamos en la figura 4.3 la descomposición en bloques adoptada para el campo de desplazamientos y el de acciones externas.

Sustituyendo las ecuaciones constitutivas (4.6) y las cinemáticas (3.37) en las de equilibrio (3.39), obtenemos una descomposición en bloques de la ecuación de rigidez global:

$$
\begin{gathered}
\left\{\begin{array}{l}
\overline{\mathbf{f}}_{R} \\
\overline{\mathbf{f}}_{A}
\end{array}\right\}=\left[\begin{array}{l}
\mathbf{H}_{R} \\
\mathbf{H}_{A}
\end{array}\right] \mathbf{K}\left[\begin{array}{ll}
\mathbf{H}_{R}^{T} & \mathbf{H}_{A}^{T}
\end{array}\right]\left\{\begin{array}{l}
\mathbf{u}_{R} \\
\mathbf{u}_{A}
\end{array}\right\}+\left[\begin{array}{l}
\mathbf{H}_{R} \\
\mathbf{H}_{A}
\end{array}\right] \mathbf{p}_{0}=\left[\begin{array}{ll}
\mathbf{H}_{R} \mathbf{K} \mathbf{H}_{R}^{T} & \mathbf{H}_{R} \mathbf{K} \mathbf{H}_{A}^{T} \\
\mathbf{H}_{A} \mathbf{K} \mathbf{H}_{R}^{T} & \mathbf{H}_{A} \mathbf{K} \mathbf{H}_{A}^{T}
\end{array}\right]\left\{\begin{array}{l}
\mathbf{u}_{R} \\
\mathbf{u}_{A}
\end{array}\right\}+\left\{\begin{array}{l}
\mathbf{H}_{R} \mathbf{p}_{0} \\
\mathbf{H}_{A} \mathbf{p}_{0}
\end{array}\right\} \\
\left\{\begin{array}{c}
\overline{\mathbf{f}}_{R} \\
\overline{\mathbf{f}}_{A}
\end{array}\right\}=\left[\begin{array}{cc}
\widetilde{\mathbf{K}}_{R} & \widetilde{\mathbf{K}}_{R A} \\
\widetilde{\mathbf{K}}_{A R} & \widetilde{\mathbf{K}}_{A}
\end{array}\right]\left\{\begin{array}{l}
\mathbf{u}_{R} \\
\mathbf{u}_{A}
\end{array}\right\}+\left\{\begin{array}{l}
\mathbf{f}_{0 R} \\
\mathbf{f}_{0 A}
\end{array}\right\}
\end{gathered}
$$

donde ()$_{A}$ denota magnitudes relativas a los nudos apoyados y ()$_{R}$ magnitudes relativas a los nudos no coaccionados. Teniendo en cuenta que $\mathbf{u}_{A}=\mathbf{0}$, podemos escribir el sistema reducido de rigidez como:

$$
\overline{\mathbf{f}}_{R}=\widetilde{\mathbf{K}}_{R} \mathbf{u}_{R}+\mathbf{f}_{0 R}
$$




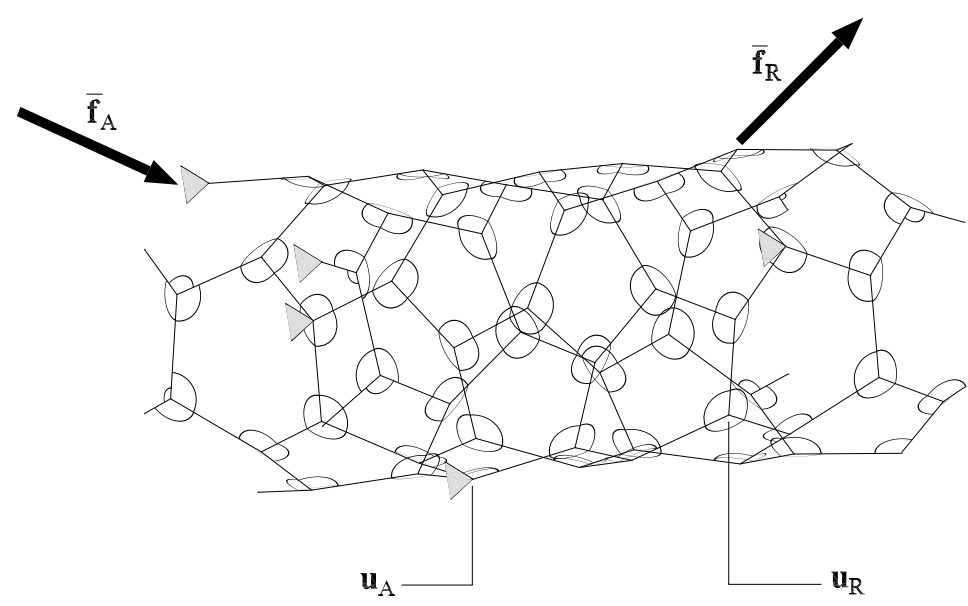

Figura 4.3: Esquema de cargas y apoyos en un $S W N T$

Finalmente, las reacciones se obtendrán mediante:

$$
\overline{\mathbf{f}}_{A}=\widetilde{\mathbf{K}}_{A R} \mathbf{u}_{R}+\mathbf{f}_{0 A}
$$

Comentario 4.1.2 Inicialmente, basamos el desarrollo de la formulación general en rigidez del presente modelo en una adaptación del método del equilibrio expuesto en [69] \$6,1] mediante matrices conexión. En dicho método, se ensambla previamente la matriz de equilibrio $\mathbf{H}_{l}$ a partir de la matriz conexión y de las matrices de equilibrio de los elementos individuales. Posteriormente se obtiene la matriz cinemática como $\mathbf{C}_{l}=\mathbf{H}_{l}^{T}$ y se procede según lo expuesto en las ecuaciones (4.24).

A pesar de tratarse de un método muy didáctico, ya que el ensamblaje de las matrices de equilibrio y cinemática es explícito, se abandonó porque requiere un alto número de operaciones con matrices dispersas. Por tanto, resulta poco eficaz desde el punto de vista de su rendimiento computacional.

\subsubsection{El problema de la predeformación de SWNTs}

Una lámina de grafeno plana consiste en un entramado hexagonal de átomos de $\mathrm{C}$ (ver figura 1.4), supuesta sin tensiones iniciales. Si consideramos idealmente un SWNT como el resultado de curvar una lámina de grafeno hasta que se cierre sobre sí misma formando un cilindro, es intuitivo que el SWNT estará en un estado de tensiones y deformaciones iniciales (pretensado o predeformación) producidas por la variación de las distancias interatómicas y de los ángulos entre enlaces en el proceso de curvado de la lámina, previamente a la actuación de cualquier acción externa. De hecho, el mismo concepto será aplicable a cada una de las capas que forman un MWNTs.

Se puede prever que dicho estado tensional será la causa fundamental (otra causa será la interacción de VDW) de la tendencia a mantener la geometría cilíndrica del CNT en ausencia de cargas externas. En consecuencia, deberá añadirse al estado de tensiones generado por cualquier acción externa hasta conseguir el estado definitivo de tensiones (y de deformaciones asociadas).

En la mayor parte de referencias consultadas, este pretensado se introduce en el cálculo mediante tratamientos energéticos. Por ejemplo, Iijima et. al. [43] (y casi simultáneamente Yakobson et. al. [115]) introducen el concepto de "exceso de energía de deformación" como la diferencia entre la energía de cada átomo en el CNT y su correspondiente energía de cohesión en una lámina plana de grafeno supuesta esta infinita. En esta dirección, Hernández et. al. [37], Jiang et. al. [44], Pantano et. al. [81] y Robertson et. al. [89] entre otros, establecen que la energía de deformación respecto a 
una lámina de grafeno plana, es proporcional a $1 / R^{2}$ (donde $R$ es el radio del CNT) y se mantiene constante frente a otros aspectos del entramado, como la quiralidad. Se puede deducir entonces que la influencia de la predeformación será mucho más importante en CNTs de pequeño radio.

De hecho, Tersoff y Ruoff [96] estudian la influencia del radio en la deformación transversal debida a la interacción de Van der Waals en un conjunto ordenado de SWNTs y concluyen que el aplanamiento de la sección transversal en geometrías sensiblemente hexagonales es tanto mayor conforme aumenta el radio del CNT. En cambio, en SWNTs de pequeño radio $(R<10 \AA ̊)$ donde el efecto del pretensado tiene un peso relativo mayor que la interacción VDW, los SWNTs conservan la geometría cilíndrica. En esta línea, Hertel et. al. [38] validan experimentalmente mediante AFM sus previsiones teóricas mediante MM y teoría del continuo respecto a la deformabilidad transversal de SWNTs tendidos en un sustrato. Aunque no mencionan explícitamente el concepto de predeformación, concluyen que la deformación transversal será mayor con el radio, como cabía esperar según [96].

Por otra parte, Harik et. al. [34] aclaran que la utilización del modelo viga en el tratamiento de los resultados experimentales que dependen del estado de tensiones del CNT, debe ser afectado por la curvatura del CNT (incluyendo la modificación que esta produce en el estado tensional), sobre todo en CNTs de radio reducido. Asimismo, el modelo lámina es validado mediante cálculos MD por Wang et. al. [103, 104] empleando el concepto de preenergía introducido por Yakobson [115].

Dada la complejidad del entramado hexagonal de átomos en el SWNT, cabe esperar que la configuración inicial de equilibrio asociada al mínimo de la energía total del sistema en ausencia de cargas externas, difiera sensiblemente del cilindro ideal. En las simulaciones atómicas (MD, tight-binding o $a b$ initio) esta situación se suele tener en cuenta (por ejemplo en [6], [43], [64], [94], [115]) reproduciendo el proceso de carga escalonadamente y minimizando la energía total en cada etapa mediante algún algoritmo numérico (por ejemplo, relajación dinámica).

Algo similar ocurriría en los MWNTs en caso de que la separación entre sus capas constituyentes no fuera la distancia interplanar de equilibrio $(t=0.34 \mathrm{~nm})$ del grafito [121]. En tal caso, un proceso de relajación dinámica determinaría la posición de los átomos previa a la actuación de cargas externas, y las tensiones iniciales se deberían a una situación de compromiso entre la interacción entre capas y la curvatura propia de cada capa.

En el presente modelo MSM, supondremos (tal como proponen Dresselhauss [26], Ghang y Gao [14], Xiao et. al. [114], Meo y Rossi [74, ec (8)] y Jiang et. al. [44]) que los núcleos atómicos están localizados en un sistema de coordenadas convectivas sobre la lámina de grafeno, acompañando a dichas coordenadas en el proceso ideal de curvado hasta la geometría cilíndrica. Por tanto, los átomos de C están dispuestos sobre una superficie cilíndrica ideal en el CNT, mientras las barras que representan los enlaces covalentes irán orientadas según las secantes al cilindro que unen dos átomos de $\mathrm{C}$ del entramado hexagonal. Esta definición extensamente utilizada de la geometría inicial permite adoptar un único estado de tensiones iniciales de entre las infinitas posibilidades estáticamente admisibles.

La introducción del pretensado en nuestra formulación se ha realizado como sigue: Calculamos las componentes de alargamiento de cada barra como la diferencia entre la longitud de enlace en el CNT y en la lámina de grafend]. Análogamente obtendremos las distorsiones angulares iniciales como la diferencia de ángulos entre dos enlaces contiguos en el CNT y en la lámina de grafeno plana (donde teóricamente forman $\pi / 6$ ). Todas estas componentes de deformación correctamente ordenadas se introducen en el vector de predeformaciones $\epsilon_{0}$ de la ecuación (4.5), que premultiplicado por la matriz constitutiva $\mathbf{K}$ proporcionará el vector de esfuerzos iniciales $\mathbf{p}_{0}$ de (4.6). Siguiendo a partir de ese punto la formulación desarrollada, el pretensado queda incluido en el cálculo.

${ }^{1}$ adoptada habitualmente como $a_{0}=1.42 \AA$, véase [10] y [26] 
Comentario 4.1.3 La ecuación constitutiva (4.5) trata el problema a mediante un sistema de deformaciones impuestas en la geometría inicial $\boldsymbol{\epsilon}_{0}$, que deberán ser medidas tomando como origen de deformaciones la geometría cilíndrica del nanotubo. En particular, se asume que los elementos barra han experimentado alargamientos impuestos (tomando como origen la longitud de la secante) que se traducirán en un sistema de compresiones iniciales. Análogamente, las distorsiones impuestas en los elementos muelle vienen medidas tomando como origen el ángulo que forman dos enlaces contiguos en el SWNT.

En conclusión, el conjunto de esfuerzos iniciales definido a partir de la ecuación (4.6) como $\mathbf{p}_{0}=-\mathbf{K} \boldsymbol{\epsilon}_{0}$ tenderá a "estabilizar"la sección transversal, es decir, a aumentar el diámetro del nanotubo y a oponerse a posibles ovalizaciones transversales del cilindro.

Comentario 4.1.4 Cabe señalar que en los SWNTs Chiral modelizados habrá algunas barras contiguas a los bordes que no representarán realmente la unión de dos átomos de $C$, ya que se han definido secciones transversales planas en los extremos. Sin embargo, en estos elementos barra más cortos se ha introducido igualmente el pretensado ya que representan parte de un enlace real. Teniendo en cuenta que ambos extremos de cada una de estas barras están en la superficie cilíndrica, pero uno de ellos no representa un átomo de C, introducir el pretensado en la forma descrita producirá una cierta imprecisión local de borde en los extremos del SWNT.

\subsection{Implementación numérica}

La implementación numérica de la formulación general del modelo (análisis lineal geométrico) se ha realizado en dos fases. Por una parte, la generación de la geometría inicial y la salida gráfica de resultados ha sido programada en VisualLISP, mientras el núcleo de cálculo que contiene las ecuaciones fundamentales se ha desarrollado en lenguaje $\mathrm{C}++$.

Como se ha mencionado previamente, se tratan dos cuestiones fundamentales: la importancia de la elección de la función potencial y la influencia de la introducción de esfuerzos o deformaciones iniciales (pretensado) en la respuesta estructural de SWNTs. De esta forma, se han programado cuatro situaciones diferentes.

\subsubsection{Potencial AMBER}

La forma más sencilla de introducir la interacción atómica en el código consiste en considerar rigideces constantes (con los valores (4.12b) ) para cada elemento estructural. Para ello se ha adoptado el potencial armónico AMBER, dando lugar a un comportamiento lineal mecánico o constitutivo. En la figura 4.4 describimos gráficamente el sencillo (no iterativo) diagrama de flujo empleado en este caso. Los superíndices $\alpha, \beta$ denotan los elementos barra y muelle respectivamente.

Se han desarrollado dos programas con potencial AMBER (uno con, y otro sin esfuerzos iniciales) para investigar la influencia del pretensado en la respuesta de SWNTs. Estas fuerzas iniciales se han introducido a través de los segundos términos en las ecuaciones (4.7) y (4.25). No obstante, el diagrama de la figura 4.4 es válido para ambos cálculos. 


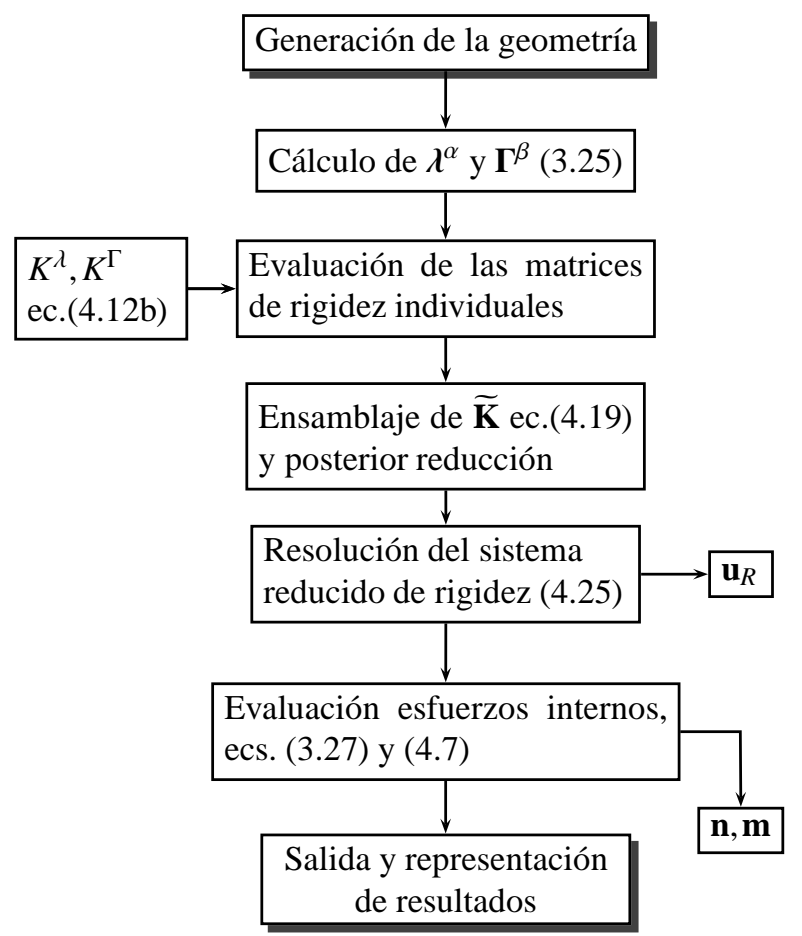

Figura 4.4: Diagrama de flujo no iterativo, potencial AMBER

\subsubsection{Potencial Morse}

La introducción del potencial de Morse en las ecuaciones fundamentales es completamente diferente del caso anterior, ya que las relaciones esfuerzo-deformación (4.13) son no lineales y llevan a un comportamiento no lineal constitutivo del modelo. Esta no linealidad se trata mediante un procedimiento iterativo que se esquematiza en la figura 4.5. basado en los métodos denominados tipo Newton [29] destinados a resolver numéricamente problemas no lineales. Los superíndices $k$ denotan el contador de la iteración y los índices $\alpha, \beta$ representan los elementos barra y muelle respectivamente.

En el marco del algoritmo numérico diseñado, las ecuaciones constitutivas (4.13) se linealizan en forma incremental según:

$$
\Delta n_{\alpha}^{(k)}=K_{\alpha}^{\lambda^{(k)}} \Delta r_{\alpha}^{(k)} \quad \Delta m_{\beta}^{(k)}=K_{\beta}^{\Gamma^{(k)}} \Delta \theta_{\beta}^{(k)}
$$

donde $K_{\alpha}^{\lambda^{(k)}}, K_{\beta}^{\Gamma^{(k)}}$ se calculan mediante las expresiones (4.14) al principio de la iteración $k$ y $\Delta r_{\alpha}^{(k)}, \Delta \theta_{\beta}^{(k)}$ serán los incrementos de deformación durante la misma iteración. La linealización (4.27) es inmediata sin más que combinar las definiciones (4.10) y 4.11), y aproximar los diferenciales resultantes por incrementos.

Llamando $u_{\rho}$ a una componente genérica de $\mathbf{u}_{R}$ al inicio de cada iteración, definimos el error absoluto en la componente $\rho$ de $\mathbf{u}_{R}$ como:

$$
E_{\rho}=\left|\Delta u_{\rho}\right| \quad \rho=1,2, \ldots, n
$$

donde $n$ es el número total de g.d.l. incluidos en el sistema lineal de rigidez reducido. De esta forma, el criterio de convergencia adoptado es:

$$
\left\|\Delta \mathbf{u}_{R}\right\|=\operatorname{máx}\left\{E_{\rho}\right\}<\varepsilon=10^{-6}
$$




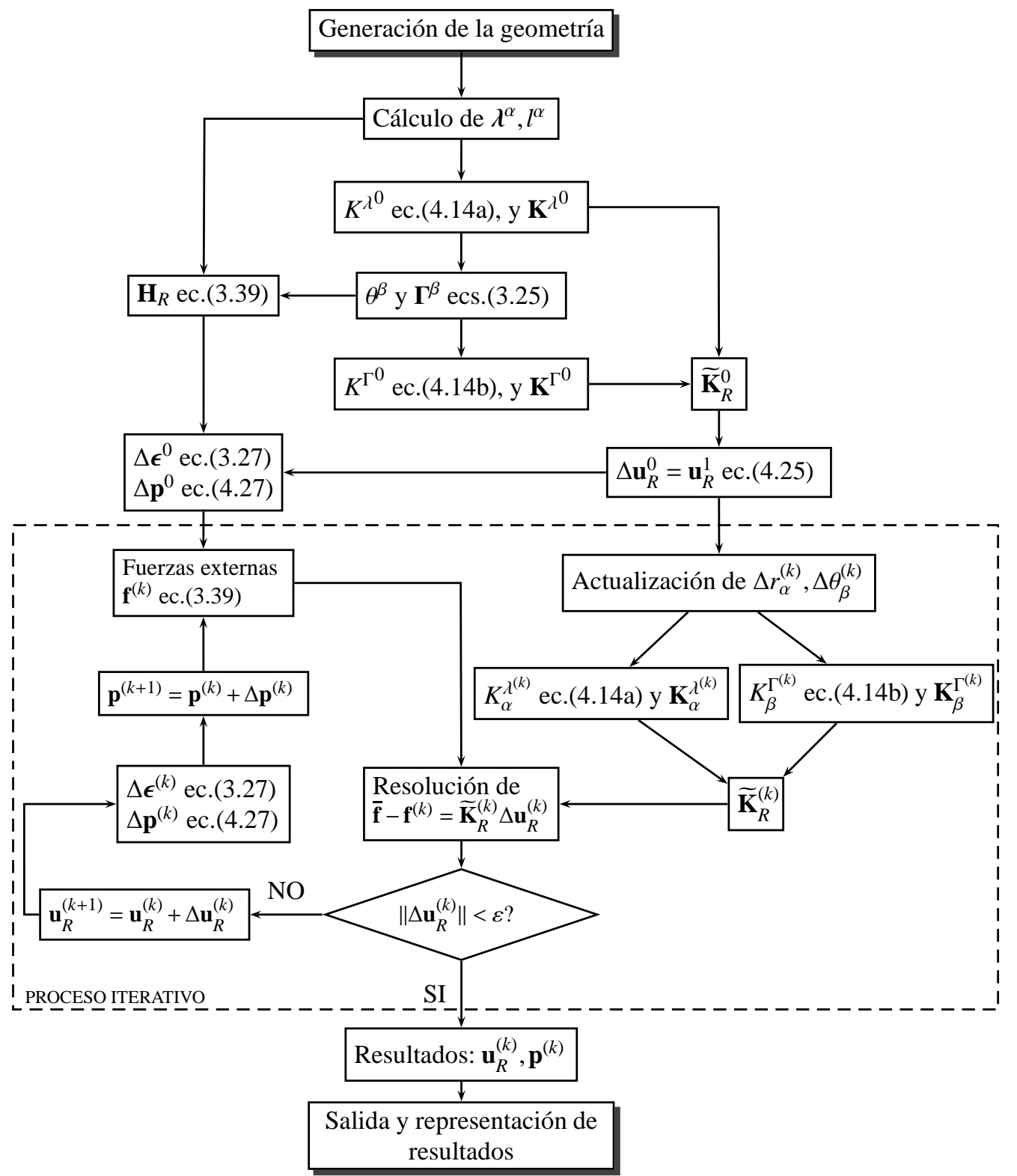

Figura 4.5: Procedimiento iterativo para introducir el potencial Morse

Nótese que $K^{\lambda^{0}}, K^{\Gamma^{0}}$ son los parámetros constitutivos de rigidez en la geometría no deformada del nanotubo. Si el pretensado no se considera en el cálculo, su evaluación se efectuará sustituyendo $\Delta r=\Delta \theta=0$ en las ecuaciones (4.14), pero si se incluyen las tensiones iniciales se empleará $\Delta r=\Delta r_{0}$ y $\Delta \theta=\Delta \theta_{0}$ (deformaciones iniciales desde la lámina de grafeno) en su lugar.

Además, se debe subrayar que $\mathbf{K}_{\alpha}^{\lambda^{(k)}}, \mathbf{K}_{\beta}^{\Gamma^{(k)}}$ son actualizadas exclusivamente a través de $K_{\alpha}^{\lambda^{(k)}}, K_{\beta}^{\Gamma^{(k)}}$ (actualización constitutiva), ya que los vectores $\lambda^{\alpha}, \Gamma^{\beta}$ implicados son tomados de la geometría inicial. Se trata entonces de un análisis lineal en cuanto a la geometría, coherente con la hipótesis de pequeños desplazamientos adoptada inicialmente. 


\subsection{Resultados y discusión}

En esta sección se aplica la nueva formulación general en rigidez del modelo stick-spiral al estudio del comportamiento estructural de SWNTs a tracción, compresión flexión y torsión. Las dimensiones de los nanotubos ensayados en cada caso de carga se resumen en la tabla 4.3. donde se han escogido diámetros y esbelteces similares con el propósito de comparar los resultados obtenidos.

\begin{tabular}{lccc}
\hline Chirality & $\mathrm{d}(\mathrm{nm})$ & $\mathrm{L}(\mathrm{nm})$ & $\mathrm{L} / \mathrm{d}$ \\
\hline $\mathrm{ZZ}(9,0)$ & 0.7046 & 8.5910 & 12.2 \\
$\mathrm{ZZ}(11,0)$ & 0.8612 & 10.2950 & 12.0 \\
$\mathrm{ZZ}(13,0)$ & 1.0178 & 12.2120 & 12.0 \\
\hline $\mathrm{AC}(5,5)$ & 0.6780 & 8.1164 & 12.0 \\
$\mathrm{AC}(7,7)$ & 0.9492 & 11.3138 & 11.9 \\
$\mathrm{AC}(8,8)$ & 1.0848 & 12.7895 & 11.8 \\
\hline $\mathrm{CH}(7,4)$ & 0.7541 & 8.9009 & 11.8 \\
$\mathrm{CH}(8,5)$ & 0.8879 & 9.6767 & 10.9 \\
$\mathrm{CH}(10,6)$ & 1.0960 & 11.9272 & 10.9 \\
\hline
\end{tabular}

Tabla 4.3: Dimensiones y quiralidades de los SWNTs ensayados

Se realiza a continuación un estudio de algunos parámetros mecánicos equivalentes mediante el modelo de la tesis. Aún admitiendo que la obtención de estos parámetros mediante un modelos atómico discreto es muy controvertida, debido a su dependencia del espesor de pared equivalente (tomando aquí el valor habitual de $t=0.34 \mathrm{~nm}$ [114], [120]), se calculan sus valores a efectos de comparación con los resultados presentes en la literatura.

En todos los casos se adoptan condiciones de sustentación tipo ménsula, de acuerdo con [74] y [120, fig 4], debido a su sencillez de implementación y posterior interpretación de los resultados numéricos. Tales coacciones se introducido mediante apoyos articulados en los nudos del extremo izquierdo del SWNT, coherentes con los grados de libertad de traslación considerados en nuestro modelo.

Por otra parte, el extremo libre ha sido cargado con un sistema de cargas puntuales nodales estáticamente equivalentes al esfuerzo global aplicado en cada caso. Por supuesto, no existe un único sistema de cargas puntuales para cada esfuerzo y se requiere alguna hipótesis adicional, particular para cada caso de carga. Se proporciona más adelante la descripción detallada de cada una de estas hipótesis y valores numéricos para las cargas puntuales obtenidas.

\subsubsection{Comportamiento a tracción}

El comportamiento a tracción se analiza mediante el trazado de curvas tensión equivalentedeformación para los SWNTs de la tabla 4.3. Los escalones de tensión a tracción se toman 20, 40, 60, 80, $100 \mathrm{GPa}$.

La deformación longitudinal equivalente del CNT como pieza se calcula:

$$
\epsilon_{x}=\frac{\Delta L}{L}
$$

donde:

$\Delta L$ variación de longitud del nanotubo, calculado como el desplazamiento longitudinal medio en el extremo libre.

$L \quad$ Longitud inicial del nanotubo

La tensión equivalente se evalúa según:

$$
\sigma_{x}=\frac{F_{t}}{\pi d t}
$$


donde:

$F_{t} \quad$ Fuerza total aplicada en la dirección longitudinal

$d \quad$ Diámetro del nanotubo

$t \quad$ Espesor de pared, estimado en $0.34 \mathrm{~nm}$

Las cargas puntuales que actúan en los nudos del extremo libre se obtienen a partir de $R_{i}=F_{t} / N_{t}$, donde $N_{t}$ es el número de átomos en la dirección perimetral de la circunferencia del cilindro en dicho extremo. De hecho, todas las cargas puntuales a tracción tendrán el mismo valor dentro de cada simulación. Estos valores se proporcionan en la tabla 4.4

\begin{tabular}{ccccccc} 
& $R_{i}(\mathrm{nN}) F_{t}(\mathrm{nN})$ & $R_{i}(\mathrm{nN})$ & $F_{t}(\mathrm{nN})$ & \multicolumn{2}{c}{$R_{i}(\mathrm{nN})$} & $F_{t}(\mathrm{nN})$ \\
\hline$\sigma_{x}(\mathrm{Gpa})$ & \multicolumn{2}{c}{$\mathrm{ZZ}(9,0)$} & \multicolumn{2}{c}{$\mathrm{ZZ}(11,0)$} & \multicolumn{2}{c}{$\mathrm{ZZ}(13,0)$} \\
\hline 20 & 1.67 & 15.05 & 1.67 & 18.40 & 1.67 & 21.74 \\
40 & 3.34 & 30.10 & 3.34 & 36.79 & 3.34 & 43.48 \\
60 & 5.02 & 45.16 & 5.02 & 55.19 & 5.02 & 65.23 \\
80 & 6.69 & 60.21 & 6.69 & 73.59 & 6.69 & 86.97 \\
100 & 8.36 & 75.26 & 8.36 & 91.99 & 8.36 & 108.71 \\
\hline$\sigma_{x}(\mathrm{Gpa})$ & \multicolumn{2}{c}{$\mathrm{AC}(5,5)$} & \multicolumn{2}{c}{$\mathrm{AC}(7,7)$} & \multicolumn{2}{c}{$\mathrm{AC}(8,8)$} \\
\hline 20 & 1.45 & 14.48 & 1.45 & 20.28 & 1.45 & 23.17 \\
40 & 2.90 & 28.97 & 2.90 & 40.56 & 2.90 & 46.35 \\
60 & 4.35 & 43.45 & 4.35 & 60.83 & 4.35 & 69.52 \\
80 & 5.79 & 57.94 & 5.79 & 81.11 & 5.79 & 92.70 \\
100 & 7.24 & 72.42 & 7.24 & 101.39 & 7.24 & 115.87 \\
\hline$\sigma_{x}(\mathrm{Gpa})$ & $\mathrm{CH}(7,4)$ & \multicolumn{2}{c}{$\mathrm{CH}(8,5)$} & $\mathrm{CH}(10,6)$ \\
\hline 20 & 1.46 & 16.11 & 1.46 & 18.97 & 1.46 & 23.41 \\
40 & 2.93 & 32.22 & 2.92 & 37.93 & 2.93 & 46.83 \\
60 & 4.39 & 48.33 & 4.38 & 56.90 & 4.39 & 70.24 \\
80 & 5.86 & 64.44 & 5.84 & 75.87 & 5.85 & 93.66 \\
100 & 7.32 & 80.55 & 7.30 & 94.84 & 7.32 & 117.07 \\
\hline
\end{tabular}

Tabla 4.4: Cargas puntuales aplicadas en simulaciones a tracción

Se representan en las figuras 4.6 y 4.7 las curvas tensión-deformación a tracción obtenidas 2. En cada gráfica individual se han representado comparativamente los resultados de las cuatro situaciones desarrolladas, tanto respecto al potencial considerado como a las tensiones iniciales. Destinado a la validación del modelo de la tesis, se reproducen también los resultados publicados en algunas referencias mediante formulaciones parciales del modelo MSM stick-spiral. En particular, para los SWNTs ZigZag (ZZ) y Armchair (AC) se introducen los resultados de Natsuki et. al. [78] procesados con el potencial AMBER, junto con los de Natsuki y Endo [77] procesados con el potencial Morse. En ninguno de los dos trabajos se incluye el pretensado. Por otra parte, para los SWNTs Chiral $(\mathrm{CH})$ se reprodujeron los resultados de Ghang et. al. [15], aunque introduciendo las constantes del AMBER en la formulación, para una comparación más consistente.

Como cabía esperar, el comportamiento asociado al potencial AMBER es lineal en todos los casos (rigideces de enlace constantes), sin embargo el potencial Morse también produce un comportamiento lineal, debido a que las rigideces tangentes apenas varían en el intervalo de cargas considerado. En general, queda patente que nuestros resultados coinciden sensiblemente con los obtenidos en las referencias consultadas. A pesar de ello, se pueden detectar ciertas diferencias con [77] a partir de $\varepsilon_{x}=6 \%$ en nanotubos ZZ y de $\varepsilon_{x}=8 \%$ en nanotubos $\mathrm{AC}$, ya que dicho trabajo plantea el equilibrio del elemento representativo (que incluye unos pocos átomos y sus respectivos enlaces) en la geometría deformada, efectuando un análisis no lineal geométrico local.

\footnotetext{
${ }^{2}$ En adelante, indicaremos con (p) los resultados que incluyen el pretensado
} 


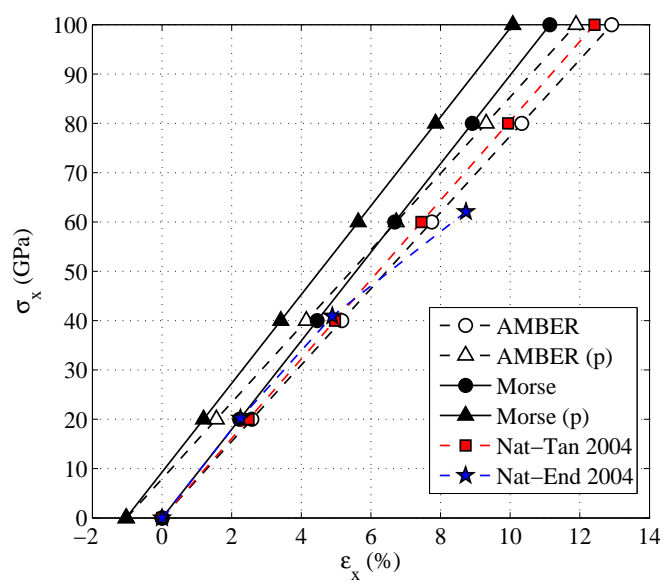

(a) $\mathrm{ZZ}(9,0)$ tracción

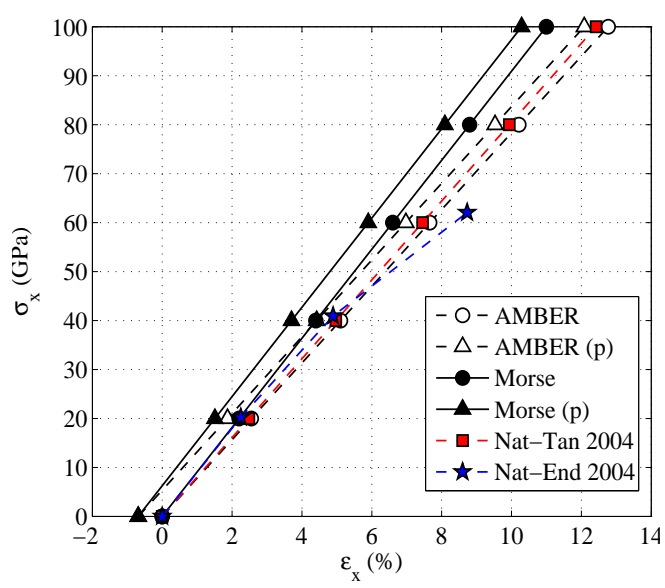

(c) ZZ(11,0) tracción

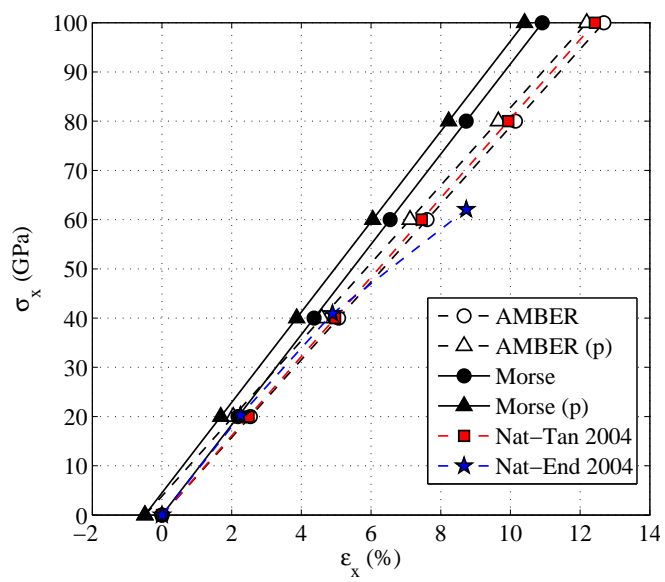

(e) ZZ $(13,0)$ tracción

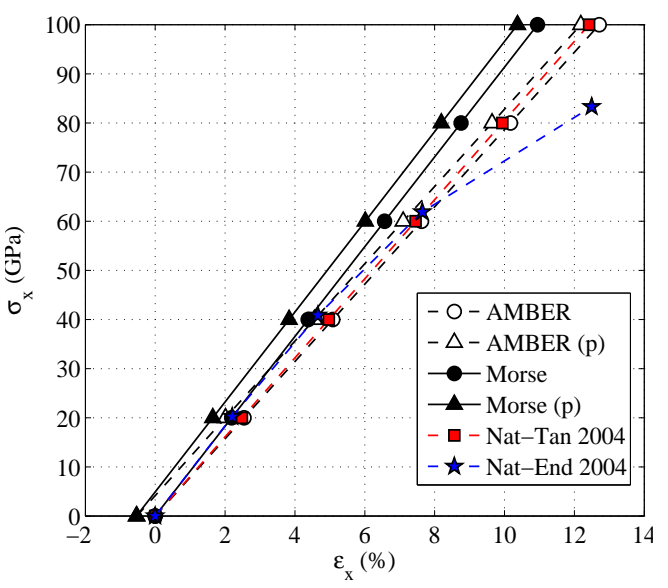

(b) AC(5,5) tracción

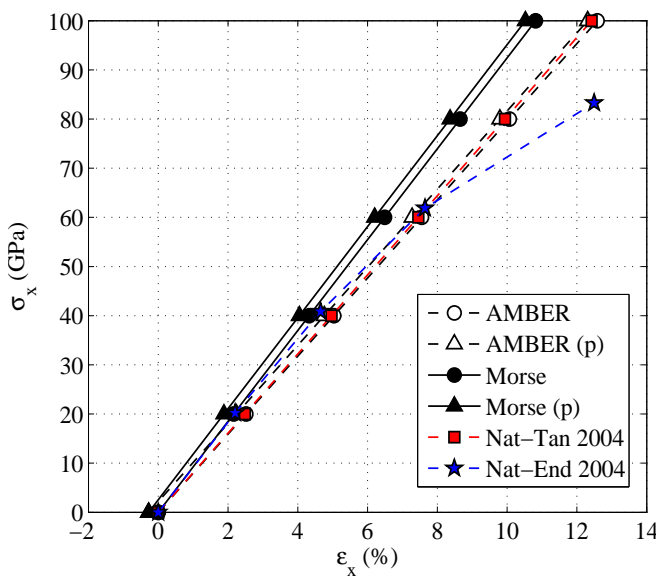

(d) $\operatorname{AC}(7,7)$ tracción

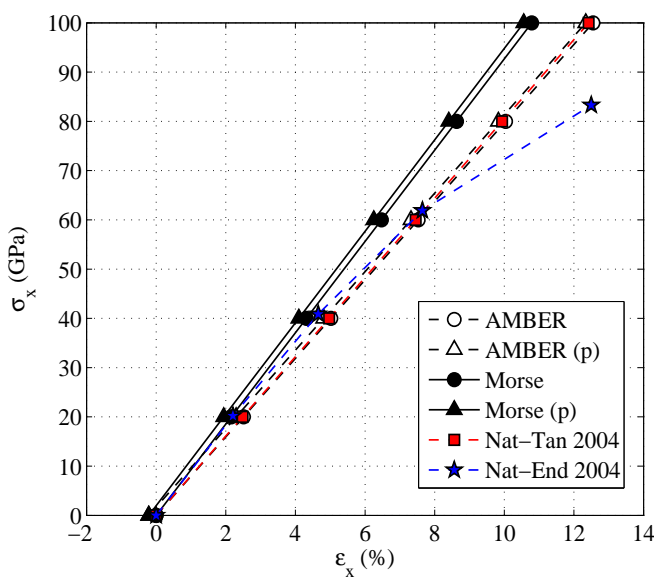

(f) $\mathrm{AC}(8,8)$ tracción

Figura 4.6: SWNTs Armchair y ZigZag a tracción, comparación con [77] y 78] 


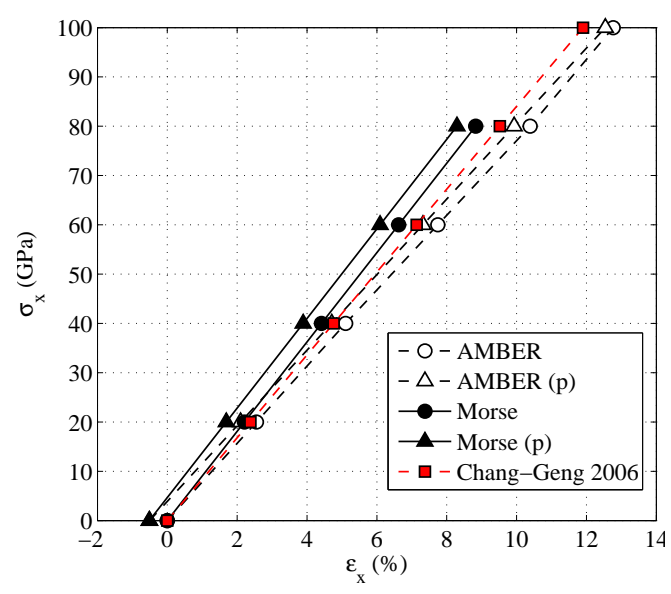

(a) $\mathrm{CH}(7,4)$ tracción

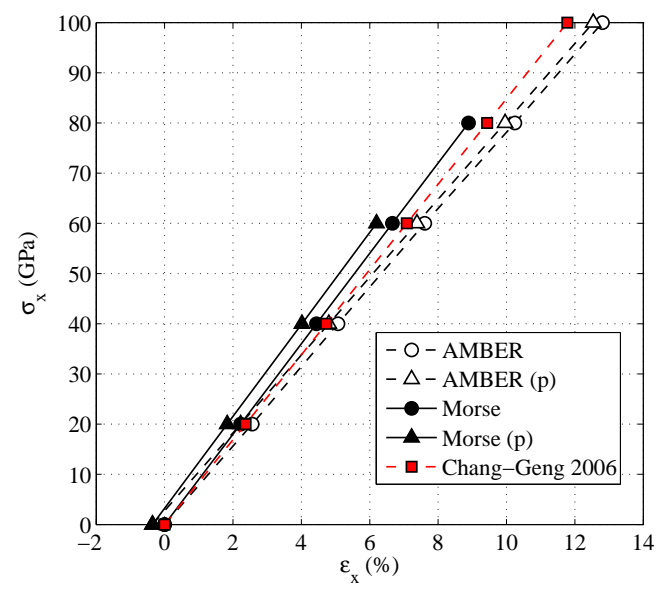

(b) $\mathrm{CH}(8,5)$ tracción

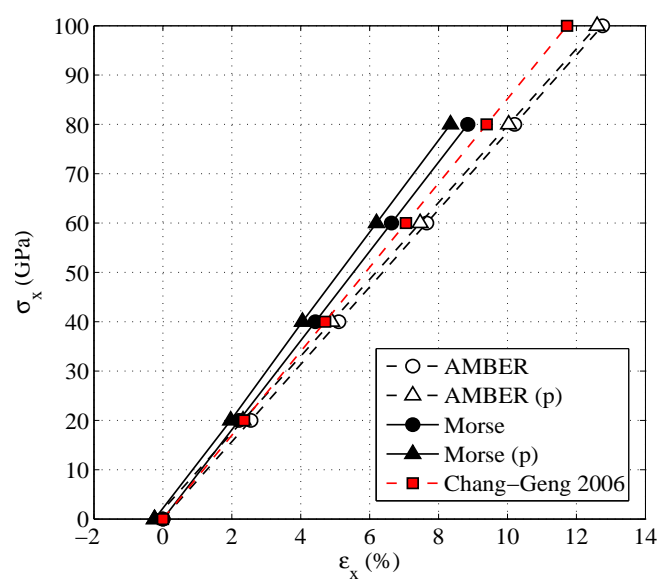

(c) $\mathrm{CH}(10,6)$ tracción

Figura 4.7: SWNTs Chiral a tracción, comparación con [15]

A partir de las figuras 4.6 y 4.7 se puede afirmar que la respuesta longitudinal a tracción es más rígida (en término medio) empleando el potencial de Morse que el de AMBER. A pesar de ello, los resultados obtenidos con ambos potenciales coinciden razonablemente bien para deformaciones longitudinales inferiores al $6 \%$.

Comentario 4.3.1 Conviene señalar que las interrupciones observadas en las curvas tensión deformación de SWNTs Chiral con potencial Morse (fig. 4.7) son debidas a la falta de simetría axial de dichos nanotubos. En particular, al simular estos nanotubos a tracción, algunos enlaces estarán fuertemente traccionados y otros fuertemente comprimidos; teniendo en cuenta que las rigideces tangentes al potencial de Morse difieren mucho de tracción a compresión (fig. 4.1), se produce un mal condicionamiento numérico del proceso iterativo representado en la figura 4.5 para los niveles superiores de tensión.

No se profundizó en las posibles mejoras numéricas del proceso iterativo porque el nivel de deformación en el que aparece la divergencia queda más allá de la hipótesis de pequeños desplaza- 
mientos y por tanto fuera de los objetivos del análisis geométricamente lineal desarrollado en este capítulo.

A pesar de la controversia que implica utilizar algunos parámetros mecánicos intrínsecos de un medio continuo para estudiar el comportamiento mecánico de SWNTs, en parte debido a su fuerte dependencia del espesor de pared equivalente, definimos aquí un módulo de Young equivalente como:

$$
E=\frac{\sigma_{x}}{\epsilon_{x}}
$$

promediando valores para cada curva. De esta forma, seremos capaces de analizar la relación entre el diámetro del CNT y su módulo de Young, con el único objetivo de una comparación cualitativa. Los resultados finales se resumen en la tabla 4.5 y se representan en la figura 4.8

\begin{tabular}{lccccccc} 
& & AMBER & \multicolumn{2}{c}{ AMBER $(\mathrm{p})$} & \multicolumn{2}{c}{ MORSE } & \multicolumn{2}{c}{ MORSE $(\mathrm{p})$} \\
\cline { 2 - 8 } & $\mathrm{d}(\mathrm{nm})$ & $\mathrm{E}(\mathrm{GPa})$ & $\Delta L_{0}(\mathrm{~nm})$ & $\mathrm{E}(\mathrm{GPa})$ & $\mathrm{E}(\mathrm{GPa})$ & $\Delta L_{0}(\mathrm{~nm})$ & $\mathrm{E}(\mathrm{GPa})$ \\
\hline $\mathrm{ZZ}(9,0)$ & 0.7046 & 774.46 & 0.0877 & 774.46 & 897.63 & 0.0888 & 899.52 \\
$\mathrm{ZZ}(11,0)$ & 0.8612 & 783.24 & 0.0705 & 783.16 & 909.09 & 0.0710 & 910.62 \\
$\mathrm{ZZ}(13,0)$ & 1.0178 & 788.67 & 0.0598 & 788.74 & 916.19 & 0.0602 & 917.40 \\
\hline $\mathrm{AC}(5,5)$ & 0.6780 & 786.54 & 0.0430 & 786.56 & 913.83 & 0.0435 & 916.05 \\
$\mathrm{AC}(7,7)$ & 0.9492 & 794.54 & 0.0312 & 794.65 & 924.39 & 0.0312 & 925.88 \\
$\mathrm{AC}(8,8)$ & 1.0848 & 796.77 & 0.0271 & 796.81 & 927.11 & 0.0272 & 928.30 \\
\hline $\mathrm{CH}(7,4)$ & 0.7541 & 778.97 & 0.0454 & 766.43 & 905.94 & 0.0460 & 907.45 \\
$\mathrm{CH}(8,5)$ & 0.8879 & 783.96 & 0.0341 & 775.89 & 900.02 & 0.0344 & 914.84 \\
$\mathrm{CH}(10,6)$ & 1.0960 & 784.06 & 0.0286 & 778.86 & 904.24 & 0.0288 & 925.33 \\
\hline
\end{tabular}

Tabla 4.5: Módulo de Young equivalente a tracción

Para analizar el efecto de las deformaciones iniciales en el comportamiento a tracción, se ensayaron todos los nanotubos con pretensado y en ausencia de cargas externas, observando en la geometría deformada un acortamiento global $\Delta L_{0}(\mathrm{~nm})$ del SWNT como conjunto. Tales acortamientos (en valor absoluto) se incluyen en la tabla 4.5 Este efecto se puede explicar intuitivamente como sigue: las tensiones iniciales introducen fuerzas resultantes en la dirección radial que tienden a mantener la geometría circular de la sección transversal del nanotubo, lo cual se traduce en una tendencia a aumentar el diámetro inicial con el consecuente acortamiento del CNT.

Comentario 4.3.2 Nótese que el origen de deformaciones longitudinales adoptado en los cálculos sin pretensado corresponde al del mapeo conforme, mientras que en los cálculos que incluyen la predeformación corresponde a la posición del extremo libre SWNT tras el acortamiento debido a los esfuerzos iniciales.

Recordemos que durante el proceso de fabricación [26], ya sea en el modo de crecimiento "por la base" o "por la punta"(ver sección 1.1.3), las condiciones reales de sustentación son tipo ménsula. En concreto, cuando un nuevo átomo de carbono se une a un SWNT generado previamente, el conjunto evoluciona hasta una nueva posición de equilibrio, de forma que las tensiones y deformaciones iniciales se introducen paulatinamente en el sistema con cada átomo nuevo. Por tanto, al finalizar el proceso de fabricación y antes de la actuación de las cargas externas, la geometría del nanotubo corresponde con la posterior a la introducción del pretensado (nanotubo "acortado" respecto al mapeo conforme), que constituye el nuevo origen de deformaciones.

Teniendo en cuenta los valores del módulo de Young obtenidos en la tabla 4.5 y las figuras 4.8 , podemos considerar la rigidez longitudinal del nanotubo completamente independiente del pretensado (diferencias máximas relativas $\simeq 2,5 \%$ ) 


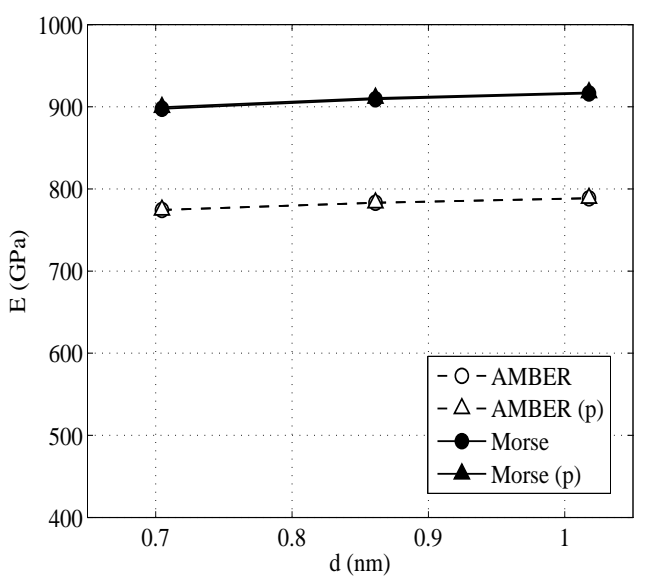

(a) SWNTs ZigZag tracción

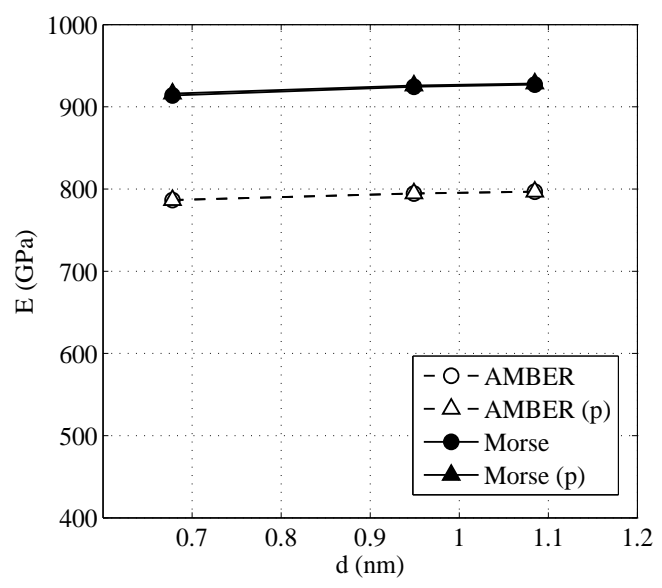

(b) SWNTs Armchair tracción

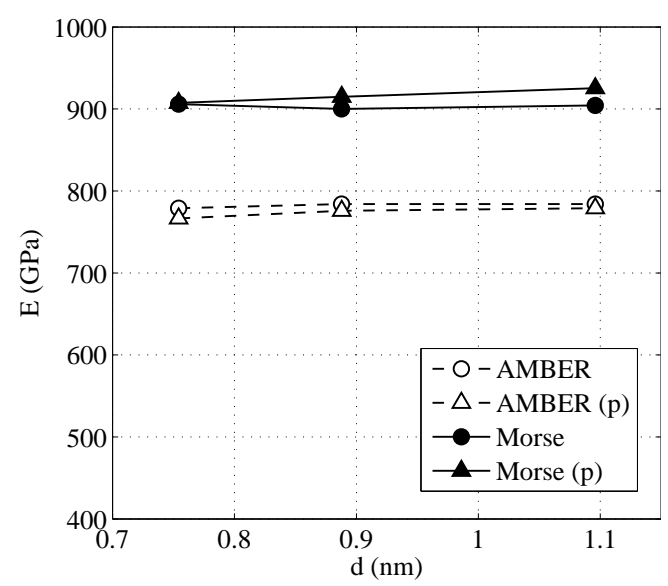

(c) SWNTs Chiral tracción

Figura 4.8: Variación del módulo de Young a tracción con el diámetro

En términos generales, el módulo de Young crece ligeramente con el diámetro del nanotubo, de acuerdo con otros autores ([14, fig 4a], [15], [61, fig 7], [114], [120, fig 12]), tanto considerando la predeformación como sin ella. Aunque esta tendencia es tan somera que se puede considerar $E$ como independiente del diámetro, coincidiendo con [45], [74], [111]. Conviene recordar que algunos autores [14], [37], [44], [88], [114] defienden una tendencia de $E$ creciente con el diámetro hasta un valor asintótico de $E \simeq 1 \mathrm{TPa}$, obtenido experimentalmente para la lámina plana de grafeno.

Para ilustrar la respuesta final de cada tipo de nanotubo a tracción, presentamos en las figuras 4.9 a 4.11 algunos ejemplos de las geometrías deformadas obtenidas mediante la formulación general en rigidez del modelo MSM. Como se puede comprobar (fig.4.11), la falta de axisimetría de los CNTs Chiral hace que se acoplen tracción y torsión, además de producir deformaciones transversales no deseadas en el extremo libre, las cuales pueden distorsionar los resultados finales. Este tipo de deformaciones se pueden evitar imponiendo funciones de desplazamiento conocidas en los nudos

\footnotetext{
${ }^{3}$ dicho comportamiento es tratado analíticamente por [36 mediante un modelo 1D con simetría helicoidal, y es más pronunciado conforme se reduce el diámetro
} 


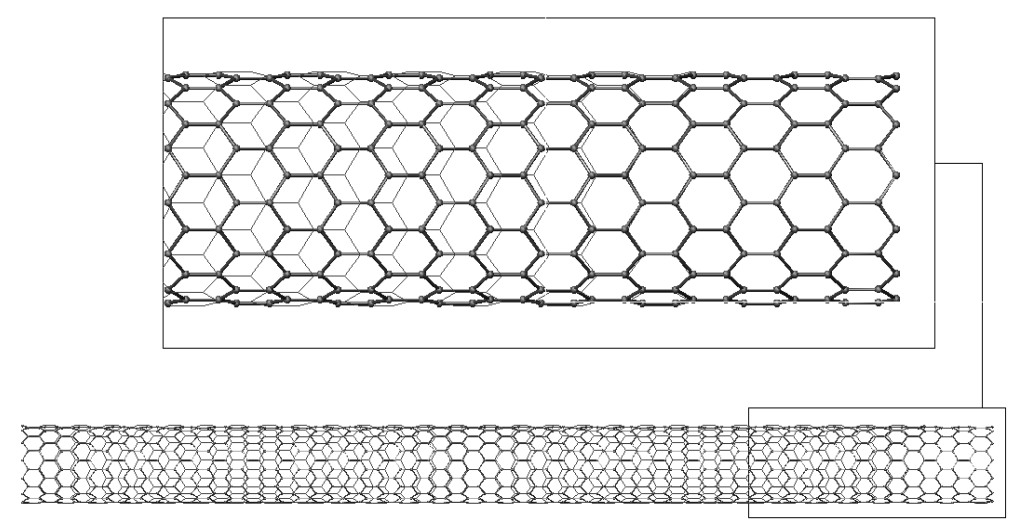

Figura 4.9: Deformada ZZ(13,0) $\sigma_{x}=60 \mathrm{GPa}, \mathrm{AMBER}$

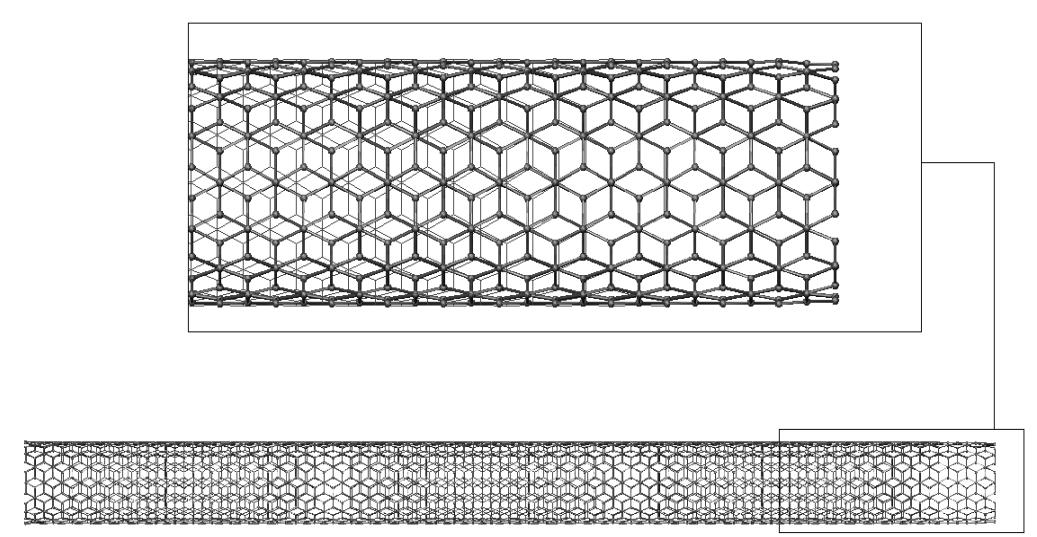

Figura 4.10: Deformada $A C(8,8) \sigma_{x}=60 \mathrm{GPa}$, Morse (p)

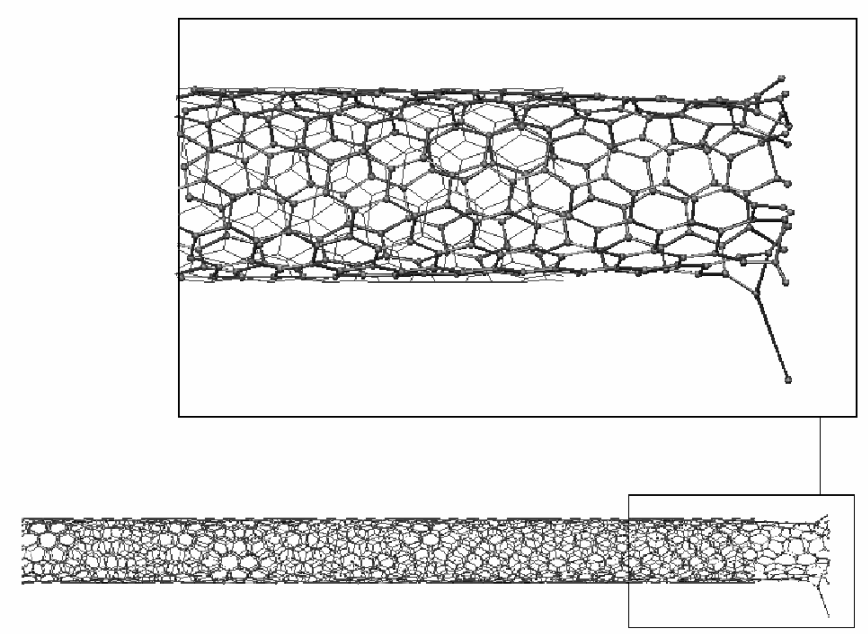

Figura 4.11: Deformada $C H(8,5) \sigma_{x}=80 \mathrm{GPa}$, AMBER 
del extremo derecho, de forma que los átomos asociados se mantengan en la sección transversal circular.

Teniendo en cuenta que estas deformaciones transversales sólo son de magnitud considerable para los niveles superiores de tensión y que incluso en estos casos no pasan de ser un efecto local, su tratamiento mediante una corona de apoyos fijos en el extremo derecho dotados de un sistema de desplazamientos impuestos se ha postergado al capítulo [5, donde se desarrolla la extensión geométricamente no lineal de la formulación. En caso de no adoptar dicho sistema de desplazamientos impuestos en un análisis no lineal geométrico, los modos de inestabilidad clásicos quedarían completamente desvirtuados.

Con el objetivo de deducir la influencia de la quiralidad en el comportamiento longitudinal a tracción de SWNTs, se ensayan dos nanotubos adicionales (tabla 4.6) con potencial AMBER incluyendo el pretensado, y se comparan con los correspondientes al diámetro intermedio de cada quiralidad de los definidos en la tabla 4.3. También se han incluido en la tabla 4.6 los acortamientos del pretensado en ausencia de cargas externas. Los resultados se resumen en la tabla 4.7 y se representan en la figura 4.12

\begin{tabular}{lcccc}
\hline Quiralidad & $\mathrm{d}(\mathrm{nm})$ & $\mathrm{L}(\mathrm{nm})$ & $\mathrm{L} / \mathrm{d}$ & $\Delta L_{0}(\mathrm{~nm})$ \\
\hline $\mathrm{CH}(8,4)$ & 0.8285 & 10.1436 & 12.2 & 0.04661 \\
$\mathrm{CH}(10,4)$ & 0.9707 & 11.5281 & 11.9 & 0.04286 \\
\hline
\end{tabular}

Tabla 4.6: Dimensiones y quiralidades de los SWNTs adicionales a tracción

\begin{tabular}{|c|c|c|c|c|c|}
\hline & $\mathrm{d}(\mathrm{nm})$ & $\mathrm{L}(\mathrm{nm})$ & $\mathrm{L} / \mathrm{d}$ & $\theta\left({ }^{\circ}\right)$ & $\mathrm{E}(\mathrm{GPa})$ \\
\hline$\overline{Z Z}(11,0)$ & 0.8612 & 10.2950 & 12.0 & 0.0 & 783.16 \\
\hline $\mathrm{CH}(10,4)$ & 0.9707 & 11.5281 & 11.9 & 16.1 & 778.09 \\
\hline $\mathrm{CH}(8,4)$ & 0.8285 & 10.1436 & 12.2 & 19.1 & 771.54 \\
\hline $\mathrm{CH}(8,5)$ & 0.8879 & 9.6767 & 10.9 & 22.4 & 775.89 \\
\hline $\operatorname{AC}(7,7)$ & 0.9492 & 11.3138 & 11.9 & 30.0 & 794.65 \\
\hline
\end{tabular}

Tabla 4.7: Módulo de Young a tracción frente a quiralidad

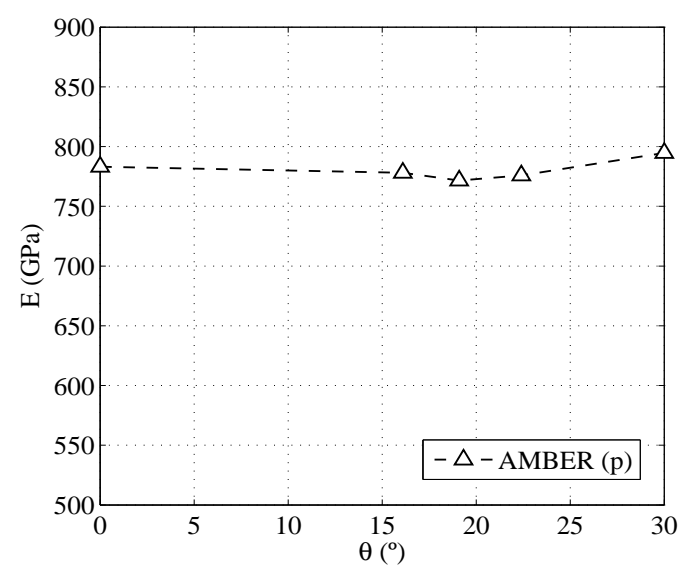

Figura 4.12: Influencia de la quiralidad en el módulo de Young a tracción

A partir de estos resultados, se puede deducir que la rigidez longitudinal de los SWNTs es prácticamente independiente de la quiralidad (figura 4.12). Incluso algunos autores [74], [111] defienden esta independencia bajo una relación constitutiva no lineal (potenciales Morse o T-B). 


\subsubsection{Comportamiento a compresión}

Se estudia a continuación el comportamiento a compresión de SWNTs adoptando las mismas condiciones de sustentación (tipo ménsula) y análogas condiciones de carga. En este caso se adoptan tensiones de 30,60, $120 \mathrm{GPa}$ calculadas mediante la ec. (4.31), aplicadas mediante cargas puntuales longitudinales de valor constante $R_{i}=F_{t} / N_{t}$ en los nudos del extremo libre. Estos valores se resumen en la tabla 4.8 .

\begin{tabular}{|c|c|c|c|c|c|c|}
\hline & $R_{i}(\mathrm{nN})$ & $\overline{F_{t}(\mathrm{nN})}$ & $\overline{R_{i}(\mathrm{nN})}$ & $F_{t}(\mathrm{nN})$ & $\overline{R_{i}(\mathrm{nN})}$ & $\bar{F}(\mathrm{nN})$ \\
\hline$\sigma_{x}(\mathrm{Gpa})$ & \multicolumn{2}{|c|}{$\mathrm{ZZ}(9,0)$} & \multicolumn{2}{|c|}{$\mathrm{ZZ}(11,0)$} & \multicolumn{2}{|c|}{$\mathrm{ZZ}(13,0)$} \\
\hline 30 & 2.51 & 22.58 & 2.51 & 27.60 & 2.51 & 32.61 \\
\hline 60 & 5.02 & 45.16 & 5.02 & 55.19 & 5.02 & 65.23 \\
\hline 120 & 10.03 & 90.31 & 10.03 & 110.38 & 10.03 & 130.45 \\
\hline$\sigma_{x}(\mathrm{Gpa})$ & \multicolumn{2}{|c|}{$\mathrm{AC}(5,5)$} & \multicolumn{2}{|c|}{$\mathrm{AC}(7,7)$} & \multicolumn{2}{|c|}{$\mathrm{AC}(8,8)$} \\
\hline 30 & 2.17 & 21.73 & 2.17 & 30.42 & 2.17 & 34.76 \\
\hline 60 & 4.35 & 43.45 & 4.35 & 60.83 & 4.35 & 69.52 \\
\hline 120 & 8.69 & 86.90 & 8.69 & 121.67 & 8.69 & 139.05 \\
\hline$\sigma_{x}(\mathrm{Gpa})$ & \multicolumn{2}{|c|}{$\mathrm{CH}(7,4)$} & \multicolumn{2}{|c|}{$\mathrm{CH}(8,5)$} & \multicolumn{2}{|c|}{$\mathrm{CH}(10,6)$} \\
\hline 30 & 2.20 & 24.17 & 2.19 & 28.45 & 2.20 & 35.12 \\
\hline 60 & 4.39 & 48.33 & 4.38 & 56.90 & 4.39 & 70.24 \\
\hline 120 & 8.79 & 96.66 & 8.75 & 113.80 & 8.78 & 140.49 \\
\hline
\end{tabular}

Tabla 4.8: Cargas puntuales aplicadas en simulaciones a compresión

Se representan en las figuras 4.13 y 4.14las curvas tensión-deformación obtenidas a compresión. Análogamente a lo expuesto a tracción, comparamos los resultados obtenidos para los SWNTs ZigZag (ZZ) y Armchair (AC) con los de Natsuki et. al. [78] procesados con el potencial AMBER, y con los de Natsuki y Endo [77] procesados con el potencial Morse. En ninguno de las dos referencias se incluye el pretensado. Por otra parte, para los SWNTs Chiral $(\mathrm{CH})$ se reproducen los resultados de Ghang et. al. [15], aunque con las constantes del AMBER.

Como puede comprobarse, nuestros resultados están en concordancia con los obtenidos en las referencias empleadas, sobre todo para nanotubos AC. No obstante, se observan las mayores diferencias para nanotubos $\mathrm{ZZ}$ con el potencial Morse debido a que nuestro procedimiento numérico trabaja con rigideces tangentes sobre la rama de compresión de la función potencial (fig 4.1(a)], frente a Natsuki y Endo [77] que incluyen las relaciones constitutivas exactas. Esta aproximación, unida a que un tercio de los enlaces son axiales en SWNTS ZigZag, produce un comportamiento algo más flexible a compresión.

En todos los casos el potencial Morse produce resultados más rígidos a compresión que el AM$\mathrm{BER}$, a causa de la mayor pendiente del primero en la rama de compresión frente al alargamiento de enlace. De hecho, la diferencia se hace patente para deformaciones longitudinales del SWNT alrededor del $6 \%$.

Las interrupciones observadas en las curvas de la figura 4.14 para SWNTs Chiral se deben a la falta de axisimetría de estos nanotubos y han sido explicadas en el comentario 4.3.1

Comentario 4.3.3 La validez de los resultados y comentarios expuestos aquí se limitan a deformaciones longitudinales inferiores a las críticas. Puesto que nuestro análisis hasta el momento es geométricamente lineal, la obtención de dichas deformaciones queda fuera de los objetivos del presente capítulo y se ha desarrollado detalladamente en el capítulo 5. Sin embargo, las conclusiones extraídas del análisis lineal tienen un valor cualitativo como primer paso para comprender el comportamiento longitudinal de SWNTs y sirven para la comparación con la respuesta a tracción. 


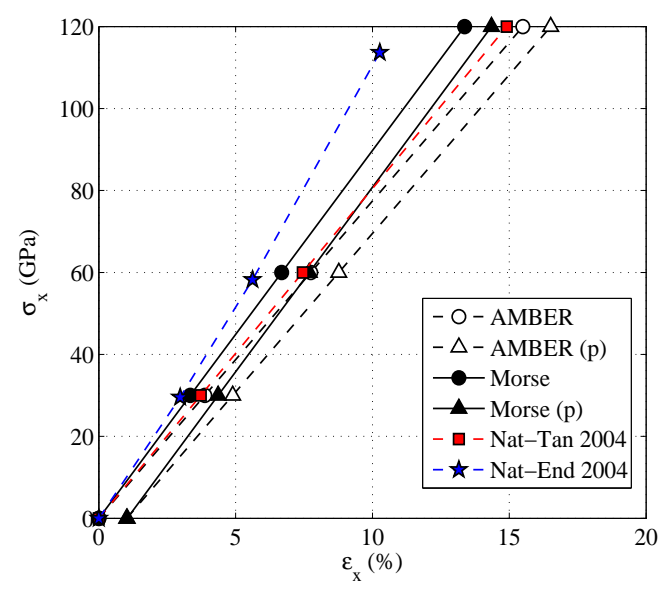

(a) $\mathrm{ZZ}(9,0)$ compresión

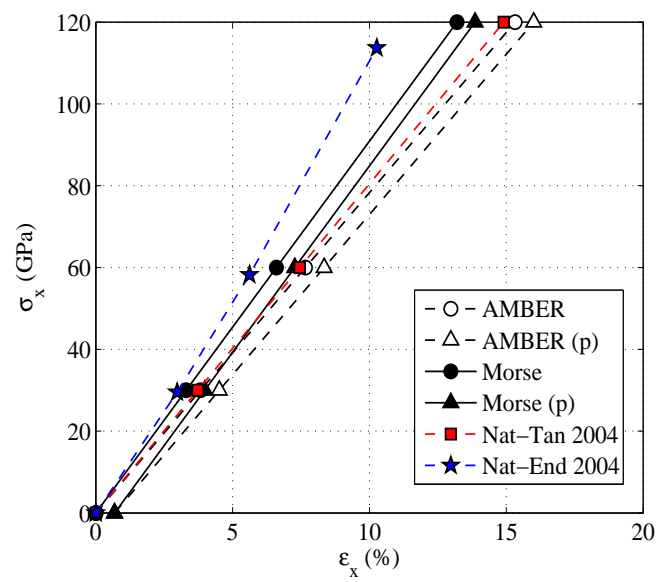

(c) ZZ(11,0) compresión

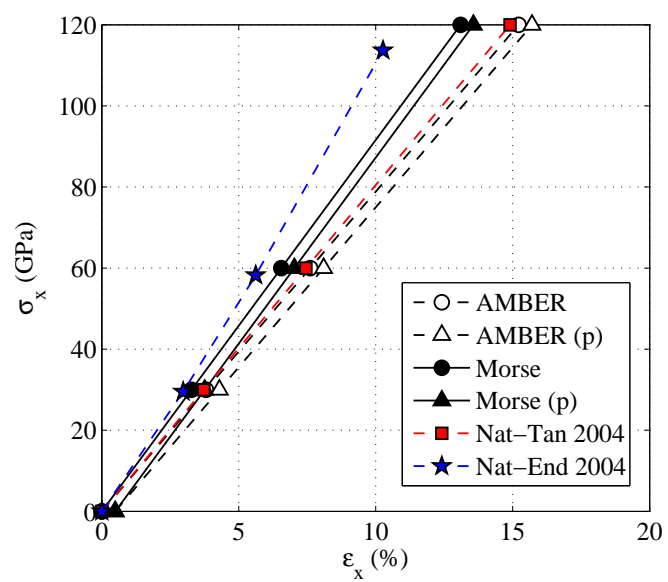

(e) ZZ(13,0) compresión

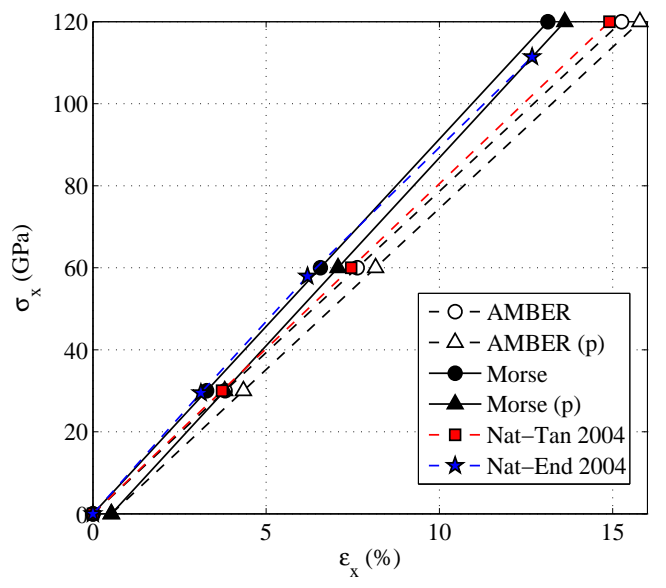

(b) $\mathrm{AC}(5,5)$ compresión

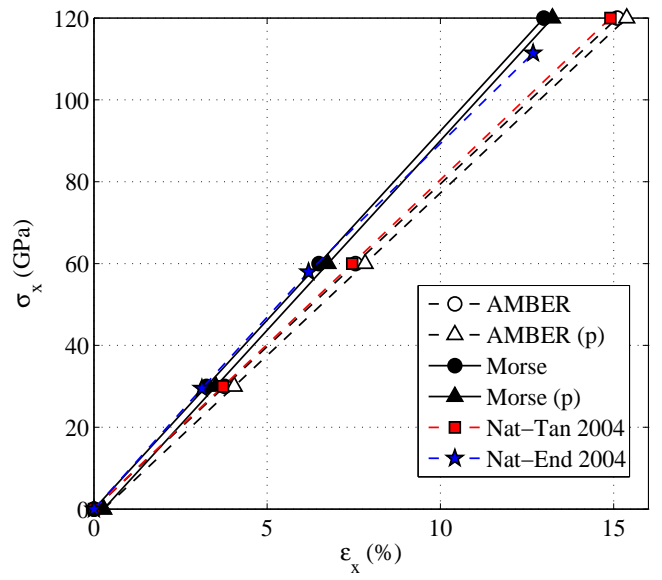

(d) $\mathrm{AC}(7,7)$ compresión

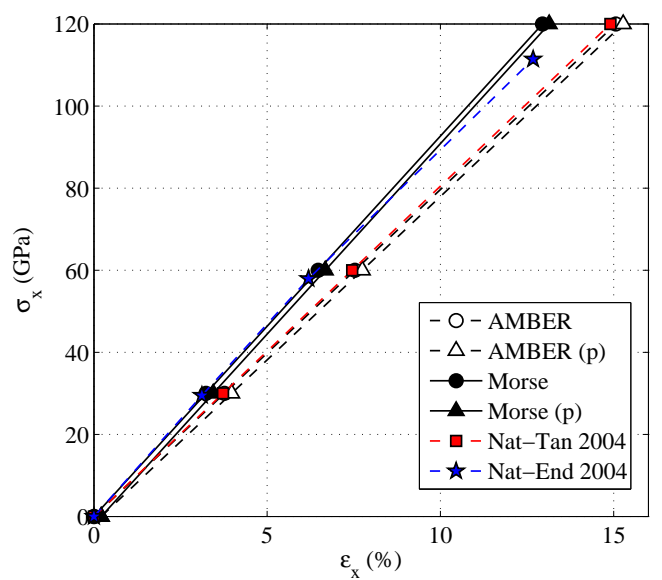

(f) $\mathrm{AC}(8,8)$ compresión

Figura 4.13: SWNTs Armchair y ZigZag a compresión, comparación con [77] y [78] 


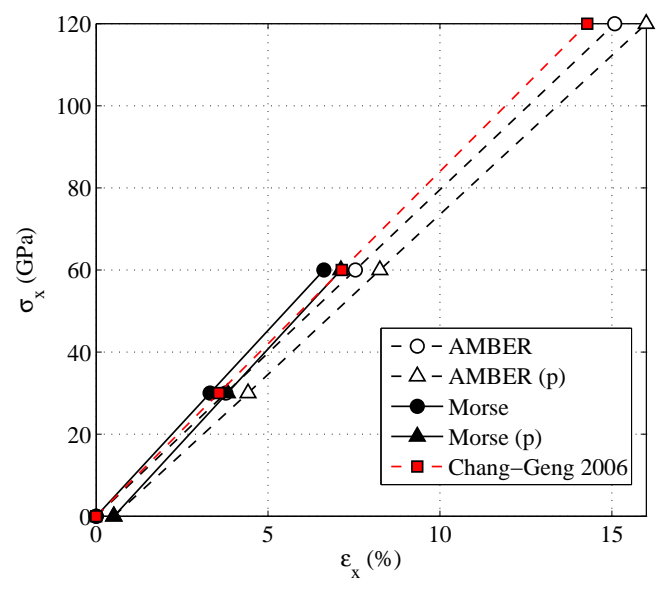

(a) $\mathrm{CH}(7,4)$ compresión

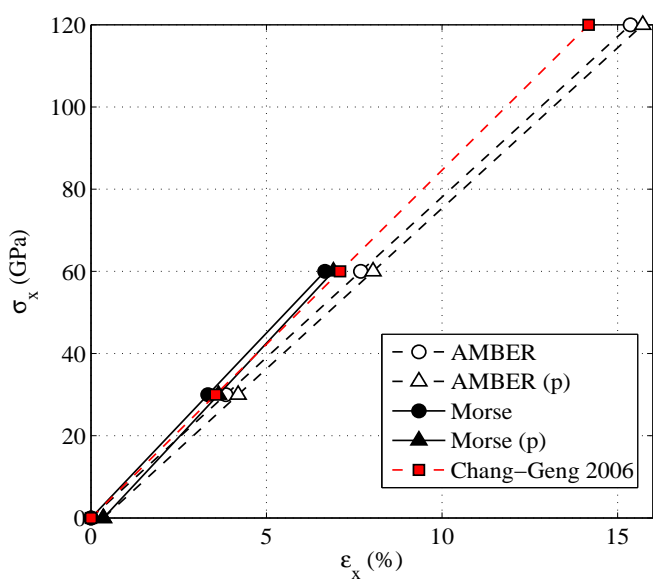

(b) $\mathrm{CH}(8,5)$ compresión

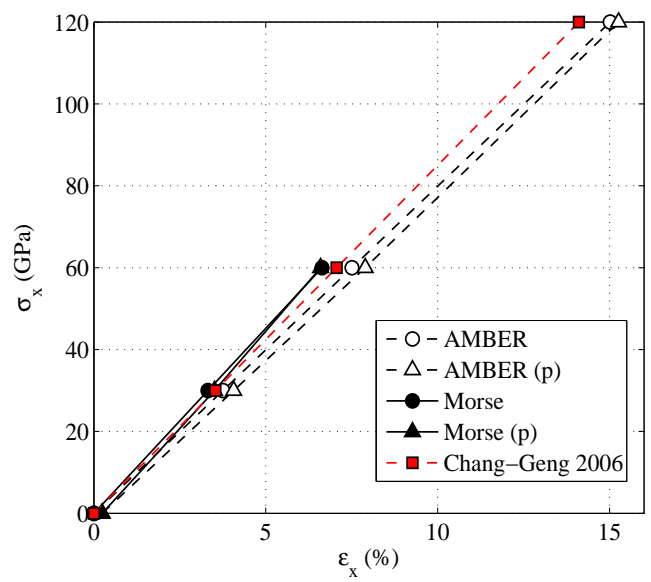

(c) $\mathrm{CH}(10,6)$ compresión

Figura 4.14: SWNTs Chiral a compresión, comparación con [15]

Aceptando la definición (4.32) para el módulo de Young a compresión y promediando valores en cada curva, podemos representar la variación de dicho parámetro frente al diámetro en la figura 4.15 y resumir los valores obtenidos en la tabla 4.9

Se observa una ligera tendencia creciente en el módulo de Young, tanto si incluimos el pretensado en el análisis como si no. Sin embargo, esta tendencia es tan somera que en el rango de diámetros simulados lo podemos considerar prácticamente constante.

A partir de los valores de la tabla 4.9 la quiralidad no tiene un efecto relevante en la respuesta a compresión de SWNTs y puede ser despreciado, tal como se concluyó a tracción. Asimismo, la influencia del pretensado puede considerarse despreciable a compresión (diferencias relativas máximas $\simeq 3 \%$ ).

Evidentemente, si consideramos el potencial de Morse, se obtienen diferentes módulos de Young entre tracción y compresión. Por tanto, la aplicabilidad de modelos continuos resulta dudosa, como ha sido puesto de relieve en algunos trabajos publicados [14], [114]. 


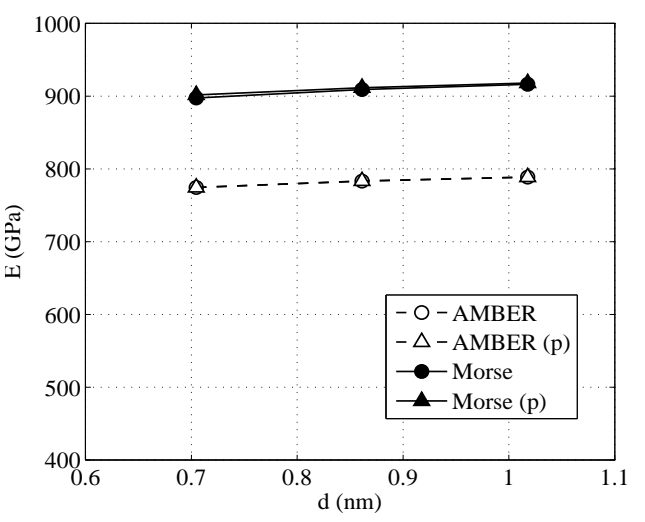

(a) SWNTs ZigZag compresión

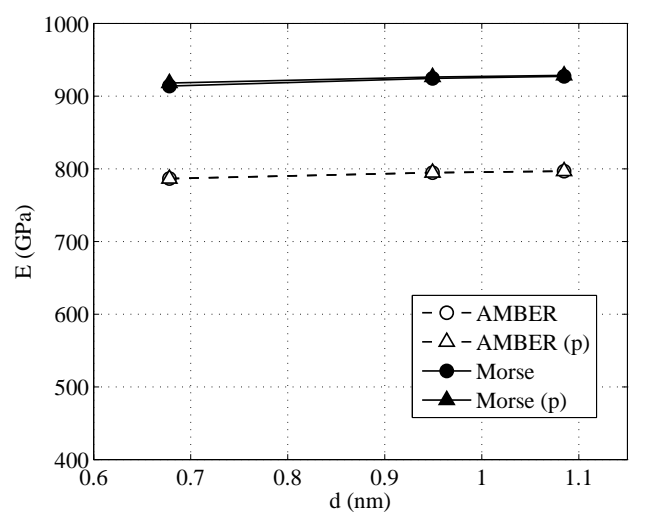

(b) SWNTs Armchair compresión

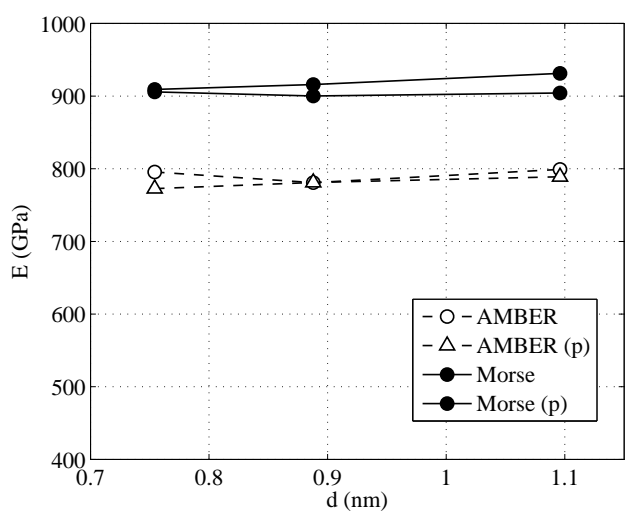

(c) SWNTs Chiral compresión

Figura 4.15: Variación del Módulo de Young a compresión con el diámetro

\begin{tabular}{lccccc}
\cline { 2 - 6 } & & AMBER & AMBER $(\mathrm{p})$ & MORSE & MORSE $(\mathrm{p})$ \\
\cline { 2 - 6 } & $\mathrm{d}(\mathrm{nm})$ & $\mathrm{E}(\mathrm{GPa})$ & $\mathrm{E}(\mathrm{GPa})$ & $\mathrm{E}(\mathrm{GPa})$ & $\mathrm{E}(\mathrm{GPa})$ \\
\hline $\mathrm{ZZ}(9,0)$ & 0.7046 & 774.41 & 774.41 & 897.52 & 901.59 \\
$\mathrm{ZZ}(11,0)$ & 0.8612 & 783.21 & 783.28 & 909.07 & 911.60 \\
$\mathrm{ZZ}(13,0)$ & 1.0178 & 788.68 & 788.65 & 916.24 & 917.96 \\
\hline $\mathrm{AC}(5,5)$ & 0.6780 & 786.48 & 786.43 & 913.81 & 917.98 \\
$\mathrm{AC}(7,7)$ & 0.9492 & 794.66 & 794.64 & 924.39 & 926.21 \\
$\mathrm{AC}(8,8)$ & 1.0848 & 796.78 & 796.75 & 927.13 & 928.55 \\
\hline $\mathrm{CH}(7,4)$ & 0.7541 & 795.56 & 772.58 & 905.87 & 909.17 \\
$\mathrm{CH}(8,5)$ & 0.8879 & 780.97 & 781.08 & 900.15 & 915.90 \\
$\mathrm{CH}(10,6)$ & 1.0960 & 799.16 & 788.96 & 904.26 & 931.34 \\
\hline
\end{tabular}

Tabla 4.9: Módulo de Young equivalente a compresión

A modo de ejemplo, representamos en las figuras 4.16 a 4.18 algunas geometrías deformadas a compresión. En la figura 4.18 se puede observar para SWNTs Chiral el mismo fenómeno de deformación local no deseada en el extremo libre que a tracción. 


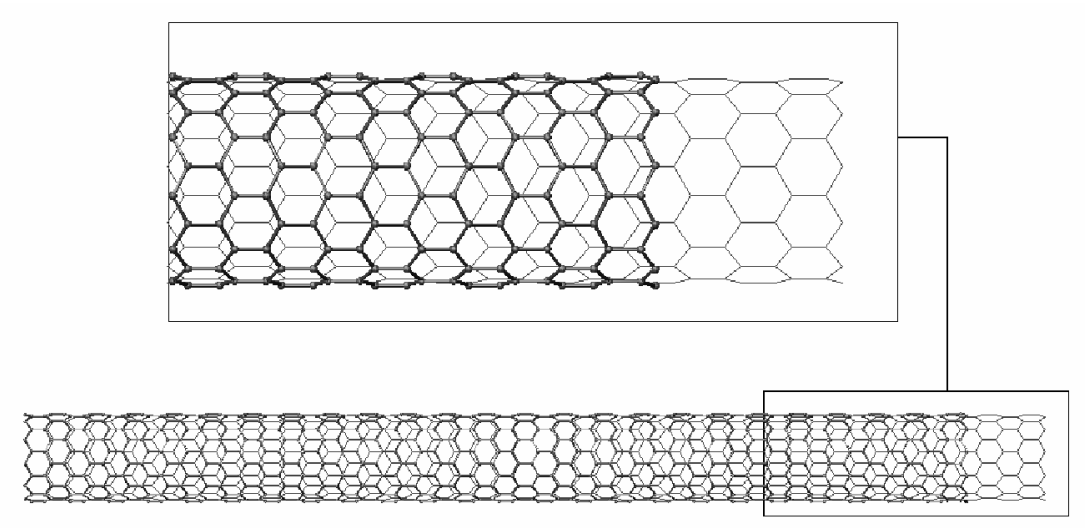

Figura 4.16: Deformada ZZ(11,0) $\sigma_{x}=60 \mathrm{GPa}, \mathrm{AMBER}$

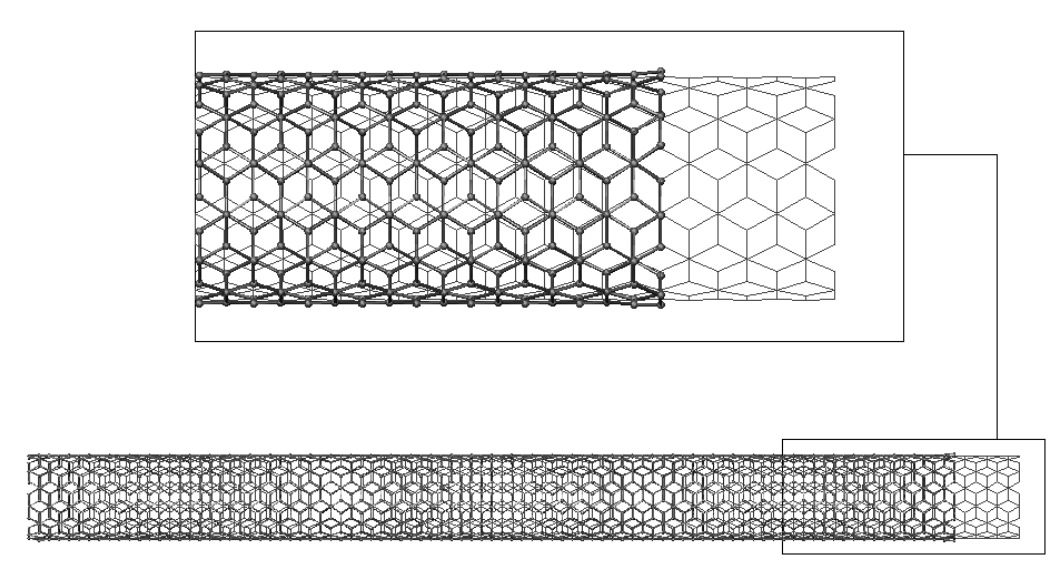

Figura 4.17: Deformada $A C(7,7) \sigma_{x}=60 \mathrm{GPa}$, Morse
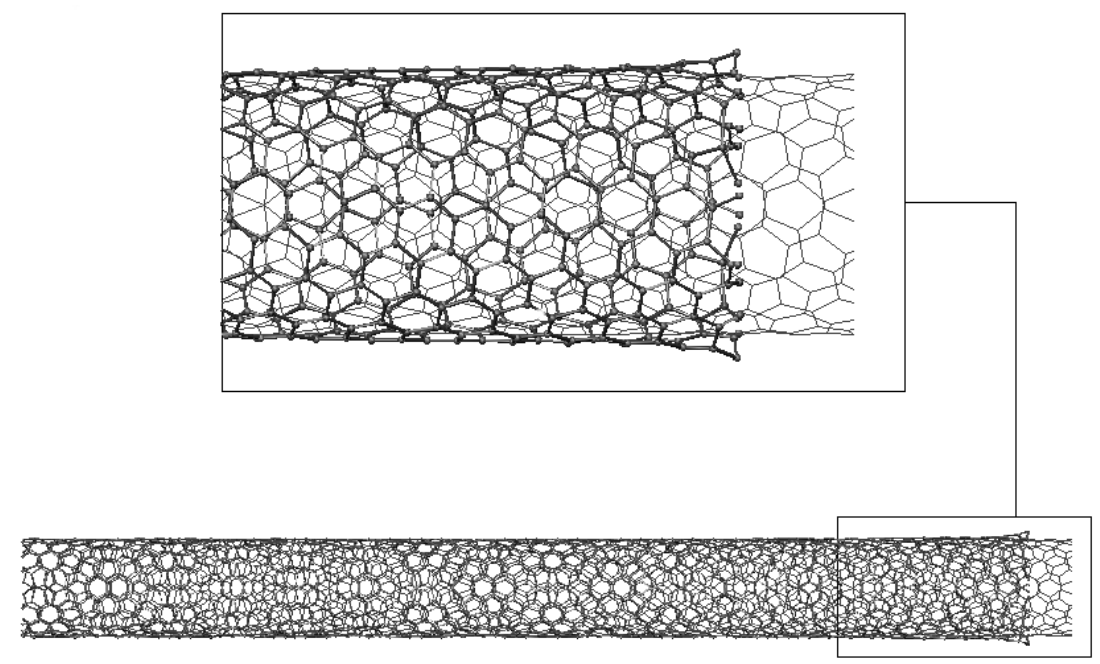

Figura 4.18: Deformada $C H(10,6) \sigma_{x}=30 \mathrm{GPa}, \mathrm{AMBER}(\mathrm{p})$ 


\subsubsection{Comportamiento a flexión}

La respuesta a flexión de SWNTs aislados sigue siendo hasta la fecha un problema sólo tratado lateralmente [17], [50], [99] en la literatura. En cambio, la versatilidad de la presente formulación frente a casos generales de carga permite tratarlo de forma directa. Para este propósito, se mantienen las condiciones de sustentación tipo ménsula (mediante apoyos fijos en el extremo izquierdo) y se aplica en el extremo libre un momento flector externo con los valores de la tabla 4.10.

\begin{tabular}{cccc}
\cline { 2 - 4 } & $\mathrm{ZZ}(9,0)$ & $\mathrm{ZZ}(11,0)$ & $\mathrm{ZZ}(13,0)$ \\
& $\mathrm{AC}(5,5)$ & $\mathrm{AC}(7,7)$ & $\mathrm{AC}(8,8)$ \\
& $\mathrm{CH}(7,4)$ & $\mathrm{CH}(8,5)$ & $\mathrm{CH}(10,6)$ \\
\hline \multirow{3}{*}{$\mathrm{M}(\mathrm{nN} \cdot \mathrm{nm})$} & 0.5 & 1.0 & 1.0 \\
& 1.0 & 2.0 & 2.0 \\
& 2.0 & 3.0 & 4.0 \\
\hline
\end{tabular}

Tabla 4.10: Momentos externos de flexión $(\mathrm{nN} \cdot \mathrm{nm})$ aplicados en el extremo libre

Cada momento externo se ha convertido en un sistema estáticamente equivalente de fuerzas puntuales longitudinales en los nudos del extremo libre. Puesto que este sistema no es único, será necesario adoptar alguna hipótesis adicional de variación para sus valores. En este caso, si definimos el eje $\{z\}$ como el eje de flexión y el $\{y\}$ como el eje ortogonal en la sección transversal del nanotubo, supondremos que los valores de las cargas puntuales siguen una variación lineal con la coordenada $\{y\}$, de la forma:

$$
R_{x i}=\frac{M_{z}}{\sum y_{i}^{2}} y_{i}
$$

Como ejemplo, se incluyen en la tabla 4.11 algunos valores numéricos de estas cargas puntuales bajo un momento de $M_{z}=1 \mathrm{nN} \cdot \mathrm{nm}$, donde los valores positivos indican fuerzas de tracción y los negativos de compresión.

\begin{tabular}{cccccc}
\hline \multicolumn{2}{c}{$\mathrm{ZZ}(11,0)$} & \multicolumn{2}{c}{$\mathrm{AC}(5,5)$} & \multicolumn{2}{c}{$\mathrm{CH}(7,4)$} \\
\hline$y_{i}(\mathrm{~nm})$ & $R_{x i}(\mathrm{nN})$ & $y_{i}(\mathrm{~nm})$ & $R_{x i}(\mathrm{nN})$ & $y_{i}(\mathrm{~nm})$ & $R_{x i}(\mathrm{nN})$ \\
\hline 0.4131 & 0.41 & 0.3390 & 0.59 & 0.3693 & 0.47 \\
0.4131 & 0.41 & 0.3097 & 0.54 & 0.3547 & 0.45 \\
0.2820 & 0.28 & 0.2268 & 0.39 & 0.2892 & 0.37 \\
0.2820 & 0.28 & 0.1048 & 0.18 & 0.1888 & 0.24 \\
0.0613 & 0.06 & 0.1048 & 0.18 & 0.0655 & 0.08 \\
0.0613 & 0.06 & -0.0354 & -0.06 & 0.0394 & 0.05 \\
-0.1789 & -0.18 & -0.1354 & -0.29 & -0.0117 & -0.15 \\
-0.1789 & -0.18 & -0.2743 & -0.48 & -0.1887 & -0.24 \\
-0.3622 & -0.36 & -0.2743 & -0.48 & -0.2892 & -0.37 \\
-0.3622 & -0.36 & -0.3316 & -0.58 & -0.3449 & -0.44 \\
-0.4306 & -0.42 & & & -0.3775 & -0.48 \\
\hline
\end{tabular}

Con el objetivo de investigar cualitativamente la respuesta a flexión de SWNTs, se estiman las rotaciones del extremo libre (concepto asociado a una viga) para representarlas frente al momento externo aplicado (ver figs. 4.19 y 4.20). Dicha estimación se ha efectuado proyectando el cambio de orientación de una recta ficticia que une dos átomos diametralmente opuestos del extremo libre, sobre el plano vertical que contiene al eje del nanotubo. 


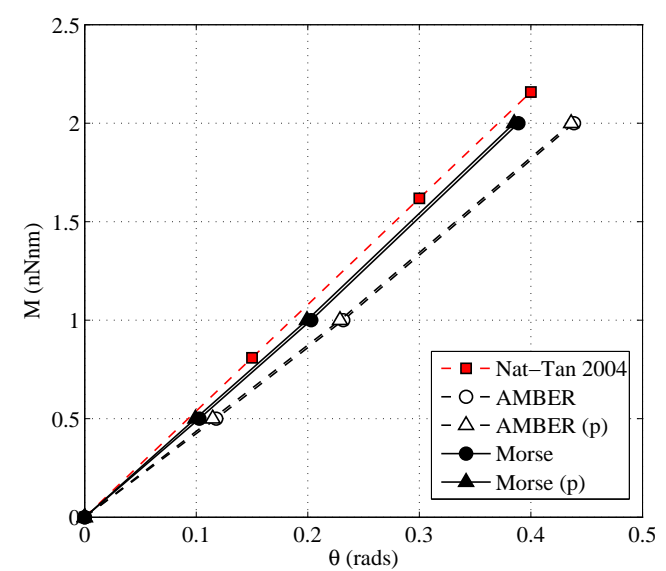

(a) $\mathrm{ZZ}(9,0)$ flexión

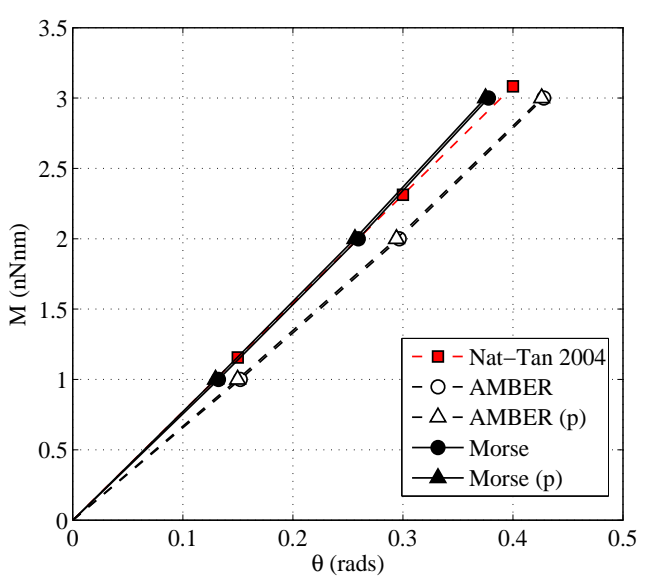

(c) $\mathrm{ZZ}(11,0)$ flexión

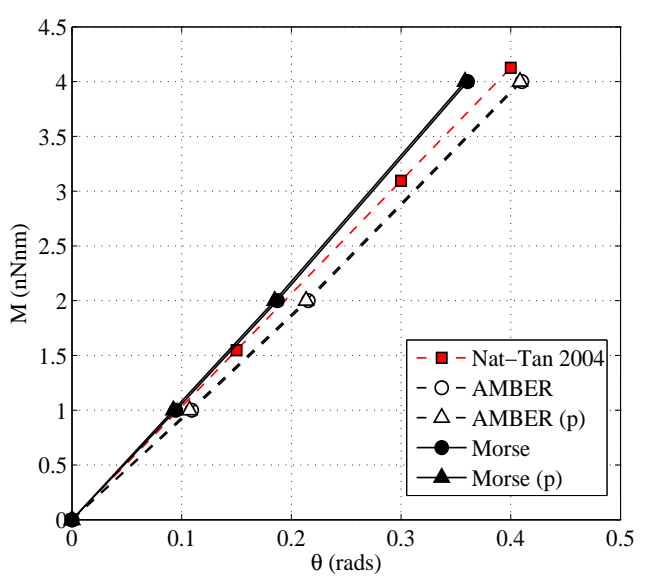

(e) $\mathrm{ZZ}(13,0)$ flexión

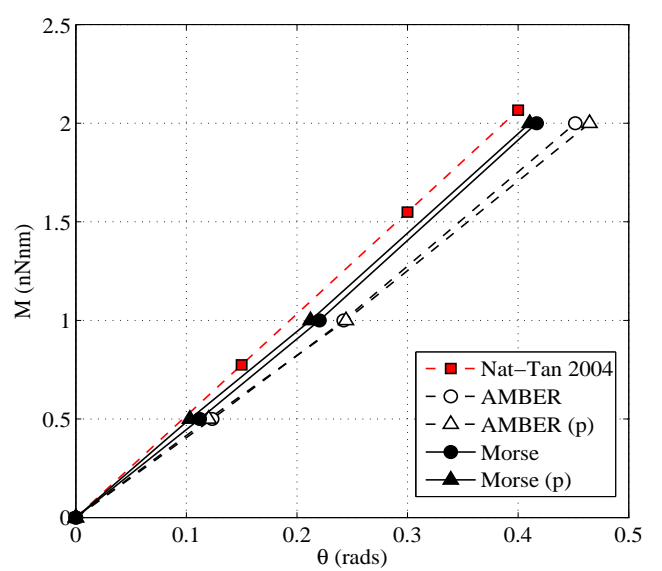

(b) $\mathrm{AC}(5,5)$ flexión

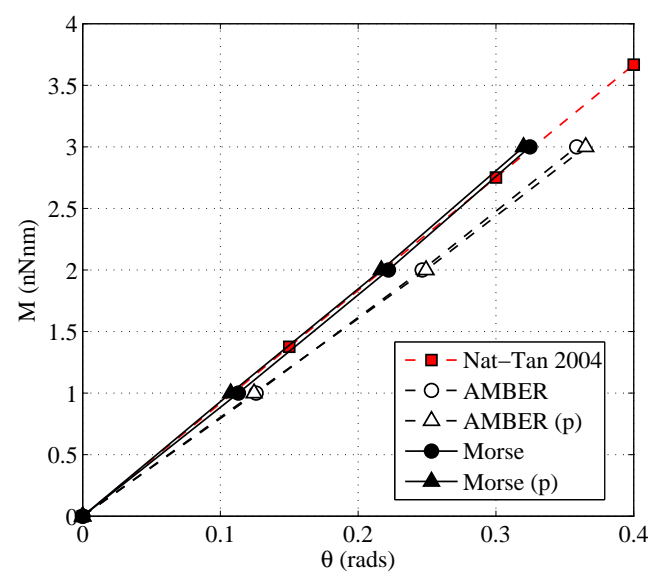

(d) $\mathrm{AC}(7,7)$ flexión

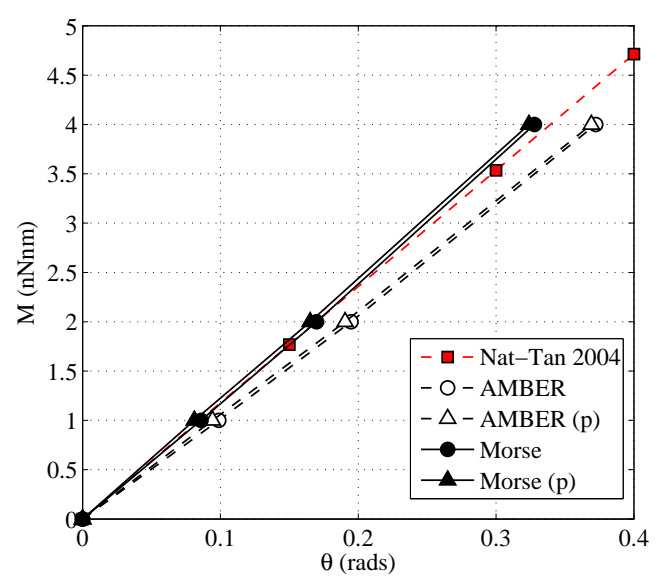

(f) $\mathrm{AC}(8,8)$ flexión

Figura 4.19: SWNTs Armchair y ZigZag a flexión, comparación con [78] 


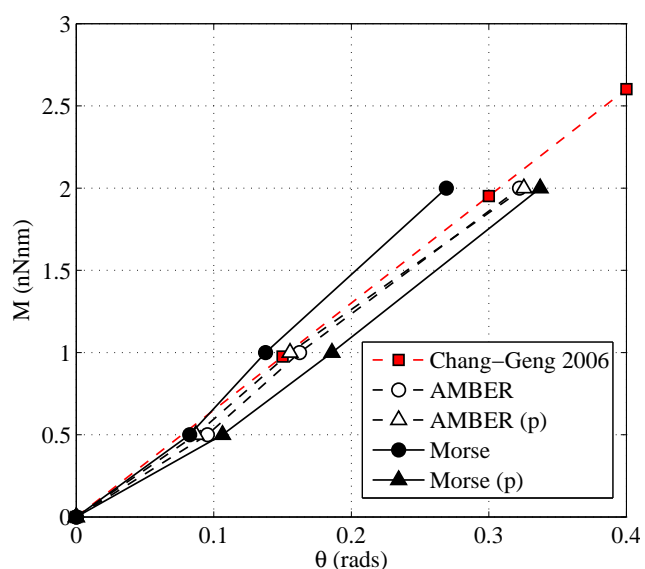

(a) $\mathrm{CH}(7,4)$ flexión

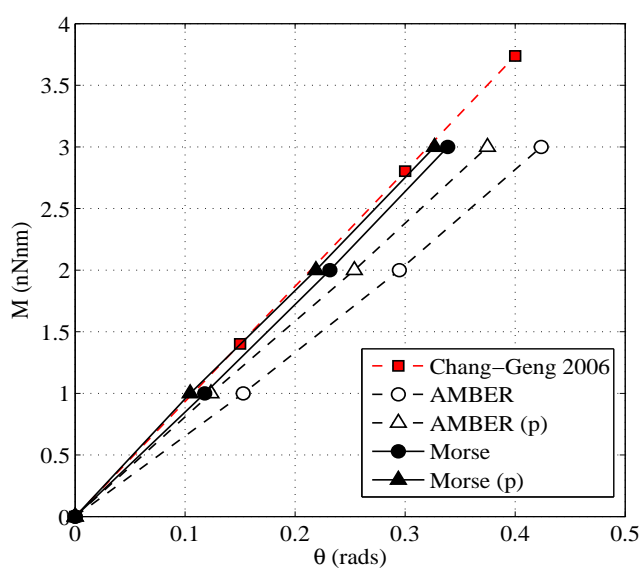

(b) $\mathrm{CH}(8,5)$ flexión

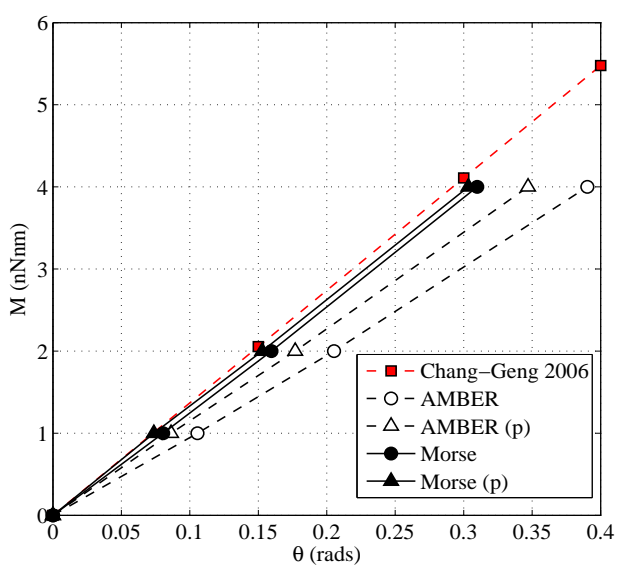

(c) $\mathrm{CH}(10,6)$ flexión

Figura 4.20: SWNTs Chiral a flexión, comparación con 15]

Todas las curvas muestran un comportamiento sensiblemente lineal incluso empleando el potencial de Morse4. Por tanto, la influencia de la interacción no lineal entre átomos debida a la función potencial tiene muy poca influencia en la respuesta a flexión, como cabía esperar.

Por otra parte, la influencia del pretensado puede ser despreciada en SWNTs ZigZag y Armchair ya que apenas provoca diferencias en la respuesta final. En nanotubos axisimétricos, el efecto de acortamiento inicial introducido por el pretensado (relacionado con la respuesta a axil) está entonces desacoplado de la respuesta a flexión. Sin embargo, en los SWNTs Chiral el comportamiento longitudinal y de flexión están acoplados por la configuración helicoidal de estos nanotubos, dando lugar a un efecto rigidizador de las tensiones iniciales que se refleja en una mayor pendiente de las curvas $M-\theta$ que incluyen el pretensado (figura 4.20).

Cabe señalar que no procede modificar del origen de deformaciones longitudinales en los análisis con pretensado (Comentario 4.3.2) a flexión, ya que se ha adoptado la rotación de la sección extre-

\footnotetext{
${ }^{4}$ Los quiebros presentes en la figura 4.20(a) se deben a errores causados por la dificultad en la estimación de la rotación del extremo libre para SWNTs Chiral de reducido diámetro
} 
ma como deformación representativa y el efecto del pretensado correspondería con una traslación longitudinal al SWNT de dicha sección.

Puesto que la simulación de SWNTs a flexión mediante modelos de mecánica molecular es un tema no tratado en las referencias consultadas, la comparación de las curvas obtenidas $M-\theta$ con la literatura es complicada. Sin embargo, se puede estimar la rigidez a flexión de los SWNTs (en orden de magnitud) mediante la ecuación clásica de teoría de vigas:

$$
M_{z}=\frac{E I}{L} \theta
$$

donde $L$ es la longitud inicial del nanotubo e $I$ es el momento de inercia de la sección transversal considerada como un anillo circular de espesor $t=0.34 \mathrm{~nm}$. Adoptando para los nanotubos AC y ZZ un valor de $E=805.01 \mathrm{GPa}$ extraído de [78], y para los nanotubos Chiral los valores de [15] $(E=$ 840.23 GPa para el $\mathrm{CH}(7,4), E=843.77 \mathrm{GPa}$ para el $\mathrm{CH}(8,5)$ y $E=847.64 \mathrm{GPa}$ para el $\mathrm{CH}(10,6))$, se obtienen las curvas $M-\theta$ asociadas a las citadas referencias en las figuras 4.19 y 4.20 .

Como se puede comprobar, extrapolar los valores del módulo de Young a tracción para estimar una rigidez a flexión equivalente produce resultados alrededor de un $23 \%$ más rígidos que los obtenidos con simulaciones directas a flexión mediante el modelo atómico MSM.

Comentario 4.3.4 Algunas curvas $M-\theta$ de SWNTs Chiral presentan ligeros quiebros que se desvían del comportamiento lineal (fig. 4.20). Éstos vienen motivados por la dificultad en la estimación del giro en el extremo libre causada por las deformaciones locales mencionadas a tracción y compresión, presentes también a flexión.

Por otra parte, extendemos al caso de flexión las ideas expuestas en el comentario 4.3.3.

En virtud de una posterior comparación entre los parámetros mecánicos obtenidos a tracción/compresión y los obtenidos directamente a flexión, calculamos el módulo de Young equivalente para este último caso a partir de la ec. (4.34) como:

$$
E=\frac{M L}{I \theta}
$$

Promediando valores para cada CNT, se obtienen los valores de la tabla 4.12 Estos resultados presentan una dispersión considerable y ponen de manifiesto que los modelos continuos (y en particular la teoría clásica de vigas) no son aplicables a escala "nano" debido a que la relativamente pequeña cantidad de átomos implicados impide el promedio de propiedades mecánicas. Esta idea está en claro desacuerdo con varias referencias consultadas (por ejemplo, [17], [50], [93], [112], [118], [119]).

\begin{tabular}{lccccc}
\cline { 2 - 6 } & & AMBER & AMBER $(\mathrm{p})$ & MORSE & MORSE $(\mathrm{p})$ \\
\cline { 2 - 6 } & $\mathrm{d}(\mathrm{nm})$ & $\mathrm{E}(\mathrm{GPa})$ & $\mathrm{E}(\mathrm{GPa})$ & $\mathrm{E}(\mathrm{GPa})$ & $\mathrm{E}(\mathrm{GPa})$ \\
\hline $\mathrm{ZZ}(9,0)$ & 0.7046 & 652.25 & 661.96 & 742.78 & 758.32 \\
$\mathrm{ZZ}(11,0)$ & 0.8612 & 707.34 & 714.23 & 807.76 & 818.36 \\
$\mathrm{ZZ}(13,0)$ & 1.0178 & 733.35 & 741.34 & 841.04 & 853.42 \\
\hline $\mathrm{AC}(5,5)$ & 0.6780 & 655.01 & 652.39 & 716.82 & 749.63 \\
$\mathrm{AC}(7,7)$ & 0.9492 & 714.88 & 710.40 & 793.20 & 816.92 \\
$\mathrm{AC}(8,8)$ & 1.0848 & 709.03 & 727.62 & 811.29 & 837.80 \\
\hline $\mathrm{CH}(7,4)$ & 0.7541 & 758.04 & 789.15 & 893.94 & 689.50 \\
$\mathrm{CH}(8,5)$ & 0.8879 & 614.42 & 721.82 & 782.10 & 839.13 \\
$\mathrm{CH}(10,6)$ & 1.0960 & 607.76 & 710.09 & 781.07 & 821.92 \\
\hline
\end{tabular}

Tabla 4.12: Módulo de Young equivalente a flexión 
Por otra parte, los SWNTs son mucho más deformables transversalmente que en la dirección longitudinal [38], [81], [92] (anisotropía global), lo cual refuerza la no aplicabilidad del continuo para su análisis mecánico empleando unas constantes elásticas únicas.

Aunque no hay una diferencia clara entre los módulos de Young de nanotubos ZZ y AC, no se puede afirmar que la respuesta a flexión sea independiente de la quiralidad, ya que los valores de $E$ son muy diferentes respecto a los casos aquirales para diámetros sensiblemente iguales. Se puede deducir entonces que la falta de axisimetría tiene una influencia notable en la rigidez a flexión.

Representamos en la figura 4.21 los valores de la tabla 4.12. Como se puede ver, el módulo de Young es creciente con el diámetro para nanotubos ZZ y AC, pero sorprendentemente es decreciente para nanotubos $\mathrm{CH}$. Conviene señalar que el mal condicionamiento numérico expuesto en el comentario 4.3.1 es particularmente importante en SWNTs Chiral de pequeño diámetro a flexión, constituyendo la causa más probable de esta tendencia contradictoria.

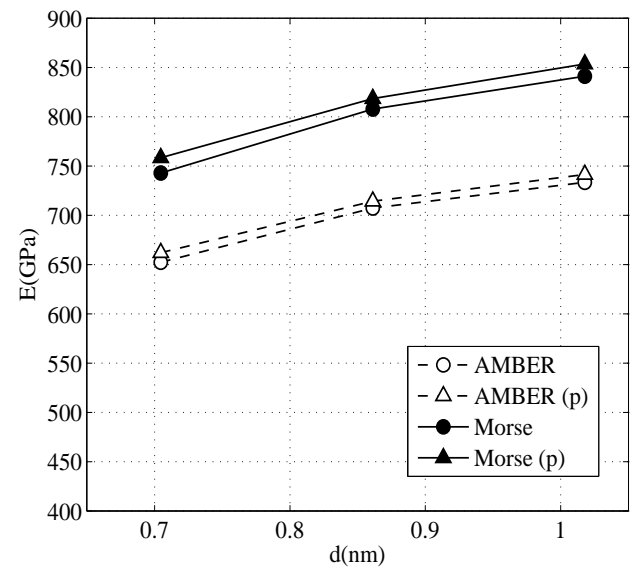

(a) SWNTs ZigZag flexión

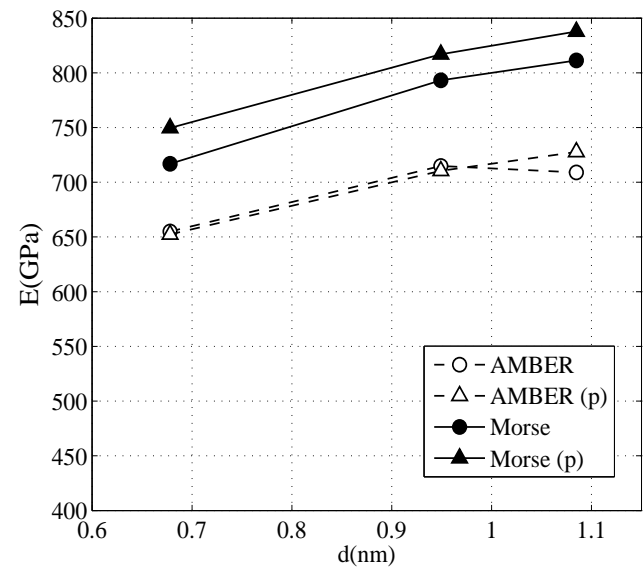

(b) SWNTs Armchair flexión

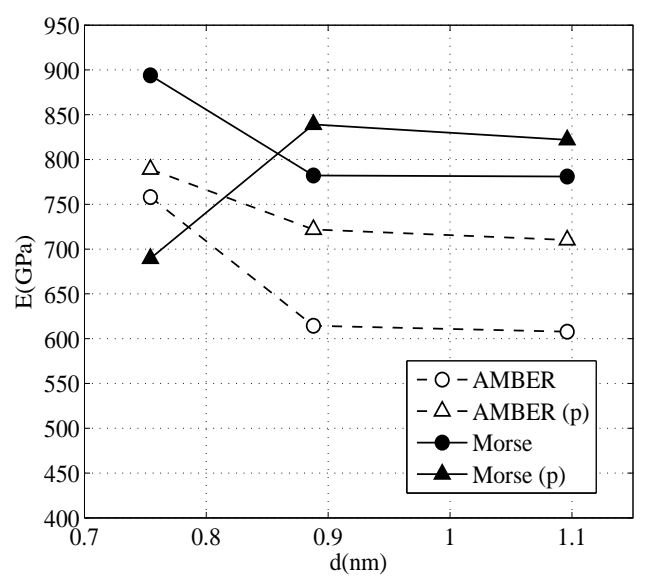

(c) SWNTs Chiral flexión

Figura 4.21: Variación del Módulo de Young a flexión con el diámetro

Finalmente, representamos algunas geometrías deformadas de SWNTs a flexión en la figura 4.22 


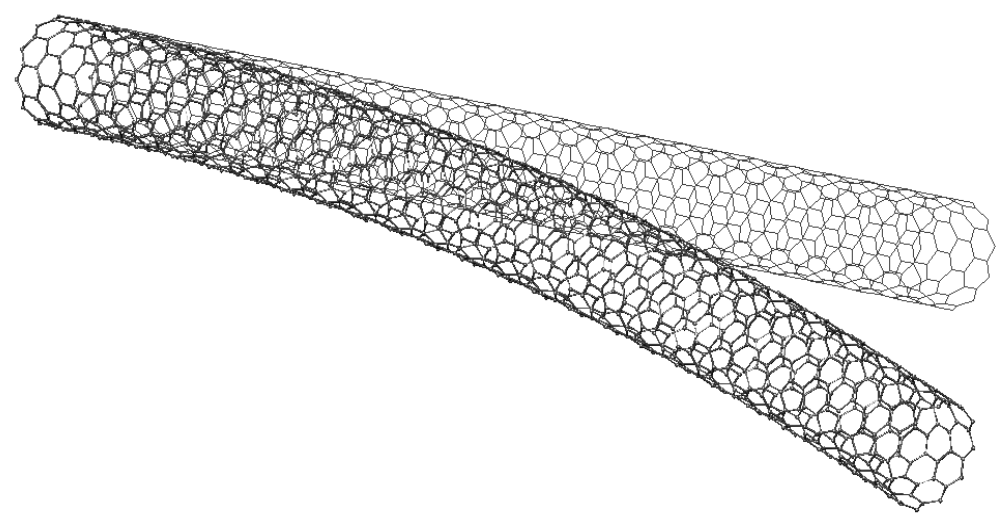

(a) $\mathrm{ZZ}(11,0) M=2 \mathrm{nN} \cdot \mathrm{nm}, \mathrm{AMBER}(\mathrm{p})$

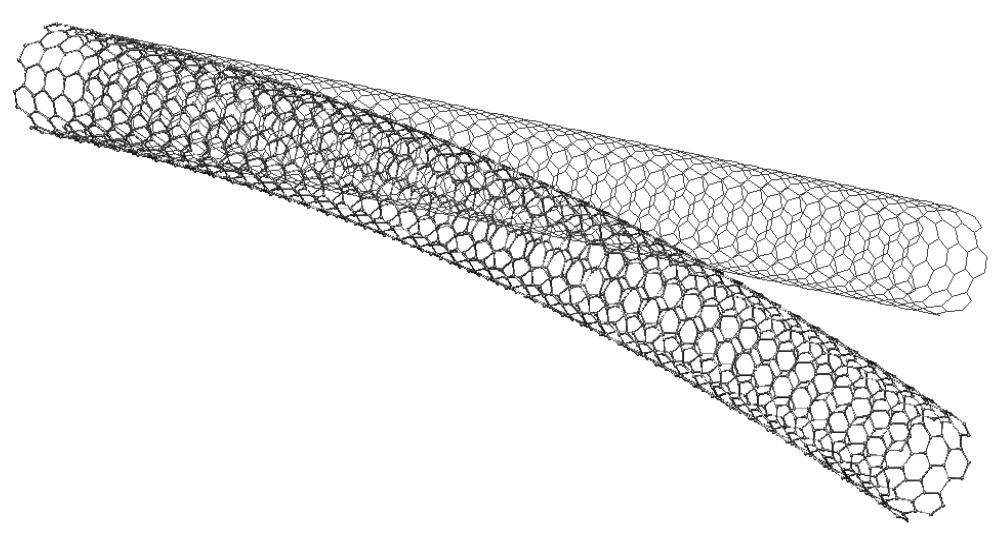

(b) $\operatorname{AC}(7,7) M=3 \mathrm{nN} \cdot \mathrm{nm}, \operatorname{MORSE}(\mathrm{p})$

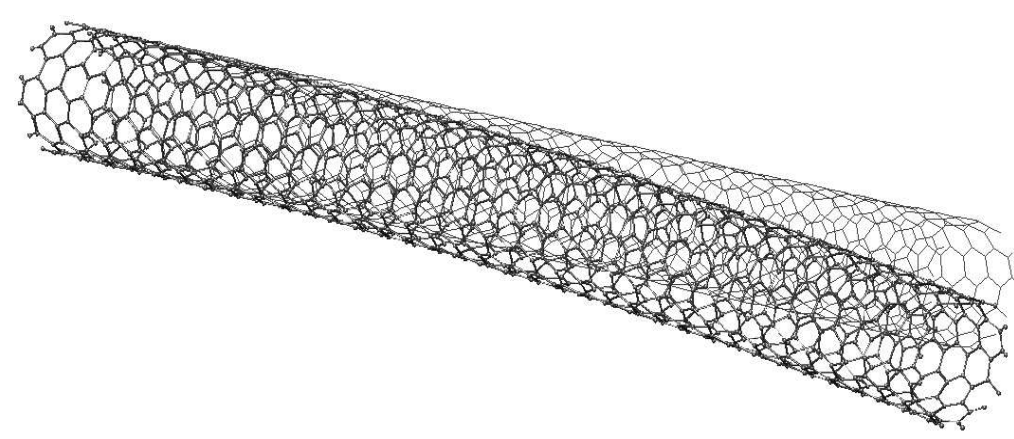

(c) $\mathrm{CH}(8,5) M=1 \mathrm{nN} \cdot \mathrm{nm}, \operatorname{MORSE}(\mathrm{p})$

Figura 4.22: Algunas geometrías deformadas de SWNTs a flexión 


\subsubsection{Comportamiento a torsión}

Para completar los esquemas de carga habituales que pueden actuar sobre un elemento estructural tipo pieza alargada, sometemos los SWNTs en ménsula (vinculados mediante una corona de apoyos articulados) a un conjunto de cargas puntuales $F_{t i}$ en el extremo libre que producirán un par torsor con los mismos valores adoptados en flexión (ver tabla 4.10). Estas cargas se introducen en la dirección tangente a la circunferencia que circunscribe la sección transversal del extremo libre y son estáticamente equivalentes al torsor aplicado, luego su módulo es

$$
F_{t i}=\frac{2 M_{t}}{N_{t} d}
$$

y sus componentes se obtienen proyectándolas sobre los ejes globales. Los puntos de aplicación y los valores numéricos de las componentes finales de estas fuerzas para un torsor de $M_{t}=1 \mathrm{nN} \cdot \mathrm{nm}$ se proporcionan en la tabla 4.13

\begin{tabular}{ccccccccccccc}
\hline \multicolumn{4}{c}{$\mathrm{ZZ}(11,0)$} & \multicolumn{1}{c}{$\mathrm{AC}(5,5)$} & \multicolumn{4}{c}{$\mathrm{CH}(7,4)$} \\
\hline$y_{i}(\mathrm{~nm})$ & $z_{i}(\mathrm{~nm})$ & $R_{y i}(\mathrm{nN})$ & $R_{z i}(\mathrm{nN})$ & $y_{i}(\mathrm{~nm})$ & $z_{i}(\mathrm{~nm})$ & $R_{y i}(\mathrm{nN})$ & $R_{z i}(\mathrm{nN})$ & $y_{i}(\mathrm{~nm})$ & $z_{i}(\mathrm{~nm})$ & $R_{y i}(\mathrm{nN}) R_{z i}(\mathrm{nN})$ \\
\hline 0.0613 & -0.4262 & 0.21 & 0.03 & 0.1048 & -0.3224 & 0.28 & 0.09 & 0.0394 & -0.3754 & 0.24 & 0.03 \\
0.2820 & -0.3254 & 0.16 & 0.14 & 0.2268 & -0.2520 & 0.22 & 0.20 & 0.1888 & -0.3269 & 0.21 & 0.12 \\
0.4131 & -0.1213 & 0.06 & 0.20 & 0.3390 & 0.0000 & 0.00 & 0.29 & 0.3547 & -0.1291 & 0.08 & 0.23 \\
0.4131 & 0.1213 & -0.06 & 0.20 & 0.3097 & 0.1379 & -0.12 & 0.27 & 0.3693 & 0.0784 & -0.05 & 0.24 \\
0.2820 & 0.3254 & -0.16 & 0.14 & 0.1048 & 0.3224 & -0.28 & 0.09 & 0.2892 & 0.2427 & -0.15 & 0.18 \\
0.0613 & 0.4262 & -0.21 & 0.03 & -0.0354 & 0.3371 & -0.27 & -0.03 & 0.0655 & 0.3718 & -0.24 & 0.04 \\
-0.1789 & 0.3917 & -0.19 & -0.09 & -0.2743 & 0.1993 & -0.17 & -0.24 & -0.1166 & 0.3590 & -0.23 & -0.07 \\
-0.3622 & 0.0233 & -0.11 & -0.18 & -0.3316 & 0.0705 & -0.06 & -0.29 & -0.2892 & 0.2426 & -0.15 & -0.18 \\
-0.4306 & 0.0000 & -0.21 & 0.00 & -0.2743 & -0.1993 & 0.17 & -0.24 & -0.3775 & 0.0000 & 0.00 & -0.24 \\
-0.3622 & -0.2328 & 0.11 & -0.18 & -0.1695 & -0.2936 & 0.26 & -0.15 & -0.3449 & -0.1535 & 0.10 & -0.22 \\
-0.1789 & -0.3917 & 0.19 & -0.09 & & & & & -0.1887 & -0.3269 & 0.21 & -0.12 \\
\hline
\end{tabular}

Tabla 4.13: Cargas aplicadas a torsión para $M_{t}=1 \mathrm{nN} \cdot \mathrm{nm}$

Representamos en la figura 4.23 las curvas momento-rotación en el extremo libre, que reproducen la respuesta a torsión de SWNTs, comparadas con las curvas que se obtendrían a partir del módulo de deformación transversal $G$ obtenido en [15] aunque empleando las rigideces AMBER. Para una mayor brevedad, se incluye sólo la respuesta de nanotubos ZZ y AC. En este caso no comparamos nuestros resultados con [78] porque la citada referencia obtiene valores de $G$ sin considerar la curvatura del nanotubo.

El momento aplicado $M_{t}$ y la rotación en el extremo libre $\theta$ se suponen sujetos a la relación de torsión de Coulomb:

$$
M_{t}=\frac{G I_{0}}{L} \theta
$$

donde $I_{0}$ representa el momento de inercia polar de la sección transversal considerada como un anillo continuo de espesor $t=0.34 \mathrm{~nm}$. A partir de (4.36), podemos deducir un módulo de deformación transversal equivalente como:

$$
G=\frac{M_{t} L}{I_{0} \theta}
$$

Promediando valores para cada nanotubo, obtenemos los valores definitivos de la tabla 4.14 Teniendo en cuenta que los valores de [15] oscilan entre 308.5 - 312.5 GPa para SWNTs ZigZag y entre 292 - 306.5 GPa para SWNTs Armchair, con la presente formulación obtenemos unos resultados hasta un $24 \%$ más flexibles. A pesar de ello, la concordancia en términos de orden de magnitud resulta aceptable. 


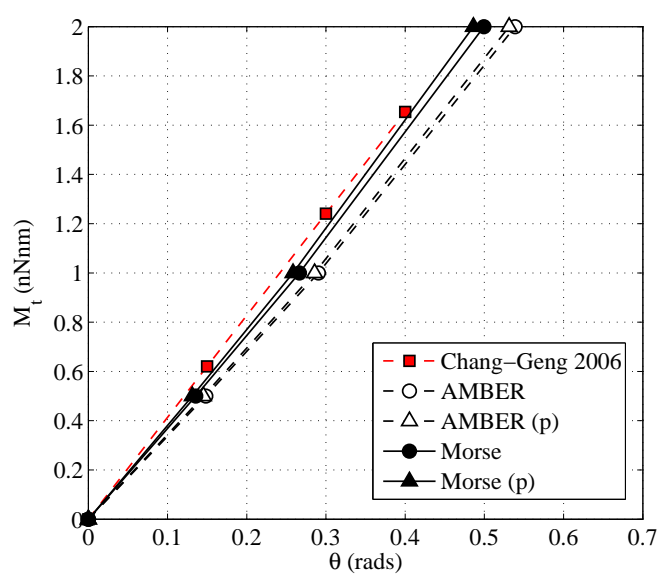

(a) $\mathrm{ZZ}(9,0)$ torsión

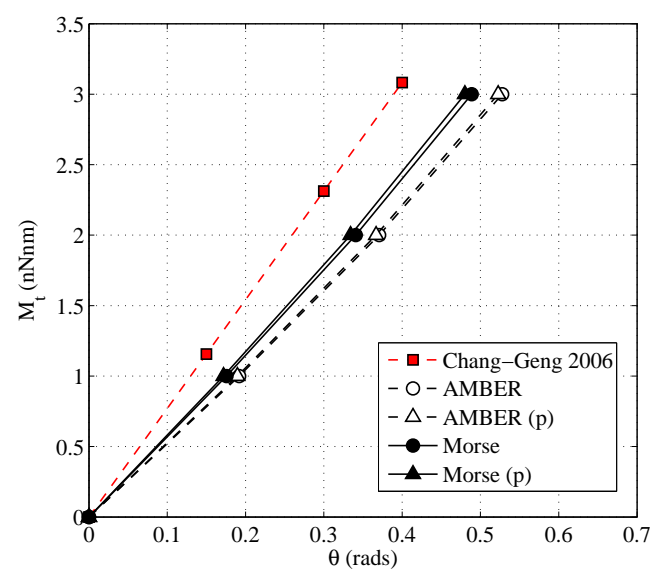

(c) ZZ $(11,0)$ torsión

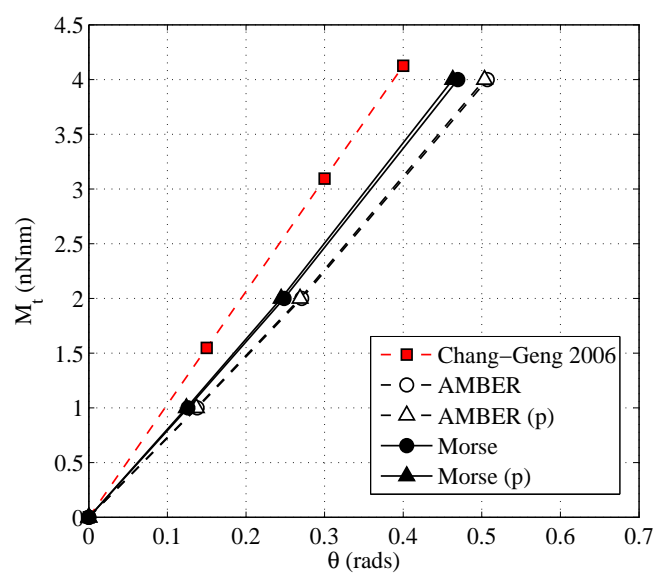

(e) $\mathrm{ZZ}(13,0)$ torsión

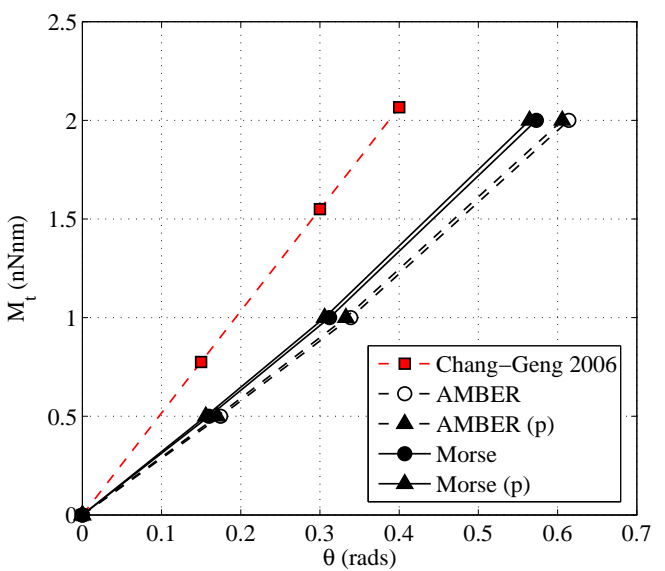

(b) $\mathrm{AC}(5,5)$ torsión

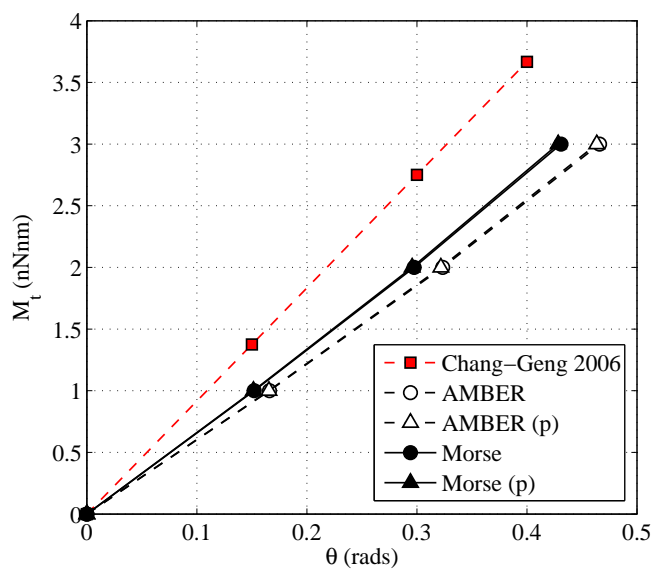

(d) $\mathrm{AC}(7,7)$ torsión

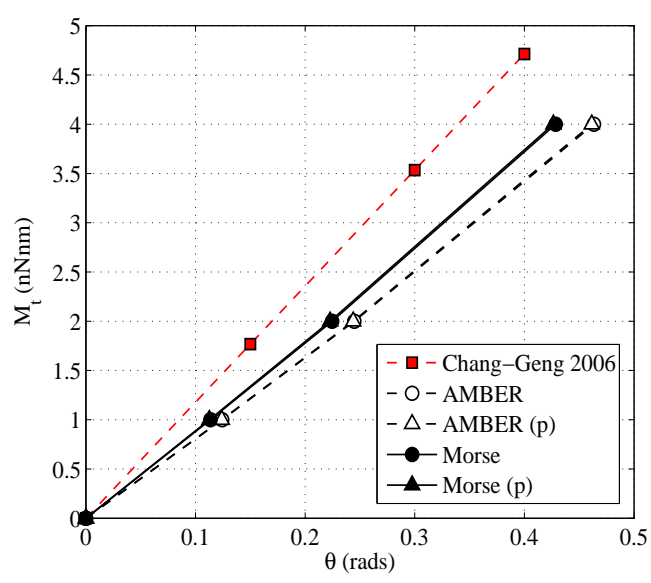

(f) $\mathrm{AC}(8,8)$ torsión

Figura 4.23: SWNTs Armchair y ZigZag a torsión, comparación con [15] 
Capítulo 4. Formulación general del modelo y su aplicación a nanotubos monocapa

\begin{tabular}{lccccc} 
& & AMBER & AMBER $(\mathrm{p})$ & MORSE & MORSE $(\mathrm{p})$ \\
\cline { 2 - 6 } & $\mathrm{d}(\mathrm{nm})$ & $\mathrm{G}(\mathrm{GPa})$ & $\mathrm{G}(\mathrm{GPa})$ & $\mathrm{G}(\mathrm{GPa})$ & $\mathrm{G}(\mathrm{GPa})$ \\
\hline $\mathrm{ZZ}(9,0)$ & 0.7046 & 261.74 & 266.22 & 284.62 & 293.15 \\
$\mathrm{ZZ}(11,0)$ & 0.8612 & 283.62 & 286.64 & 308.14 & 314.31 \\
$\mathrm{ZZ}(13,0)$ & 1.0178 & 292.91 & 295.15 & 318.70 & 323.42 \\
\hline $\mathrm{AC}(5,5)$ & 0.6780 & 235.67 & 240.04 & 255.05 & 260.29 \\
$\mathrm{AC}(7,7)$ & 0.9492 & 272.82 & 274.13 & 296.49 & 298.09 \\
$\mathrm{AC}(8,8)$ & 1.0848 & 282.88 & 283.91 & 307.83 & 310.15 \\
\hline $\mathrm{CH}(7,4)$ & 0.7541 & 413.04 & 224.00 & 185.06 & 304.67 \\
$\mathrm{CH}(8,5)$ & 0.8879 & 248.60 & 218.73 & 291.59 & 228.65 \\
$\mathrm{CH}(10,6)$ & 1.0960 & 269.80 & 260.86 & 306.30 & 251.50 \\
\hline
\end{tabular}

Tabla 4.14: Módulo de deformación transversal de equivalente a torsión

La variación del módulo de deformación transversal respecto al diámetro del nanotubo se reproduce en la figura 4.24. Como puede verificarse, nuestros resultados muestran la misma tendencia creciente con el diámetro obtenida en [15, fig 4], [61, fig 7] y [114, fig 10], aunque con unos valores inferiores (alrededor del 20\%). Además, el potencial Morse parece producir valores más rígidos de $G$ que el AMBER, aunque esta diferencia no es importante en nuestro rango de diámetros.

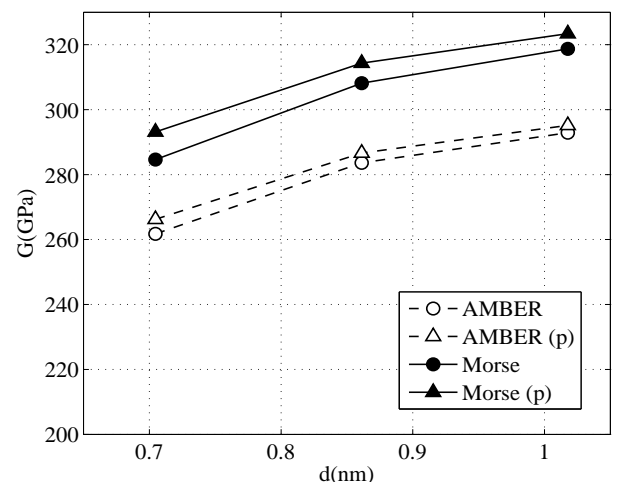

(a) SWNTs ZigZag a torsión

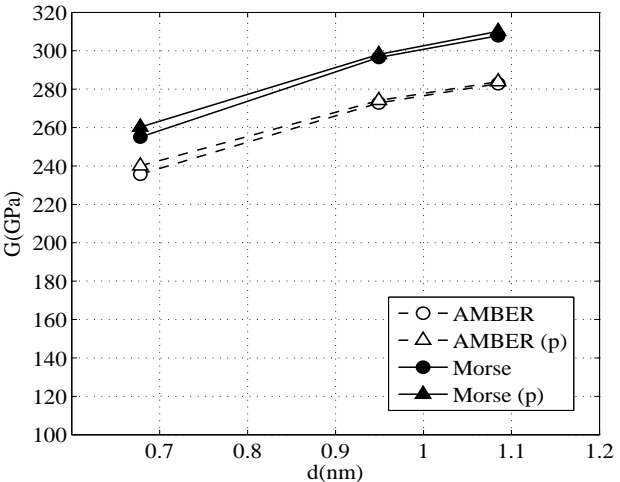

(b) SWNTs Armchair a torsión

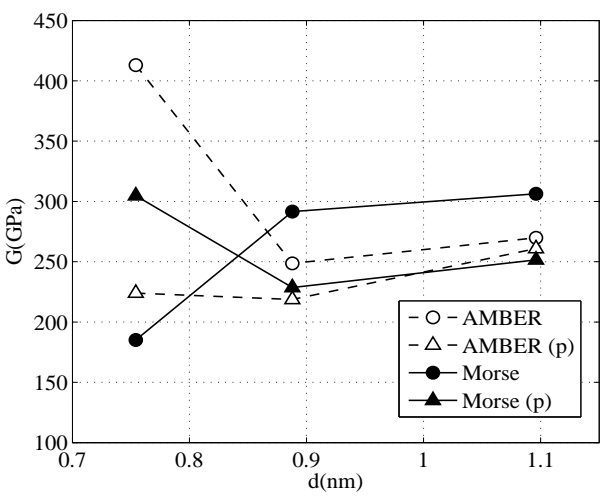

(c) SWNTs Chiral a torsión

Figura 4.24: Variación del módulo de deformación transversal con el diámetro 
Como puede verse en la figura 4.24(c), el mal condicionamiento de los nanotubos $\mathrm{CH}$ con potencial Morse también está presente a torsión. Asimismo, la falta de axisimetría de estos nanotubos lleva a excentricidades en el sistema de cargas aplicadas en el extremo libre que provoca cierta flexión; este acoplamiento flexión-torsión desvirtúa los resultados finales (figura 4.27). Como hemos mencionado anteriormente, este problema se puede resolver mediante un sistema de desplazamientos impuestos en lugar de cargas puntuales en el extremo libre.

$\mathrm{Si}$ observamos con detalle las figuras $4.25 \mathrm{a} 4.27$, se puede comprender mejor el comportamiento a torsión de SWNTs. Como se puede comprobar, al considerar las tensiones iniciales en el análisis, el acortamiento habitual de SWNTs vuelve a aparecer a torsión. Sin embargo, en este caso no procede modificar el origen de deformaciones (Comentario 4.3.2) al incluir el pretensado, para medir adecuadamente las rotaciones longitudinales del extremo libre.

Por otra parte, conforme aumenta el momento torsor aparece una deformación radial de la sección transversal en el extremo libre con ambas funciones potenciales (fig. 4.26). Puesto que las cargas puntuales se aplican en la dirección tangente a la circunferencia extrema, tienden a desplazar los átomos del extremo libre hacia el exterior de tal circunferencia. En consecuencia, este primer conjunto de átomos arrastran a sus vecinos y se provoca una deformación cónica. Este modo de deformación no deseado es la causa más probable de la respuesta $M-\theta$ no estrictamente lineal (ver fig. 4.23), incluso con potencial AMBER.

Pese a que los nanotubos $\mathrm{ZZ}$ parecen algo más rígidos a torsión que los $\mathrm{AC}$, las diferencias no son determinantes. No obstante, los valores de $G$ difieren bastante de los asociados a nanotubos aquirales (como ocurría en flexión), por tanto no se puede afirmar que la respuesta a torsión sea independiente de la quiralidad.

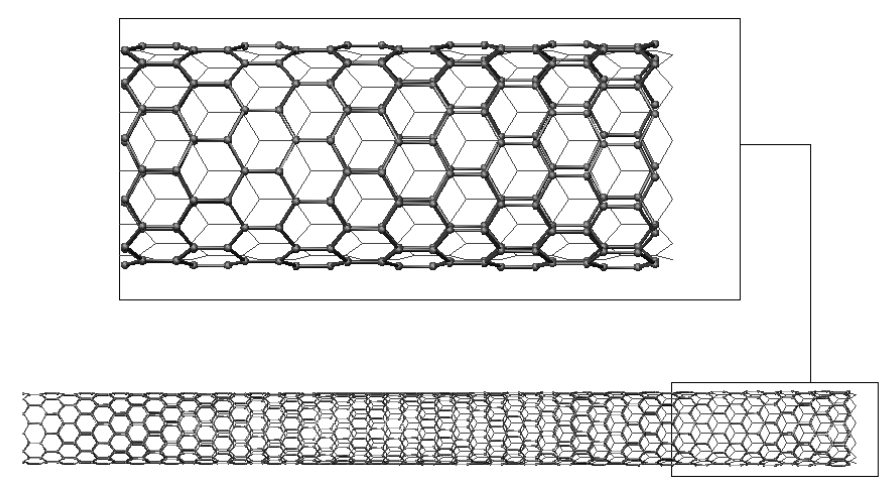

Figura 4.25: $Z Z(11,0) M=2 n N \cdot n m, M O R S E(p)$ 


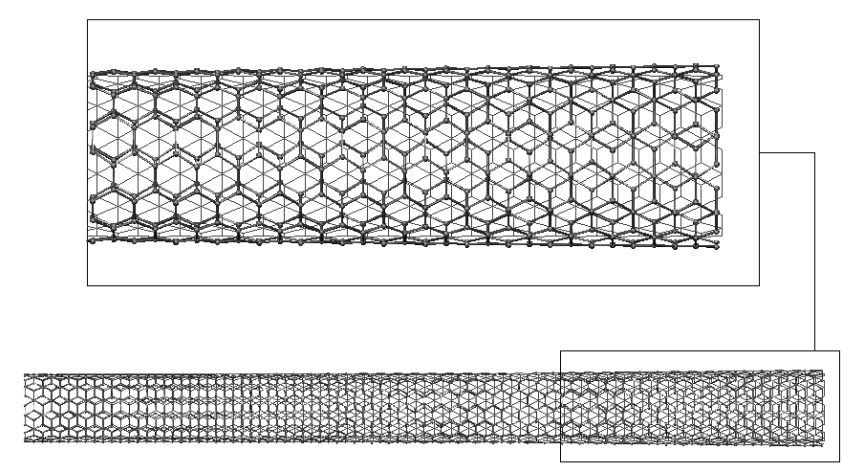

Figura 4.26: $A C(7,7) M=3 n N \cdot n m, A M B E R(p)$

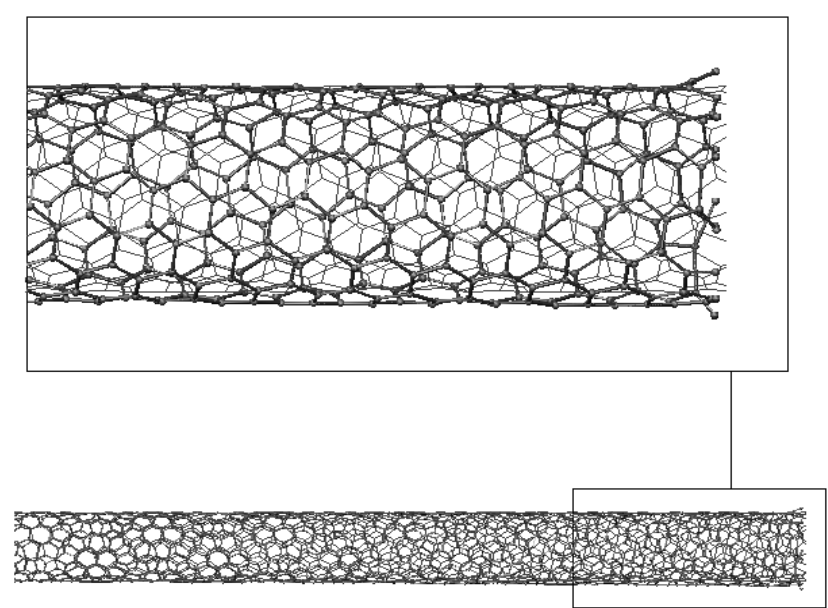

Figura 4.27: $C H(8,5) M=2 n N \cdot n m, A M B E R(p)$

\subsubsection{Deducción del coeficiente de Poisson}

Como se ha mencionado previamente en este capítulo, los modelos continuos no son aplicables a la nanoescala de los SWNTs. Con el propósito de subrayar esta idea, se obtuvieron valores del coeficiente de Poisson a través de la relación constitutiva clásica:

$$
v=\frac{E}{2 G}-1
$$

Análogamente a lo expuesto en [15], si sustituimos en la relación anterior el módulo de Young obtenido a tracción y el módulo de deformación transversal obtenido a torsión, resultan los valores resumidos en la tabla 4.15 Igualmente, la variación del coeficiente de Poisson con respecto al diámetro se representa en la figura 4.28

Partiendo de la figura 4.28(c), se puede afirmar que los resultados para SWNTs Chiral no son fiables debido a los mismos problemas de mal condicionamiento numérico encontrado a torsión con potencial Morse. Evidentemente, los coeficientes de Poisson negativos carecen de cualquier sentido físico. Por otra parte, si prestamos atención a los resultados para SWNTs ZigZag y Armchair deducimos una tendencia decreciente del coeficiente de Poisson con el diámetro hasta un valor medio de 0.25-0.3. Sin embargo, la extraordinaria dispersión en los valores finales hace que esta tendencia no sea completamente fiable. 


\begin{tabular}{lcccc} 
& AMBER & AMBER $(\mathrm{p})$ & MORSE & MORSE $(\mathrm{p})$ \\
\cline { 2 - 5 } & $v$ & $v$ & $v$ & $v$ \\
\hline $\mathrm{ZZ}(9,0)$ & 0.48 & 0.45 & 0.58 & 0.53 \\
$\mathrm{ZZ}(11,0)$ & 0.38 & 0.37 & 0.48 & 0.45 \\
$\mathrm{ZZ}(13,0)$ & 0.35 & 0.34 & 0.44 & 0.42 \\
\hline $\mathrm{AC}(5,5)$ & 0.67 & 0.64 & 0.79 & 0.76 \\
$\mathrm{AC}(7,7)$ & 0.46 & 0.45 & 0.56 & 0.55 \\
$\mathrm{AC}(8,8)$ & 0.41 & 0.40 & 0.51 & 0.50 \\
\hline $\mathrm{CH}(7,4)$ & -0.06 & 0.71 & 1.45 & 0.49 \\
$\mathrm{CH}(8,5)$ & 0.58 & 0.77 & 0.54 & 1.00 \\
$\mathrm{CH}(10,6)$ & 0.45 & 0.49 & 0.48 & 0.84 \\
\hline
\end{tabular}

Tabla 4.15: Coeficiente de Poisson a partir de tracción y torsión

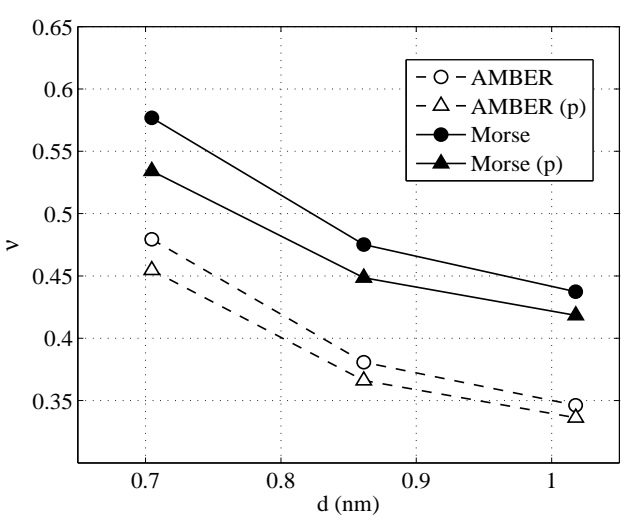

(a) ZigZag CNTs

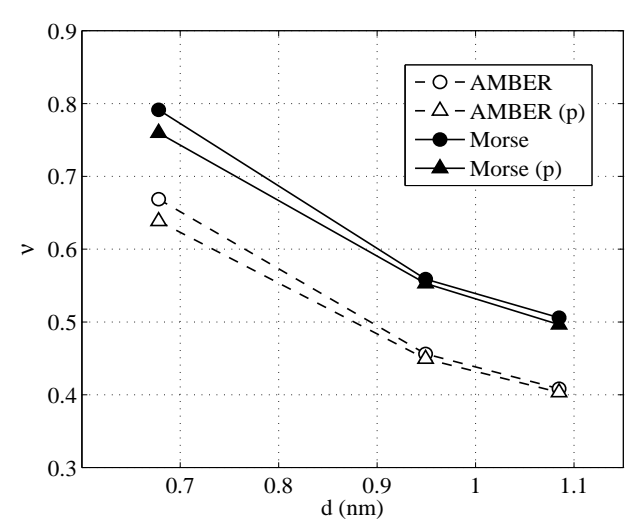

(b) Armchair CNTs

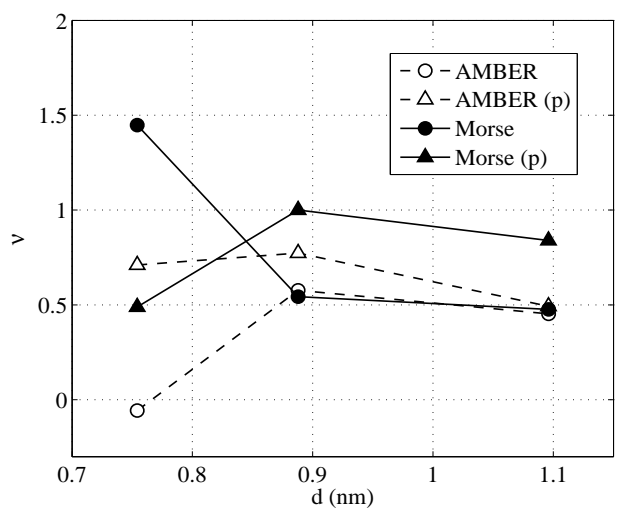

(c) Chiral CNTs

Figura 4.28: Variación del coeficiente de Poisson con el diámetro

En resumen, los parámetros mecánicos equivalentes obtenidos a partir del modelo atómico MSM no verifican la ecuación constitutiva 4.38 propia de un medio continuo. Este hecho pone de manifiesto que el comportamiento mecánico de los SWNTs no se puede reproducir correctamente mediante modelos viga (o lámina) basados en los parámetros mecánicos de la teoría de la elasticidad, 
de acuerdo con [15]. En una línea algo diferente, [78] proponen un valor de $v=0.273$ y sugieren que dicha ecuación se puede emplear para estimar el módulo de Young de una lámina de grafeno plana, pero su validez no se puede extender a los SWNTs.

\subsection{Sumario del análisis lineal}

Resumimos a continuación las ideas fundamentales extraídas del análisis geométricamente lineal de SWNTs mediante la presente formulación general en rigidez del modelo MSM:

1. La gran versatilidad de la presente formulación general en rigidez del modelo stick-spiral ha sido puesta de manifiesto mediante la simulación de SWNTs frente a cuatro estados de carga generales (tracción, compresión, flexión y torsión). Esta característica representa una importante novedad frente a la mayoría de referencias consultadas relacionadas con el mismo modelo. Incluso en [15], donde se incluye el tratamiento de nanotubos Chiral, sólo se trata la tracción, la presión interior al cilindro y la torsión mediante un complejo desarrollo trigonométrico que no deja de ser una formulación parcial frente a la presentada en esta tesis.

2. El potencial de Morse proporciona resultados más rígidos a tracción que el AMBER con diferencias en torno al $15 \%$. Idéntico efecto rigidizador producido por la función de Morse (15\% a compresión, hasta un $13 \%$ a flexión y cerca de un $8 \%$ a torsión) está presente en todos los casos de carga estudiados. Sin embargo, no aparecen diferencias importantes para deformaciones por debajo del $6 \%$ a tracción y del $4 \%$ a compresión.

3. A pesar de la naturaleza no lineal del potencial Morse, las simulaciones de SWNTs que lo emplean producen una respuesta lineal bajo cualquier caso de carga. Esto es debido a que las rigideces tangentes apenas varían en el rango de cargas considerado. Además, debe notarse que los mayores niveles de deformación estudiados no son consistentes con la formulación geométricamente lineal del modelo, la cual está orientada a plasmar la respuesta mecánica de SWNTs bajo la hipótesis de pequeños desplazamientos. De ahí que la formulación geométricamente no lineal del modelo (capítulo 5) aparece como una extensión natural del análisis.

4. La formulación general desarrollada en este capítulo permite incluir las tensiones iniciales explícitamente sin más que sumar un sistema de deformaciones iniciales en la ecuación constitutiva (4.5). Esta característica constituye una novedad importante respecto a otros trabajos que versan sobre en modelo stick-spiral [14], [15],[77], [78], [114]. Estas deformaciones iniciales se estiman asumiendo que los átomos de carbono permanecen en la superficie cilíndrica en su geometría no deformada (mapeo conforme), y considerando que los enlaces covalentes están contenidos en las secantes que unen dos átomos de $\mathrm{C}$ adyacentes.

5. El pretensado introduce fuerzas resultantes radiales que tienden a mantener la geometría circular de la sección transversal del nanotubo. Así, el efecto Poisson convierte las fuerzas radiales en acortamiento longitudinal bajo cualquier estado de cargas externas. No obstante, teniendo en cuenta la configuración de apoyos adoptada (tipo ménsula) y la necesaria modificación del origen de deformaciones longitudinales (comentario 4.3.2), no se produce prácticamente ninguna diferencia en la rigidez a tracción y compresión de SWNTs debido a los esfuerzos iniciales. Además, los resultados son muy similares a flexión (diferencias en el rango del $5 \%$ ) y torsión (diferencias alrededor del $3 \%$ ) y podemos considerar los casos de carga estudiados como independientes del pretensado.

6. A pesar de la adopción del módulo de Young y de deformación transversal como parámetros mecánicos para la comparación de resultados, esta elección puede resultar controvertida debido 
a su elevada dependencia del espesor de pared equivalente (adoptado habitualmente como 0.34 $\mathrm{nm})$. En nuestro caso, nuestro único objetivo ha sido validar nuestro modelo a partir de los valores de estos parámetros presentes en la literatura mediante formulaciones parciales del modelo atómico stick-spiral, pero no cuantificar los parámetros mecánicos asociados con el continuo.

7. De hecho, es dudoso tratar los SWNTs como un medio continuo, como se ha demostrado a través de la gran dispersión observada en el coeficiente de Poisson calculado mediante la ecuación (4.38). En definitiva, las relaciones constitutivas clásicas no reproducen adecuadamente el comportamiento de un medio continuo equivalente a los SWNTs.

8. Los SWNTs ZigZag son más rígidos que los Armchair a tracción (hasta un $8 \%$ ), pero las diferencias entre ambos a compresión, flexión y torsión no es determinante. No obstante, no se puede afirmar que la respuesta mecánica sea independiente de la quiralidad a flexión y torsión, ya que los nanotubos Chiral poseen valores de $E$ y $G$ bastante diferentes a los obtenidos para los nanotubos aquirales bajo dichos estados de carga.

9. El módulo de Young $E$ es ligeramente creciente con el diámetro del nanotubo a tracción y compresión, aunque se puede considerar prácticamente constante en la respuesta longitudinal. Además, E es creciente bajo cargas de flexión y el módulo de deformación por cortante G es creciente bajo cargas de torsión con el diámetro del CNT. En ambos casos los resultados de los nanotubos $\mathrm{CH}$ con potencial Morse se ven desvirtuados por el mal condicionamiento numérico experimentado. Esta dificultad puede encontrar su causa en la falta de axisimetría de los nanotubos $\mathrm{CH}$. Al someter estos SWNTs a las acciones externas, algunos enlaces aparecen fuertemente traccionados y otros fuertemente comprimidos. En consecuencia, la extremada diferencia entre las rigideces de enlace a tracción y compresión que proporciona el potencial Morse induce ciertas singularidades numéricas.

10. Como salida de resultados de los códigos propios desarrollados, se obtienen axiles de enlace, momentos angulares y reacciones de apoyos, además de las geometrías deformadas. Mediante la interpretación cualitativa de las deformadas resultantes, se han descubierto algunas deformaciones locales no deseadas en el extremo libre, sobre todo con nanotubos $\mathrm{CH}$. Este efecto se debe probablemente a la configuración local de los "enlaces" en los extremos del nanotubo. En particular, algunos elementos barra en estas zonas son más cortos que los enlaces covalentes $C-C$ reales y la aplicación de un conjunto de fuerzas puntuales desencadenará deformaciones locales inesperadas. En cualquier caso, este problema se puede eliminar introduciendo desplazamientos impuestos en el extremo libre mediante apoyos articulados móviles. No obstante, esta solución puede producir valores ligeramente diferentes de los parámetros mecánicos (como se demuestra en [41] y en contra de [73]). A pesar de ello, las deformaciones locales cerca de los extremos del nanotubo deben considerarse como efectos locales despreciables. 



\section{Capítulo 5}

\section{Extensión de la formulación a la no linealidad geométrica}

Dedicamos el presente capítulo al análisis geométricamente no lineal de SWNTs extendiendo la formulación general del modelo stick-spiral desarrollada en el capítulo4. Teniendo en cuenta las limitaciones asociadas a la hipótesis de pequeños desplazamientos, como por ejemplo la incapacidad de captar deformaciones críticas de inestabilidad, consideraremos que los desplazamientos en los desarrollos que siguen son moderados.

En el campo de las aplicaciones tecnológicas, los CNTs presentan prometedoras aplicaciones en el refuerzo de composites de matriz polimérica, a pesar de las dificultades en la transferencia de carga entre nanotubo y matriz. Partiendo de [82], donde se examina la inestabilidad de nanofibras dentro de una matriz de polímero y se enfatiza que el fallo por pandeo o abolladura puede ser el modo determinante de colapso incluso en composites, se considera fundamental el desarrollo del análisis geométricamente no lineal en el marco del modelo MSM.

Aunque en los polímeros reforzados con CNTs, la resistencia viene condicionada por la interacción entre SWNTs dentro de un fajo o entre capas de un MWNT, este desarrollo (aplicado a SWNTs) puede considerarse como una aproximación inicial al posterior entendimiento del comportamiento no lineal de fajos de SWNTs dentro de la matriz.

Por otra parte, dentro del análisis no lineal cobran especial importancia las ventajas relacionadas con el rendimiento numérico de los modelos MSM frente a las técnicas MD. En efecto, dado el coste computacional empleado en la evaluación de la matriz de rigidez tangente y la resolución del sistema lineal incremental en rigidez, la reducción en términos de grados de libertad del modelo empleado resulta especialmente significativa.

Cabe señalar que la respuesta real de SWNTs aislados frente a fenómenos de inestabilidad está altamente influenciada por la fluctuación térmica aleatoria de cada átomo en torno a su posición teórica de equilibrio. Puesto que en los modelos MM no se considera ninguna transmisión de calor con el entorno, esto presenta una limitación de nuestro modelo frente a las técnicas MD. A pesar de este hecho, el acoplamiento entre la vibración térmica y los defectos estructurales dificulta el análisis de los fenómenos de inestabilidad intrínseca de SWNTs via métodos MD. En este sentido, los modelos MM o MSM constituyen herramientas interesantes como primer paso hacia el análisis del comportamiento frente a la inestabilidad. 


\subsection{Ecuaciones de campo de la formulación no lineal}

Dentro del análisis geométricamente no lineal de SWNTs, separamos el conjunto de apoyos en un grupo de apoyos fijos y otro de apoyos móviles que serán los responsables de introducir las acciones externas. Esta nueva concepción del método de carga persigue evitar las deformaciones locales no deseadas presentes en los bordes del nanotubo (ver capítulo 4). Para ello, se definen las siguientes variables:

$\mathbf{u}_{R} \quad$ desplazamientos de los nudos libres

$\mathbf{u}_{A}$ desplazamientos de los nudos coaccionados (serán nulos)

$\mathbf{u}_{M} \quad$ desplazamientos de los apoyos móviles. Supondremos que $\mathbf{u}_{M}=\overline{\mathbf{u}}$ son funciones conocidas de desplazamiento impuesto

$\mathbf{f}_{R} \quad$ fuerzas externas aplicadas en los nudos libres

$\mathbf{f}_{A} \quad$ reacciones en los apoyos fijos

$\mathbf{f}_{M} \quad$ reacciones en los apoyos móviles

\subsubsection{Ecuaciones cinemáticas}

\section{Elemento barra}

Definimos la siguiente medida no lineal de la deformación longitudinal para el elemento barra $a$ (figura 3.2) entre dos átomos conectados:

$$
e^{a}=\frac{l^{a \prime 2}-l^{a 2}}{2 l^{a}}
$$

En adelante, denotamos con ( )’ las variables medidas en la configuración deformada del elemento.

La definición (5.1) está inspirada en la deformación longitudinal no lineal de Green adoptada habitualmente en mecánica estructural (ver por ej. [22], [23], [29]), aunque en nuestro caso tiene dimensiones de longitud. Normalmente, el trabajo virtual a axil de un elemento barra requiere una integración en la longitud si se emplea la deformación de Green clásica. Sin embargo, en el presente modelo podemos considerar las deformaciones longitudinales $e$ y los esfuerzos axiles $N$ como constantes, expresando el trabajo virtual de las fuerzas internas como $N \delta e$ mediante la introducción de la definición (5.1). De esta forma se evita la integración en la longitud.

Establecemos ahora las relaciones entre las coordenadas y los desplazamientos nodales:

$$
\begin{aligned}
& \mathbf{x}_{i}^{\prime}=\mathbf{x}_{i}+\mathbf{u}_{i}^{a} \\
\mathbf{x}_{j}^{\prime} & =\mathbf{x}_{j}+\mathbf{u}_{j}^{a} \\
\text { y también: }: & \mathbf{x}_{i j}=\mathbf{x}_{j}-\mathbf{x}_{i}=l^{a} \lambda^{a} \\
\mathbf{x}_{i j}^{\prime} & =\mathbf{x}_{j}^{\prime}-\mathbf{x}_{i}^{\prime}=l^{\prime a} \lambda^{\prime a}
\end{aligned}
$$

donde las variables implicadas han sido definidas con anterioridad y pueden consultarse en el Apéndice A Restando (5.2a de 5.2b y empleando (5.2c), 5.2d llegamos a:

$$
\lambda^{\prime a}=\frac{l^{a}}{l^{a}} \lambda^{a}+\frac{\mathbf{u}_{i j}^{a}}{l^{\prime a}} \simeq \lambda^{a}+\frac{\mathbf{u}_{i j}^{a}}{l^{a}}
$$

Se ha adoptado la aproximación $l^{a} \simeq l^{a}$ asumiendo que las deformaciones son pequeñas incluso cuando los desplazamientos son moderados, ya que la no linealidad geométrica es previa a la no

\footnotetext{
${ }^{1}$ Algunas fueron definidas en el capítulo 4 pero se redefinen aquí por claridad en la notación
} 
linealidad constitutiva, como se ha observado experimentalmente por [27] y previsto teóricamente mediante técnicas MD [43], [94], [110].

Llegados a este punto, podemos evaluar el numerador de (5.1) como:

$$
\left(l^{a}\right)^{2}-\left(l^{a}\right)^{2}=\mathbf{x}_{i j}^{\prime T} \mathbf{x}_{i j}^{\prime}-\mathbf{x}_{i j}^{T} \mathbf{x}_{i j}=2\left(\mathbf{x}_{i j}^{a}\right)^{T} \mathbf{u}_{i j}^{a}+\left(\mathbf{u}_{i j}^{a}\right)^{T} \mathbf{u}_{i j}^{a}
$$

alcanzando la siguiente expresión en términos de los desplazamientos nodales para la deformación longitudinal no lineal:

$$
e^{a}=\left(\lambda^{a}\right)^{T} \mathbf{u}_{i j}^{a}+\frac{1}{2 l^{a}}\left(\mathbf{u}_{i j}^{a}\right)^{T} \mathbf{u}_{i j}^{a}
$$

Agrupando en bloques en la ec. (5.5):

$$
\begin{aligned}
e^{a}=\left[\begin{array}{ll}
-\lambda^{a T} & \lambda^{a T}
\end{array}\right]\left\{\begin{array}{l}
\mathbf{u}_{i}^{a} \\
\mathbf{u}_{j}^{a}
\end{array}\right\} & +\frac{1}{2}\left[\begin{array}{ll}
\mathbf{u}_{i}^{a T} & \mathbf{u}_{j}^{a T}
\end{array}\right]\left[\begin{array}{cc}
\mathbf{I} / l^{a} & -\mathbf{I} / l^{a} \\
-\mathbf{I} / l^{a} & \mathbf{I} / l^{a}
\end{array}\right]\left\{\begin{array}{l}
\mathbf{u}_{i}^{a} \\
\mathbf{u}_{j}^{a}
\end{array}\right\}= \\
& =\mathbf{C}_{l}^{a} \mathbf{u}^{a}+\frac{1}{2} \mathbf{u}^{a T} \mathbf{Z}^{a} \mathbf{u}^{a}=\left[\mathbf{C}_{l}^{a}+\frac{1}{2} \mathbf{C}_{n}^{a}\left(\mathbf{u}^{a}\right)\right] \mathbf{u}^{a}=\mathbf{C}^{a}\left(\mathbf{u}^{a}\right) \mathbf{u}^{a}
\end{aligned}
$$

donde:

I matriz identidad de dimensiones $3 \times 3$.

$\mathbf{Z}^{a} \quad$ matriz auxiliar (constante y simétrica).

$\mathbf{C}_{l}^{a} \quad$ matriz cinemática lineal del elemento barra.

$\mathbf{C}_{n}^{a}\left(\mathbf{u}^{a}\right)$ matriz cinemática no lineal del elemento barra.

$\mathbf{C}^{a}\left(\mathbf{u}^{a}\right)$ matriz cinemática total del elemento barra.

Hemos llegado entonces a:

$$
e^{a}=\epsilon^{a}+\frac{1}{2} \mathbf{u}^{a T} \mathbf{Z}^{a} \mathbf{u}^{a}
$$

Por tanto, se puede afirmar que la deformación longitudinal no lineal se descompone en su correspondiente parte lineal y un término cuadrático en los desplazamientos nodales.

\section{Elemento muelle}

La distorsión angular no lineal en el elemento muelle (figura 3.3) se define como la diferencia angular entre dos enlaces covalentes adyacentes (igual que la definición lineal (3.16)) según:

$$
e^{1}=\Delta \gamma^{a b}=A-\alpha
$$

Despejando $\alpha$ en (5.8), tomando cosenos y suponiendo que los desplazamientos son moderados, podemos linealizar la ecuación resultante como sigue:

$$
e^{1}=\frac{\cos \alpha-\cos A}{\sin A}=\frac{\left[\left(\lambda^{a^{\prime}}\right)^{T} \lambda^{b^{\prime}}\right]-\left[\left(\lambda^{a}\right)^{T} \lambda^{b}\right]}{\sin A}
$$

El $\cos \alpha$ en (5.9) se puede evaluar empleando (5.3), luego:

$$
\cos \alpha=\frac{l^{a} l^{b}}{l^{a} l^{b}}\left[\lambda^{a}+\frac{\mathbf{u}_{i j}^{a}}{l^{a}}\right]^{T}\left[\lambda^{b}+\frac{\mathbf{u}_{i k}^{b}}{l^{b}}\right]=\frac{1}{\rho_{l}\left(\mathbf{u}^{a}, \mathbf{u}^{b}\right)}\left[\left(\lambda^{a}\right)^{T} \lambda^{b}+\left(\lambda^{a}\right)^{T} \frac{\mathbf{u}_{i k}^{b}}{l^{b}}+\frac{\left(\mathbf{u}_{i j}^{a}\right)^{T}}{l^{a}} \lambda^{b}+\frac{\left(\mathbf{u}_{i j}^{a}\right)^{T} \mathbf{u}_{i k}^{b}}{l^{a} l^{b}}\right]
$$

donde hemos definido $\rho_{l}\left(\mathbf{u}^{a}, \mathbf{u}^{b}\right)$ como una función de los desplazamientos nodales que puede computarse como:

$$
\rho_{l}\left(\mathbf{u}^{a}, \mathbf{u}^{b}\right)=\frac{l^{a} l^{b}}{l^{a} l^{b}}=\frac{\left(l^{a}+e^{a}\right)\left(l^{b}+e^{b}\right)}{l^{a} l^{b}} \simeq 1+\frac{e^{a}}{l^{a}}+\frac{e^{b}}{l^{b}}=1+\eta\left(\mathbf{u}^{a}, \mathbf{u}^{b}\right)
$$


Nótese que los términos de segundo orden en las deformaciones longitudinales se han despreciado. A continuación, sustituimos la ecuación (5.5) en la función auxiliar $\eta\left(\mathbf{u}^{a}, \mathbf{u}^{b}\right)$ para producir:

$$
\eta\left(\mathbf{u}^{a}, \mathbf{u}^{b}\right)=\frac{\lambda^{a T}}{l^{a}} \mathbf{u}_{i j}^{a}+\frac{1}{2 l^{a}} \mathbf{u}_{i j}^{a T} \mathbf{u}_{i j}^{a}+\frac{\lambda^{b^{T}}}{l^{b}} \mathbf{u}_{i k}^{b}+\frac{1}{2 l^{b^{2}}} \mathbf{u}_{i k}^{b^{T}} \mathbf{u}_{i k}^{b}
$$

Sustituyendo las ecuaciones (5.10) a (5.12) en la definición (5.9) y después de algunas manipulaciones algebraicas, se obtiene:

$$
e^{1}=\left[\frac{\lambda^{b^{T}}}{l^{a} \sin A}-\frac{\lambda^{a T}}{l^{a} \tan A}\right] \mathbf{u}_{i j}^{a}+\left[\frac{\lambda^{a T}}{l^{b} \sin A}-\frac{\lambda^{b^{T}}}{l^{b} \tan A}\right] \mathbf{u}_{i k}^{b}+\frac{\mathbf{u}_{i j}^{a T} \mathbf{u}_{i k}^{b}}{l^{a} l^{b} \sin A}-\frac{\mathbf{u}_{i j}^{a T} \mathbf{u}_{i j}^{a}}{2 l^{a} \tan A}-\frac{\mathbf{u}_{i k}^{b^{T}} \mathbf{u}_{i k}^{b}}{2 l^{b^{2}} \tan A}
$$

Por simplicidad, adoptamos las siguientes definiciones:

$$
\omega_{a}=\frac{1}{l^{a^{2} \tan A}} \quad \omega_{b}=\frac{1}{l^{b^{2} \tan A}} \quad \omega_{a b}=\frac{1}{l^{a} l^{b} \sin A}
$$

empleando (5.14) y las definiciones (3.25) de las funciones $\boldsymbol{\Gamma}$, la ecuación (5.13) se convierte en:

$$
e^{1}=\Gamma_{j}^{1^{T}} \mathbf{u}_{i j}^{a}+\Gamma_{k}^{1^{T}} \mathbf{u}_{i k}^{b}+\omega_{a b} \mathbf{u}_{i j}^{a T} \mathbf{u}_{i k}^{b}-\frac{\omega_{a}}{2} \mathbf{u}_{i j}^{a T} \mathbf{u}_{i j}^{a}-\frac{\omega_{b}}{2} \mathbf{u}_{i k}^{b^{T}} \mathbf{u}_{i k}^{b}
$$

Reordenando términos y agrupando en bloques, 5.14 se puede transformar en:

$$
\begin{aligned}
e^{1}= & {\left[\begin{array}{lll}
\boldsymbol{\Gamma}_{j}^{1^{T}} & \boldsymbol{\Gamma}_{i}^{1^{T}} & \boldsymbol{\Gamma}_{k}^{1^{T}}
\end{array}\right]\left\{\begin{array}{l}
\mathbf{u}_{j}^{1} \\
\mathbf{u}_{i}^{1} \\
\mathbf{u}_{k}^{1}
\end{array}\right\}+} \\
& +\frac{1}{2}\left[\begin{array}{lll}
\mathbf{u}_{j}^{1} & \mathbf{u}_{i}^{1{ }^{T}} & \mathbf{u}_{k}^{1^{T}}
\end{array}\right]\left[\begin{array}{ccc}
-\omega_{a} \mathbf{I} & \left(\omega_{a}-\omega_{a b}\right) \mathbf{I} & \omega_{a b} \mathbf{I} \\
\left(\omega_{a}-\omega_{a b}\right) \mathbf{I} & \left(2 \omega_{a b}-\omega_{a}-\omega_{b}\right) \mathbf{I} & \left(\omega_{b}-\omega_{a b}\right) \mathbf{I} \\
\omega_{a b} \mathbf{I} & \left(\omega_{b}-\omega_{a b}\right) \mathbf{I} & -\omega_{b} \mathbf{I}
\end{array}\right]\left\{\begin{array}{l}
\mathbf{u}_{j}^{1} \\
\mathbf{u}_{i}^{1} \\
\mathbf{u}_{k}^{1}
\end{array}\right\}= \\
= & \mathbf{C}_{l}^{1} \mathbf{u}^{1}+\frac{1}{2} \mathbf{u}^{1^{T}} \mathbf{Z}^{1} \mathbf{u}^{1}=\left[\begin{array}{ll}
\mathbf{C}_{l}^{1}+\frac{1}{2} \mathbf{C}_{n}^{1}\left(\mathbf{u}^{1}\right)
\end{array}\right] \mathbf{u}^{1}=\mathbf{C}^{1}\left(\mathbf{u}^{1}\right) \mathbf{u}^{1}
\end{aligned}
$$

donde:

$\mathbf{Z}^{1} \quad$ matriz auxiliar (constante y simétrica).

$\mathbf{C}_{1}^{1} \quad$ matriz cinemática lineal del elemento muelle.

$\mathbf{C}_{n}^{1}\left(\mathbf{u}^{1}\right) \quad$ matriz cinemática no lineal del elemento muelle.

$\mathbf{C}^{1}\left(\mathbf{u}^{1}\right)$ matriz cinemática total del elemento muelle.

Se llega finalmente a la siguiente expresión de la distorsión angular:

$$
e^{1}=\epsilon^{1}+\frac{1}{2} \mathbf{u}^{1 T} \mathbf{Z}^{1} \mathbf{u}^{1}
$$

formalmente idéntica a la ec. (5.7) obtenida para el elemento barra.

\section{Sistema estructural completo}

Una vez obtenidas las ecuaciones cinemáticas no lineales (5.6) y (5.16), y tras compararlas con sus respectivas partes lineales (3.5) y 3.17) se puede concluir que las expresiones cinemáticas de los elementos barra y muelle conservan su estructura, aunque en el caso no lineal las matrices cinemáticas dependerán explícitamente del campo de desplazamientos en cada punto de la trayectoria de equilibrio.

Como consecuencia directa de la analogía anterior, podemos aceptar como válidas para el caso no lineal las reglas de ensamblaje de la matriz cinemática de un sistema estructural incluidas en las ecuaciones (3.26) y 3.27). 


\subsubsection{Ecuaciones de equilibrio}

\section{Elemento barra}

El equilibrio del elemento barra en cualquier punto de la trayectoria de equilibrio no lineal se puede expresar en términos de la ecuación de trabajos virtuales (ETV) para un conjunto de variaciones cinemáticamente admisibles de los desplazamientos nodales $\delta \mathbf{u}_{i}^{a}, \delta \mathbf{u}_{j}^{a}$, como:

$$
\mathbf{f}_{i}^{a T} \delta \mathbf{u}_{i}^{a}+\mathbf{f}_{j}^{a T} \delta \mathbf{u}_{j}^{a}=N^{a} \delta e^{a}
$$

donde $\mathbf{f}_{i}^{a}, \mathbf{f}_{j}^{a}$ son las fuerzas en extremo de barra referidas a la configuración inicial (ver figura 3.2). Puesto que la definición (5.1) está inspirada en el tensor de deformaciones de Green y su variable estática correspondiente es el segundo tensor de tensiones de Piola-Kirchhoff, podemos definir intuitivamente $N^{a}$ como el esfuerzo axil de la barra retrotraído a la configuración de referencia.

Además, podemos evaluar la primera variación $\delta e^{a}$ utilizando la ecuación (5.6) como:

$$
\begin{aligned}
\delta e^{a}=\frac{\partial e^{a}}{\partial \mathbf{u}^{a}} \delta \mathbf{u}^{a}= & {\left[\mathbf{C}_{l}^{a}+\frac{1}{2} \mathbf{u}^{a T} \mathbf{Z}^{a}+\frac{1}{2}\left(\mathbf{Z}^{a} \mathbf{u}^{a}\right)^{T}\right] \delta \mathbf{u}^{a}=} \\
& =\left[\mathbf{C}_{l}^{a}+\mathbf{u}^{a T} \mathbf{Z}^{a}\right] \delta \mathbf{u}^{a}=\left[\mathbf{C}_{l}^{a}+\mathbf{C}_{n}^{a}\left(\mathbf{u}^{a}\right)\right] \delta \mathbf{u}^{a}=\mathbf{b}^{a}\left(\mathbf{u}^{a}\right) \delta \mathbf{u}^{a}
\end{aligned}
$$

donde denotamos por $\mathbf{b}^{a}\left(\mathbf{u}^{a}\right)$ a la matriz cinemática tangente del elemento barra, ya que será la empleada en relaciones cinemáticas de tipo incremental.

Por tanto, sustituyendo en (5.18) y agrupando en bloques:

$$
\begin{gathered}
{\left[\begin{array}{ll}
\mathbf{f}_{i}^{a T} & \mathbf{f}_{j}^{a T}
\end{array}\right]\left\{\begin{array}{l}
\delta \mathbf{u}_{i}^{a} \\
\delta \mathbf{u}_{j}^{a}
\end{array}\right\}=N^{a} \mathbf{b}^{a}\left(\mathbf{u}^{a}\right) \delta \mathbf{u}^{a}} \\
\mathbf{f}^{a T} \delta \mathbf{u}^{a}-N^{a} \mathbf{b}^{a}\left(\mathbf{u}^{a}\right) \delta \mathbf{u}^{a}=\mathbf{0} \\
\mathbf{f}^{a}=N^{a} \mathbf{b}^{a T}\left(\mathbf{u}^{a}\right)=N^{a}\left[\mathbf{C}_{l}^{a T}+\mathbf{C}_{n}^{a T}\left(\mathbf{u}^{a}\right)\right]
\end{gathered}
$$

Por analogía con la ecuación de equilibrio 3.7) de la barra en la configuración de referencia, podemos reescribir 5.20c) como:

$$
\mathbf{f}^{a}=N^{a} \mathbf{H}^{a}\left(\mathbf{u}^{a}\right)=N^{a}\left[\mathbf{H}_{l}^{a}+\mathbf{H}_{n}^{a}\left(\mathbf{u}^{a}\right)\right]
$$

evidentemente se cumplirá:

$$
\mathbf{H}_{l}^{a}=\mathbf{C}_{l}^{a T} \quad \mathbf{H}_{n}^{a}\left(\mathbf{u}^{a}\right)=\mathbf{C}_{n}^{a T}\left(\mathbf{u}^{a}\right) \quad \mathbf{H}^{a}\left(\mathbf{u}^{a}\right)=\mathbf{C}^{a T}\left(\mathbf{u}^{a}\right)
$$

donde:

$\mathbf{H}_{l}^{a} \quad$ matriz de equilibrio lineal del elemento barra.

$\mathbf{H}_{n}^{a}\left(\mathbf{u}^{a}\right) \quad$ matriz de equilibrio no lineal del elemento barra.

$\mathbf{H}^{a}\left(\mathbf{u}^{a}\right)$ matriz de equilibrio total del elemento barra.

\section{Elemento muelle}

Análogamente a la ecuación (5.18), la Ecuación de Trabajos Virtuales (ETV) del elemento muelle en cualquier punto de la trayectoria de equilibrio no lineal se puede escribir para cualquier conjunto de variaciones de los desplazamientos cinemáticamente admisible, como:

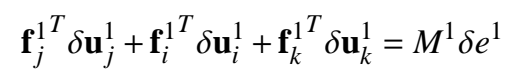

donde $\mathbf{f}_{j}^{1}, \mathbf{f}_{i}^{1}, \mathbf{f}_{k}^{1}$ constituyen la fracción de las fuerzas externas que contribuyen a la distorsión angular $e^{1}$ del elemento, referidas a la configuración de referencia (fig. 3.3). Asimismo, $M^{1}$ es el momento del elemento muelle retrotraído a la geometría inicial. 
También en este caso, la primera variación de la deformación $\delta e^{1}$ se puede evaluar haciendo uso de (5.16):

$$
\begin{aligned}
\delta e^{1}=\frac{\partial e^{1}}{\partial \mathbf{u}^{1}} \delta \mathbf{u}^{1}= & {\left[\mathbf{C}_{l}^{1}+\frac{1}{2} \mathbf{u}^{1 T} \mathbf{Z}^{1}+\frac{1}{2}\left(\mathbf{Z}^{1} \mathbf{u}^{1}\right)^{T}\right] \delta \mathbf{u}^{1}=} \\
& =\left[\mathbf{C}_{l}^{1}+\mathbf{u}^{1^{T}} \mathbf{Z}^{1}\right] \delta \mathbf{u}^{1}=\left[\mathbf{C}_{l}^{1}+\mathbf{C}_{n}^{1}\left(\mathbf{u}^{1}\right)\right] \delta \mathbf{u}^{1}=\mathbf{b}^{1}\left(\mathbf{u}^{1}\right) \delta \mathbf{u}^{1}
\end{aligned}
$$

al igual que para el elemento barra, podemos denominar $\mathbf{b}^{1}\left(\mathbf{u}^{1}\right)$ a la matriz cinemática tangente del elemento muelle.

Sustituyendo en la ETV (5.23) y agrupando matricialmente:

$$
\begin{gathered}
{\left[\begin{array}{ccc}
\mathbf{f}_{j}^{1^{T}} & \mathbf{f}_{i}^{1^{T}} & \mathbf{f}_{k}^{1^{T}}
\end{array}\right]\left\{\begin{array}{l}
\delta \mathbf{u}_{j}^{1} \\
\delta \mathbf{u}_{i}^{1} \\
\delta \mathbf{u}_{k}^{1}
\end{array}\right\}=M^{1} \mathbf{b}^{1}\left(\mathbf{u}^{1}\right) \delta \mathbf{u}^{1}} \\
\mathbf{f}^{1^{T}} \delta \mathbf{u}^{1}-M^{1} \mathbf{b}^{1}\left(\mathbf{u}^{1}\right) \delta \mathbf{u}^{1}=\mathbf{0} \\
\mathbf{f}^{1}=M^{1} \mathbf{b}^{1^{T}}\left(\mathbf{u}^{1}\right)=M^{1}\left[\mathbf{C}_{l}^{1^{T}}+\mathbf{C}_{n}^{1^{T}}\left(\mathbf{u}^{1}\right)\right]
\end{gathered}
$$

Por analogía con la ecuación de equilibrio (3.28) del muelle en la configuración de referencia, podemos reescribir 5.25c como:

$$
\mathbf{f}^{1}=M^{1} \mathbf{H}^{1}\left(\mathbf{u}^{1}\right)=M^{1}\left[\mathbf{H}_{l}^{1}+\mathbf{H}_{n}^{1}\left(\mathbf{u}^{1}\right)\right]
$$

evidentemente se cumplirá:

$$
\mathbf{H}_{l}^{1}=\mathbf{C}_{l}^{1^{T}} \quad \mathbf{H}_{n}^{1}\left(\mathbf{u}^{1}\right)=\mathbf{C}_{n}^{1^{T}\left(\mathbf{u}^{1}\right)} \quad \mathbf{H}^{1}\left(\mathbf{u}^{1}\right)=\mathbf{C}^{1^{T}}\left(\mathbf{u}^{1}\right)
$$

donde:

$\mathbf{H}_{l}^{1} \quad$ matriz de equilibrio lineal del elemento muelle.

$\mathbf{H}_{n}^{1}\left(\mathbf{u}^{1}\right) \quad$ matriz de equilibrio no lineal del elemento muelle.

$\mathbf{H}^{1}\left(\mathbf{u}^{1}\right)$ matriz de equilibrio total del elemento muelle.

\section{Sistema estructural completo}

Como podemos observar, las ecuaciones de equilibrio (5.21) y (5.26) conservan la estructura de las expresiones (3.7) y 3.28) correspondientes al equilibrio en la configuración inicial. Sin embargo, al plantear el equilibrio en un punto genérico de la trayectoria, las matrices de equilibrio implicadas serán funciones de los desplazamientos. Por tanto, aceptamos también las reglas de ensamblaje incluidas en las ecuaciones (3.35) y (3.36) para la matriz de equilibrio en un punto genérico del sistema estructural completo.

Comentario 5.1.1 Como se ha visto, las expresiones variacionales (5.19) y (5.24) han sido necesarias para el desarrollo de las ecuaciones de equilibrio de cada elemento estructural aislado mediante la ETV. Asimismo, veremos más adelante que su versión incremental se empleará en el procedimiento numérico de resolución. Para la estructura completa, tenemos:

$$
\Delta \mathbf{e}=\mathbf{b}(\mathbf{u}) \Delta \mathbf{u}
$$

donde $\mathbf{e}=\left\{\mathbf{e}^{\lambda}, \mathbf{e}^{\Gamma}\right\}^{T}$ contiene el conjunto ordenado de deformaciones no lineales de la estructura. Si introducimos un sistema de desplazamientos impuestos $\Delta \mathbf{u}_{M}=\Delta \overline{\mathbf{u}}=\Delta \lambda \widetilde{\mathbf{d}}$ y teniendo en cuenta 
que las componentes de desplazamiento coaccionadas son nulas $\Delta \mathbf{u}_{A}=\mathbf{0}$, podemos descomponer el miembro derecho de (5.28) (véase (3.37)) según:

$$
\Delta \mathbf{e}=\mathbf{b}_{R}(\mathbf{u}) \Delta \mathbf{u}_{R}+\mathbf{b}_{R M}(\mathbf{u}) \Delta \lambda \widetilde{\mathbf{d}}
$$

donde $\lambda$ es el parámetro de carga, $\Delta \mathbf{u}_{R}$ son los desplazamientos de los nudos libres y $\widetilde{\mathbf{d}}$ es un conjunto conocido de desplazamientos incrementales impuestos que se considerará constante en cada incremento.

\subsection{Ecuaciones constitutivas}

Al igual que en el análisis lineal, compararemos los resultados empleando tanto el potencial AMBER como el Morse (véase 2.4.1 y 4.1.3, ecs. (4.12) a (4.14) para verificar que la no linealidad geométrica se produce antes que la no linealidad mecánica, como se afirma en [27], [43], [94], [110].

\section{Elemento barra}

Si suponemos una función potencial $U$ completamente desacoplada, donde la contribución de cada elemento estructural depende exclusivamente de su propia deformación, podemos establecer la siguiente relación constitutiva general para el elemento barra:

$$
N^{a}=\frac{\partial U}{\partial e^{a}}=f\left(e^{a}\right)
$$

donde $f\left(e^{a}\right)$ es una función no lineal, continua y difereciable (al menos a trozos) de la deformación longitudinal $e^{a}$. En particular, nos centraremos en la ecuación constitutiva en términos incrementales desde un punto de equilibrio dado:

$$
\begin{gathered}
\frac{d N^{a}}{d e^{a}}=f^{\prime}\left(e^{a}\right)=K^{a}\left(e^{a}\right) \\
d N^{a}=K^{a}\left(e^{a}\right) d e^{a}
\end{gathered}
$$

donde $K^{a}$ es la rigidez constitutiva tangente del elemento barra, definida en (4.11). Además, la ecuación (5.31) se puede expresar en términos de la correspondiente variación de Lagrange como:

$$
\delta N^{a}=K^{a}\left(e^{a}\right) \delta e^{a}
$$

\section{Elemento muelle}

Adoptando la misma hipótesis de desacoplamiento de deformaciones sobre el potencial $U$ adoptada en el elemento barra, la ecuación constitutiva general para el elemento muelle tiene una forma:

$$
M^{1}=\frac{\partial U}{\partial e^{1}}=g\left(e^{1}\right)
$$

donde $g\left(e^{1}\right)$ es una función no lineal, continua y diferenciable (al menos a trozos) de la distorsión angular $e^{1}$. Análogamente a lo expuesto en el elemento barra, las ecuaciones constitutivas incrementales y variacionales adoptan la forma:

$$
\begin{gathered}
d M^{1}=g^{\prime}\left(e^{1}\right) d e^{1}=K^{1}\left(e^{1}\right) d e^{1} \\
\delta M^{1}=K^{1}\left(e^{1}\right) \delta e^{1}
\end{gathered}
$$

donde $K^{1}\left(e^{1}\right)$ es la rigidez constitutiva tangente del elemento muelle, definida en (4.11). 


\section{Sistema estructural completo}

Agrupando matricialmente las ecuaciones constitutivas 5.31 y (5.34a de forma adecuada y aproximando los diferenciales por incrementos, la ecuación constitutiva incremental para el sistema estructural completo tiene una expresión:

$$
\left\{\begin{array}{c}
\Delta \mathbf{N} \\
\Delta \mathbf{M}
\end{array}\right\}=\left[\begin{array}{cc}
\mathbf{K}^{\lambda}\left(\mathbf{e}^{\lambda}\right) & \mathbf{0} \\
\mathbf{0} & \mathbf{K}^{\Gamma}\left(\mathbf{e}^{\Gamma}\right)
\end{array}\right]\left\{\begin{array}{c}
\Delta \mathbf{e}^{\lambda} \\
\Delta \mathbf{e}^{\Gamma}
\end{array}\right\}
$$

donde:

$\Delta \mathbf{N} \quad$ vector incremental de axiles de barra retrotraídos a la configuración inicial.

$\Delta \mathbf{M} \quad$ vector incremental de momentos de muelle retrotraídos a la configuración inicial.

$\Delta \mathbf{e}^{\lambda} \quad$ vector incremental de deformaciones longitudinales de barra.

$\Delta \mathbf{e}^{\Gamma} \quad$ vector incremental de distorsiones angulares de muelle.

$\mathbf{K}^{\lambda}\left(\mathbf{e}^{\lambda}\right)$ matriz constitutiva tangente (diagonal) asociada a las barras.

$\mathbf{K}^{\lambda}\left(\mathbf{e}^{\Gamma}\right)$ matriz constitutiva tangente (diagonal) asociada a los muelles.

Por supuesto, la forma variacional de la relación (5.35) se escribe:

$$
\left\{\begin{array}{l}
\delta \mathbf{N} \\
\delta \mathbf{M}
\end{array}\right\}=\left[\begin{array}{cc}
\mathbf{K}^{\lambda}\left(\mathbf{e}^{\lambda}\right) & \mathbf{0} \\
\mathbf{0} & \mathbf{K}^{\Gamma}\left(\mathbf{e}^{\Gamma}\right)
\end{array}\right]\left\{\begin{array}{l}
\delta \mathbf{e}^{\lambda} \\
\delta \mathbf{e}^{\Gamma}
\end{array}\right\}
$$

Comentario 5.2.1 Si el potencial no está completamente desacoplado respecto a cada deformación individual, las matrices constitutivas tangentes incluidas en (5.35) y (5.36) tendrán bloques no nulos fuera de la diagonal. Aunque la formulación energética del modelo MSM (tanto lineal como no lineal geométrica) admite este tipo de potenciales (tipo Tersoff-Brenner), la obtención de dicha matriz constitutiva sería compleja y requeriría probablemente algún criterio de desacoplamiento parcial. Por ejemplo, que las rigideces de los elementos estructurales que salen de un nudo sólo pudieran depender de las posiciones de dicho nudo y de sus adyacentes más cercanos.

Puesto que los potenciales AMBER y Morse (completamente desacoplados) han demostrado ser suficientemente aproximados para los objetivos de la tesis, se ha mantenido también esta hipótesis en el análisis geométricamente no lineal.

\subsubsection{Matriz de rigidez tangente}

El funcional de acción del modelo stick-spiral se define como la energía potencial total del sistema:

$$
F=W_{\text {int }}-W_{\text {ext }}
$$

Consideremos que el valor del funcional viene determinado exclusivamente por un parámetro de carga $\lambda$, entonces para dos configuraciones cualesquiera tenemos:

$$
\begin{aligned}
& F^{1}=W_{i n t}^{1}-W_{e x t}^{1}=F\left(\lambda^{1}\right) \\
& F^{2}=W_{i n t}^{2}-W_{e x t}^{2}=F\left(\lambda^{2}\right)=F\left(\lambda^{1}+\Delta \lambda\right)
\end{aligned}
$$

El incremento del potencial entre dos configuraciones suficientemente próximas se puede desarrollar en serie de Taylor hasta $2^{\circ}$ orden según:

$$
\begin{gathered}
\Delta F=F\left(\lambda^{1}+\Delta \lambda\right)-F\left(\lambda^{1}\right)=\frac{\partial F}{\partial \lambda} \delta \lambda+\frac{1}{2} \frac{\partial^{2} F}{\partial \lambda^{2}} \delta \lambda^{2} \\
\Delta F=\delta F+\frac{1}{2} \delta^{2} F
\end{gathered}
$$


Alrededor de una configuración de equilibrio el potencial deberá ser estacionario, es decir:

$$
\delta F=0 \longrightarrow \delta W_{\text {int }}=\delta W_{\text {ext }}
$$

que es la expresión de la Ecuación de Trabajos Virtuales para cualquier variación cinemáticamente del campo de desplazamientos $\delta \mathbf{u}[56, \S 6.2 .3]$. Por tanto, el incremento (5.40) queda:

$$
\Delta F=\frac{1}{2} \delta^{2} F
$$

Consideremos que las fuerzas externas aumentan proporcionalmente al parámetro de carga $\lambda$ y que este empieza a aumentar desde un valor inicial nulo en la configuración inicial. En estas condiciones, podemos idealizar la respuesta de la estructura como una curva $\mathbf{u}(\lambda)$ (función de $\lambda$ ) que denominamos trayectoria de equilibrio.

Si se produce una pequeña variación del parámetro $\lambda$ el sistema pasará de una configuración de equilibrio a otra, lo cual es equivalente a afirmar que el equilibrio en la primera de ellas es indiferente. Siguiendo [53], la estabilidad del equilibrio viene controlada por (5.42), de forma que la condición de equilibrio indiferente es:

$$
\delta^{2} F=0 \longrightarrow \delta^{2} W_{\text {int }}=\delta^{2} W_{\text {ext }}
$$

Diremos que la expresión anterior define el equilibrio tangente del modelo MSM stick-spiral. Desarrollando el trabajo de las fuerzas internas, se llega a una forma cuadrática en $\delta \mathbf{u}$ cuyo núcleo es la matriz de rigidez tangente:

$$
\delta^{2} W_{i n t}=\delta \mathbf{u}^{T} \mathbf{K}_{T} \delta \mathbf{u}
$$

\section{Elemento barra}

Aplicando la definición (5.44) al elemento barra y empleando (5.32), se llega a:

$$
\delta^{2} W_{\text {int }}=\delta\left(\delta W_{\text {int }}\right)=\delta\left(N^{a} \delta e^{a}\right)=\delta N^{a} \delta e^{a}+N^{a} \delta^{2} e^{a}=K^{a}\left(\delta e^{a}\right)^{2}+N^{a} \delta^{2} e^{a}
$$

Comentario 5.2.2 En el caso más general, la rigidez constitutiva tangente del elemento barra $K^{a}$ es función de los desplazamientos, por tanto $\delta K^{a}\left(e^{a}\right) \neq 0$. Sin embargo, en la expresión anterior hemos supuesto simplificadamente que $K^{a}\left(e^{a}\right)$ es una función constante a trozos, lo que se traduce en $\delta K^{a}\left(e^{a}\right)=0$.

Sustituyendo (5.19), podemos desarrollar (5.45) en la forma:

$$
\delta^{2} W_{\text {int }}=\delta \mathbf{u}^{a T} \mathbf{b}^{a T} K^{a} \mathbf{b}^{a} \delta \mathbf{u}^{a}+N^{a}\left[\delta \mathbf{b}^{a} \delta \mathbf{u}^{a}+\mathbf{b}^{a} \delta^{2} \mathbf{u}^{a}\right]
$$

teniendo en cuenta que la segunda variación de los desplazamientos $\delta^{2} \mathbf{u}^{a}$ se anula, obtenemos directamente la matriz de rigidez tangente para el elemento barra $\mathbf{K}_{T}^{a}$ :

$$
\begin{gathered}
\delta^{2} W_{i n t}=\delta \mathbf{u}^{a T}\left[K^{a} \mathbf{b}^{a T} \mathbf{b}^{a}+N^{a} \mathbf{Z}^{a}\right] \delta \mathbf{u}^{a} \\
\mathbf{K}_{T}^{a}=K^{a} \mathbf{b}^{a T} \mathbf{b}^{a}+N^{a} \mathbf{Z}^{a}
\end{gathered}
$$

Alternativamente, podemos escribir (5.47) como:

$$
\mathbf{K}_{T}^{a}=\mathbf{K}_{M}^{a}\left(\mathbf{u}^{a}\right)+\mathbf{K}_{G}^{a}\left(N^{a}\right)=\left[\begin{array}{cc}
\mathbf{K}_{i i}^{a} & \mathbf{K}_{i j}^{a} \\
\mathbf{K}_{j i}^{a} & \mathbf{K}_{j j}^{a}
\end{array}\right]
$$

donde habitualmente [22],[23] $\mathbf{K}_{M}^{a}$ se denomina matriz de rigidez material y depende explícitamente del bloque de desplazamientos. Por otra parte, $\mathbf{K}_{G}^{a}$ se denomina matriz de rigidez geométrica y depende del axil de la barra. Como se puede observar, la descomposición en bloques final está asociada a los nudos que conecta el elemento barra. 


\section{Elemento muelle}

Se adopta la definición (5.44) al elemento muelle para obtener su matriz de rigidez tangente. Empleando (5.34b) y adoptando la simplificación expuesta en el comentario 5.2.2.

$$
\delta^{2} W_{i n t}=\delta\left(\delta W_{i n t}\right)=\delta\left(M^{1} \delta e^{1}\right)=\delta M^{1} \delta e^{1}+M^{1} \delta^{2} e^{1}=K^{1}\left(\delta e^{1}\right)^{2}+M^{1} \delta^{2} e^{1}
$$

Sustituyendo (5.24) y teniendo en cuenta que la segunda variación de los desplazamientos $\delta^{2} \mathbf{u}^{1}$ se anula, obtenemos la matriz de rigidez tangente $\mathbf{K}_{T}^{1}$ del elemento muelle:

$$
\begin{gathered}
\delta^{2} W_{\text {int }}=\delta \mathbf{u}^{1^{T}}\left[K^{1} \mathbf{b}^{1^{T}} \mathbf{b}^{1}+M^{1} \mathbf{Z}^{1}\right] \delta \mathbf{u}^{1} \\
\mathbf{K}_{T}^{1}=K^{1} \mathbf{b}^{{ }^{T}} \mathbf{b}^{1}+M^{1} \mathbf{Z}^{1}
\end{gathered}
$$

Además, (5.50) puede reescribirse de igual forma que se hizo para el elemento barra:

$$
\mathbf{K}_{T}^{1}=\mathbf{K}_{M}^{1}\left(\mathbf{u}^{1}\right)+\mathbf{K}_{G}^{1}\left(M^{1}\right)=\left[\begin{array}{ccc}
\mathbf{K}_{j j}^{1} & \mathbf{K}_{j i}^{1} & \mathbf{K}_{j k}^{1} \\
\mathbf{K}_{i j}^{1} & \mathbf{K}_{i i}^{1} & \mathbf{K}_{i k}^{1} \\
\mathbf{K}_{k j}^{1} & \mathbf{K}_{k i}^{1} & \mathbf{K}_{k k}^{1}
\end{array}\right]
$$

donde $\mathbf{K}_{M}^{1}$ es la matriz de rigidez material y depende explícitamente del bloque de desplazamientos. Por otra parte, $\mathbf{K}_{G}^{1}$ es la matriz de rigidez geométrica y depende del momento del elemento muelle. Como se puede observar, la descomposición en bloques final está asociada a los nudos implicados en el elemento muelle.

\section{Unidad básica}

Consideremos en este epígrafe la unidad básica esquematizada en la figura 3.4 si aplicamos la definición (5.44) llegamos a:

$$
\delta^{2} W_{\text {int }}=\delta\left(\delta W_{\text {int }}\right)=\delta\left(N^{a} \delta e^{a}\right)+\delta\left(N^{b} \delta e^{b}\right)+\delta\left(M^{1} \delta e^{1}\right)
$$

Por tanto la segunda variación $\delta^{2} W_{\text {int }}$ queda descompuesta en la suma de los términos asociados a los elementos incluidos en la unidad básica. Haciendo uso apropiadamente de las descomposiciones en bloques (5.48) y (5.51) queda:

$$
\begin{aligned}
& \delta^{2} W_{i n t}=\left[\begin{array}{ll}
\delta \mathbf{u}_{i}^{T} & \delta \mathbf{u}_{j}^{T}
\end{array}\right]\left[\begin{array}{cc}
\mathbf{K}_{i i}^{a} & \mathbf{K}_{i j}^{a} \\
\mathbf{K}_{j i}^{a} & \mathbf{K}_{j j}^{a}
\end{array}\right]\left\{\begin{array}{c}
\delta \mathbf{u}_{i} \\
\delta \mathbf{u}_{j}
\end{array}\right\}+\left[\begin{array}{ll}
\delta \mathbf{u}_{i}^{T} & \delta \mathbf{u}_{k}^{T}
\end{array}\right]\left[\begin{array}{cc}
\mathbf{K}_{i i}^{b} & \mathbf{K}_{i k}^{b} \\
\mathbf{K}_{k i}^{b} & \mathbf{K}_{k k}^{b}
\end{array}\right]\left\{\begin{array}{c}
\delta \mathbf{u}_{i} \\
\delta \mathbf{u}_{k}
\end{array}\right\}+ \\
& +\left[\begin{array}{lll}
\delta \mathbf{u}_{j}^{T} & \delta \mathbf{u}_{i}^{T} & \delta \mathbf{u}_{j}^{T}
\end{array}\right]\left[\begin{array}{ccc}
\mathbf{K}_{j j}^{1} & \mathbf{K}_{j i}^{1} & \mathbf{K}_{j k}^{1} \\
\mathbf{K}_{i j}^{1} & \mathbf{K}_{i i}^{1} & \mathbf{K}_{i k}^{1} \\
\mathbf{K}_{k j}^{1} & \mathbf{K}_{k i}^{1} & \mathbf{K}_{k k}^{1}
\end{array}\right]\left\{\begin{array}{c}
\delta \mathbf{u}_{j} \\
\delta \mathbf{u}_{i} \\
\delta \mathbf{u}_{k}
\end{array}\right\}= \\
& \left.=\left[\begin{array}{lll}
\delta \mathbf{u}_{j}^{T} & \delta \mathbf{u}_{i}^{T} & \delta \mathbf{u}_{j}^{T}
\end{array}\right]\left[\begin{array}{ccc}
\mathbf{K}_{j j}^{a} & \mathbf{K}_{j i}^{a} & \mathbf{0} \\
\mathbf{K}_{i j}^{a} & \mathbf{K}_{i i}^{a} & \mathbf{0} \\
\mathbf{0} & \mathbf{0} & \mathbf{0}
\end{array}\right]+\left[\begin{array}{ccc}
\mathbf{0} & \mathbf{0} & \mathbf{0} \\
\mathbf{0} & \mathbf{K}_{i i}^{b} & \mathbf{K}_{i k}^{b} \\
\mathbf{0} & \mathbf{K}_{k i}^{b} & \mathbf{K}_{k k}^{b}
\end{array}\right]+\left[\begin{array}{ccc}
\mathbf{K}_{j j}^{1} & \mathbf{K}_{j i}^{1} & \mathbf{K}_{j k}^{1} \\
\mathbf{K}_{i j}^{1} & \mathbf{K}_{i i}^{1} & \mathbf{K}_{i k}^{1} \\
\mathbf{K}_{k j}^{1} & \mathbf{K}_{k i}^{1} & \mathbf{K}_{k k}^{1}
\end{array}\right]\right]\left\{\begin{array}{c}
\delta \mathbf{u}_{j} \\
\delta \mathbf{u}_{i} \\
\delta \mathbf{u}_{k}
\end{array}\right\}= \\
& =\delta \mathbf{u}^{T} \mathbf{K}_{T} \delta \mathbf{u}
\end{aligned}
$$

donde hemos obtenido una descomposición en bloques de la matriz de rigidez tangente de la unidad como sigue:

$$
\mathbf{K}_{T}=\left[\begin{array}{ccc}
\mathbf{K}_{j j}^{a}+\mathbf{K}_{j j}^{1} & \mathbf{K}_{j i}^{a}+\mathbf{K}_{j i}^{1} & \mathbf{K}_{j k}^{1} \\
\mathbf{K}_{i j}^{a}+\mathbf{K}_{i j}^{1} & \mathbf{K}_{i i}^{a}+\mathbf{K}_{i i}^{b}+\mathbf{K}_{i i}^{1} & \mathbf{K}_{i k}^{b}+\mathbf{K}_{i k}^{1} \\
\mathbf{K}_{k j}^{1} & \mathbf{K}_{k i}^{b}+\mathbf{K}_{k i}^{1} & \mathbf{K}_{k k}^{b}+\mathbf{K}_{k k}^{1}
\end{array}\right]
$$


En definitiva, la deducción del ensamblaje booleano para el análisis lineal expresada en (4.21) se puede extender de forma consistente a la matriz de rigidez tangente en el análisis geométricamente no lineal.

\section{Sistema estructural completo}

A continuación se obtiene una expresión compacta del sistema incremental de rigidez para cualquier conjunto de elementos barra y muelle ensamblados adecuadamente. En particular, se tratan aquellos conjuntos de elementos que reproducen la geometría de los SWNTs.

Agrupando por bloques la matriz de rigidez tangente del sistema completo ensamblada siguiendo (5.54), el sistema de rigidez incremental correspondiente (desde una configuración genérica de equilibrio) se puede escribir en la forma:

$$
\left\{\begin{array}{c}
\Delta \mathbf{f}_{R} \\
\Delta \mathbf{f}_{A} \\
\Delta \mathbf{f}_{M}
\end{array}\right\}=\left[\begin{array}{ccc}
\mathbf{K}_{R} & \mathbf{K}_{R A} & \mathbf{K}_{R M} \\
\mathbf{K}_{A R} & \mathbf{K}_{A} & \mathbf{K}_{A M} \\
\mathbf{K}_{M R} & \mathbf{K}_{M A} & \mathbf{K}_{M}
\end{array}\right]\left\{\begin{array}{c}
\Delta \mathbf{u}_{R} \\
\Delta \mathbf{u}_{A} \\
\Delta \mathbf{u}_{M}
\end{array}\right\}
$$

Efectuamos la reducción del sistema incremental anterior teniendo en cuenta que $\Delta \mathbf{u}_{A}=\mathbf{0}$ en los apoyos fijos:

$$
\left\{\begin{array}{c}
\Delta \mathbf{f}_{R} \\
\Delta \mathbf{f}_{M}
\end{array}\right\}=\left[\begin{array}{cc}
\mathbf{K}_{R} & \mathbf{K}_{R M} \\
\mathbf{K}_{M R} & \mathbf{K}_{M}
\end{array}\right]\left\{\begin{array}{c}
\Delta \mathbf{u}_{R} \\
\Delta \mathbf{u}_{M}
\end{array}\right\}
$$

Desarrollando la primera banda matricial de (5.56) e introduciendo un conjunto conocido de funciones de desplazamiento impuesto en los apoyos móviles $\Delta \mathbf{u}_{M}=\Delta \overline{\mathbf{u}}$, se llega a:

$$
\Delta \mathbf{f}_{R}-\mathbf{K}_{R M} \Delta \overline{\mathbf{u}}=\mathbf{K}_{R} \Delta \mathbf{u}_{R}
$$

Tal como se mencionó anteriormente, las cargas externas y los desplazamientos impuestos crecen proporcionalmente a un factor de carga $\lambda$, la ec. (5.57) se puede reescribir como2:

$$
\begin{gathered}
\Delta \mathbf{f}_{R}-\mathbf{K}_{R M} \Delta \overline{\mathbf{u}}=\Delta \lambda \mathbf{q}_{\epsilon}+\Delta \lambda \overline{\mathbf{q}}=\mathbf{K}_{R} \Delta \mathbf{u}_{R} \\
\Delta \lambda \mathbf{q}=\Delta \lambda\left(\mathbf{q}_{\epsilon}+\overline{\mathbf{q}}\right)=\mathbf{K} \Delta \mathbf{u}
\end{gathered}
$$

donde $\overline{\mathbf{q}}=-\mathbf{K}_{R M} \widetilde{\mathbf{d}}$ (ver comentario 5.1.1) es el denominado vector de carga incremental equivalente a los desplazamientos impuestos y $\mathbf{q}_{\epsilon}$ es el vector de imperfección incremental introducido al inicio del proceso de carga para atravesar los puntos críticos (de bifurcación) que aparezcan en la trayectoria de equilibrio. En adelante, el vector de carga incremental q será considerado constante.

\subsection{Implementación numérica}

Con el propósito de investigar las deformaciones y/o tensiones críticas en el comportamiento estructural de SWNTs bajo varios casos de carga, se ha implementado numéricamente el trazado de trayectorias de equilibrio no lineales mediante los denominados métodos de continuación. De hecho, se han desarrollado procedimientos específicos de tipo incremental-iterativo [29] (o predictorcorrector), los cuales pretenden encontrar una nueva configuración de equilibrio partiendo desde una situación de equilibrio conocida, dentro de cada incremento en que se descompone la trayectoria final.

\footnotetext{
${ }^{2}$ se han omitido los subíndices en $[5.58$ por simplicidad en la notación
} 


\subsubsection{Planteamiento del problema numérico}

En esta sección se emplea el planteamiento de Felippa [29] para desarrollar el algoritmo numérico de resolución, asumiendo que todas las variables de estado (desplazamientos, deformaciones, esfuerzos, fuerzas externas y desplazamientos impuestos) crecen en proporción al factor de carga $\lambda$. Descomponemos la trayectoria de equilibrio resultante en $N I$ incrementos y aceptamos que se han finalizado $n$ incrementos del análisis hasta la última solución aceptada $\mathbf{u}_{n}, \lambda_{n}$. En esta situación, buscamos obtener la próxima solución del equilibrio

$$
\begin{aligned}
& \mathbf{u}_{n+1}=\mathbf{u}_{n}+\Delta \mathbf{u}_{n} \\
& \lambda_{n+1}=\lambda_{n}+\Delta \lambda_{n}
\end{aligned}
$$

que satisfaga el sistema no lineal:

$$
\begin{array}{r}
\mathbf{r}\left(\mathbf{u}_{n+1}, \lambda_{n+1}\right)=\mathbf{0} \\
c\left(\Delta \mathbf{u}_{n}, \Delta \lambda_{n}\right)=0
\end{array}
$$

donde $\mathbf{r}$ es el residuo del problema no lineal y $c$ es la ecuación de restricción asociada a la estrategia de control. A partir de aquí, el subíndice $n$ se refiere al incremento actual y el superíndice $k$ está relacionado con la presente iteración dentro de cada incremento.

La forma general de la ecuación residual [29] es:

$$
\mathbf{r}(\mathbf{u}, \lambda)=\mathbf{K}(\mathbf{u}) \mathbf{u}-\lambda \mathbf{q}
$$

En nuestro caso, se adopta una estrategia de control de arco (arc-length), ya que es capaz de atravesar los puntos límite que aparecerán sobre la trayectoria de equilibrio, contrariamente al control de fuerza [29]. Representamos en la figura 5.1] un esquema de las variables implicadas en la restricción $5.59 \mathrm{~b}$. Conviene señalar que la gráfica es una proyección ideal de un espacio multidimensional en la trayectoria que resultaría de un problema 1D para posibilitar la representación. No obstante, las definiciones incluidas en ella son completamente generales.

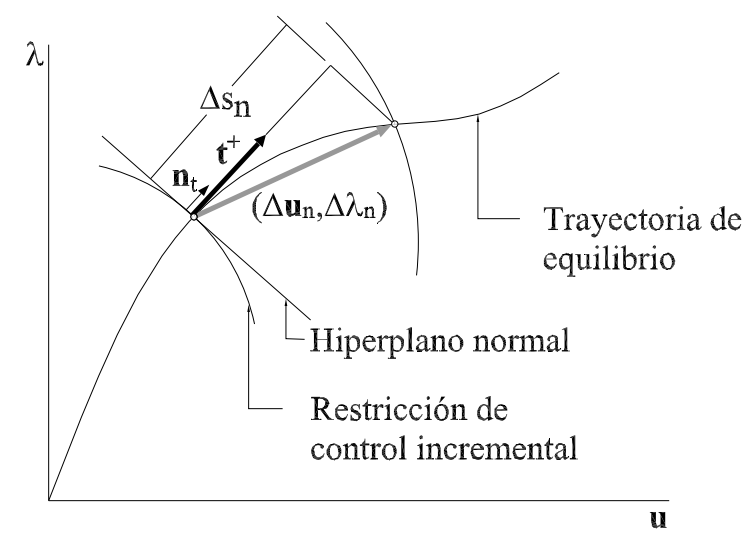

Figura 5.1: Esquema de la estrategia de control en cada incremento

En cualquier punto regular (aunque no necesariamente sobre la trayectoria de equilibrio) se puede definir el vector tangente en el sentido positivo de recorrido de la curva por:

$$
\mathbf{t}^{+}=\left[\begin{array}{l}
\mathbf{u}^{\prime} \\
\lambda^{\prime}
\end{array}\right]=\left[\begin{array}{l}
\mathbf{v} \\
1
\end{array}\right]
$$


donde ( )' denota derivación respecto a $\lambda$ y $\mathbf{v}$ representa el vector velocidad incremental, el cual se puede evaluar en un punto de equilibrio a partir de (5.60) como:

$$
\mathbf{v}=\mathbf{K}^{-1}(\mathbf{u}) \mathbf{q}
$$

además, podemos definir el vector tangente normalizado $\mathbf{n}_{t}$ como:

$$
\mathbf{n}_{t}=\left[\begin{array}{l}
\mathbf{v} / f \\
1 / f
\end{array}\right]
$$

donde f es el factor de escala:

$$
f=\sqrt{1+\mathbf{v}^{T} \mathbf{v}}
$$

Si proyectamos a continuación el vector incremento $\Delta \mathbf{u}_{n}, \Delta \lambda_{n}$ sobre la dirección tangente a la trayectoria, la longitud (aproximada) de arco desde una posición de equilibrio genérico adopta la siguiente expresión:

$$
\Delta s_{n}=\frac{1}{f_{n}}\left(\mathbf{v}_{n}^{T} \Delta \mathbf{u}_{n}+\Delta \lambda_{n}\right)
$$

Esta expresión cobrará especial significado cuando se supone un tamaño de incremento suficientemente pequeño. Si tomamos ahora $\Delta s_{n}=l_{0}$, tenemos:

$$
c\left(\Delta \mathbf{u}_{n}, \Delta \lambda_{n}\right)=\mathbf{v}_{n}^{T} \Delta \mathbf{u}_{n}+\Delta \lambda_{n}-l_{0} f_{n}=0
$$

Los métodos tipo Newton se basan en el desarrollo en serie de Taylor hasta el primer orden del sistema (5.59) alrededor de $\mathbf{u}_{n}^{k}, \lambda_{n}^{k}$. De esta forma, empleando las ecs. (5.60) y (5.66):

$$
\begin{aligned}
\mathbf{r}_{n}^{k+1} & =\mathbf{r}_{n}^{k}+\mathbf{K}\left(\mathbf{u}_{n}^{k}\right) \Delta \mathbf{u}_{n}^{k}-\mathbf{q}_{n}^{k} \Delta \lambda_{n}^{k} \\
c_{n}^{k+1} & =c_{n}^{k}+\mathbf{v}_{n}^{k^{T}} \Delta \mathbf{u}_{n}^{k}+\Delta \lambda_{n}^{k}
\end{aligned}
$$

Dado que $\mathbf{r}_{n}^{k+1}, c_{n}^{k+1}$ se anulará en la convergencia de la solución a (5.59), denotando $\mathbf{d}_{n}^{k}=\Delta \mathbf{u}_{n}^{k}, \eta_{n}^{k}=$ $\Delta \lambda_{n}^{k}$ el sistema ampliado de rigidez viene dado por:

$$
\left[\begin{array}{cc}
\mathbf{K}\left(\mathbf{u}_{n}^{k}\right) & -\mathbf{q}_{n}^{k} \\
\mathbf{v}_{n}^{k^{T}} & 1
\end{array}\right]\left\{\begin{array}{l}
\mathbf{d}_{n}^{k} \\
\eta_{n}^{k}
\end{array}\right\}=-\left\{\begin{array}{l}
\mathbf{r}_{n}^{k} \\
c_{n}^{k}
\end{array}\right\}
$$

donde las variables implicadas se han definido previamente para el sistema estructural completo. Nótese que (5.68) es el sistema de ecuaciones fundamental para la fase correctiva, aunque la evaluación de $\mathbf{r}_{n}^{k}, c_{n}^{k}$ en la primera iteración requiere una estimación inicial de $\mathbf{u}_{n}^{1}, \lambda_{n}^{1}$ en la fase predictiva. Éstos valores se han obtenido mediante los siguientes pasos:

1. La última solución aceptada es el punto de partida del incremento actual $\mathbf{u}_{n}^{0}, \lambda_{n}^{0}$

2. Cálculo de la velocidad incremental inicial $\mathbf{v}_{n}^{0}$ mediante (5.62)

3. Estimación inicial de $\Delta \lambda_{n}$ :

$$
\Delta \lambda_{n}^{0}=\frac{l_{0}}{f_{n}^{0}}
$$

4. Evaluación del incremento inicial de desplazamientos:

$$
\Delta \mathbf{u}_{n}^{0}=\Delta \lambda_{n}^{0} \mathbf{v}_{n}^{0}
$$

5. Estimación inicial:

$$
\mathbf{u}_{n}^{1}=\mathbf{u}_{n}^{0}+\Delta \mathbf{u}_{n}^{0} \quad \lambda_{n}^{1}=\lambda_{n}^{0}+\Delta \lambda_{n}^{0}
$$




\subsubsection{Descripción del proceso incremental-iterativo}

Basado en el planteamiento numérico descrito, se ha implementado numéricamente el problema empleando VisualLISP para la generación de la geometría y la salida de resultados gráficos, y desarrollando el núcleo de cálculo en lenguaje $\mathrm{C}++$. Representamos en la figura 5.2 el diagrama de flujo general del proceso empleado, y en las figuras 5.3(a) y 5.3(b) diagramas más detallados para las fases predictiva y correctiva respectivamente. Las ecuaciones implicadas se han referenciado adecuadamente.

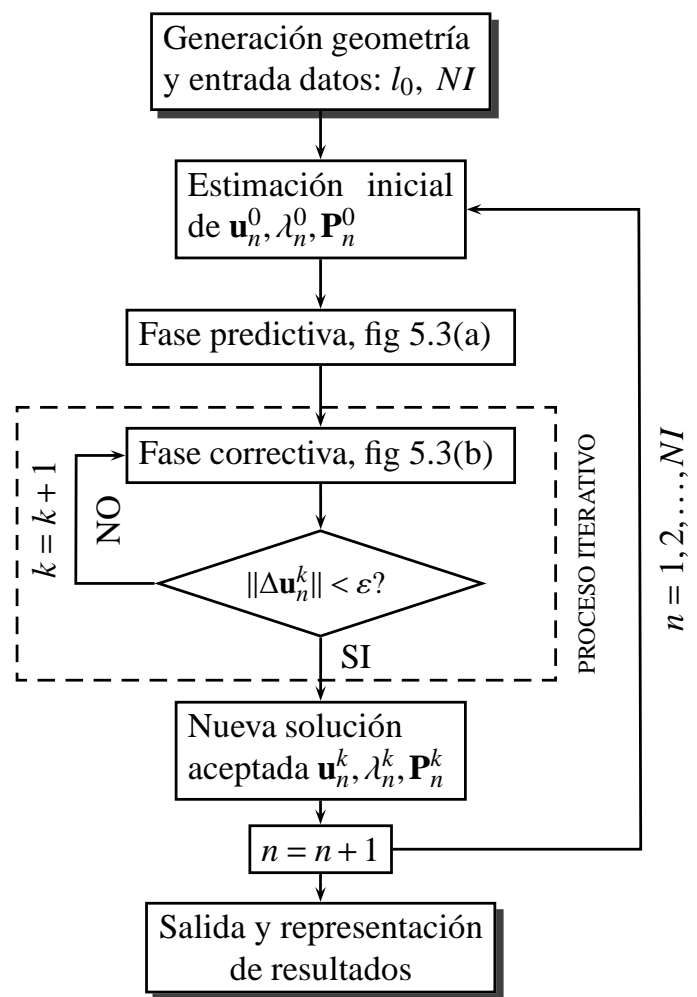

Figura 5.2: Diagrama de flujo del proceso incremental-iterativo

Para chequear la influencia del potencial interatómico en la respuesta geométricamente no lineal de SWNTs se han desarrollado dos códigos específicos. Uno de ellos emplea las rigideces tangentes del potencial AMBER (4.12b) y el otro las del potencial Morse 4.14) en las ecuaciones constitutivas incrementales, similares a (5.35).

Como se vio en el capítulo 4, la preenergía no produce diferencias significativas en la rigidez del nanotubo frente a los diferentes casos de carga allí estudiados. Si bien es cierto que se prevé cierto efecto estabilizador frente a los distintos modos de inestabilidad, éste sólo será notable en los nanotubos más pequeños (inusuales experimentalmente) y se disipará acusadamente con el aumento del diámetro. Por tanto, el pretensado no se ha incluido en el diagrama de flujo (fig. 5.2) y se asume la configuración inicial para el primer incremento como $\mathbf{u}_{1}^{0}=\mathbf{0}, \lambda_{1}^{0}=0, \mathbf{P}_{1}^{0}=\mathbf{0}$. No obstante, la introducción de esfuerzos iniciales en el análisis geométricamente no lineal de SWNTs mediante el modelo stick-spiral es factible y los primeros tanteos en esa línea de investigación se incluyen en el capítulo 7

En virtud de una mayor claridad en la exposición de los diagramas detallados en las figuras 5.3(a) y 5.3(b) añadimos los siguientes comentarios: 


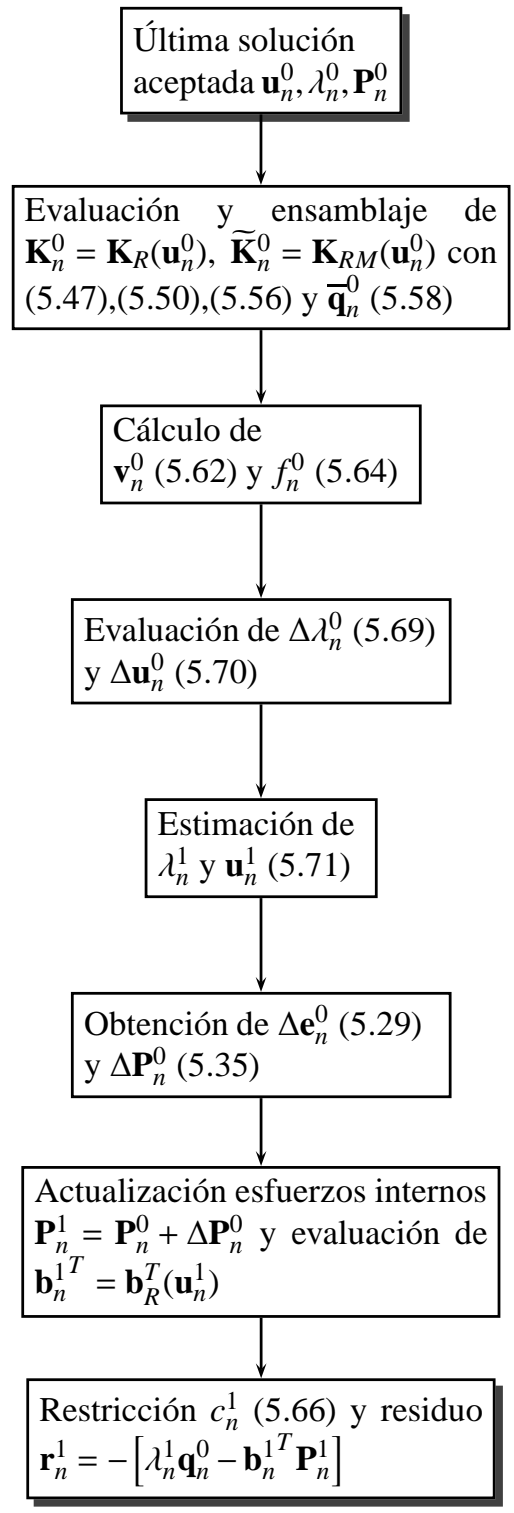

(a) Fase predictiva (incremental)

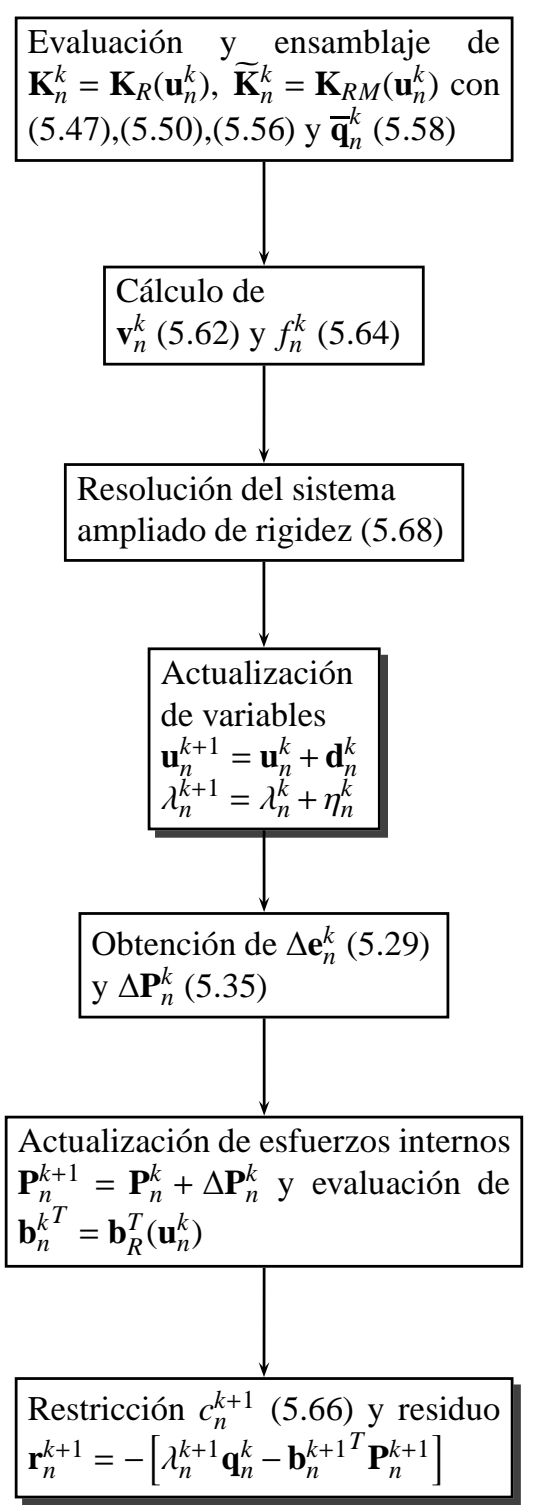

(b) Fase correctiva (iterativa)

Figura 5.3: Descomposición de un incremento genérico

1. Los datos de entrada serán el tamaño del incremento o longitud de arco $l_{0}$ y el número de incrementos a representar sobre la trayectoria de equilibrio no lineal. Se ha procedido por tanteos en cada simulación hasta obtener la combinación óptima de estos valores que reproduce el comportamiento postcrítico más largo antes de la divergencia del proceso numérico. Como resultados de salida, hemos obtenido la correspondiente trayectoria de equilibrio y la situación final para el último incremento (geometría deformada, esfuerzos y reacciones).

2. Aunque se puede adoptar una condición de convergencia sobre el residuo, se prefirió adoptar 
una condición de convergencia sobre los desplazamientos (fig. 5.3(b) tomando $\varepsilon=10^{-6}$ como cota de error absoluto. Por otra parte, la norma escogida para el incremento de desplazamientos es la norma del máximo.

3. El vector $\mathbf{P}=\{\mathbf{N}, \mathbf{M}\}^{T}$ contiene los esfuerzos internos del sistema estructural completo, donde $\mathbf{N}$ son los axiles de barra y $\mathbf{M}$ son los momentos de muelle. Análogamente, $\mathbf{e}=\left\{\mathbf{e}^{\lambda}, \mathbf{e}^{\Gamma}\right\}^{T}$ corresponde al vector no lineal de deformaciones, donde $\mathbf{e}^{\lambda}$ son las deformaciones longitudinales y $\mathbf{e}^{\Gamma}$ las angulares.

4. Respecto al vector de carga incremental $\mathbf{q}=\mathbf{q}_{\epsilon}+\overline{\mathbf{q}}$, el primer término (imperfección inicial) se introduce exclusivamente en el primer incremento para atravesar los puntos críticos que aparecerán en la trayectoria de equilibrio y deberá eliminarse de q en los siguientes. Además, el segundo término se calcula mediante (ver ec. (5.58)):

$$
\begin{gathered}
\Delta \overline{\mathbf{u}}=\Delta \lambda \widetilde{\mathbf{d}} \\
\Delta \lambda \overline{\mathbf{q}}=-\Delta \lambda \mathbf{K}_{R M} \widetilde{\mathbf{d}} \\
\overline{\mathbf{q}}=-\mathbf{K}_{R M} \widetilde{\mathbf{d}}=-\widetilde{\mathbf{K}} \widetilde{\mathbf{d}}
\end{gathered}
$$

donde se denomina $\widetilde{\mathbf{d}}$ al conjunto de desplazamientos impuestos incrementales en los apoyos móviles. Ya que el primer término del residuo sólo incluye las cargas externas que actúan realmente y no las pseudo-cargas $\overline{\mathbf{q}}$, dicho término ha sido suprimido del residuo a partir del segundo incremento.

5. Como veremos más adelante, los desplazamientos impuestos incrementales $\widetilde{\mathbf{d}}_{n}$ se consideran constantes en cada incremento, de modo que no se actualizan en la fase correctiva para permitir al modelo MSM resdistribuir esfuerzos y deformaciones hasta alcanzar la siguiente configuración de equilibrio. Mediante esta técnica se obtiene una mayor velocidad de convergencia.

6. Cabe hacer notar que los incrementos $\Delta \mathbf{u}_{n}, \Delta \lambda_{n}$ incluidos en la restricción (5.66) se deben tomar desde el inicio de cada incremento, no entre dos iteraciones consecutivas.

\subsection{Resultados numéricos y discusión}

En esta sección, se analiza el comportamiento geométricamente no lineal de nanotubos mediante simulaciones a compresión, flexión y torsión de SWNTs. En todos los casos, se ha adoptado una configuración de apoyos tipo viga biempotrada, es decir, se han coaccionado los desplazamientos de todos los nodos del extremo izquierdo mediante un conjunto de apoyos fijos y se ha cargado el nanotubo introduciendo una corona de apoyos móviles (dotados de ciertas funciones de desplazamiento impuesto) en el extremo derecho.

El principal objetivo del análisis es obtener el factor de carga crítico para cada esquema de carga y comparar los resultados finales con algunas simulaciones atómicas disponibles en la literatura.

\subsubsection{Comportamiento a compresión}

Los nanotubos ensayados a compresión se detallan en la tabla 5.1. Se adoptaron diámetros y esbelteces similares para facilitar la comparación posterior de resultados. Las condiciones de sustentación y el esquema de desplazamientos impuestos a compresión se representan en la figura 5.4 De ahí que la función de desplazamientos impuestos adoptada en cada apoyo articulado del extremo 


\begin{tabular}{lccc}
\hline Quiralidad & $\mathrm{d}(\mathrm{nm})$ & $\mathrm{L}(\mathrm{nm})$ & $\mathrm{L} / \mathrm{d}$ \\
\hline $\mathrm{AC}(3,3)$ & 0.407 & 4.919 & 12.09 \\
$\mathrm{AC}(4,4)$ & 0.542 & 6.641 & 12.24 \\
\hline $\mathrm{ZZ}(5,0)$ & 0.391 & 4.757 & 12.15 \\
$\mathrm{ZZ}(7,0)$ & 0.548 & 6.674 & 12.18 \\
\hline $\mathrm{CH}(4,2)$ & 0.414 & 5.072 & 12.24 \\
$\mathrm{CH}(5,3)$ & 0.548 & 6.859 & 12.52 \\
\hline
\end{tabular}

Tabla 5.1: SWNTs ensayados a compresión

derecho del SWNT sea:

$$
\Delta \overline{\mathbf{u}}_{n}=\Delta \lambda_{n}\left\{\begin{array}{c}
-1 \\
0 \\
0
\end{array}\right\}
$$

Puesto que el eje $x$ del sistema de referencia cartesiano definido en la figura 5.4 es paralelo al eje longitudinal del cilindro, $\Delta \lambda_{n}$ corresponde directamente con el acortamiento del nanotubo en cada incremento y la ecuación (5.73) constituye una traslación de sólido rígido de la sección transversal del extremo derecho hacia el izquierdo.

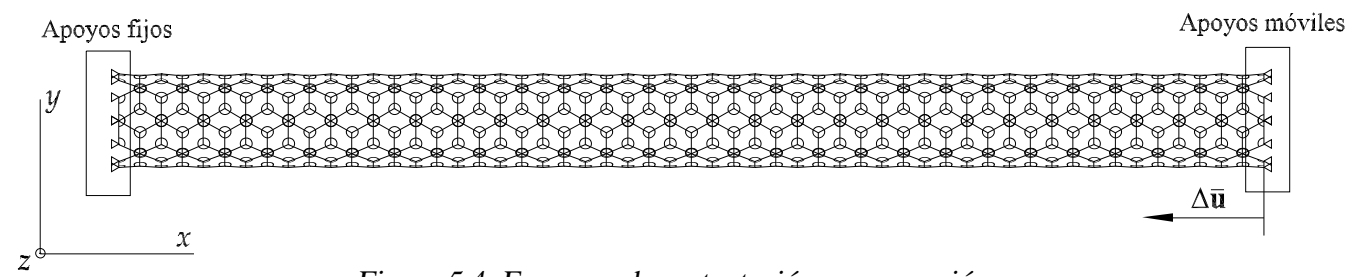

Figura 5.4: Esquema de sustentación a compresión

Se han efectuado dos análisis para cada nanotubo de la tabla 5.1 a compresión (potenciales AMBER y Morse). Puesto que la representación más sencilla para las trayectorias de equilibrio no lineales son gráficas 2D, adoptamos como variable fundamental el desplazamiento transversal $u_{\rho}$ de un nodo representativo alrededor del punto medio del SWNT para estudiar su variación respecto al factor de carga $\lambda$. Las curvas resultado del análisis vienen dadas en la figura 5.5 y algunas deformadas finales se proporcionan en la figura 5.6 como ejemplos del comportamiento a pandeo obtenido.

Respecto a la configuración de las imperfecciones iniciales, en todas las simulaciones a compresión hemos adoptado un conjunto de cargas transversales puntuales alrededor del punto medio del nanotubo, cuyos valores se han tanteado para conseguir reproducir una respuesta postcrítica más extensa sin divergencia del algoritmo numérico.

Comentario 5.4.1 Las imperfecciones iniciales solo se introducen en el primer incremento del proceso numérico (ver sección 5.3) y su valor viene determinado por la facilidad que proporcionan al algoritmo numérico en atravesar los puntos críticos que aparecen en la trayectoria. En consecuencia, en algunos casos se requiere introducir imperfecciones de valor algo superior al deseable, que provocan una pequeña perturbación localizada en el inicio de la trayectoria de equilibrio, como puede verse claramente en las figs. 5.5(b) y $5.5(d)$ 


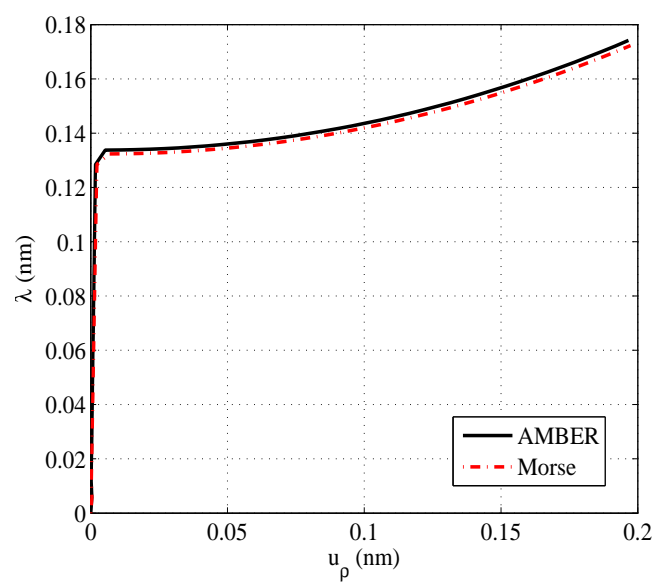

(a) $\mathrm{ZZ}(5,0)$ compresión

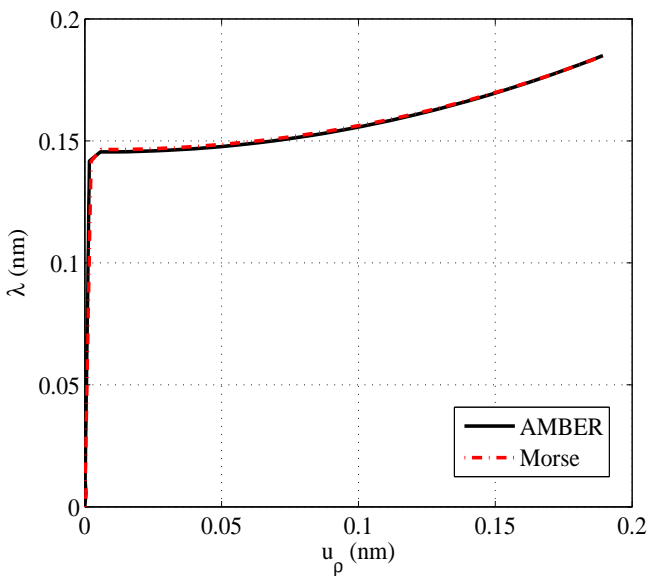

(c) $\mathrm{AC}(3,3)$ compresión

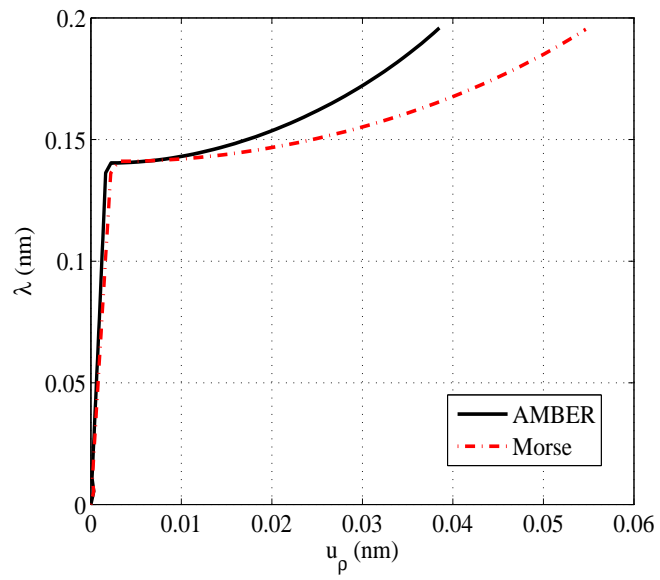

(e) $\mathrm{CH}(4,2)$ compresión

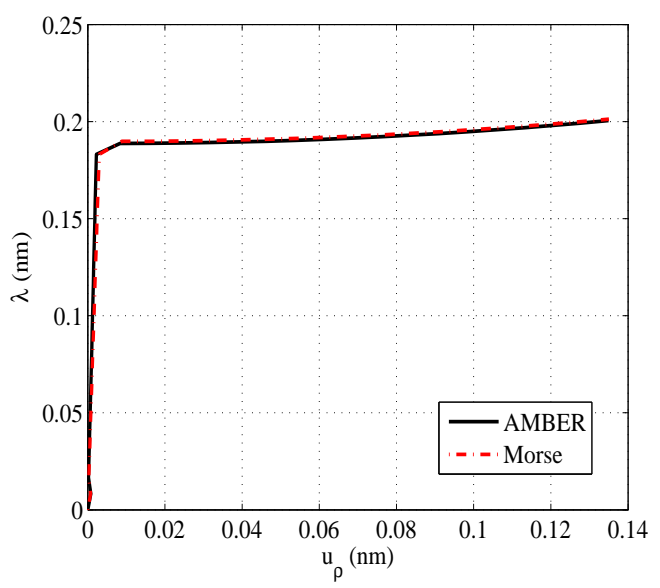

(b) ZZ(7,0) compresión

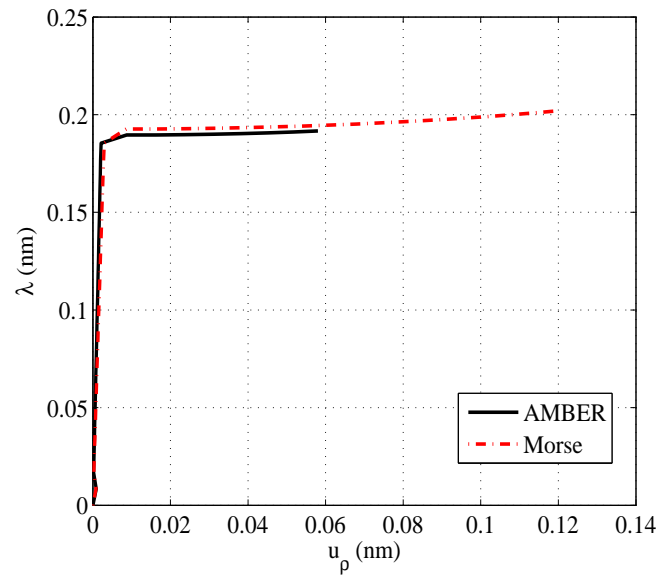

(d) $\mathrm{AC}(4,4)$ compresión

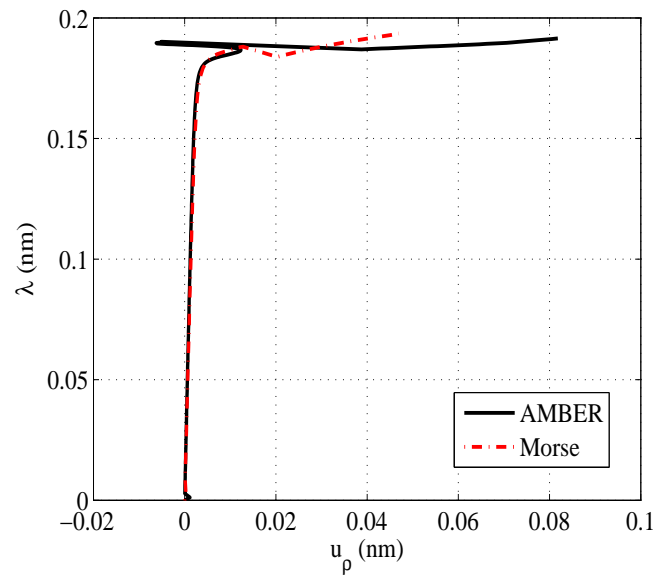

(f) $\mathrm{CH}(5,3)$ compresión

Figura 5.5: Trayectorias no lineales $S W N T$ s compresión 


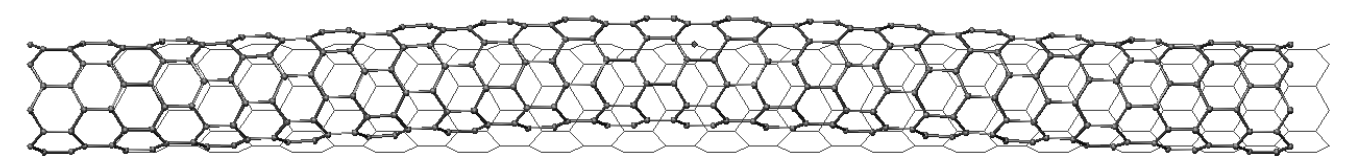

(a) ZZ(7,0) AMBER

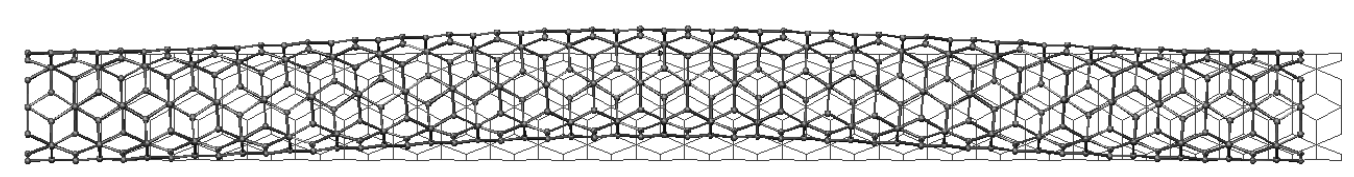

(b) $\mathrm{AC}(4,4)$ Morse

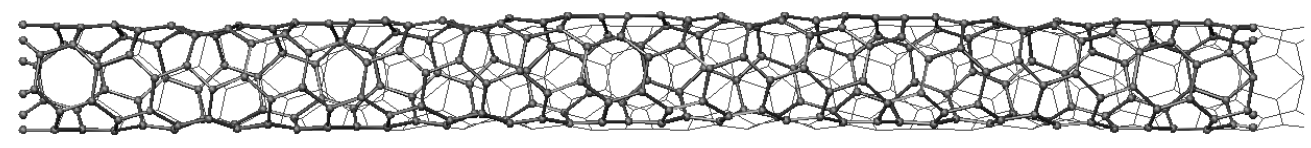

(c) $\mathrm{CH}(4,2)$ AMBER

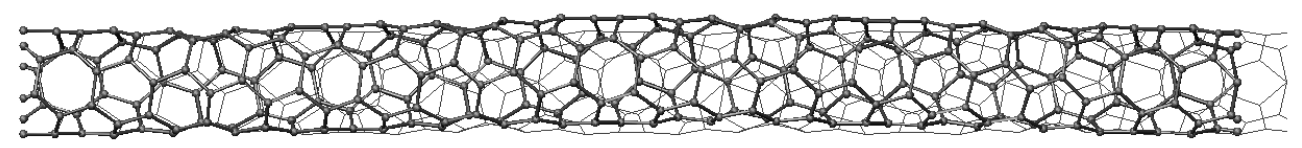

(d) $\mathrm{CH}(4,2)$ Morse

Figura 5.6: Deformadas finales SWNTs a compresión

Comentario 5.4.2 En la figura 5.5(f) se producen ciertas oscilaciones singulares en la trayectoria de equilibrio. La explicación puede residir el acoplamiento axil-flexión-torsión que poseen los nanotubos $\mathrm{CH}$ y cuyo efecto varía de unas quiralidades a otras. En concreto, al comprimir un nanotubo $\mathrm{CH}(5,3)$, la torsión y la flexión secundaria acopladas provocan un fenómeno de inversión de forma en la geometría, que degenera en oscilaciones no deseadas de la trayectoria.

Por otra parte, las deformaciones críticas en cada caso se calculan extrayendo los correspondientes factores de carga de las trayectorias de equilibrio y evaluando:

$$
\varepsilon_{c r}=\frac{\lambda_{c r}}{L}
$$

donde $\mathrm{L}$ es la longitud inicial del nanotubo. De esta forma, podemos resumir las deformaciones críticas a compresión en la tabla 5.2

\begin{tabular}{lccccc}
\cline { 2 - 6 } & \multicolumn{3}{c}{ AMBER } & \multicolumn{2}{c}{ MORSE } \\
\cline { 2 - 6 } & $\mathrm{L}(\mathrm{nm})$ & $\lambda_{c r}(\mathrm{~nm})$ & $\varepsilon_{c r}(\%)$ & $\lambda_{c r}(\mathrm{~nm})$ & $\varepsilon_{c r}(\%)$ \\
\hline $\mathrm{AC}(3,3)$ & 4.919 & 0.146 & 2.958 & 0.147 & 2.978 \\
$\mathrm{AC}(4,4)$ & 6.641 & 0.191 & 2.876 & 0.193 & 2.902 \\
\hline $\mathrm{ZZ}(5,0)$ & 4.757 & 0.133 & 2.796 & 0.132 & 2.783 \\
$\mathrm{ZZ}(7,0)$ & 6.674 & 0.189 & 2.836 & 0.190 & 2.844 \\
\hline $\mathrm{CH}(4,2)$ & 5.072 & 0.141 & 2.780 & 0.141 & 2.782 \\
$\mathrm{CH}(5,3)$ & 6.859 & 0.187 & 2.727 & 0.188 & 2.737 \\
\hline
\end{tabular}

Tabla 5.2: Deformaciones críticas a compresión 
En concordancia con [11], se observa cierta dispersión en las deformaciones críticas finales dependiendo del tamaño de incremento $l_{0} \mathrm{y}$ del número de incrementos $N I$ adoptados. Aunque la influencia de los datos de entrada en $\varepsilon_{c r}(\%)$ no se ha analizado estadísticamente de forma explícita, los valores adoptados en las simulaciones finales se añaden en la tabla 5.3 como referencia de su orden de magnitud.

\begin{tabular}{ccccc}
\cline { 2 - 5 } & \multicolumn{2}{c}{ AMBER } & \multicolumn{2}{c}{ MORSE } \\
\cline { 2 - 5 } & $l_{0}$ & $N I$ & $l_{0}$ & $N I$ \\
\hline $\mathrm{AC}(3,3)$ & 0.05 & 65 & 0.05 & 65 \\
$\mathrm{AC}(4,4)$ & 0.1 & 30 & 0.1 & 39 \\
\hline $\mathrm{ZZ}(5,0)$ & 0.05 & 60 & 0.05 & 60 \\
$\mathrm{ZZ}(7,0)$ & 0.1 & 40 & 0.1 & 40 \\
\hline $\mathrm{CH}(4,2)$ & 0.05 & 75 & 0.05 & 75 \\
$\mathrm{CH}(5,3)$ & 0.02 & 200 & 0.05 & 74 \\
\hline
\end{tabular}

Tabla 5.3: Datos de entrada en simulaciones a compresión

Comparando valores en la tabla 5.2 se puede comprobar que las deformaciones críticas son prácticamente independientes de la elección del potencial interatómico. Por tanto, podemos concluir que la inestabilidad crítica se desencadena antes de que la no linealidad constitutiva tenga lugar, de acuerdo con [27], [43], [94] у [110].

Como se puede verificar, nuestros resultados coinciden cualitativamente con otros estudios atómicos, principalmente con simulaciones MD. A saber, [115] obtiene una deformación crítica a compresión del 5\% para un SWNT de diámetro $d=1 \mathrm{~nm}$, [94] obtiene $\varepsilon_{c r}=8 \%$ y [91] estiman $\varepsilon_{c r}=$ $7.5 \%$ para el mismo diámetro. Asimismo, [121, tabla 4] y [21] predicen para nanotubos AC las deformaciones críticas resumidas en la tabla 5.4, las cuales coinciden razonablemente con los valores de la tabla 5.2

\begin{tabular}{ccclcc}
\hline \multicolumn{3}{c}{ Zhang et al. [121] } & \multicolumn{3}{c}{ Cornwell and Wille [21] } \\
\hline & $\mathrm{d}(\mathrm{nm})$ & $\varepsilon_{c r}(\%)$ & & $\mathrm{d}(\mathrm{nm})$ & $\varepsilon_{c r}(\%)$ \\
\hline $\mathrm{AC}(4,4)$ & 0.542 & 6.570 & $\mathrm{AC}(9,9)$ & 1.250 & 5.200 \\
$\mathrm{AC}(5,5)$ & 0.678 & 7.764 & $\mathrm{AC}(12,12)$ & 1.664 & 4.400 \\
$\mathrm{AC}(6,6)$ & 0.814 & 7.068 & $\mathrm{AC}(19,19)$ & 2.632 & 3.200 \\
$\mathrm{AC}(7,7)$ & 0.949 & 6.271 & & & \\
\hline
\end{tabular}

Tabla 5.4: Deformaciones críticas de compresión con simulaciones MD

También, [110, fig. 2] obtiene casi los mismos valores que [21] para el mismo rango de diámetros. Dentro del marco de la Mecánica Molecular, [11] estiman mediante el código comercial Materials Studio ${ }^{\circledR}$ una deformación crítica media $\bar{\varepsilon}_{c r}=6.6 \%$ para un $\mathrm{ZZ}(9,0)$ de $5.3 \mathrm{~nm}$ de longitud y una $\bar{\varepsilon}_{c r}=7.6 \%$ para un $\mathrm{AC}(5,5)$ de $4.7 \mathrm{~nm}$ de longitud. Además, [16] llegan a $\varepsilon_{c r}=10.7 \%$ para un $\mathrm{AC}(7,7)$ y $\varepsilon_{c r}=6.9 \%$ para un nanotubo $\mathrm{AC}(10,10)$ con una técnica basada en MM que minimiza la energía potencial total. Todos estos valores están en el rango de los resultados presentados en la tabla 5.2 mediante el modelo MSM.

Una cuestión especialmente controvertida que ha sido tratada ampliamente en varias referencias [11], [16], [79], [115], es la aplicabilidad de los modelos continuos en la predicción de deformaciones críticas de compresión de SWNTs. De acuerdo con [11], la presencia de imperfecciones geométricas en la estructura atómica (que no se pueden plasmar explícitamente en los modelos continuos) afecta a las deformaciones críticas de inestabilidad de SWNTs. Así, la aplicabilidad del continuo resulta dudosa en el análisis no lineal geométrico de nanotubos. Incluso si se defiende la aplicabilidad de modelos tipo viga o lámina [16] validados con técnicas atómicas, se acentúa la precaución necesaria 
al definir ciertos parámetros mecánicos.

No obstante, teniendo en cuenta las esbelteces de los SWNTs simulados a compresión de la tabla 5.1 y las geometrías deformadas de la figura 5.6. se produce un modo de inestabilidad de pandeo tipo viga. Como curiosidad, podemos comparar las deformaciones críticas obtenidas en esta tesis con las obtenidas en [115, ec. (2)] para modos de fallo tipo viga (ver tabla 5.5], alcanzando un sesgo máximo relativo del $20 \%$.

\begin{tabular}{lccc}
\cline { 2 - 4 } & AMBER & MORSE & {$[115]$} \\
\cline { 2 - 4 } & $\varepsilon_{c r}(\%)$ & $\varepsilon_{c r}(\%)$ & $\varepsilon_{c r}(\%)$ \\
\hline $\mathrm{AC}(3,3)$ & 2.958 & 2.978 & 3.375 \\
$\mathrm{AC}(4,4)$ & 2.876 & 2.902 & 3.292 \\
\hline $\mathrm{ZZ}(5,0)$ & 2.796 & 2.783 & 3.341 \\
$\mathrm{ZZ}(7,0)$ & 2.836 & 2.844 & 3.327 \\
\hline $\mathrm{CH}(4,2)$ & 2.780 & 2.782 & 3.293 \\
$\mathrm{CH}(5,3)$ & 2.727 & 2.737 & 3.150 \\
\hline
\end{tabular}

Tabla 5.5: Comparación con Yakobson et al. [115, eq. (2)] a compresión

\subsubsection{Comportamiento a flexión}

Los nanotubos sometidos a cargas flectoras se resumen en la tabla 5.6. Se han escogido nanotubos más cortos que los sometidos a compresión porque esta sección está enfocada al análisis del modo de abolladura más que al modo de pandeo (admitiendo esta terminología propia del cálculo de estructuras). Una descripción esquemática de las condiciones de sustentación y de los desplazamientos impuestos para el comportamiento de flexión viene representada en la figura 5.7.

\begin{tabular}{lccc}
\hline Quiralidad & $\mathrm{d}(\mathrm{nm})$ & $\mathrm{L}(\mathrm{nm})$ & $\mathrm{L} / \mathrm{d}$ \\
\hline $\mathrm{AC}(3,3)$ & 0.407 & 1.230 & 3.02 \\
$\mathrm{AC}(4,4)$ & 0.542 & 1.722 & 3.17 \\
\hline $\mathrm{ZZ}(5,0)$ & 0.391 & 1.349 & 3.45 \\
$\mathrm{ZZ}(7,0)$ & 0.548 & 1.775 & 3.24 \\
\hline $\mathrm{CH}(4,2)$ & 0.414 & 1.240 & 2.99 \\
$\mathrm{CH}(5,3)$ & 0.548 & 1.789 & 3.26 \\
\hline
\end{tabular}

Tabla 5.6: SWNTs ensayados a flexión

Las posibilidades de definición de los desplazamientos impuestos $\Delta \overline{\mathbf{u}}$ en el extremo derecho del nanotubo pueden clasificarse en dos grandes grupos:

1. El extremo derecho del eje del cilindro describe una curva definida en el plano.

2. El extremo derecho traza una trayectoria tal que las directrices consecutivas en el proceso de carga constituyen una familia de curvas conocidas que cumplen una propiedad definida.

Comentario 5.4.3 Aunque en algunas referencias consultadas que tratan el problema de la flexión de SWNTs, ya sea mediante técnicas MD [115] o mediante Elementos Finitos [108], se imponen desplazamientos impuestos en ambos extremos del SWNT, aquí se ha preferido mantener un extremo fijo con el objetivo de evitar el mal condicionamiento numérico de la matriz de rigidez tangente por la aparición de posibles mecanismos no deseados. 


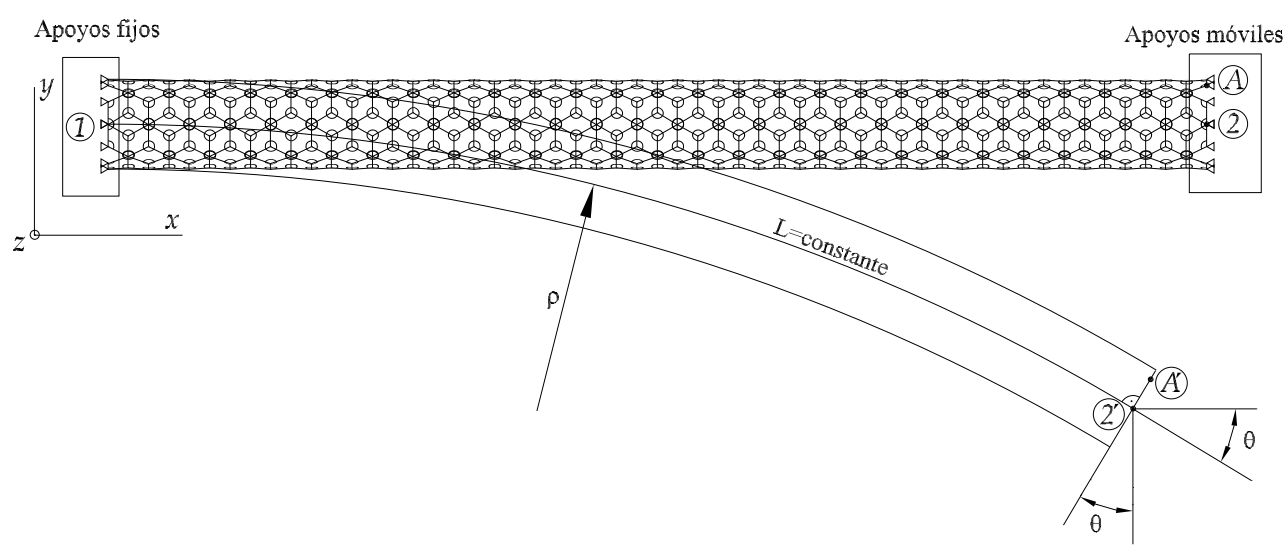

Figura 5.7: Esquema de sustentación a flexión

En nuestro caso se adopta la opción 2, donde se supone que la directriz en una configuración genérica de equilibrio es un arco de circunferencia de longitud constante. Además, la tangente inicial del arco de circunferencia será el eje del nanotubo en la configuración inicial y los apoyos móviles en el extremo derecho verificarán un movimiento de sólido rígido que mantiene la sección transversal ortogonal a la directriz deformada a lo largo del proceso de carga. De esta forma, se verifica la siguiente relación para cada configuración de equilibrio:

$$
\rho \theta=L
$$

Adoptando el mismo sistema de referencia definido en la sección 5.4.1 y tomando el factor de carga para la respuesta a flexión como $\lambda=\theta$, los desplazamientos del punto central del extremo derecho se pueden expresar para el primer incremento como:

$$
\begin{aligned}
& \Delta \bar{u}_{1}^{2}=-\left(L-\rho \sin \Delta \lambda_{1}\right)=-L\left(1-\frac{\sin \Delta \lambda_{1}}{\Delta \lambda_{1}}\right) \\
& \Delta \bar{v}_{1}^{2}=-\rho\left(1-\cos \Delta \lambda_{1}\right)=-\frac{L}{\Delta \lambda_{1}}\left(1-\cos \Delta \lambda_{1}\right) \\
& \Delta \bar{w}_{1}^{2}=0
\end{aligned}
$$

Tomando ahora un apoyo genérico (nodo A) del extremo derecho del nanotubo, sus desplazamientos impuestos se escriben:

$$
\begin{aligned}
& \Delta \bar{u}_{1}^{A}=\Delta \bar{u}_{1}^{2}+y_{A} \sin \Delta \lambda_{1}=-L\left(1-\frac{\sin \Delta \lambda_{1}}{\Delta \lambda_{1}}\right)+y_{A} \sin \Delta \lambda_{1} \\
& \Delta \bar{v}_{1}^{A}=\Delta \bar{v}_{1}^{2}+y_{A} \cos \Delta \lambda_{1}=-\left(\frac{L}{\Delta \lambda_{1}}+y_{A}\right)\left(1-\cos \Delta \lambda_{1}\right) \\
& \Delta \bar{w}_{1}^{A}=\Delta \bar{w}_{1}^{2}
\end{aligned}
$$

Si suponemos que el tamaño de incremento nos permite considerar $\Delta \lambda_{n}$ suficientemente pequeño, las ecuaciones (5.77) se pueden simplificar según:

$$
\begin{gathered}
\left\{\begin{array}{c}
\Delta \bar{u}_{1}^{A} \\
\Delta \bar{v}_{1}^{A} \\
\Delta \bar{w}_{1}^{A}
\end{array}\right\}=\Delta \lambda_{1}\left\{\begin{array}{c}
y_{A} \\
-L / 2 \\
0
\end{array}\right\} \\
\Delta \overline{\mathbf{u}}_{1}^{A}=\Delta \lambda_{1} \widetilde{\mathbf{u}}_{1}^{A}
\end{gathered}
$$


Para los siguientes incrementos empleamos las ecuaciones (5.76) al principio y al final de cada uno. Así, restando las expresiones resultantes se llega a:

$$
\begin{aligned}
\Delta \bar{u}_{n}^{2} & =\bar{u}_{n+1}^{2}-\bar{u}_{n}^{2}=L\left[\frac{\sin \lambda_{n+1}}{\lambda_{n+1}}-\frac{\sin \lambda_{n}}{\lambda_{n}}\right] \\
\Delta \bar{v}_{n}^{2} & =\bar{v}_{n+1}^{2}-\bar{v}_{n}^{2}=-L\left[\frac{1-\cos \lambda_{n+1}}{\lambda_{n+1}}-\frac{1-\cos \lambda_{n}}{\lambda_{n}}\right] \\
\Delta \bar{w}_{n}^{2} & =\bar{w}_{n+1}^{2}-\bar{w}_{n}^{2}=0
\end{aligned}
$$

Análogamente, podemos añadir los desplazamientos incrementales del nudo A a la ec. 5.79, consiguiendo:

$$
\begin{aligned}
\Delta \bar{u}_{n}^{A} & =\Delta \bar{u}_{n}^{2}+y_{A}\left(\sin \lambda_{n+1}-\sin \lambda_{n}\right) \\
\Delta \bar{v}_{n}^{A} & =\Delta \bar{v}_{n}^{2}+y_{A}\left(\cos \lambda_{n+1}-\cos \lambda_{n}\right) \\
\Delta \bar{w}_{n}^{A} & =\Delta \bar{w}_{n}^{2}
\end{aligned}
$$

Tras algunas manipulaciones algebraicas y suponiendo que $\Delta \lambda_{n}$ es suficientemente pequeño, se obtienen las siguientes expresiones simplificadas para los desplazamientos incrementales en el nudo A:

$$
\begin{gathered}
\left\{\begin{array}{c}
\Delta \bar{u}_{n}^{A} \\
\Delta \bar{v}_{n}^{A} \\
\Delta \bar{w}_{n}^{A}
\end{array}\right\}=\Delta \lambda_{n}\left\{\begin{array}{c}
\frac{L}{\lambda_{n}}\left(\cos \lambda_{n}-\frac{\sin \lambda_{n}}{\lambda_{n}}\right)+y_{A} \cos \lambda_{n} \\
\frac{L}{\lambda_{n}}\left(\frac{1-\cos \lambda_{n}}{\lambda_{n}}-\sin \lambda_{n}\right)-y_{A} \sin \lambda_{n} \\
0
\end{array}\right\} \\
\Delta \overline{\mathbf{u}}_{n}^{A}=\Delta \lambda_{n} \widetilde{\mathbf{u}}_{n}^{A}
\end{gathered}
$$

Como se ha mencionado en la sección 5.3.2, los desplazamientos impuestos se actualizan exclusivamente en la fase predictiva llevando a la necesaria redistribución de esfuerzos en la correctiva. De ahí que el superíndice $\mathrm{k}$ desaparezca de las ecuaciones (5.76) a la (5.81).

Las trayectorias de equilibrio no lineales obtenidas a flexión se representan en la figura 5.8, donde se ha adoptado como desplazamiento representativo $u_{\rho}$ el de un nudo situado en torno al punto medio de la zona comprimida (inferior) del nanotubo.

Adicionalmente, se proporcionan algunas geometrías deformadas al final del último incremento en las figuras 5.9, como ejemplo del modo de inestabilidad local o abolladura obtenido a flexión.

Las imperfecciones iniciales en simulaciones de flexión se han adoptado como una carga puntual en la dirección del eje $\{y\}$ en un nudo cercano al punto medio de la zona comprimida (inferior) del SWNT, tanteando sus valores hasta obtener la respuesta porstcrítica más prolongada.

La deformación crítica adoptada para describir la inestabilidad a flexión es la curvatura crítica, calculada a partir de cada factor de carga crítico (rotación en el extremo libre) como:

$$
\kappa_{c r}=\frac{\lambda_{c r}}{L}
$$

Nótese que la ecuación (5.82) es una aproximación a la curvatura real de abolladura, ya que la rotación en el extremo libre $\lambda=\theta$ no es necesariamente igual a la rotación en la sección transversal donde se produce la inestabilidad. En efecto, aunque la directriz en una configuración genérica se ha supuesto como un arco de circunferencia con curvatura constante, la redistribución de esfuerzos en la fase correctiva de cada incremento introduce una desviación acumulativa de dicha curva.

Extrayendo de cada trayectoria de equilibrio $\lambda_{c r}$ y empleando (5.82), podemos resumir las curvaturas críticas obtenidas (en $\mathrm{nm}^{-1}$ ) en la tabla 5.7. Además, los datos de entrada para simulaciones de flexión (tal como se hizo a compresión) se incluyen en la tabla 5.8 como orientación respecto a su orden de magnitud. 


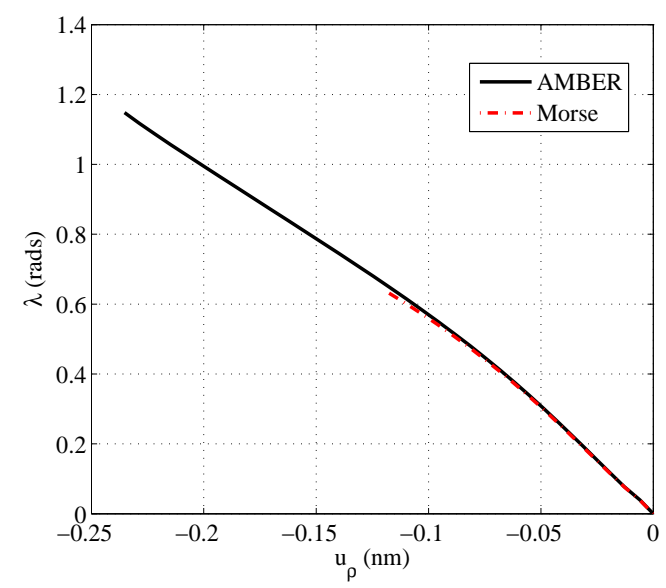

(a) $\mathrm{ZZ}(5,0)$ flexión

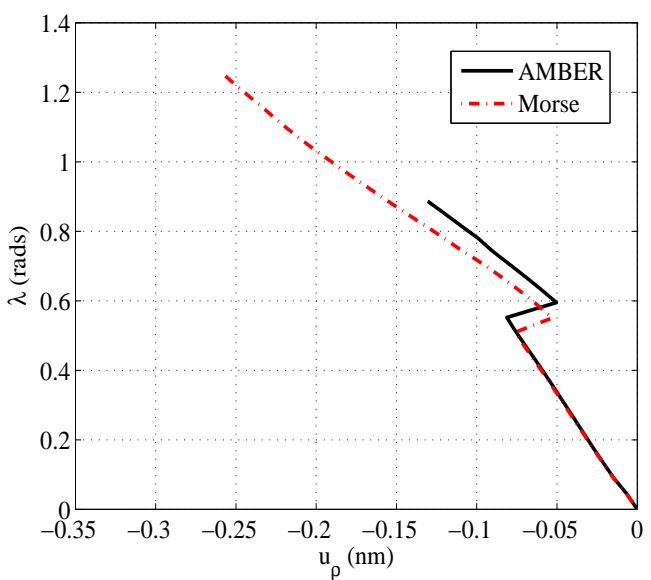

(c) $\mathrm{AC}(3,3)$ flexión

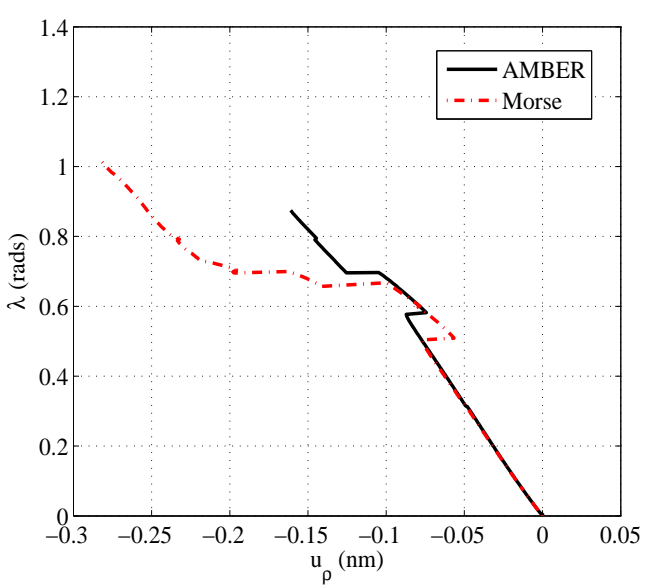

(e) $\mathrm{CH}(4,2)$ flexión

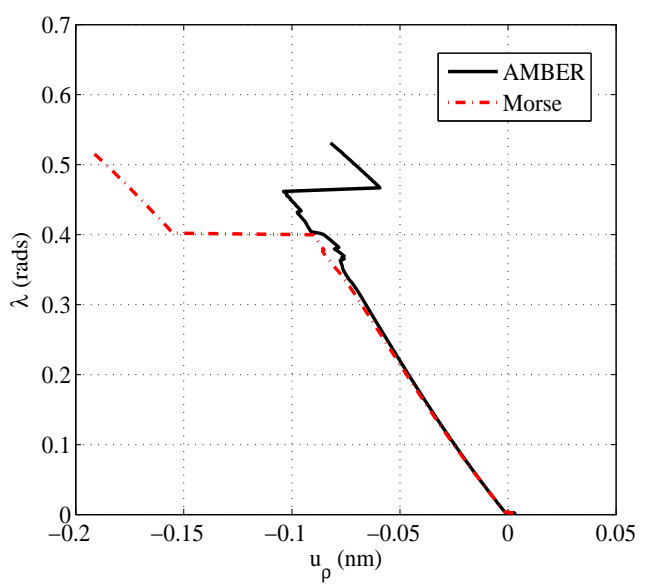

(b) $\mathrm{ZZ}(7,0)$ flexión

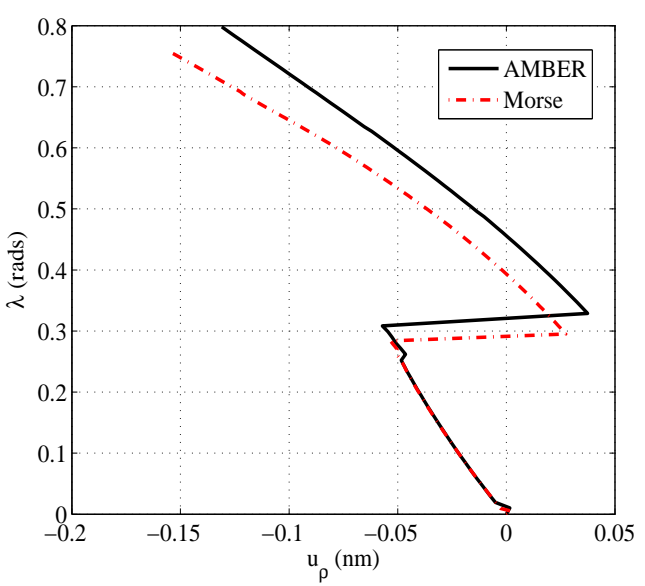

(d) $\mathrm{AC}(4,4)$ flexión

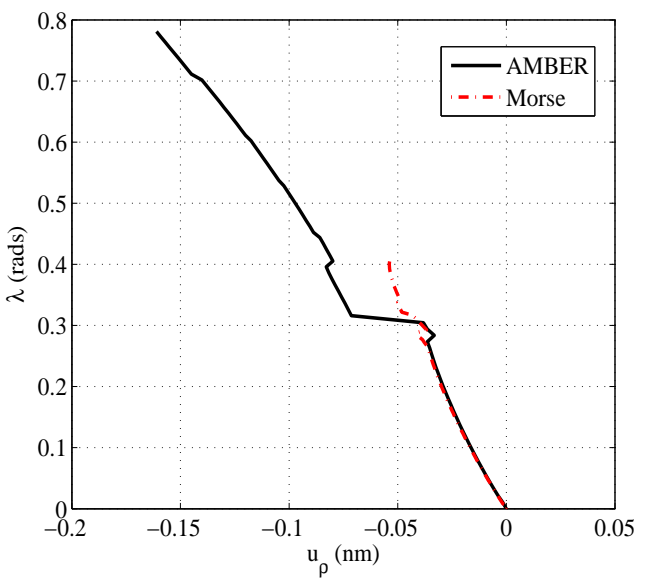

(f) $\mathrm{CH}(5,3)$ flexión

Figura 5.8: Trayectorias no lineales SWNTs flexión 


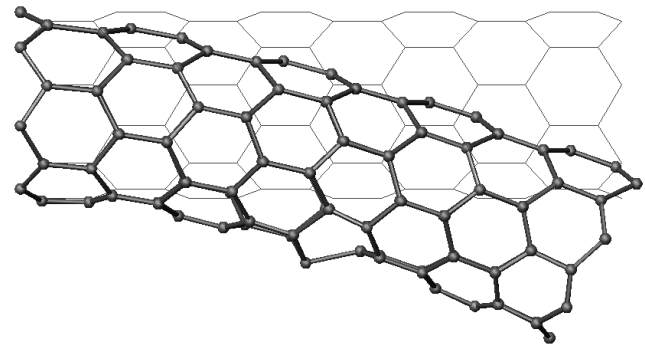

(a) $\mathrm{ZZ}(7,0)$ Morse

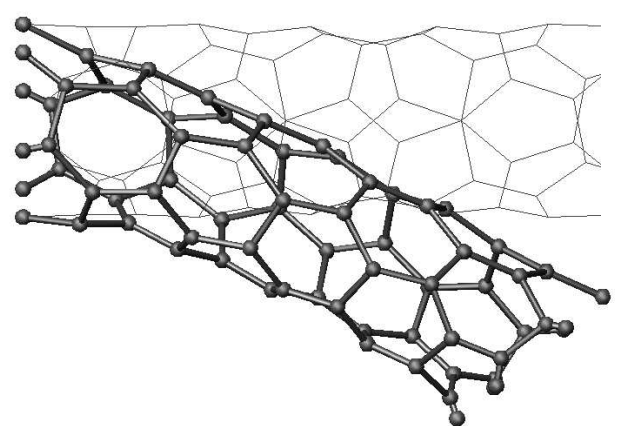

(c) $\mathrm{CH}(4,2) \mathrm{AMBER}$

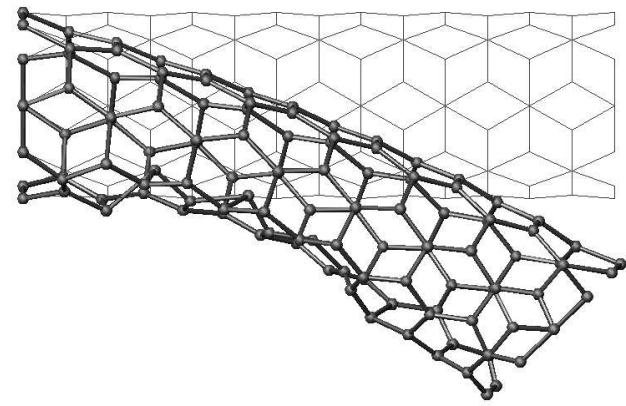

(b) $\mathrm{AC}(4,4) \mathrm{AMBER}$

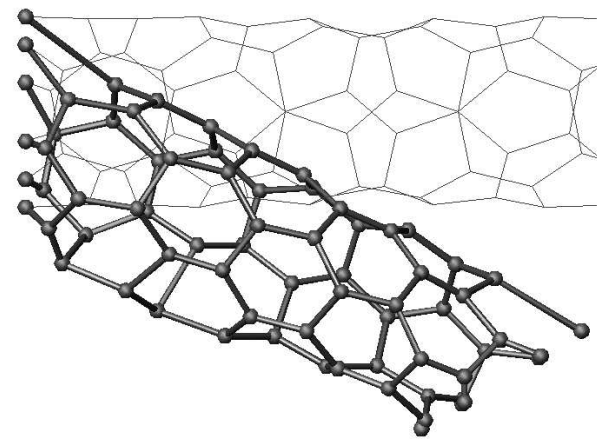

(d) $\mathrm{CH}(4,2)$ Morse

Figura 5.9: Deformadas finales $S W N T$ s a flexión

\begin{tabular}{lccccc}
\cline { 2 - 6 } & \multicolumn{3}{c}{ AMBER } & \multicolumn{2}{c}{ MORSE } \\
\cline { 2 - 6 } & $\mathrm{L}(\mathrm{nm})$ & $\lambda_{c r}(\mathrm{rad})$ & $\kappa_{c r}\left(\mathrm{~nm}^{-1}\right)$ & $\lambda_{c r}(\mathrm{rad})$ & $\kappa_{c r}\left(\mathrm{~nm}^{-1}\right)$ \\
\hline $\mathrm{AC}(3,3)$ & 1.230 & 0.552 & 0.449 & 0.510 & 0.414 \\
$\mathrm{AC}(4,4)$ & 1.722 & 0.308 & 0.179 & 0.284 & 0.165 \\
\hline $\mathrm{ZZ}(5,0)$ & 1.349 & 1.148 & 0.851 & 0.636 & 0.472 \\
$\mathrm{ZZ}(7,0)$ & 1.775 & 0.462 & 0.260 & 0.400 & 0.225 \\
\hline $\mathrm{CH}(4,2)$ & 1.240 & 0.577 & 0.466 & 0.504 & 0.407 \\
$\mathrm{CH}(5,3)$ & 1.789 & 0.304 & 0.170 & 0.293 & 0.164 \\
\hline
\end{tabular}

Tabla 5.7: Curvaturas críticas de abolladura a flexión

Comentario 5.4.4 Los valores $\lambda_{c r}$ extraídos de las trayectorias de equilibrio corresponden a los valores donde se produce la primera discontinuidad significativa en cada curva. Sin embargo, en las trayectorias donde dicha discontinuidad no puede observarse (por ej, ver fig. 5.8(a) el parámetro de carga crítico se ha adoptado como el valor final de la curva, ya que en ese punto se produjo la divergencia normalmente asociada a la inestabilidad propia de un punto de bifurcación.

Comparando valores de la tabla 5.7 podemos deducir que las curvaturas críticas de abolladura obtenidas con cada potencial interatómico son diferentes. Sin embargo, no se observa una tendencia sistemática clara, ya que las diferencias constitutivas debidas a la función potencial aparecen acopladas con varios efectos, como la dispersión producida por los datos de entrada o los efectos de borde. Éstos últimos serán más notables en simulaciones de nanotubos cortos, como los adoptados para flexión en nuestro caso. 


\begin{tabular}{ccccc}
\cline { 2 - 5 } & \multicolumn{2}{c}{ AMBER } & \multicolumn{2}{c}{ MORSE } \\
\cline { 2 - 5 } & $l_{0}$ & $N I$ & $l_{0}$ & $N I$ \\
\hline $\mathrm{AC}(3,3)$ & 0.1 & 22 & 0.1 & 35 \\
$\mathrm{AC}(4,4)$ & 0.04 & 90 & 0.02 & 180 \\
\hline $\mathrm{ZZ}(5,0)$ & 0.1 & 34 & 0.1 & 19 \\
$\mathrm{ZZ}(7,0)$ & 0.01 & 250 & 0.01 & 250 \\
\hline $\mathrm{CH}(4,2)$ & 0.01 & 240 & 0.01 & 327 \\
$\mathrm{CH}(5,3)$ & 0.05 & 80 & 0.02 & 100
\end{tabular}

Tabla 5.8: Datos de entrada en simulaciones a flexión

Una rápida inspección a las geometrías deformadas de las figuras 5.9 y 5.10 permite advertir cierto aplanamiento de la sección transversal del nanotubo en la zona inferior, como ha sido descrito en [43], [105], [115]. Este fenómeno, denominado efecto Brazier [8] en mecánica del continuo, aparece principalmente en láminas cilíndricas aunque el presente modelo MSM lo reproduce de forma similar en SWNTs. Pese a esto, en algunos casos (ver fig. 5.9(d)p la geometría deformada final se ve distorsionada por los efectos de borde, los cuales amplifican la inestabilidad cerca del extremo izquierdo del nanotubo debido a la existencia de algunos enlaces fuertemente comprimidos. Asimismo, la aparición de escalones críticos secundarios (ver fig.5.8(e) asociados a otros modos de abolladura queda plasmado en estas geometrías deformadas inesperadas.

En general, nuestros resultados coinciden razonablemente con otras referencias publicadas. Por ejemplo, [81] propone una rotación crítica de abolladura de $\theta_{c r}=1.1 \mathrm{rad}$ para un nanotubo ZZ $(13,0)$ de $2 \mathrm{~nm}$ de diámetro y $8 \mathrm{~nm}$ de longitud mediante una modelización de elementos finitos (FEM) validada con técnicas MD. Estos valores se traducen en una curvatura crítica de $\kappa_{c r}=0.1375 \mathrm{~nm}^{-1}$, ligeramente inferior que nuestros resultados para un diámetro mayor. En esta línea, [43] utiliza cálculos MD para un SWNT de $1.2 \mathrm{~nm}$ de diámetro obteniendo una rotación crítica de $\theta_{c r}=30^{\circ}=0.5236 \mathrm{rad}$, más cerca del orden de magnitud de nuestros valores. Incluso en [43, ec. (2)] se establece un ajuste numérico de sus propios resultados de $\theta_{c r}$ a flexión, aunque al estar limitado al intervalo de diámetros $[1,1,5] \mathrm{nm}$, no tiene sentido emplearlo en nuestro caso.

A pesar de trabajar sobre un modelo lámina cilíndrica (recordemos la dudosa aplicabilidad de los modelos continuos) validado con técnicas MD, [115, ec. (4)] estima las curvaturas críticas para nuestro intervalo de diámetros en:

\begin{tabular}{lccc} 
& $\mathrm{d}(\mathrm{nm})$ & $\mathrm{L}(\mathrm{nm})$ & $\kappa_{c r}\left(\mathrm{~nm}^{-1}\right)$ \\
\hline $\mathrm{AC}(3,3)$ & 0.407 & 1.230 & 0.937 \\
$\mathrm{AC}(4,4)$ & 0.542 & 1.722 & 0.527 \\
\hline $\mathrm{ZZ}(5,0)$ & 0.391 & 1.349 & 1.012 \\
$\mathrm{ZZ}(7,0)$ & 0.548 & 1.775 & 0.516 \\
\hline $\mathrm{CH}(4,2)$ & 0.414 & 1.240 & 0.903 \\
$\mathrm{CH}(5,3)$ & 0.548 & 1.789 & 0.516 \\
\hline
\end{tabular}

Tabla 5.9: Curvaturas críticas de abolladura a flexión estimadas según [115, eq. (4)]

cuyos valores son razonablemente próximos a los presentados en esta tesis (ver tabla 5.7).

Para ilustrar cualitativamente la formación del modo de inestabilidad observado a flexión, representamos en la figura 5.10 las deformadas del AC $(4,4)$ con potencial AMBER para algunos incrementos intermedios. 


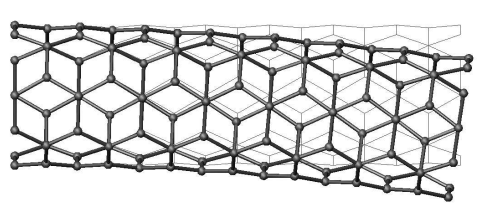

(a) $n=15$, alzado

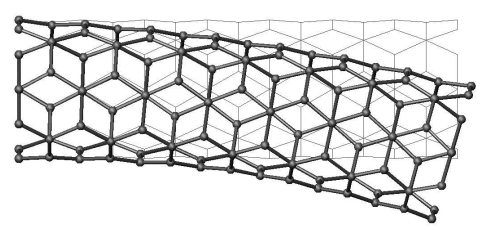

(c) $n=30$, alzado

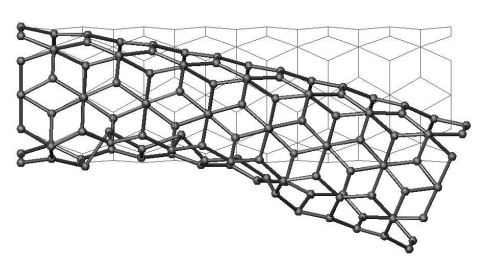

(e) $n=45$, alzado

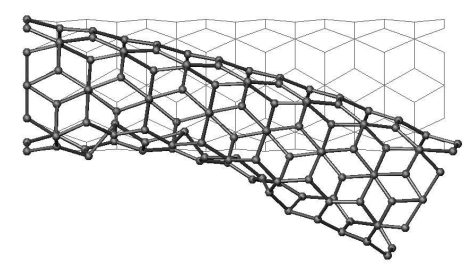

(g) $n=60$, alzado

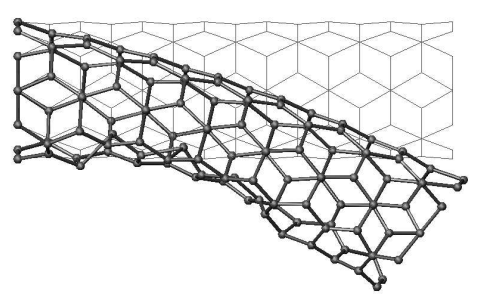

(i) $n=75$, alzado

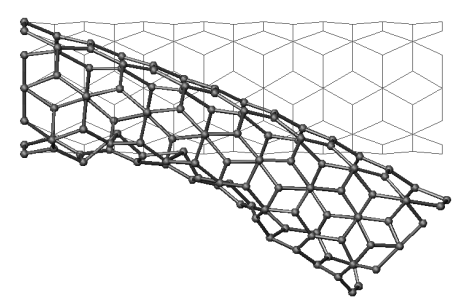

(k) $n=90$, alzado

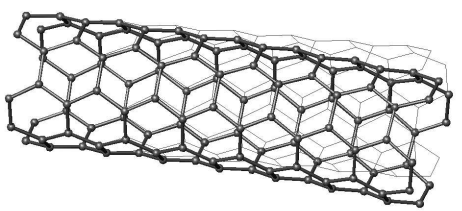

(b) $n=15$, vista $3 \mathrm{D}$

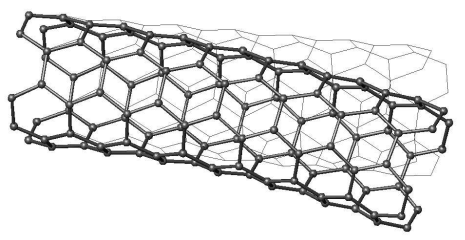

(d) $n=30$, vista $3 \mathrm{D}$

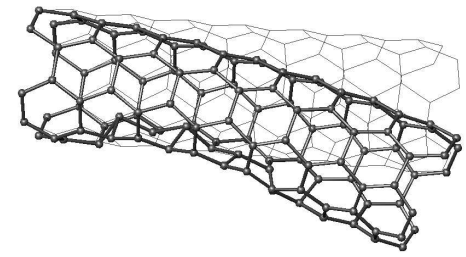

(f) $n=45$, vista $3 \mathrm{D}$

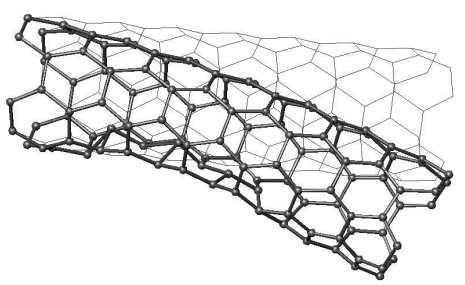

(h) $n=60$, vista $3 \mathrm{D}$

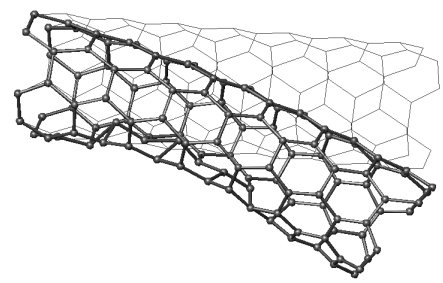

(j) $n=75$, vista $3 \mathrm{D}$

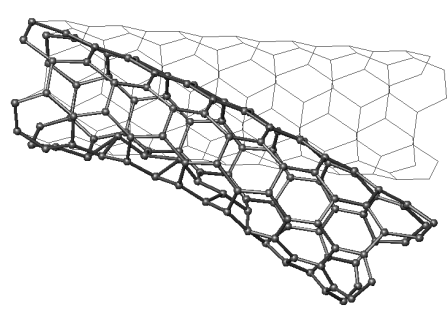

(1) $n=90$, vista 3D

Figura 5.10: Proceso de deformación AC(4,4) AMBER a flexión 


\subsubsection{Comportamiento a torsión}

Las dimensiones y quiralidades de los SWNTs simulados a torsión se resumen en la tabla 5.10 y en la figura 5.11 incluimos una descripción esquemática de las condiciones de sustentación y del sistema de desplazamientos impuestos a torsión. En este caso de carga, los apoyos móviles verifican una rotación de sólido rígido $\theta=\lambda$ en la dirección del eje $\{x\}$ positivo.

\begin{tabular}{lccc}
\hline Chirality & $\mathrm{d}(\mathrm{nm})$ & $\mathrm{L}(\mathrm{nm})$ & $\mathrm{L} / \mathrm{d}$ \\
\hline $\mathrm{AC}(3.3)$ & 0.407 & 1.476 & 3.63 \\
$\mathrm{AC}(4.4)$ & 0.542 & 1.968 & 3.63 \\
\hline $\mathrm{ZZ}(5.0)$ & 0.391 & 1.562 & 3.99 \\
$\mathrm{ZZ}(7.0)$ & 0.548 & 1.988 & 3.63 \\
\hline $\mathrm{CH}(4.2)$ & 0.414 & 1.465 & 3.54 \\
$\mathrm{CH}(5.3)$ & 0.548 & 2.028 & 3.70 \\
\hline
\end{tabular}

Tabla 5.10: $S$ WNTs ensayados a torsión

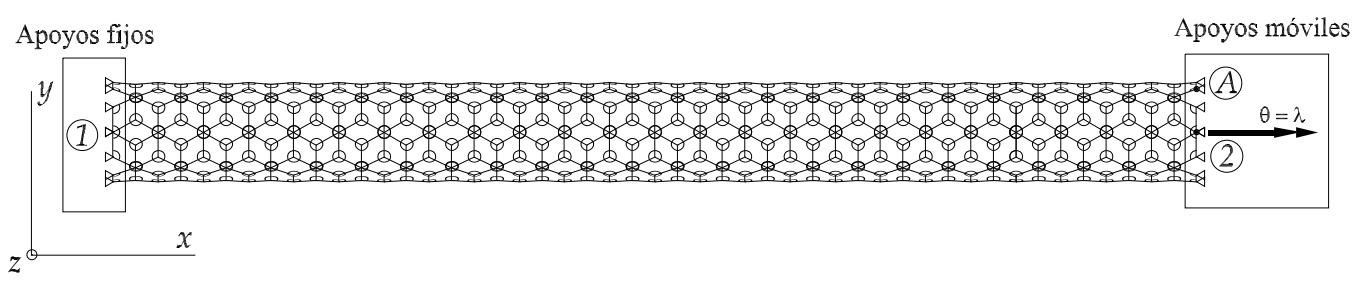

(a) Condiciones de sustentación a torsión

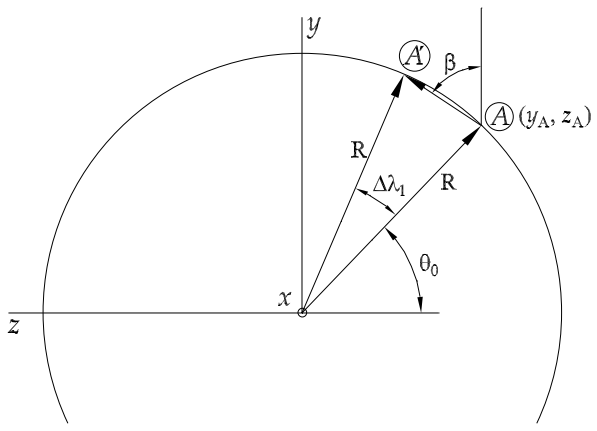

(b) Rotación extremo móvil, incremento $n=1$

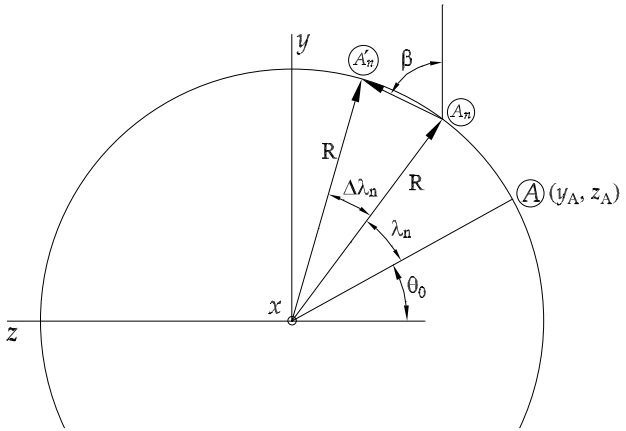

(c) Rotación extremo móvil, incremento genérico

Figura 5.11: Esquema de sustentación y desplazamientos impuestos a torsión

Orientado a la simplificación del desarrollo de las expresiones que proporcionan los desplazamientos impuestos de un apoyo genérico A en términos del parámetro de carga $\lambda$, suponemos inicialmente que los incrementos $\Delta \lambda_{n}, n=1,2, \ldots, N I$ son suficientemente pequeños. Por tanto, $\Delta \lambda_{n} \ll \lambda_{n}$ y $\Delta \lambda_{n} \ll \theta_{0}$ (ver figs. 5.11(b) and 5.11(c) . En concreto, para el primer incremento tendremos:

$$
\left|\Delta \overline{\mathbf{u}}_{1}^{A}\right| \simeq R \Delta \lambda_{1}
$$


proyectando en los ejes coordenados:

$$
\begin{aligned}
\Delta \bar{u}_{1}^{A} & =0 \\
\Delta \bar{v}_{1}^{A} & =\Delta \lambda_{1} R \cos \beta \\
\Delta \bar{w}_{1}^{A} & =\Delta \lambda_{1} R \sin \beta
\end{aligned}
$$

Puesto que $\beta \simeq \theta_{0}$, la ecuación (5.84) se convierte en:

$$
\begin{aligned}
\left\{\begin{array}{c}
\Delta \bar{u}_{1}^{A} \\
\Delta \bar{v}_{1}^{A} \\
\Delta \bar{w}_{1}^{A}
\end{array}\right\} & =\Delta \lambda_{1}\left\{\begin{array}{c}
0 \\
-z_{A} \\
y_{A}
\end{array}\right\} \\
\Delta \overline{\mathbf{u}}_{1}^{A} & =\Delta \lambda_{1} \widetilde{\mathbf{u}}_{1}^{A}
\end{aligned}
$$

Para los siguientes incrementos (fig 5.11(c) $)$, las expresiones 5.84) adoptan la forma:

$$
\begin{aligned}
\Delta \bar{u}_{n}^{A} & =0 \\
\Delta \bar{v}_{n}^{A} & =\Delta \lambda_{n} R \cos \beta \\
\Delta \bar{w}_{n}^{A} & =\Delta \lambda_{n} R \sin \beta
\end{aligned}
$$

En este caso $\beta \simeq \theta_{0}+\lambda_{n}$, luego (5.86) se puede reescribir:

$$
\begin{gathered}
\left\{\begin{array}{c}
\Delta \bar{u}_{n}^{A} \\
\Delta \bar{v}_{n}^{A} \\
\Delta \bar{w}_{n}^{A}
\end{array}\right\}=\Delta \lambda_{n}\left\{\begin{array}{c}
0 \\
-z_{A} \cos \lambda_{n}-y_{A} \sin \lambda_{n} \\
y_{A} \cos \lambda_{n}-z_{A} \sin \lambda_{n}
\end{array}\right\} \\
\Delta \overline{\mathbf{u}}_{n}^{A}=\Delta \lambda_{n} \widetilde{\mathbf{u}}_{n}^{A}
\end{gathered}
$$

Conviene recordar que los desplazamientos impuestos se actualizan exclusivamente en la fase predictiva, tal como hicimos en las secciones 5.4.1 y 5.4.2.

Comentario 5.4.5 Extendemos el razonamiento expuesto en el comentario 5.4 .3 al caso de la torsión. Es decir, se vuelve a adoptar un extremo del SWNT como fijo para evitar posibles mecanismos no deseados por mal condicionamiento numérico de la matriz de rigidez tangente.

Representamos en la figura 5.12 las trayectorias de equilibrio obtenidas a torsión, que describen la respuesta del desplazamiento transversal al eje del SWNT $u_{\rho}$, frente al parámetro de carga $\lambda=\theta$ (rotación del extremo móvil).

En este caso, las imperfecciones iniciales se introducen como dos cargas puntuales en la dirección del eje $\{y\}$ en sentidos opuestos, ya sea estirando o aplanando la sección transversal del tubo. Sus valores han sido tanteados para obtener la respuesta postcrítica a la abolladura más extensa. 


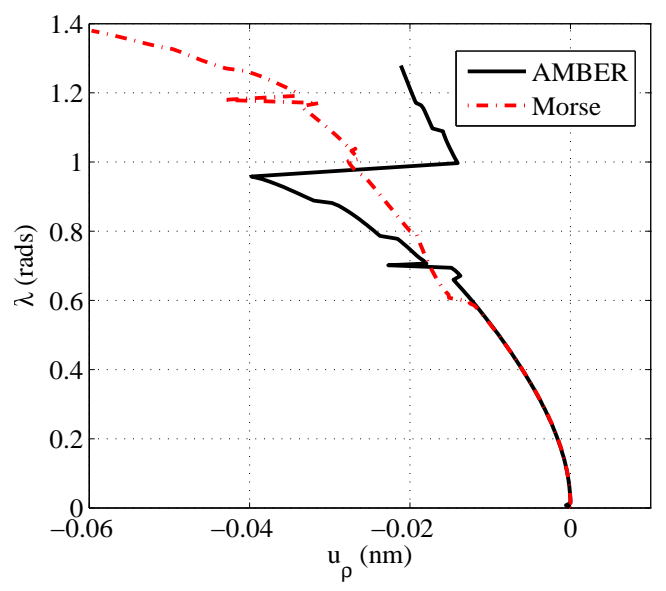

(a) $\mathrm{ZZ}(5,0)$ torsión

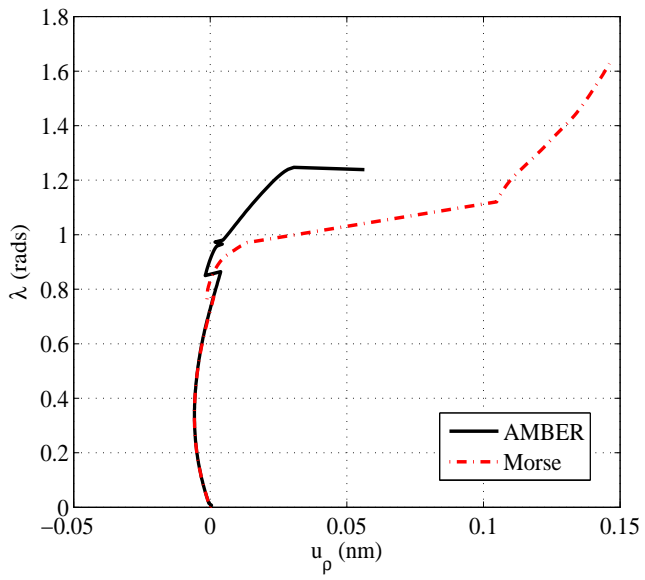

(c) $\mathrm{AC}(3,3)$ torsión

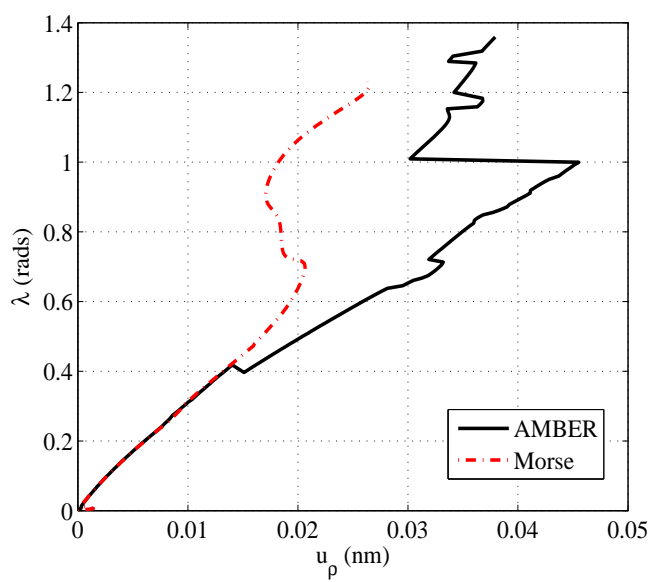

(e) $\mathrm{CH}(4,2)$ torsión

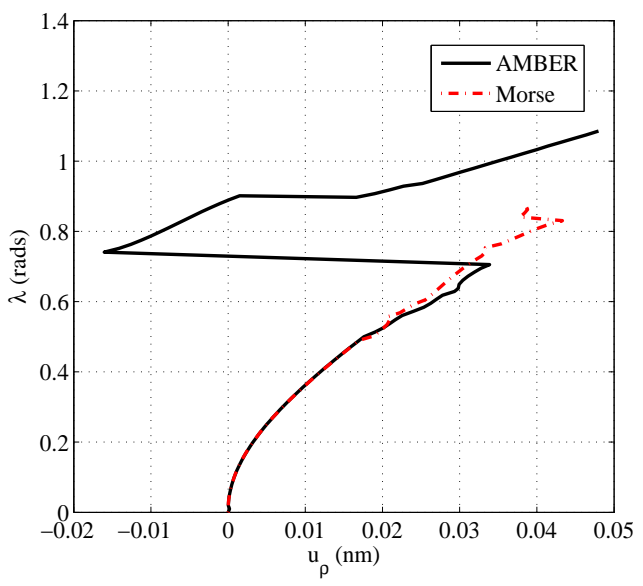

(b) $\mathrm{ZZ}(7,0)$ torsión

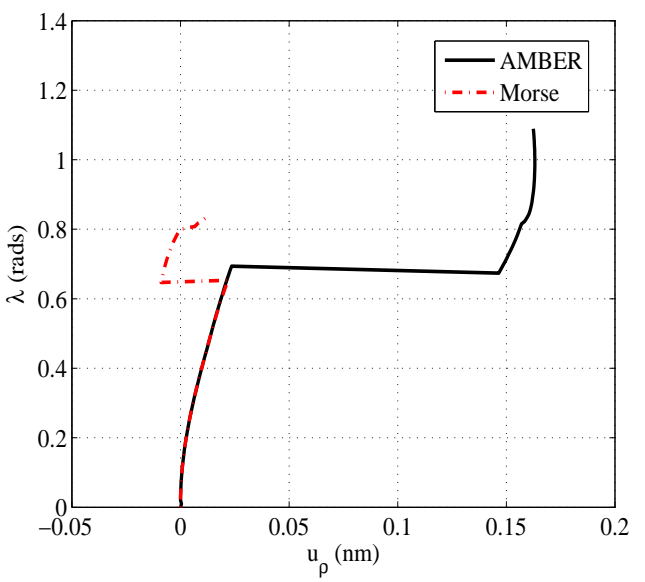

(d) $\mathrm{AC}(4,4)$ torsión

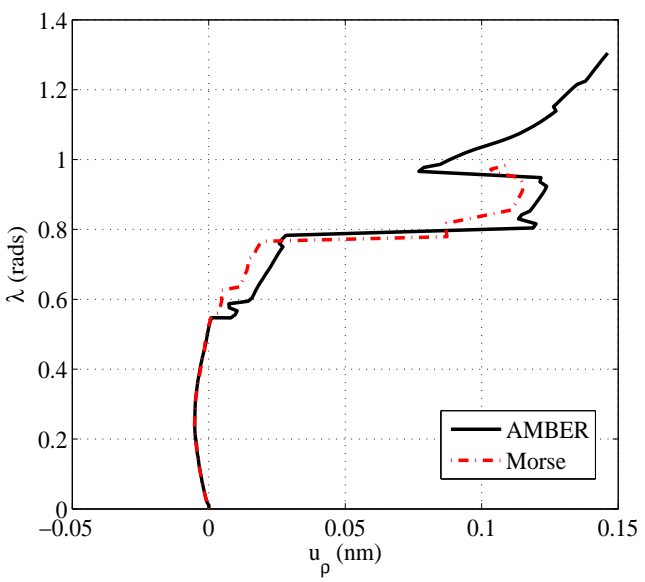

(f) $\mathrm{CH}(5,3)$ torsión

Figura 5.12: Trayectorias no lineales SWNTs torsión 
Como hicimos en compresión y flexión, incluimos los valores de entrada para simulaciones a torsión en la tabla 5.11 para dar una orientación respecto a su orden de magnitud.

\begin{tabular}{ccccc}
\cline { 2 - 5 } & \multicolumn{2}{c}{ AMBER } & \multicolumn{2}{c}{ MORSE } \\
\cline { 2 - 5 } & $l_{0}$ & $N I$ & $l_{0}$ & $N I$ \\
\hline $\mathrm{AC}(3,3)$ & 0.01 & 164 & 0.01 & 185 \\
$\mathrm{AC}(4,4)$ & 0.02 & 100 & 0.02 & 80 \\
\hline $\mathrm{ZZ}(5,0)$ & 0.01 & 160 & 0.01 & 175 \\
$\mathrm{ZZ}(7,0)$ & 0.02 & 100 & 0.02 & 80 \\
\hline $\mathrm{CH}(4,2)$ & 0.01 & 180 & 0.01 & 160 \\
$\mathrm{CH}(5,3)$ & 0.02 & 120 & 0.02 & 92 \\
\hline
\end{tabular}

Tabla 5.11: Datos de entrada en simulaciones a torsión

Comentario 5.4.6 Las trayectorias de equilibrio se ajustan sensiblemente a una función senoidal en el rango de la linealidad (ver figs. 5.12(a) y 5.12(b) . Esta idea se ve desvirtuada en algunas de las curvas representadas por el reducido tamaño de los SWNTs ensayados, que se traduce en un menor grado de simetría radial, y por los efectos de borde debidos a la necesidad de mantener circulares las dos secciones extremas.

Adicionalmente, se proporcionan algunas geometrías deformadas finales en las figuras 5.13, como ejemplo del modo de inestabilidad local obtenido a torsión. De hecho, este mecanismo de fallo se traduce en un aplanamiento de la sección transversal asociado a una geometría deformada tipo banda o hélice, como se ha determinado en algunas referencias [90, fig. 6], [109, fig. 4], [115, fig. 4].

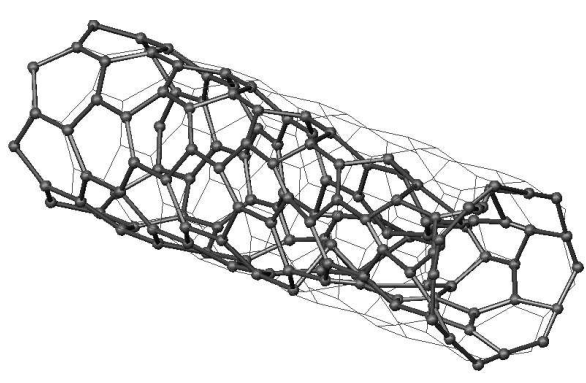

(a) $\mathrm{ZZ}(7,0)$ AMBER

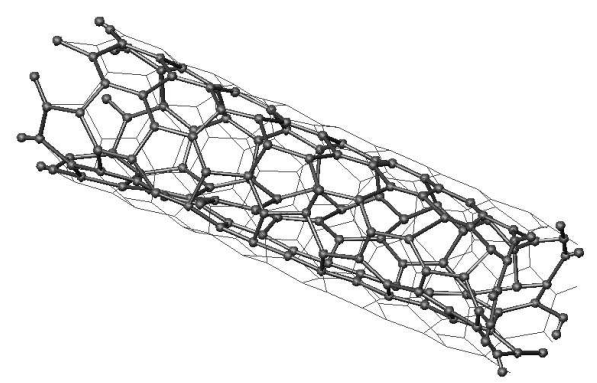

(c) $\mathrm{CH}(5,3)$ AMBER

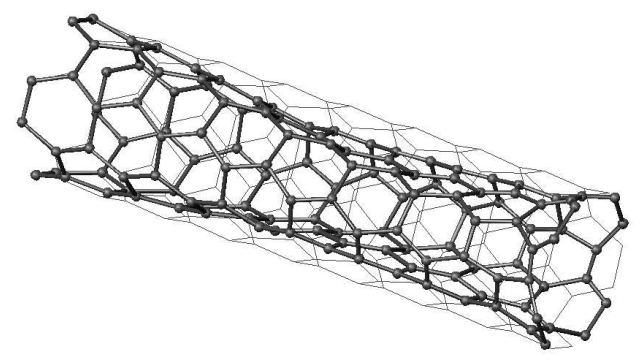

(b) $\mathrm{AC}(4,4)$ Morse

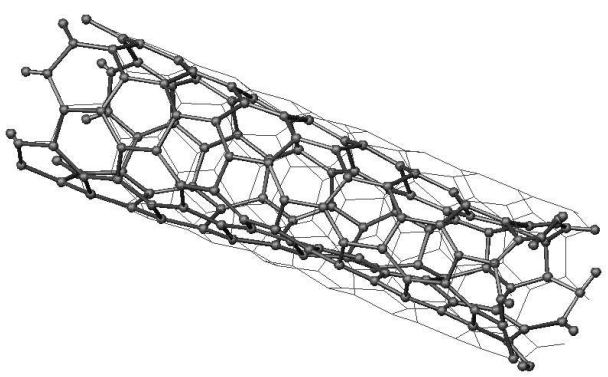

(d) $\mathrm{CH}(4,2)$ Morse

Figura 5.13: Algunas geometrías deformadas a torsión

Para ilustrar cualitativamente el desarrollo del modo de fallo a torsión (ribbon-like failure), representamos en las figuras 5.14 las deformadas del AC $(4,4)$ con potencial AMBER para algunos 
incrementos intermedios. Como puede verificarse, el modelo MSM reproduce razonablemente el fallo por aplanamiento constatado con técnicas MD [109, fig. 4].

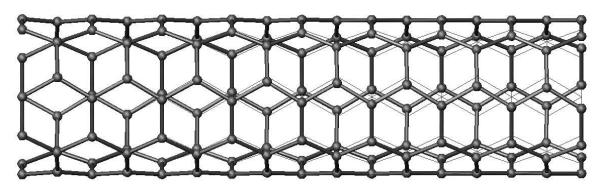

(a) $n=15$, alzado

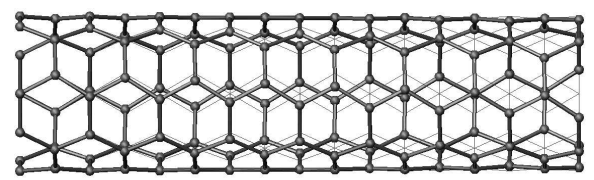

(c) $n=30$, alzado

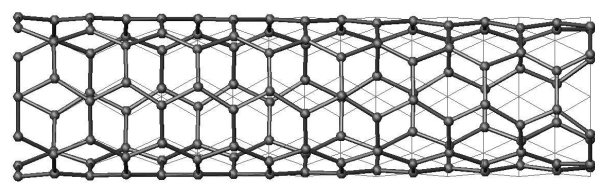

(e) $n=45$, alzado

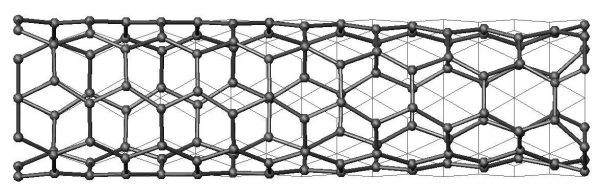

(g) $n=60$, alzado

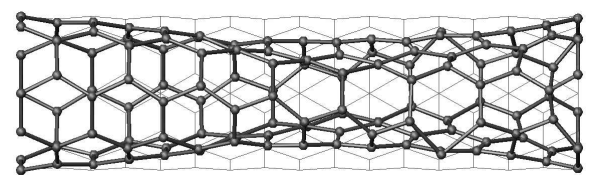

(i) $n=75$, alzado

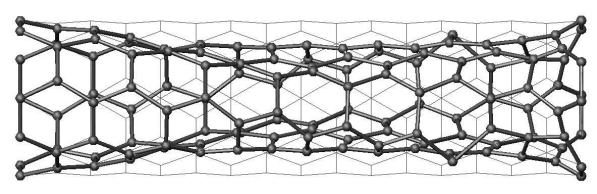

(k) $n=100$, alzado

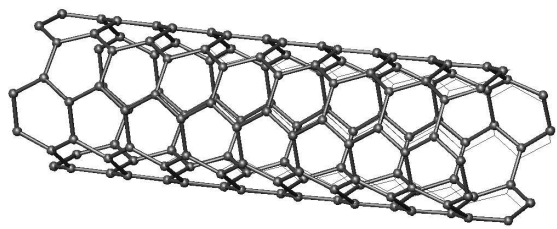

(b) $n=15$, vista 3D

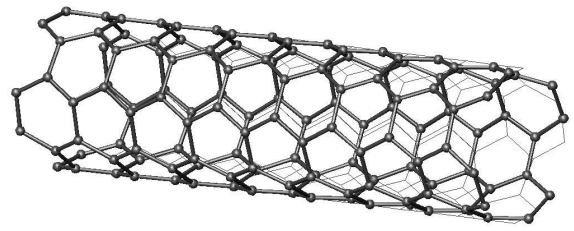

(d) $n=30$, vista 3D

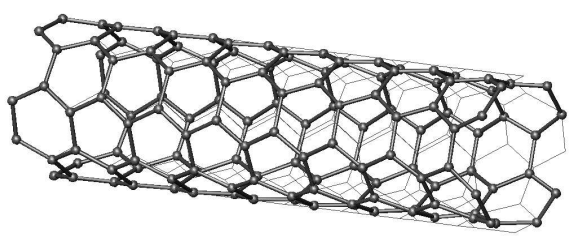

(f) $n=45$, vista $3 \mathrm{D}$

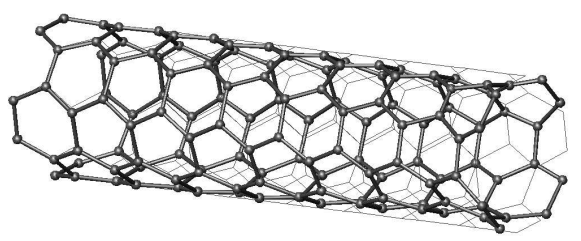

(h) $n=60$, vista $3 \mathrm{D}$

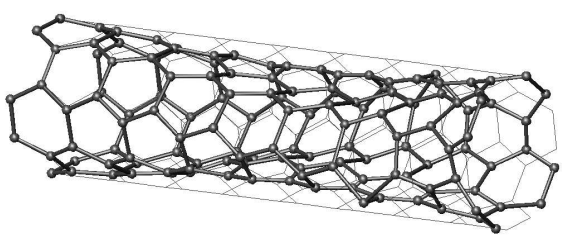

(j) $n=75$, vista 3D

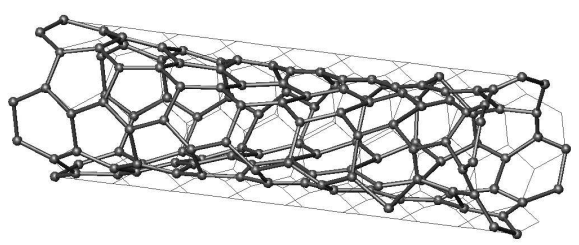

(1) $n=100$, vista $3 \mathrm{D}$

Figura 5.14: Proceso de deformación AC $(4,4) A M B E R$ a torsión 
A partir del escalón de discontinuidad de cada trayectoria de equilibrio extraemos la rotación crítica en el extremo móvil (equivalente a $\lambda_{c r}$ ), obteniendo los valores resumidos en la tabla 5.12. Extendemos lo expuesto en el comentario 5.4.5 al caso de la torsión, tomando como rotación crítica la final de la curva en los casos donde no se ha observado escalón de discontinuidad, ya que ese punto (donde se produjo la divergencia) se considera como el punto de fallo crítico.

\begin{tabular}{lccc}
\cline { 2 - 4 } & AMBER & MORSE & $[115]$ ec. $(6)]$ \\
\cline { 2 - 4 } & $\lambda_{c r}(\mathrm{rad})$ & $\lambda_{c r}(\mathrm{rad})$ & $\lambda_{c r}(\mathrm{rad})$ \\
\hline $\mathrm{AC}(3,3)$ & 0.865 & 0.971 & 0.180 \\
$\mathrm{AC}(4,4)$ & 0.694 & 0.653 & 0.117 \\
\hline $\mathrm{ZZ}(5,0)$ & 0.958 & 1.162 & 0.210 \\
$\mathrm{ZZ}(7,0)$ & 0.706 & 0.831 & 0.115 \\
\hline $\mathrm{CH}(4,2)$ & 1.000 & 0.718 & 0.171 \\
$\mathrm{CH}(5,3)$ & 0.783 & 0.766 & 0.118 \\
\hline
\end{tabular}

Tabla 5.12: Rotaciones críticas de abolladura a torsión

Comparando valores de la tabla 5.12 se puede establecer que no existe una tendencia clara en $\lambda_{c r}$ al adoptar el potencial AMBER o Morse. Luego se puede concluir que el potencial interatómico no produce diferencias significativas respecto a las deformaciones críticas de abolladura, sin embargo el potencial Morse produce unas trayectorias de equilibrio algo más suaves (presentan menos quiebros) que el AMBER.

Respecto a las rotaciones críticas de abolladura, [115] obtiene $\lambda_{c r}=2.7$ rad para un nanotubo $\mathrm{ZZ}(13,0)$ de $1 \mathrm{~nm}$ de diámetro y $23 \mathrm{~nm}$ de longitud, la cual es comparable con los resultados obtenidos en la tabla 5.12 Incluso en el mismo trabajo se establece una validación de sus propios cálculos MD mediante modelos continuos a torsión: si $L \leq 136 d^{5 / 2}$ (cilindros cortos, lo que puede verificarse fácilmente en nuestro caso) la ec. (6) de [115] proporciona los resultados mostrados en la última columna de 5.12. Es evidente que sus valores son mucho menores que los obtenidos aquí porque los tubos simulados en la tesis a torsión son bastante más cortos y las coacciones circulares producidas por las condiciones de contorno en ambos extremos estabilizan la estructura contra la abolladura torsional (ver comentario 5.4.6).

\subsection{Sumario del análisis geométricamente no lineal}

Finalmente, recapitulamos los conceptos fundamentales extraídos de la extensión geométricamente no lineal de la formulación del modelo MSM stick-spiral:

1. En relación a los valores de las deformaciones críticas a compresión (ver tabla[5.2), el empleo del potencial AMBER o Morse en el análisis es prácticamente indiferente. Asimismo, aunque a flexión y torsión los valores obtenidos con cada potencial difieren en cierta medida (ver tablas 5.7 y 5.12), no se observan diferencias significativas. Por tanto, podemos concluir que la no linealidad constitutiva introducida por el potencial de Morse no influye apreciablemente en la respuesta a la inestabilidad. En consecuencia, la no linealidad geométrica de SWTNs se desarrolla previamente a la no linealidad constitutiva, de acuerdo con [27], [43], [94], [110].

2. Los cálculos atómicos desarrollados en este capítulo enfatizan que los modelos continuos no son fiables en el análisis geométricamente no lineal de SWNTs. Además de la controvertida elección de ciertos parámetros como el módulo de Young, el espesor de pared equivalente y la deformación por cortante (en modelos viga), los modelos continuos no pueden reproducir la estructura atómica de los nanotubos. En consecuencia, las imperfecciones (muy condicionantes en la respuesta frente a la inestabilidad) no están presentes en la geometría del modelo y se 
deben introducir indirectamente por medio de métodos artificiosos, lo que se traduce en una reducción importante de la precisión y resultados carentes de sentido físico.

3. Como se ha mostrado, nuestros resultados son razonables en comparación con algunos trabajos atómicos consultados (por ej. [43], [94], [109], [121]). Por otra parte, a pesar de la dudosa aplicabilidad de los modelos continuos a sistemas atómicos, algunos autores [115] los han empleado adoptando valores apropiados para algunos parámetros mecánicos con el objetivo de validar sus cálculos MD. En esta línea, hemos utilizado algunas de sus ecuaciones del continuo para comparar cualitativamente con nuestros resultados obtenidos, llegando a una coincidencia razonable a compresión (error relativo máximo alrededor del $20 \%$ ). No obstante, sólo se consigue una correspondencia en términos de orden de magnitud, a flexión y torsión.

4. Como resultados de salida de los códigos desarrollados se obtienen las trayectorias de equilibrio no lineales, así como geometrías deformadas, axiles de enlace, momentos angulares y reacciones de apoyo en la última iteración del último incremento. Respecto a los valores de las deformaciones críticas y las geometrías deformadas finales, las diferencias con otros trabajos publicados son debidas principalmente al relativamente reducido tamaño de los SWNTs simulados, a los efectos de borde causados por las coacciones circulares en ambos extremos y a la dispersión implícita producida por los datos de entrada del proceso incremental-iterativo sobre las deformaciones críticas de inestabilidad [11].

5. En cada iteración de los algoritmos numéricos desarrollados será necesario resolver dos sistemas lineales. Uno para obtener el vector velocidad incremental (5.62) y otro para resolver el incremento del campo de desplazamientos y del factor de carga (5.68). Esta tarea representa el mayor consumo en términos de número de operaciones y tiempo de ejecución del proceso, por tanto se idearon algunas mejoras de rendimiento en la subrutina diseñada para la resolución de sistemas lineales, adaptándola a matrices dispersas (como la matriz de rigidez tangente $\mathbf{K}\left(\mathbf{u}_{n}\right)$ ). Asimismo, se ha optimizado la cantidad de operaciones asociada al ensamblaje y reducción de las matrices de equilibrio y de rigidez, para que la resolución de los nanotubos ensayados sea en tiempos de ejecución razonables. 


\section{Capítulo 6}

\section{Aproximación energética a la geometría inicial del nanotubo}

En la sección 4.1.5 se puso de manifiesto la dificultad a la hora de elegir el sistema particular de esfuerzos iniciales que reproduzca la preenergía presente en el nanotubo, ya que existen infinitas combinaciones de esfuerzos autoequilibrados para un nanotubo dado.

En general, la determinación de estos esfuerzos iniciales está íntimamente relacionada con la estimación de la geometría inicial del SWNT. La forma más sencilla de proceder consiste en suponer una geometría inicial y calcular las deformaciones iniciales $\mathbf{e}_{0}$ y los esfuerzos asociados $\mathbf{p}_{0}$. De hecho, tanto en la literatura como en esta tesis se ha decidido aproximar la geometría inicial del nanotubo como cilíndrica y localizar en ésta los átomos de $\mathrm{C}$ mediante un mapeo conforme desde la lámina de grafeno plana. El motivo fundamental es la sencillez en la formulación analítica de este mapeo, sacrificando algo de precisión en virtud de una mayor simplicidad del problema.

Sin embargo, se plantea en este capítulo la búsqueda de geometrías iniciales más aproximadas a la situación real mediante una minimización energética previa a la actuación de las cargas externas. Aunque se vio en el capítulo4 que la introducción del pretensado no modifica significativamente la rigidez longitudinal del nanotubo, y se ha obviado su influencia en el capítulo 5, la aproximación a una geometría inicial más exacta deberá permitir estimar con mayor precisión los esfuerzos y deformaciones finales así como los puntos críticos asociados a fenómenos de inestabilidad.

Cabe esperar que los esfuerzos iniciales en la geometría inicial "mejorada" sean mucho menores que los estimados para el mapeo conforme. En concreto, si resultan suficientemente pequeños será posible despreciar el término $\mathbf{p}_{0}$ en la ecuación (4.6), dando lugar a una implementación numérica más sencilla dentro del marco de la linealidad.

La estimación de la geometría inicial se desarrolla en dos etapas. Primero, se ha supuesto que la geometría del nanotubo es un cilindro perfecto cuyo diámetro inicial debemos determinar. Segundo, se requerirá un nuevo mapeo para determinar las posiciones exactas de los átomos de carbono en la superficie de dicho cilindro. Esta última etapa queda fuera de los objetivos de la tesis como línea de investigación abierta (ver capítulo7).

El presente capítulo está destinado a la búsqueda del diámetro del SWNT que minimiza su energía total en ausencia de cargas externas, como mejora aproximativa a la geometría inicial.

\subsection{Hipótesis iniciales y potenciales simplificados}

Asumamos que la geometría inicial del nanotubo es un cilindro perfecto de diámetro $d_{0}$, nuestro objetivo consiste en minimizar la energía total del sistema definida por el potencial interatómico. Por 
coherencia, efectuamos esta minimización para dos potenciales diferentes (AMBER y Morse), cuyas expresiones recordamos en la tabla 6.1.

$$
\begin{array}{ll}
\text { AMBER } & U=\sum_{i} \frac{1}{2} k_{r}\left(\Delta r_{i}\right)^{2}+\sum_{j} \frac{1}{2} k_{\theta}\left(\Delta \theta_{j}\right)^{2} \\
\text { Morse } & U=\sum_{i} D_{e}\left\{\left[1-e^{-\beta\left(\Delta r_{i}\right)}\right]^{2}-1\right\}+\sum_{j} \frac{1}{2} k_{\theta}\left(\Delta \theta_{j}\right)^{2}\left[1+k_{s}\left(\Delta \theta_{j}\right)^{4}\right]
\end{array}
$$

Tabla 6.1: Potenciales interatómicos

donde las constantes implicadas en el potencial AMBER adoptan los valores (2.8) y las constantes incluidas en el Morse se definen en (2.19).

Teniendo en cuenta que la rigidez longitudinal de enlace es muy superior a la rigidez angular entre enlaces, la tendencia natural del sistema en ausencia de cargas externas será hacia una geometría en la que los ángulos entre enlaces cambien más que las distancias entre átomos, en comparación con la lámina de grafeno. Por tanto, supondremos en los desarrollos posteriores que los alargamientos de enlace desde la lámina de grafeno hasta la geometría inicial del nanotubo son nulos $\left(\Delta r_{i}=0\right)$. De esta forma, las expresiones simplificadas de los potenciales serán:

$$
\begin{array}{ll}
\text { AMBER } & U=\sum_{j} \frac{1}{2} k_{\theta}\left(\Delta \theta_{j}\right)^{2} \\
\text { Morse } & U=-\sum_{i} D_{e}+\sum_{j} \frac{1}{2} k_{\theta}\left(\Delta \theta_{j}\right)^{2}\left[1+k_{s}\left(\Delta \theta_{j}\right)^{4}\right]
\end{array}
$$

Expresando ahora las distorsiones angulares $\Delta \theta_{j}$ en función del diámetro del nanotubo, podemos escribir la función potencial en la forma $U=U(d)$. En estas condiciones, el diámetro inicial $d_{0}$ correspondiente a la geometría de equilibrio deberá verificar la condición de mínimo:

$$
\left[\frac{\partial U(d)}{\partial d}\right]_{d_{0}}=0
$$

Abordamos a continuación el desarrollo y resolución de la ecuación (6.3) para cada quiralidad independientemente.

\subsection{Determinación del diámetro inicial para SWNTs ZigZag}

Un nanotubo ZigZag con $\mathrm{m}$ celdas en la dirección transversal y supuesto de longitud infinita $\mathrm{ZZ}(\mathrm{m}, 0)$ posee simetría radial y se puede generar por repetición en la dirección longitudinal del sistema elemental representado en la figura 6.1 correspondiente a la posición de equilibrio buscada. En consecuencia, la minimización energética (6.3) puede llevarse a cabo sobre dicho sistema sin pérdida de generalidad.

Teniendo en cuenta que todos los enlaces en la posición de equilibrio miden $a_{0}$ (alargamiento nulo), podemos escribir:

$$
\begin{aligned}
l & =a_{0} \cos \alpha \\
\frac{l}{2} & =R \sin \left(\frac{\pi}{2 m}\right)
\end{aligned}
$$

donde:

$l \quad$ longitud de la proyección de los enlaces oblicuos sobre el plano transversal al eje

$\alpha$ ángulo comprendido entre los enlaces oblicuos y el plano transversal al eje 
Eliminando $l$ de 6.4), llegamos a:

$$
d_{0}=2 R=\frac{a_{0} \cos \alpha}{\sin \left(\frac{\pi}{2 m}\right)}
$$

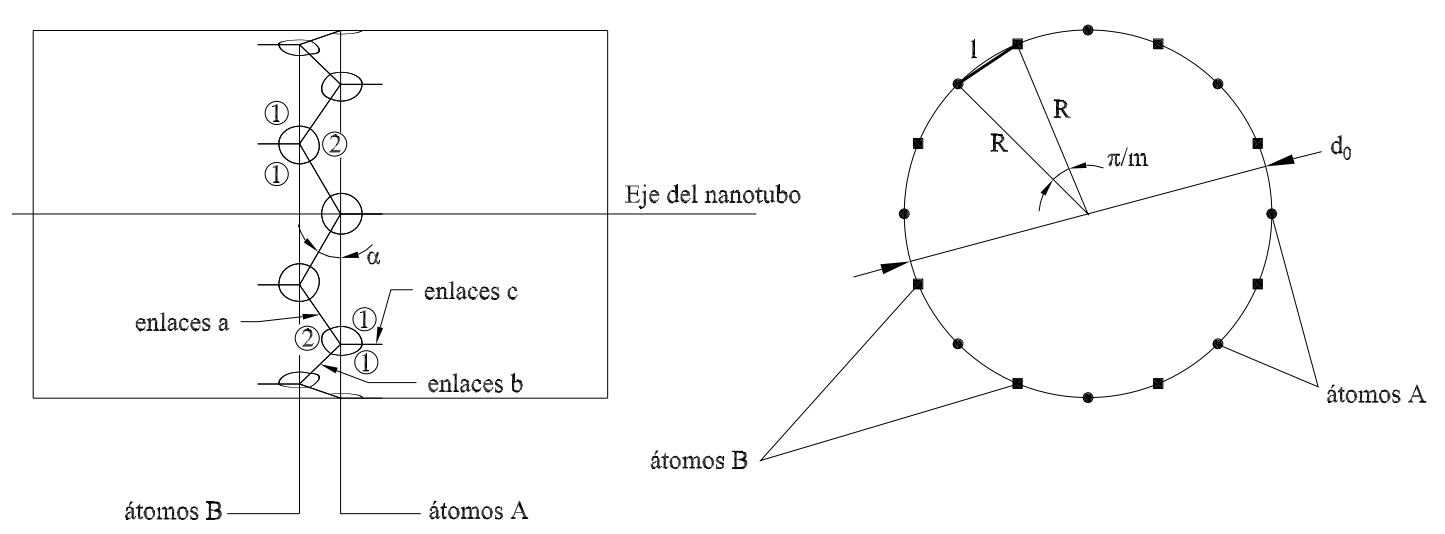

Figura 6.1: Célula representativa SWNTs ZigZag

En virtud de (6.5), adoptamos por comodidad $\alpha$ como parámetro independiente en el proceso de minimización. Teniendo en cuenta la distribución de enlaces representada en la figura 6.1, es inmediato observar que sólo existen dos ángulos tipo diferentes en nuestro sistema elemental. El primero de ellos, comprendido entre los enlaces ac y bc, será:

$$
\theta_{1}=\alpha+\frac{\pi}{2}
$$

En cuanto a la obtención de $\theta_{2}$, comprendido entre los enlaces ab, será algo más laboriosa y recurrimos a los esquemas vectoriales de la figura 6.2 entre tres átomos contiguos.

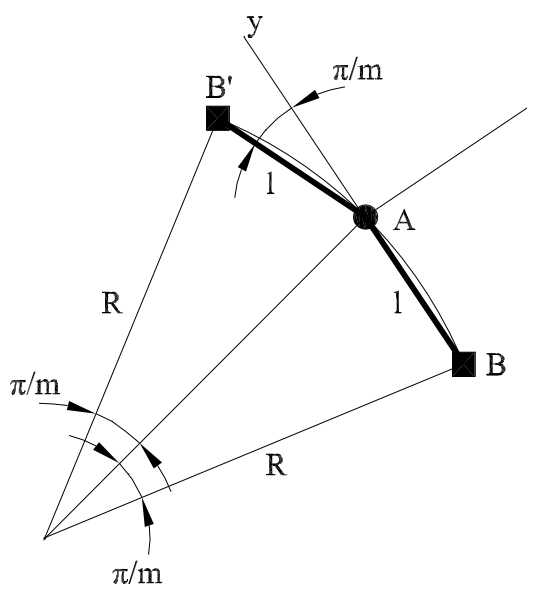

(a) Sección

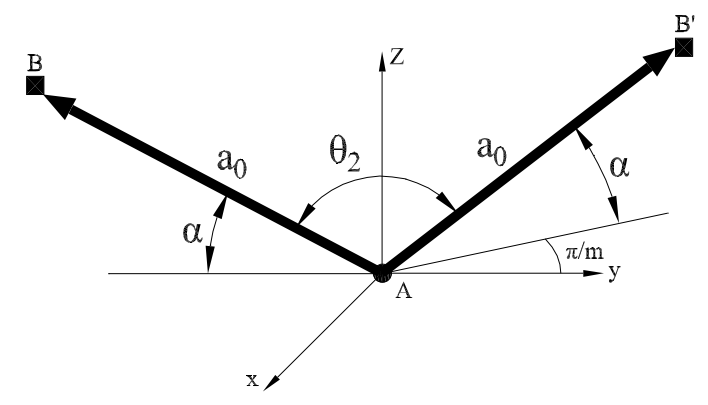

(b) Perspectiva

Figura 6.2: Esquemas vectoriales para obtener $\theta_{2}$ 
Nótese que el sistema de referencia auxiliar adoptado contiene el enlace AB en su plano $\{y z\}$. Por tanto, podemos introducir los siguientes vectores auxiliares:

$$
\begin{aligned}
& \mathbf{v}_{1}=\overrightarrow{A B}=\left\{0,-a_{0} \cos \alpha, a_{0} \sin \alpha\right\}^{T} \\
& \mathbf{v}_{2}=\overrightarrow{A B^{\prime}}=\left\{-a_{0} \cos \alpha \sin \left(\frac{\pi}{m}\right), a_{0} \cos \alpha \cos \left(\frac{\pi}{m}\right), a_{0} \sin \alpha\right\}^{T}
\end{aligned}
$$

obteniendo el ángulo que forman a partir de su producto escalar:

$$
\mathbf{v}_{1}^{T} \mathbf{v}_{2}=\left|\mathbf{v}_{1}\right|\left|\mathbf{v}_{2}\right| \cos \theta_{2}
$$

Sustituyendo los vectores 6.7 en 6.8 llegamos a:

$$
\cos \theta_{2}=\sin ^{2} \alpha\left(1+\cos \frac{\pi}{m}\right)-\cos \frac{\pi}{m}
$$

Empleando ahora (6.6) y (6.9), obtenemos las siguientes distorsiones angulares en comparación con los ángulos observados en la lámina de grafeno:

$$
\begin{aligned}
& \Delta \theta_{1}=\alpha-\frac{\pi}{6} \\
& \Delta \theta_{2}=\arccos \left[\sin ^{2} \alpha\left(1+C_{m}\right)-C_{m}\right]-\frac{2 \pi}{3}
\end{aligned}
$$

donde hemos denominado:

$$
C_{m}=\cos \left(\frac{\pi}{m}\right)
$$

\subsubsection{Desarrollo con potencial AMBER}

Particularizamos la expresión (6.1) del potencial AMBER para nuestro sistema elemental (fig. 6.1), que comprende $2 m$ ángulos del tipo 2 y $4 m$ ángulos de tipo 1, según:

$$
U=m k_{\theta}\left(\Delta \theta_{2}\right)^{2}+2 m k_{\theta}\left(\Delta \theta_{1}\right)^{2}
$$

Forzando la condición de mínimo 6.3 sobre 6.12

$$
m k_{\theta}\left(2 \Delta \theta_{2} \frac{\partial \Delta \theta_{2}}{\partial \alpha}+4 \Delta \theta_{1} \frac{\partial \Delta \theta_{1}}{\partial \alpha}\right)=0
$$

sustituyendo (6.10) y operando, queda:

$$
-\left\{\arccos \left[\sin ^{2} \alpha\left(1+C_{m}\right)-C_{m}\right]-\frac{2 \pi}{3}\right\} \frac{\left(1+C_{m}\right) \sin \alpha \cos \alpha}{\sqrt{1-\left[\sin ^{2} \alpha\left(1+C_{m}\right)-C_{m}\right]^{2}}}+\left(\alpha-\frac{\pi}{6}\right)=0
$$

ecuación implícita en $\alpha$ que se puede resolver numéricamente por el método de Newton (por ejemplo). Obtenido $\alpha$ y sustituyendo en (6.5), se llega a los valores resumidos en la tabla 6.2 para $a_{0}=$ $0.142 \mathrm{~nm}$. Se incluyen asimismo los diámetros calculados con el mapeo conforme [26] a modo de comparación.

Como puede comprobarse, el diámetro obtenido mediante la minimización de la energía $d_{0}$ es ligeramente superior al calculado con el mapeo conforme $D_{t}$, como cabía esperar tras estudiar el efecto del pretensado introducido en el modelo MSM (ver sección 4.4).

Con el objetivo de comparar los esfuerzos (y diámetros) del presente proceso energético con los resultados numéricos obtenidos para el análisis lineal mediante el modelo MSM sin cargas externas, 
6.2. Determinación del diámetro inicial para SWNTs ZigZag

\begin{tabular}{lcccc}
\hline & $\mathrm{m}$ & $D_{t}(\mathrm{~nm})(1.1)$ & $d_{0}(\mathrm{~nm}) \underline{6.5}$ & $\alpha(\mathrm{rad})(\underline{6.14})$ \\
\hline $\mathrm{ZZ}(4,0)$ & 4 & 0.3132 & 0.3356 & 0.4406 \\
$\mathrm{ZZ}(5,0)$ & 5 & 0.3914 & 0.4100 & 0.4685 \\
$\mathrm{ZZ}(6,0)$ & 6 & 0.4697 & 0.4854 & 0.4848 \\
$\mathrm{ZZ}(10,0)$ & 10 & 0.7829 & 0.7925 & 0.5094 \\
$\mathrm{ZZ}(15,0)$ & 15 & 1.1743 & 1.1808 & 0.5173 \\
$\mathrm{ZZ}(20,0)$ & 20 & 1.5658 & 1.5706 & 0.5200 \\
\hline
\end{tabular}

Tabla 6.2: Diámetros iniciales ZigZag, AMBER

calculamos los esfuerzos como sigue. Por hipótesis, los axiles de barra serán nulos $\left(n_{0}^{a}=n_{0}^{b}=n_{0}^{c}=0\right)$. Por otra parte, los momentos de muelle se obtienen evaluando (6.10) y sustituyendo en:

$$
\begin{aligned}
& m_{0}^{1}=-k_{\theta} \Delta \theta_{1} \\
& m_{0}^{2}=-k_{\theta} \Delta \theta_{2}
\end{aligned}
$$

Resumimos en las tablas 6.3 y 6.4 los resultados obtenidos:

\begin{tabular}{lccccl}
\hline & $n_{0}^{a}(\mathrm{nN})$ & $n_{0}^{b}(\mathrm{nN})$ & $n_{0}^{c}(\mathrm{nN})$ & $m_{0}^{1}(\mathrm{nNnm})$ & $m_{0}^{2}(\mathrm{nNnm})$ \\
\hline $\mathrm{ZZ}(4,0)$ & 0 & 0 & 0 & $7.2731 \cdot 10^{-2}$ & $1.0139 \cdot 10^{-1}$ \\
$\mathrm{ZZ}(5,0)$ & 0 & 0 & 0 & $4.8247 \cdot 10^{-2}$ & $5.9438 \cdot 10^{-2}$ \\
$\mathrm{ZZ}(6,0)$ & 0 & 0 & 0 & $3.3988 \cdot 10^{-2}$ & $3.9206 \cdot 10^{-2}$ \\
$\mathrm{ZZ}(10,0)$ & 0 & 0 & 0 & $1.2415 \cdot 10^{-2}$ & $1.3054 \cdot 10^{-2}$ \\
$\mathrm{ZZ}(15,0)$ & 0 & 0 & 0 & $5.5350 \cdot 10^{-3}$ & $5.6587 \cdot 10^{-3}$ \\
$\mathrm{ZZ}(20,0)$ & 0 & 0 & 0 & $3.1164 \cdot 10^{-3}$ & $3.1552 \cdot 10^{-3}$ \\
\hline
\end{tabular}

Tabla 6.3: Esfuerzos minimización energética ZZ, AMBER

\begin{tabular}{lcccccc}
\hline & $d_{0}(\mathrm{~nm})$ & $n_{0}^{a}(\mathrm{nN})$ & $n_{0}^{b}(\mathrm{nN})$ & $n_{0}^{c}(\mathrm{nN})$ & $m_{0}^{1}(\mathrm{nNnm})$ & $m_{0}^{2}(\mathrm{nNnm})$ \\
\hline $\mathrm{ZZ}(4,0)$ & 0.3370 & $6.08 \cdot 10^{-6}$ & $4.82 \cdot 10^{-6}$ & $1.26 \cdot 10^{-6}$ & $7.3818 \cdot 10^{-2}$ & $9.5129 \cdot 10^{-2}$ \\
$\mathrm{ZZ}(5,0)$ & 0.3935 & $5.91 \cdot 10^{-6}$ & $4.51 \cdot 10^{-6}$ & $4.12 \cdot 10^{-6}$ & $4.8434 \cdot 10^{-2}$ & $5.7563 \cdot 10^{-2}$ \\
$\mathrm{ZZ}(6,0)$ & 0.4858 & $4.77 \cdot 10^{-6}$ & $4.41 \cdot 10^{-6}$ & $1.73 \cdot 10^{-6}$ & $3.4021 \cdot 10^{-2}$ & $3.8539 \cdot 10^{-2}$ \\
$\mathrm{ZZ}(10,0)$ & 0.7928 & $5.09 \cdot 10^{-6}$ & $3.40 \cdot 10^{-6}$ & $-5.61 \cdot 10^{-6}$ & $1.2399 \cdot 10^{-2}$ & $1.3027 \cdot 10^{-2}$ \\
$\mathrm{ZZ}(15,0)$ & 1.1808 & $4.87 \cdot 10^{-6}$ & $3.69 \cdot 10^{-6}$ & $-5.05 \cdot 10^{-6}$ & $5.5211 \cdot 10^{-3}$ & $5.6624 \cdot 10^{-3}$ \\
$\mathrm{ZZ}(20,0)$ & 1.5707 & $4.54 \cdot 10^{-6}$ & $5.11 \cdot 10^{-6}$ & $1.78 \cdot 10^{-6}$ & $3.1090 \cdot 10^{-3}$ & $3.1584 \cdot 10^{-3}$ \\
\hline
\end{tabular}

Tabla 6.4: Esfuerzos modelo MSM (ZZ análisis lineal, AMBER)

Como se puede observar, los axiles obtenidos con el modelo MSM son insignificantes. Tomando ahora como solución exacta de referencia la correspondiente al proceso minimizador, resumimos a en la tabla 6.5 los errores relativos para el diámetro y los momentos angulares respecto al modelo MSM. 


\begin{tabular}{lccc}
\hline & $\varepsilon_{d}(\%)$ & $\varepsilon_{m^{1}}(\%)$ & $\varepsilon_{m^{2}}(\%)$ \\
\hline $\mathrm{ZZ}(4,0)$ & 0.4082 & 1.4955 & 6.1761 \\
$\mathrm{ZZ}(5,0)$ & 0.5252 & 0.3874 & 3.1545 \\
$\mathrm{ZZ}(6,0)$ & 0.0773 & 0.0962 & 1.7000 \\
$\mathrm{ZZ}(10,0)$ & 0.0418 & 0.1297 & 0.2007 \\
$\mathrm{ZZ}(15,0)$ & 0.0042 & 0.2524 & 0.0652 \\
$\mathrm{ZZ}(20,0)$ & 0.0070 & 0.2359 & 0.1002 \\
\hline
\end{tabular}

Tabla 6.5: Errores relativos respecto a la minimización energética ZZ, AMBER

Se pone de manifiesto la excelente correspondencia entre ambos procedimientos, con un error máximo relativo del $0.53 \%$ en el diámetro y del $6.18 \%$ en los momentos angulares.

\subsubsection{Desarrollo con potencial Morse}

La expresión (6.2) del potencial Morse reducida al sistema elemental de la figura 6.1 se puede escribir:

$$
U=-4 m D_{e}+m k_{\theta}\left(\Delta \theta_{2}\right)^{2}\left[1+k_{s}\left(\Delta \theta_{2}\right)^{4}\right]+2 m k_{\theta}\left(\Delta \theta_{1}\right)^{2}\left[1+k_{s}\left(\Delta \theta_{1}\right)^{4}\right]
$$

Aplicando la condición de mínimo 6.3 a 6.16 y empleando 6.10 se alcanza:

$$
-\Delta \theta_{2}\left[1+3 k_{s}\left(\Delta \theta_{2}\right)^{4}\right] \frac{\left(1+C_{m}\right) \sin \alpha \cos \alpha}{\sqrt{1-\left[\sin ^{2} \alpha\left(1+C_{m}\right)-C_{m}\right]^{2}}}+\Delta \theta_{1}\left[1+3 k_{s}\left(\Delta \theta_{1}\right)^{4}\right]=0
$$

donde $\Delta \theta_{1}, \Delta \theta_{2}$ están en función de $\alpha$ a través de (6.10), de forma que la ecuación (6.17) es implícita en $\alpha$. Resolviéndola numéricamente para $a_{0}=0.142 \mathrm{~nm}$ y sustituyendo en [6.5), obtenemos los valores de la tabla 6.6 junto con los calculados mediante el mapeo conforme.

\begin{tabular}{lcccc}
\hline & $\mathrm{m}$ & $D_{t}(\mathrm{~nm})(1.1)$ & $d_{0}(\mathrm{~nm}) \sqrt[6.5]{6}$ & $\alpha(\mathrm{rad})(\underline{6.17}$ \\
\hline $\mathrm{ZZ}(4,0)$ & 4 & 0.3132 & 0.3356 & 0.4406 \\
$\mathrm{ZZ}(5,0)$ & 5 & 0.3914 & 0.4100 & 0.4685 \\
$\mathrm{ZZ}(6,0)$ & 6 & 0.4697 & 0.4854 & 0.4848 \\
$\mathrm{ZZ}(10,0)$ & 10 & 0.7829 & 0.7925 & 0.5094 \\
$\mathrm{ZZ}(15,0)$ & 15 & 1.1743 & 1.1808 & 0.5173 \\
$\mathrm{ZZ}(20,0)$ & 20 & 1.5658 & 1.5706 & 0.5200 \\
\hline
\end{tabular}

Tabla 6.6: Diámetros iniciales ZigZag, Morse

Comparando valores con la tabla 6.2 se puede concluir que el potencial Morse no introduce ninguna diferencia en el diámetro inicial en ausencia en cargas externas.

Analizamos también en este caso las posibles diferencias entre este procedimiento de minimización y los resultados obtenidos con el modelo MSM (análisis lineal) en ausencia de cargas externas. Los axiles siguen siendo nulos $\left(n_{0}^{a}=n_{0}^{b}=n_{0}^{c}=0\right)$ por hipótesis, y los momentos se calculan empleando la relación (4.13b) que recordamos a continuación:

$$
\begin{aligned}
& m_{0}^{1}=k_{\theta}\left(\Delta \theta_{1}\right)\left[1+3 k_{s}\left(\Delta \theta_{1}\right)^{4}\right] \\
& m_{0}^{2}=k_{\theta}\left(\Delta \theta_{2}\right)\left[1+3 k_{s}\left(\Delta \theta_{2}\right)^{4}\right]
\end{aligned}
$$

Resumimos en las tablas 6.7 y 6.8 los resultados obtenidos: 
6.3. Determinación del diámetro inicial para SWNTs Armchair

\begin{tabular}{lccccc}
\hline & $n_{0}^{a}(\mathrm{nN})$ & $n_{0}^{b}(\mathrm{nN})$ & $n_{0}^{c}(\mathrm{nN})$ & $m_{0}^{1}(\mathrm{nNnm})$ & $m_{0}^{2}(\mathrm{nNnm})$ \\
\hline $\mathrm{ZZ}(4,0)$ & 0 & 0 & 0 & $7.4725 \cdot 10^{-2}$ & $1.0417 \cdot 10^{-1}$ \\
$\mathrm{ZZ}(5,0)$ & 0 & 0 & 0 & $4.9559 \cdot 10^{-2}$ & $6.1055 \cdot 10^{-2}$ \\
$\mathrm{ZZ}(6,0)$ & 0 & 0 & 0 & $3.4912 \cdot 10^{-2}$ & $4.0271 \cdot 10^{-2}$ \\
$\mathrm{ZZ}(10,0)$ & 0 & 0 & 0 & $1.2753 \cdot 10^{-2}$ & $1.3408 \cdot 10^{-2}$ \\
$\mathrm{ZZ}(15,0)$ & 0 & 0 & 0 & $5.6854 \cdot 10^{-3}$ & $5.8124 \cdot 10^{-3}$ \\
$\mathrm{ZZ}(20,0)$ & 0 & 0 & 0 & $3.2010 \cdot 10^{-3}$ & $3.2409 \cdot 10^{-3}$ \\
\hline
\end{tabular}

Tabla 6.7: Esfuerzos minimización energética ZZ, Morse

\begin{tabular}{lcccccc}
\hline & $d_{0}(\mathrm{~nm})$ & $n_{0}^{a}(\mathrm{nN})$ & $n_{0}^{b}(\mathrm{nN})$ & $n_{0}^{c}(\mathrm{nN})$ & $m_{0}^{1}(\mathrm{nNnm})$ & $m_{0}^{2}(\mathrm{nNnm})$ \\
\hline $\mathrm{ZZ}(4,0)$ & 0.3361 & $1.18 \cdot 10^{-4}$ & $1.18 \cdot 10^{-4}$ & $2.27 \cdot 10^{-6}$ & $7.7617 \cdot 10^{-2}$ & $1.0002 \cdot 10^{-1}$ \\
$\mathrm{ZZ}(5,0)$ & 0.4102 & $2.19 \cdot 10^{-5}$ & $2.19 \cdot 10^{-5}$ & $3.61 \cdot 10^{-6}$ & $5.0323 \cdot 10^{-2}$ & $5.9809 \cdot 10^{-2}$ \\
$\mathrm{ZZ}(6,0)$ & 0.4855 & $4.42 \cdot 10^{-5}$ & $4.42 \cdot 10^{-5}$ & $2.23 \cdot 10^{-6}$ & $3.5162 \cdot 10^{-2}$ & $3.9829 \cdot 10^{-2}$ \\
$\mathrm{ZZ}(10,0)$ & 0.7925 & $3.74 \cdot 10^{-5}$ & $3.74 \cdot 10^{-5}$ & $-3.35 \cdot 10^{-6}$ & $1.2754 \cdot 10^{-2}$ & $1.3391 \cdot 10^{-2}$ \\
$\mathrm{ZZ}(15,0)$ & 1.1807 & $6.56 \cdot 10^{-6}$ & $6.56 \cdot 10^{-6}$ & $2.01 \cdot 10^{-7}$ & $5.6760 \cdot 10^{-3}$ & $5.8156 \cdot 10^{-3}$ \\
$\mathrm{ZZ}(20,0)$ & 1.5706 & $4.65 \cdot 10^{-6}$ & $4.65 \cdot 10^{-6}$ & $-1.78 \cdot 10^{-7}$ & $3.1937 \cdot 10^{-3}$ & $3.2444 \cdot 10^{-3}$ \\
\hline
\end{tabular}

Tabla 6.8: Esfuerzos modelo MSM (ZZ análisis lineal, Morse)

En este caso los axiles obtenidos son algo superiores al potencial AMBER, pero siguen siendo despreciables. Se detallan a continuación los errores relativos para el diámetro y los momentos angulares, producto de la comparación entre la minimización energética (adoptada como solución exacta) y los resultados del modelo MSM:

\begin{tabular}{lccc}
\hline & $\varepsilon_{d}(\%)$ & $\varepsilon_{m^{1}}(\%)$ & $\varepsilon_{m^{2}}(\%)$ \\
\hline $\mathrm{ZZ}(4,0)$ & 0.1394 & 3.8691 & 3.9838 \\
$\mathrm{ZZ}(5,0)$ & 0.0483 & 1.5408 & 2.0419 \\
$\mathrm{ZZ}(6,0)$ & 0.0155 & 0.7158 & 1.0973 \\
$\mathrm{ZZ}(10,0)$ & 0.0039 & 0.0102 & 0.1298 \\
$\mathrm{ZZ}(15,0)$ & 0.0042 & 0.1666 & 0.0549 \\
$\mathrm{ZZ}(20,0)$ & 0.0006 & 0.2290 & 0.1065 \\
\hline
\end{tabular}

Tabla 6.9: Errores relativos respecto a la minimización energética ZZ, Morse

Queda patente la extrema coincidencia entre los resultados de ambos procedimientos, con un error máximo relativo del $0.14 \%$ en el diámetro y del $4 \%$ en los momentos angulares.

\subsection{Determinación del diámetro inicial para SWNTs Armchair}

En la figura 6.3 se representa el sistema unitario que permite generar por repetición longitudinal un nanotubo $\mathrm{AC}(\mathrm{m}, \mathrm{m})$ con $2 m$ celdas en la dirección transversal. Buscamos entonces la geometría inicial de equilibrio para el nanotubo minimizando la energía potencial de dicho sistema. 


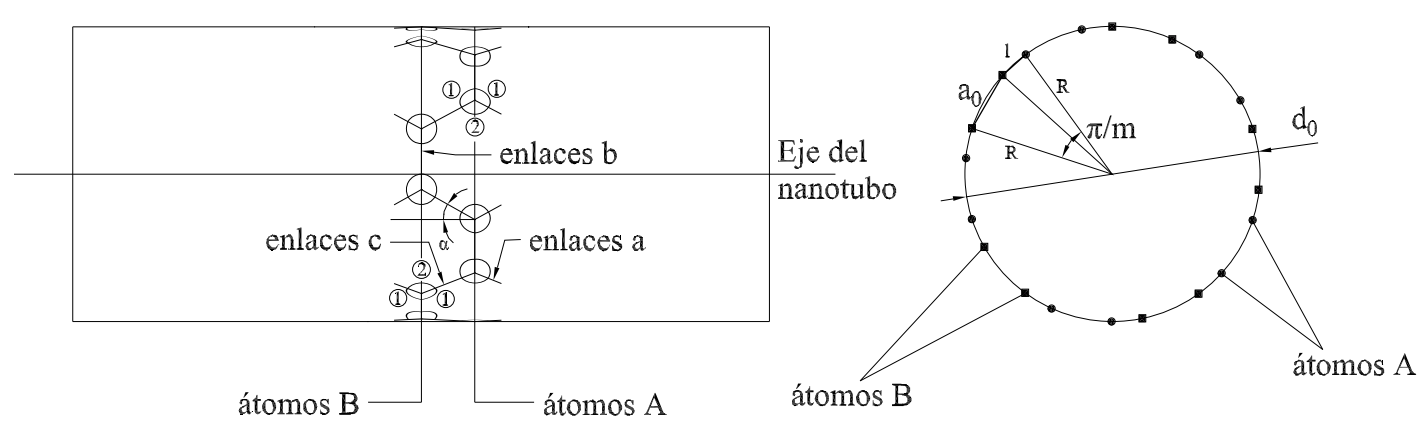

Figura 6.3: Célula representativa SWNTs Armchair

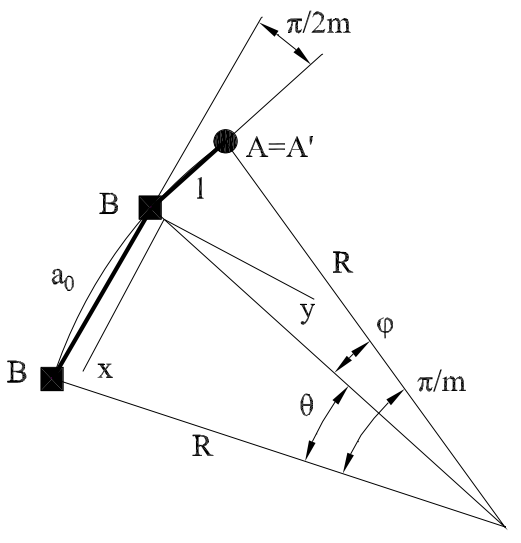

(a) Sección

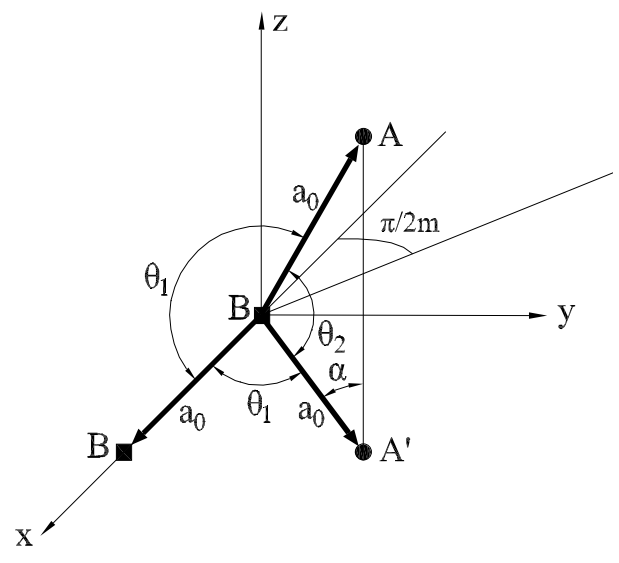

(b) Perspectiva

Figura 6.4: Esquemas vectoriales para obtener $\theta_{1}, \theta_{2}$

A partir de la figura 6.3 y del esquema vectorial $6.4(\mathrm{a})$, se pueden establecer las siguientes relaciones:

$$
\begin{gathered}
\quad l=a_{0} \sin \alpha \\
\frac{a_{0}}{d_{0}}=\sin \frac{\theta}{2} ; \quad \sqrt{1-\left(\frac{a_{0}}{d_{0}}\right)^{2}}=\cos \frac{\theta}{2} \\
\frac{l}{d_{0}}=\sin \frac{\varphi}{2} ; \quad \sqrt{1-\left(\frac{l}{d_{0}}\right)^{2}}=\cos \frac{\varphi}{2}
\end{gathered}
$$

Con el objetivo de relacionar el diámetro $d_{0}$ con la orientación $\alpha$ establecemos:

$$
\begin{aligned}
& \sin \left(\frac{\theta}{2}+\frac{\varphi}{2}\right)=\sin \frac{\theta}{2} \cos \frac{\varphi}{2}+\sin \frac{\varphi}{2} \cos \frac{\theta}{2}=\sin \frac{\pi}{2 m} \\
& \cos \left(\frac{\theta}{2}+\frac{\varphi}{2}\right)=\cos \frac{\theta}{2} \cos \frac{\varphi}{2}-\sin \frac{\varphi}{2} \sin \frac{\theta}{2}=\cos \frac{\pi}{2 m}
\end{aligned}
$$


Elevando 6.20) al cuadrado y sustituyendo 6.21, se llega a:

$$
\sin ^{2} \frac{\theta}{2} \cos ^{2} \frac{\varphi}{2}+\cos ^{2} \frac{\theta}{2} \sin ^{2} \frac{\varphi}{2}+2 \sin ^{2} \frac{\theta}{2} \sin ^{2} \frac{\varphi}{2}+2 \sin \frac{\theta}{2} \sin \frac{\varphi}{2} \cos \frac{\pi}{2 m}=\sin ^{2} \frac{\pi}{2 m}
$$

empleando adecuadamente (6.19) en 6.22) y operando, obtenemos:

$$
d_{0}=\frac{a_{0}}{\sin \frac{\pi}{2 m}} \sqrt{1+\sin ^{2} \alpha+2 \sin \alpha \cos \frac{\pi}{2 m}}
$$

Denotando ahora:

$$
S_{2 m}=\sin \frac{\pi}{2 m} \quad C_{2 m}=\cos \frac{\pi}{2 m}
$$

se llega a la siguiente relación del diámetro con la orientación $\alpha$, algo más compacta:

$$
d_{0}=\frac{a_{0}}{S_{2 m}} \sqrt{1+\sin ^{2} \alpha+2 \sin \alpha C_{2 m}}
$$

Teniendo en cuenta la relación 6.25), adoptamos como parámetro independiente el ángulo $\alpha$ en el proceso de minimización para los nanotubos Armchair. Por tanto, cabe obtener las expresiones de las variaciones angulares $\Delta \theta_{1}, \Delta \theta_{2}$ en función de dicho parámetro. Para ello, recurrimos a los esquemas vectoriales de la figura 6.4 e introducimos los siguientes vectores auxiliares:

$$
\begin{aligned}
& \mathbf{w}_{1}=\overrightarrow{B B}=\left\{a_{0}, 0,0\right\}^{T} \\
& \mathbf{w}_{2}=\overrightarrow{B A}=\left\{-a_{0} \sin \alpha C_{2 m}, a_{0} \sin \alpha S_{2 m}, a_{0} \cos \alpha\right\}^{T} \\
& \mathbf{w}_{3}=\overrightarrow{B A^{\prime}}=\left\{-a_{0} \sin \alpha C_{2 m}, a_{0} \sin \alpha S_{2 m},-a_{0} \cos \alpha\right\}^{T}
\end{aligned}
$$

Análogamente al caso ZigZag (ec (6.8)), planteamos los siguientes productos escalares:

$$
\begin{aligned}
& \mathbf{w}_{1}^{T} \mathbf{w}_{2}=\left|\mathbf{w}_{1}\right|\left|\mathbf{w}_{2}\right| \cos \theta_{1} \\
& \mathbf{w}_{2}^{T} \mathbf{w}_{3}=\left|\mathbf{w}_{2}\right|\left|\mathbf{w}_{3}\right| \cos \theta_{2}
\end{aligned}
$$

empleando las definiciones 6.26) en 6.27)

$$
\begin{aligned}
& \cos \theta_{1}=-C_{2 m} \sin \alpha \\
& \cos \theta_{2}=-\cos 2 \alpha
\end{aligned}
$$

aunque es evidente que 6.28b implica:

$$
\theta_{2}=\pi-2 \alpha
$$

Por tanto, las distorsiones angulares para SWNTs Armchair se escriben:

$$
\begin{aligned}
& \Delta \theta_{1}=\arccos \left[-C_{2 m} \sin \alpha\right]-\frac{2 \pi}{3} \\
& \Delta \theta_{2}=\frac{\pi}{3}-2 \alpha
\end{aligned}
$$




\subsubsection{Desarrollo con potencial AMBER}

El sistema elemental de la figura 6.3 contiene $4 m$ ángulos de tipo 2 y $8 m$ ángulos de tipo 1 , de forma que la expresión (6.1) del potencial AMBER se reduce a:

$$
U=2 m k_{\theta}\left(\Delta \theta_{2}\right)^{2}+4 m k_{\theta}\left(\Delta \theta_{1}\right)^{2}=2 m k_{\theta}\left(\Delta \theta_{2}^{2}+2 \Delta \theta_{1}^{2}\right)
$$

Forzando la condición de mínimo 6.3 sobre 6.31

$$
2 m k_{\theta}\left(2 \Delta \theta_{2} \frac{\partial \Delta \theta_{2}}{\partial \alpha}+4 \Delta \theta_{1} \frac{\partial \Delta \theta_{1}}{\partial \alpha}\right)=0
$$

sustituyendo 6.30 y operando, queda:

$$
-\left(\frac{\pi}{3}-2 \alpha\right)+\left(\arccos \left[-C_{2 m} \sin \alpha\right]-\frac{2 \pi}{3}\right) \frac{C_{2 m} \cos \alpha}{\sqrt{1-\left(C_{2 m} \sin \alpha\right)^{2}}}=0
$$

ecuación implícita en $\alpha$ que resolveremos numéricamente. Obtenido $\alpha$, computamos el diámetro buscado $d_{0}$ mediante 6.25) para $a_{0}=0.142 \mathrm{~nm}$. Un resumen de los valores obtenidos en comparación con los correspondientes al mapeo conforme se incluye en la tabla 6.10. Como cabía esperar, para los nanotubos AC también se obtiene un diámetro $d_{0}$ ligeramente superior al calculado con el mapeo conforme $D_{t}$.

\begin{tabular}{lcccc}
\hline & $\mathrm{m}$ & $D_{t}(\mathrm{~nm})(1.1)$ & $d_{0}(\mathrm{~nm})[6.25)$ & $\alpha(\mathrm{rad})[6.33$ \\
\hline $\mathrm{AC}(3,3)$ & 3 & 0.4068 & 0.4185 & 0.5470 \\
$\mathrm{AC}(4,4)$ & 4 & 0.5424 & 0.5514 & 0.5375 \\
$\mathrm{AC}(5,5)$ & 5 & 0.6780 & 0.6853 & 0.5327 \\
$\mathrm{AC}(10,10)$ & 10 & 1.3560 & 1.3597 & 0.5260 \\
$\mathrm{AC}(15,15)$ & 15 & 2.0340 & 2.0365 & 0.5246 \\
$\mathrm{AC}(20,20)$ & 20 & 2.7120 & 2.7139 & 0.5242 \\
\hline
\end{tabular}

Tabla 6.10: Diámetros iniciales Armchair, AMBER

Comparamos a continuación (tablas 6.11 y 6.12 los resultados obtenidos con los del modelo MSM (análisis lineal) en ausencia de cargas externas. Para el presente procedimiento minimizador, los axiles se consideran nulos por hipótesis y los momentos se evalúan empleando (6.15).

\begin{tabular}{lccccc}
\hline & $n_{0}^{a}(\mathrm{nN})$ & $n_{0}^{b}(\mathrm{nN})$ & $n_{0}^{c}(\mathrm{nN})$ & $m_{0}^{1}(\mathrm{nNnm})$ & $m_{0}^{2}(\mathrm{nNnm})$ \\
\hline $\mathrm{AC}(3,3)$ & 0 & 0 & 0 & $4.9386 \cdot 10^{-2}$ & $4.0915 \cdot 10^{-2}$ \\
$\mathrm{AC}(4,4)$ & 0 & 0 & 0 & $2.7052 \cdot 10^{-2}$ & $2.4367 \cdot 10^{-2}$ \\
$\mathrm{AC}(5,5)$ & 0 & 0 & 0 & $1.7080 \cdot 10^{-2}$ & $1.5981 \cdot 10^{-2}$ \\
$\mathrm{AC}(10,10)$ & 0 & 0 & 0 & $4.1880 \cdot 10^{-3}$ & $4.1194 \cdot 10^{-3}$ \\
$\mathrm{AC}(15,15)$ & 0 & 0 & 0 & $1.8544 \cdot 10^{-3}$ & $1.8408 \cdot 10^{-3}$ \\
$\mathrm{AC}(20,20)$ & 0 & 0 & 0 & $1.0417 \cdot 10^{-3}$ & $1.0374 \cdot 10^{-3}$ \\
\hline
\end{tabular}

Tabla 6.11: Esfuerzos minimización energética AC, AMBER 
6.3. Determinación del diámetro inicial para SWNTs Armchair

\begin{tabular}{lcccccc}
\hline & $d_{0}(\mathrm{~nm})$ & $n_{0}^{a}(\mathrm{nN})$ & $n_{0}^{b}(\mathrm{nN})$ & $n_{0}^{c}(\mathrm{nN})$ & $m_{0}^{1}(\mathrm{nNnm})$ & $m_{0}^{2}(\mathrm{nNnm})$ \\
\hline $\mathrm{AC}(3,3)$ & 0.4185 & $-8,62 \cdot 10^{-6}$ & $-2,66 \cdot 10^{-5}$ & $-8,62 \cdot 10^{-6}$ & $4.9299 \cdot 10^{-2}$ & $4.1024 \cdot 10^{-2}$ \\
$\mathrm{AC}(4,4)$ & 0.5514 & $6,19 \cdot 10^{-6}$ & $9,78 \cdot 10^{-6}$ & $6,19 \cdot 10^{-6}$ & $2.7039 \cdot 10^{-2}$ & $2.4384 \cdot 10^{-2}$ \\
$\mathrm{AC}(5,5)$ & 0.6853 & $2,64 \cdot 10^{-6}$ & $8,69 \cdot 10^{-6}$ & $2,64 \cdot 10^{-6}$ & $1.7083 \cdot 10^{-2}$ & $1.5980 \cdot 10^{-2}$ \\
$\mathrm{AC}(10,10)$ & 1.3597 & $5,90 \cdot 10^{-6}$ & $6,58 \cdot 10^{-6}$ & $5,90 \cdot 10^{-6}$ & $4.2070 \cdot 10^{-3}$ & $4.1065 \cdot 10^{-3}$ \\
$\mathrm{AC}(15,15)$ & 2.0365 & $6,97 \cdot 10^{-6}$ & $6,60 \cdot 10^{-6}$ & $6,97 \cdot 10^{-6}$ & $1.8724 \cdot 10^{-3}$ & $1.8289 \cdot 10^{-3}$ \\
$\mathrm{AC}(20,20)$ & 2.7139 & $4,69 \cdot 10^{-6}$ & $6,27 \cdot 10^{-6}$ & $4,69 \cdot 10^{-6}$ & $1.0617 \cdot 10^{-3}$ & $1.0251 \cdot 10^{-3}$ \\
\hline
\end{tabular}

Tabla 6.12: Esfuerzos modelo MSM (AC análisis lineal, AMBER)

Como se puede comprobar, los axiles obtenidos con el modelo MSM son claramente despreciables. Se detallan a continuación los errores relativos para el diámetro y los momentos angulares, producto de la comparación entre la minimización energética (adoptada como solución exacta) y los resultados del modelo MSM:

\begin{tabular}{lccc}
\hline & $\varepsilon_{d}(\%)$ & $\varepsilon_{m^{1}}(\%)$ & $\varepsilon_{m^{2}}(\%)$ \\
\hline $\mathrm{AC}(3,3)$ & 0.0119 & 0.1764 & 0.2654 \\
$\mathrm{AC}(4,4)$ & 0.0040 & 0.0447 & 0.0706 \\
$\mathrm{AC}(5,5)$ & 0.0016 & 0.0199 & 0.0019 \\
$\mathrm{AC}(10,10)$ & 0.0004 & 0.4542 & 0.3132 \\
$\mathrm{AC}(15,15)$ & 0.0005 & 0.9739 & 0.6481 \\
$\mathrm{AC}(20,20)$ & 0.0004 & 1.9190 & 1.1895 \\
\hline
\end{tabular}

Tabla 6.13: Errores relativos respecto a la minimización energética AC, AMBER

Queda patente la buena coincidencia entre los resultados de ambos procedimientos, con un error máximo relativo del $0.01 \%$ en el diámetro y del $2 \%$ en los momentos angulares.

\subsubsection{Desarrollo con potencial Morse}

La expresión (6.2) del potencial Morse reducida al sistema elemental de la figura6.3 adopta una expresión:

$$
U=-12 m D_{e}+2 m k_{\theta}\left(\Delta \theta_{2}\right)^{2}\left[1+k_{s}\left(\Delta \theta_{2}\right)^{4}\right]+4 m k_{\theta}\left(\Delta \theta_{1}\right)^{2}\left[1+k_{s}\left(\Delta \theta_{1}\right)^{4}\right]
$$

Aplicando la condición de mínimo 6.3 a 6.34 y empleando 6.30 se alcanza:

$$
-\Delta \theta_{2}\left[1+3 k_{s}\left(\Delta \theta_{2}\right)^{4}\right]+\Delta \theta_{1}\left[1+3 k_{s}\left(\Delta \theta_{1}\right)^{4}\right] \frac{C_{2 m} \cos \alpha}{\sqrt{1-\left(C_{2 m} \sin \alpha\right)^{2}}}=0
$$

donde $\Delta \theta_{1}, \Delta \theta_{2}$ están en función de $\alpha$ a través de (6.30), de forma que la ecuación (6.35) es implícita en $\alpha$. Resolviéndola numéricamente para $a_{0}=0.142 \mathrm{~nm}$ y sustituyendo en 6.25), obtenemos los valores de la tabla 6.14, que se presentan frente a los calculados mediante el mapeo conforme.

Comparando valores con los obtenidos para el potencial AMBER (tabla 6.10) se verifica que el potencial Morse no introduce ninguna diferencia en la obtención del diámetro inicial del nanotubo, al igual que ocurría en los nanotubos ZigZag.

Comparamos a continuación los esfuerzos obtenidos con los resultados correspondientes al modelo MSM. Los axiles siguen siendo nulos $\left(n_{0}^{a}=n_{0}^{b}=n_{0}^{c}=0\right)$ por hipótesis, y los momentos se calculan empleando la relación (6.18b). Resumimos en las tablas 6.15 y 6.16 los resultados obtenidos. 
Capítulo 6. Aproximación energética a la geometría inicial del nanotubo

\begin{tabular}{lcccc}
\hline & $\mathrm{m}$ & $D_{t}(\mathrm{~nm})[1.1)$ & $d_{0}(\mathrm{~nm}) \sqrt[6.25]{ }$ & $\alpha(\mathrm{rad})[6.35$ \\
\hline $\mathrm{AC}(3,3)$ & 3 & 0.4068 & 0.4185 & 0.5470 \\
$\mathrm{AC}(4,4)$ & 4 & 0.5424 & 0.5514 & 0.5375 \\
$\mathrm{AC}(5,5)$ & 5 & 0.6780 & 0.6853 & 0.5327 \\
$\mathrm{AC}(10,10)$ & 10 & 1.3560 & 1.3597 & 0.5260 \\
$\mathrm{AC}(15,15)$ & 15 & 2.0340 & 2.0365 & 0.5246 \\
$\mathrm{AC}(20,20)$ & 20 & 2.7120 & 2.7139 & 0.5242 \\
\hline
\end{tabular}

Tabla 6.14: Diámetros iniciales Armchair, Morse

\begin{tabular}{lccccc}
\hline & $n_{0}^{a}(\mathrm{nN})$ & $n_{0}^{b}(\mathrm{nN})$ & $n_{0}^{c}(\mathrm{nN})$ & $m_{0}^{1}(\mathrm{nNnm})$ & $m_{0}^{2}(\mathrm{nNnm})$ \\
\hline $\mathrm{AC}(3,3)$ & 0 & 0 & 0 & $5.2852 \cdot 10^{-2}$ & $4.2028 \cdot 10^{-2}$ \\
$\mathrm{AC}(4,4)$ & 0 & 0 & 0 & $2.9636 \cdot 10^{-2}$ & $2.5029 \cdot 10^{-2}$ \\
$\mathrm{AC}(5,5)$ & 0 & 0 & 0 & $1.8972 \cdot 10^{-2}$ & $1.6415 \cdot 10^{-2}$ \\
$\mathrm{AC}(10,10)$ & 0 & 0 & 0 & $4.7547 \cdot 10^{-3}$ & $4.2313 \cdot 10^{-3}$ \\
$\mathrm{AC}(15,15)$ & 0 & 0 & 0 & $2.1147 \cdot 10^{-3}$ & $1.8909 \cdot 10^{-3}$ \\
$\mathrm{AC}(20,20)$ & 0 & 0 & 0 & $1.1899 \cdot 10^{-3}$ & $1.0656 \cdot 10^{-3}$ \\
\hline
\end{tabular}

Tabla 6.15: Esfuerzos minimización energética AC, Morse

\begin{tabular}{lcccccc}
\hline & $d_{0}(\mathrm{~nm})$ & $n_{0}^{a}(\mathrm{nN})$ & $n_{0}^{b}(\mathrm{nN})$ & $n_{0}^{c}(\mathrm{nN})$ & $m_{0}^{1}(\mathrm{nNnm})$ & $m_{0}^{2}(\mathrm{nNnm})$ \\
\hline $\mathrm{AC}(3,3)$ & 0.4185 & $-1.13 \cdot 10^{-5}$ & $-1.47 \cdot 10^{-5}$ & $-1.13 \cdot 10^{-5}$ & $5.0672 \cdot 10^{-2}$ & $4.2167 \cdot 10^{-2}$ \\
$\mathrm{AC}(4,4)$ & 0.5514 & $-9.39 \cdot 10^{-6}$ & $5.42 \cdot 10^{-6}$ & $-9.39 \cdot 10^{-6}$ & $2.7779 \cdot 10^{-2}$ & $2.5062 \cdot 10^{-2}$ \\
$\mathrm{AC}(5,5)$ & 0.6853 & $2.35 \cdot 10^{-6}$ & $8.99 \cdot 10^{-6}$ & $2.35 \cdot 10^{-6}$ & $1.7549 \cdot 10^{-2}$ & $1.6416 \cdot 10^{-2}$ \\
$\mathrm{AC}(10,10)$ & 1.3597 & $4.95 \cdot 10^{-6}$ & $7.53 \cdot 10^{-6}$ & $4.95 \cdot 10^{-6}$ & $4.3214 \cdot 10^{-3}$ & $4.2182 \cdot 10^{-3}$ \\
$\mathrm{AC}(15,15)$ & 2.0365 & $7.27 \cdot 10^{-6}$ & $6.79 \cdot 10^{-6}$ & $7.27 \cdot 10^{-6}$ & $1.9228 \cdot 10^{-3}$ & $1.8792 \cdot 10^{-3}$ \\
$\mathrm{AC}(20,20)$ & 2.7139 & $4.69 \cdot 10^{-6}$ & $6.93 \cdot 10^{-6}$ & $4.69 \cdot 10^{-6}$ & $1.0922 \cdot 10^{-3}$ & $1.0519 \cdot 10^{-3}$ \\
\hline
\end{tabular}

Tabla 6.16: Esfuerzos modelo MSM (AC análisis lineal, Morse)

Para hacernos una idea de la correspondencia entre ambos procedimientos, se calculan los errores relativos para el diámetro y los momentos angulares tomando como solución exacta la minimización energética (los axiles del modelo MSM son despreciables):

\begin{tabular}{lccc}
\hline & $\varepsilon_{d}(\%)$ & $\varepsilon_{m^{1}}(\%)$ & $\varepsilon_{m^{2}}(\%)$ \\
\hline $\mathrm{AC}(3,3)$ & 0.0041 & 4.1243 & 0.3310 \\
$\mathrm{AC}(4,4)$ & 0.0011 & 6.2644 & 0.1342 \\
$\mathrm{AC}(5,5)$ & 0.0004 & 7.4995 & 0.0097 \\
$\mathrm{AC}(10,10)$ & 0.0004 & 9.1117 & 0.3091 \\
$\mathrm{AC}(15,15)$ & 0.0005 & 9.0749 & 0.6172 \\
$\mathrm{AC}(20,20)$ & 0.0004 & 8.2101 & 1.2885 \\
\hline
\end{tabular}

Tabla 6.17: Errores relativos respecto a la minimización energética AC, Morse

La concordancia de diámetros para SWNTs Armchair es bastante mejor que para los momentos angulares, con un error máximo relativo del $0.004 \%$ en el diámetro y un aceptable $9 \%$ en los momentos angulares. 


\subsection{Determinación del diámetro inicial para SWNTs Chiral}

En el caso de los nanotubos Chiral, las condiciones de simetría radial presentes en los ZigZag y Armchair desaparecen, complicando notablemente el proceso de obtención del diámetro inicial $d_{0}$.

Puesto que el diámetro inicial obtenido mediante el proceso minimizador con $a_{0}=$ cte. es mayor que el correspondiente al mapeo conforme, esto nos permite plantear un esquema de deformación a la lámina de grafeno plana equivalente a un aumento del diámetro, para aplicar finalmente cierto mapeo a la malla deformada.

Se definen en las figuras 6.5 y 6.6 las variables y notación empleadas en los desarrollos posteriores.

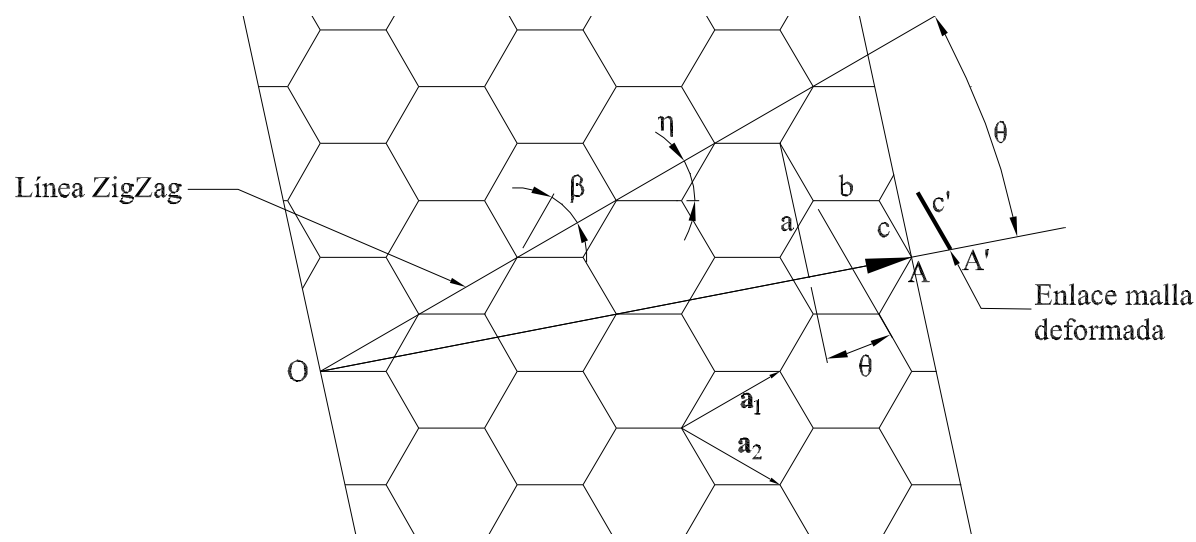

Figura 6.5: Orientaciones de enlace sistema elemental $\mathrm{CH}$

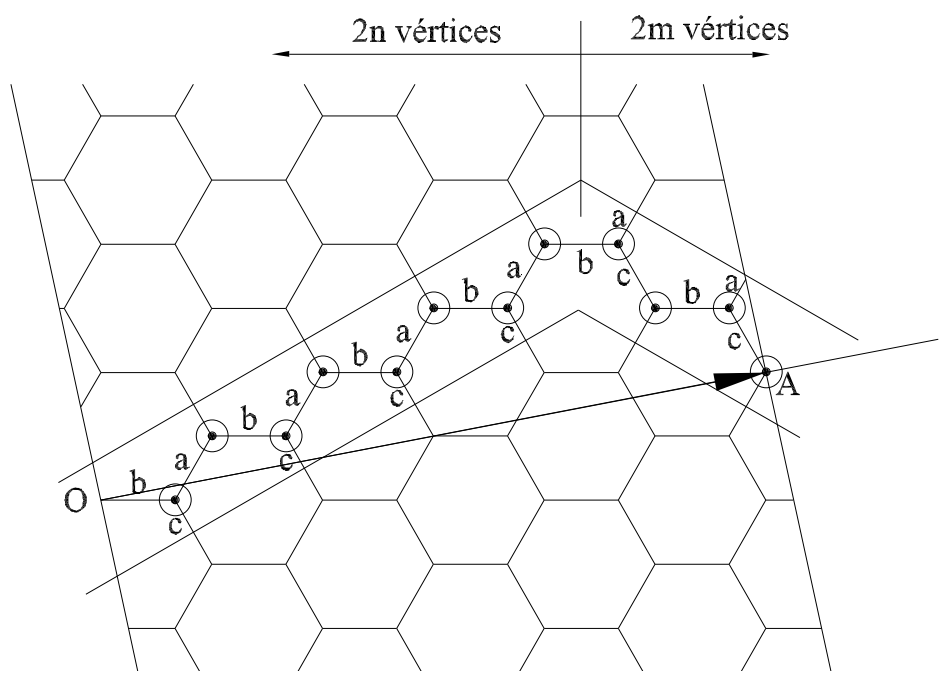

Figura 6.6: Notación enlaces sistema elemental $\mathrm{CH}$

Hipótesis sobre la geometría deformada de la malla:

1. La deformación planteada corresponde a una extensión de la malla en la dirección circunferencial OA del nanotubo 
2. Los enlaces tipo c (perpendiculares a la línea ZigZag) mantienen su orientación en el proceso de deformación

3. Adoptaremos el ángulo $\beta$ como parámetro independiente en la minimización energética

4. Todos los enlaces mantienen su longitud en el proceso de deformación (análogamente a los casos ZZ y AC).

5. Plantearemos el proceso de minimización energética sobre una banda unitaria como la de la figura 6.6, que se repite periódicamente en la dirección longitudinal del SWNT.

Teniendo en cuenta las hipótesis anteriores, esquematizamos en la figura 6.7 la deformación de una celda hexagonal tipo, producida por un desplazamiento impuesto $u$ en la dirección OA.

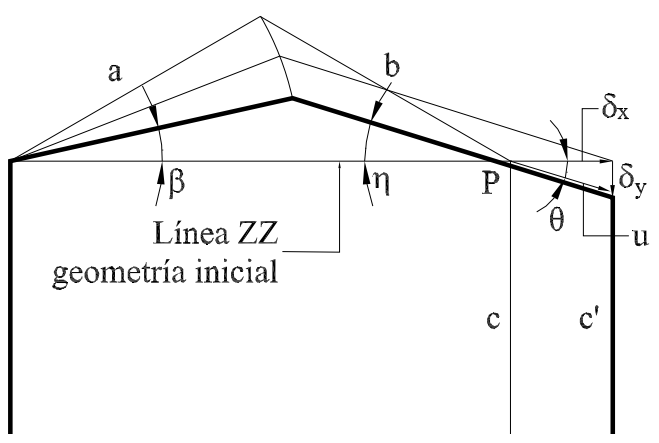

Figura 6.7: Esquema de deformación celda hexagonal tipo

Con el propósito de relacionar $\beta$ y $\eta$, establecemos la siguiente compatibilidad en $\mathrm{P}$ :

$$
\begin{aligned}
& a_{0} \cos \beta+a_{0} \cos \eta=2 a_{0} \frac{\sqrt{3}}{2}+\delta_{x} \\
& a_{0} \sin \beta-a_{0} \sin \eta=-\delta_{y}
\end{aligned}
$$

dividiendo por $a_{0}$ e introduciendo el desplazamiento $u$ :

$$
\begin{aligned}
& \cos \beta+\cos \eta=\sqrt{3}+\frac{u \cos \theta}{a_{0}} \\
& \sin \beta-\sin \eta=-\frac{u \sin \theta}{a_{0}}
\end{aligned}
$$

Multiplicando ahora (6.37a por $\sin \theta$, 6.37b por $\cos \theta$ y sumando podemos eliminar $u$ hasta llegar a:

$$
\sin (\theta+\beta)+\sin (\theta-\eta)=\sqrt{3} \sin \theta
$$

es decir

$$
\eta=\theta-\arcsin [\sqrt{3} \sin \theta-\sin (\theta+\beta)]
$$

Por otra parte, deberemos relacionar los ángulos entre enlaces con el ángulo $\beta$, valiéndonos de los esquemas representados en las figuras 6.8 y 6.9. En ellos se asume que el mapeo que convierte la malla distorsionada en la geometría inicial del nanotubo no modifica las orientaciones relativas de los enlaces respecto a las direcciones longitudinal y de la secante 1 al cilindro.

\footnotetext{
${ }^{1}$ resultado de proyectar el enlace AP (o bien BP) sobre la sección transversal del cilindro que envuelve idealmente al nanotubo
} 


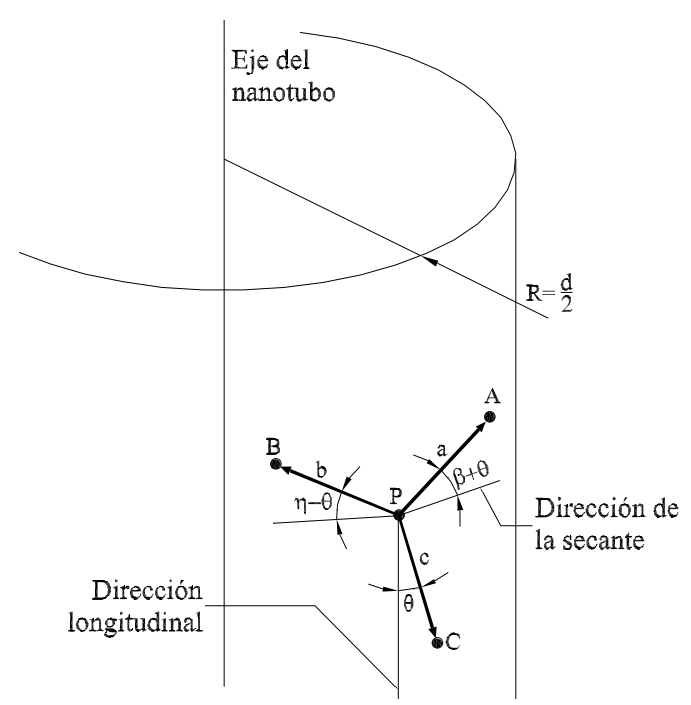

Figura 6.8: Esquema vectorial enlaces $\mathrm{CH}$, perspectiva

Proyectando los enlaces de la figura 6.8 sobre la sección transversal (figura 6.9) se debe verificar:

$$
\begin{aligned}
& d_{a}=a_{0} \cos (\theta+\beta) \\
& d_{b}=a_{0} \cos (\eta-\theta) \\
& d_{c}=a_{0} \sin \theta
\end{aligned}
$$

Asimismo, aplicando los teoremas del seno y del coseno sobre cada triángulo proyectado se llega a:

$$
\begin{aligned}
d_{i} & =d_{0} \sin \left(\frac{\varphi_{i}}{2}\right) \\
1-\frac{2 d_{i}^{2}}{d_{0}^{2}} & =\cos \varphi_{i} \quad i=a, b, c
\end{aligned}
$$

Introducimos los siguientes vectores auxiliares entre átomos:

$$
\begin{aligned}
& \mathbf{Z}_{1}=\overrightarrow{P A}=\left\{-d_{a} \sin \left(\frac{\varphi_{a}}{2}\right), d_{a} \cos \left(\frac{\varphi_{a}}{2}\right), a_{0} \sin (\theta+\beta)\right\}^{T} \\
& \mathbf{Z}_{2}=\overrightarrow{P B}=\left\{-d_{b} \sin \left(\frac{\varphi_{b}}{2}\right),-d_{b} \cos \left(\frac{\varphi_{b}}{2}\right), a_{0} \sin (\eta-\theta)\right\}^{T} \\
& \mathbf{Z}_{3}=\overrightarrow{P C}=\left\{-d_{c} \sin \left(\frac{\varphi_{c}}{2}\right), d_{c} \cos \left(\frac{\varphi_{c}}{2}\right),-a_{0} \sin \theta\right\}^{T}
\end{aligned}
$$

cuyos productos escalares nos permiten averiguar los ángulos buscados

$$
\begin{aligned}
& \mathbf{Z}_{1}^{T} \mathbf{Z}_{2}=a_{0}^{2} \cos \theta_{a b} \\
& \mathbf{Z}_{1}^{T} \mathbf{Z}_{3}=a_{0}^{2} \cos \theta_{a c} \\
& \mathbf{Z}_{2}^{T} \mathbf{Z}_{3}=a_{0}^{2} \cos \theta_{b c}
\end{aligned}
$$




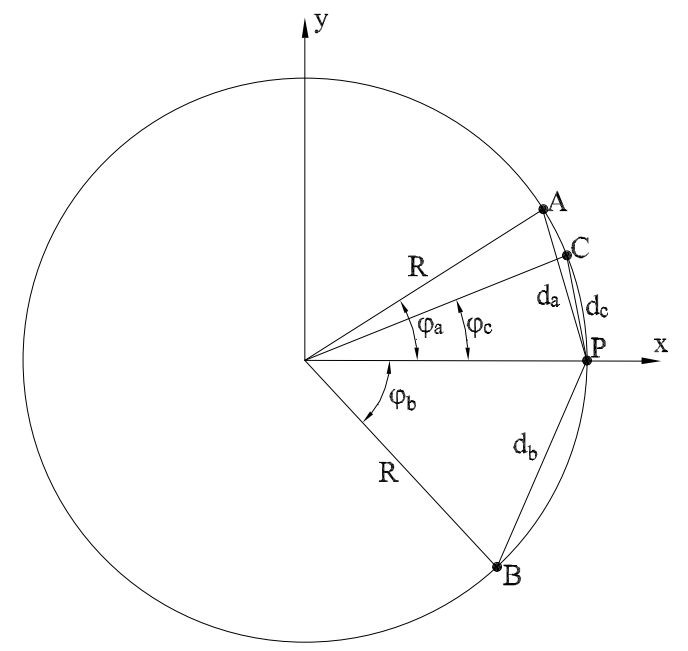

Figura 6.9: Esquema vectorial enlaces $\mathrm{CH}$, proyecciones en sección

Empleando las definiciones 6.42 en 6.43 y operando, podemos expresar:

$$
\begin{aligned}
& \cos \theta_{a b}=\left(\frac{a_{0}}{d_{0}}\right)^{2} \cos ^{2}(\theta+\beta) \cos ^{2}(\eta-\theta)- \\
& -\cos (\theta+\beta) \cos (\eta-\theta) \sqrt{1-\left(\frac{a_{0}}{d_{0}}\right)^{2} \cos ^{2}(\theta+\beta)} \sqrt{1-\left(\frac{a_{0}}{d_{0}}\right)^{2} \cos ^{2}(\eta-\theta)+} \\
& +\sin (\theta+\beta) \sin (\eta-\theta) \\
& +\sin \theta \cos (\theta+\beta) \sqrt{1-\left(\frac{a_{0}}{d_{0}}\right)^{2} \sin ^{2} \theta} \sqrt{1-\left(\frac{a_{0}}{d_{0}}\right)^{2} \cos ^{2}(\theta+\beta)-} \\
& -\cos \theta \sin (\theta+\beta) \\
& -\sin \theta \cos (\eta-\theta) \sqrt{1-\left(\frac{a_{0}}{d_{0}}\right)^{2} \sin ^{2} \theta} \sqrt{1-\left(\frac{a_{0}}{d_{0}}\right)^{2} \cos ^{2}(\eta-\theta)-} \\
& -\cos \theta \sin (\eta-\theta)
\end{aligned}
$$


Teniendo en cuenta la relación 6.39, podemos escribir 6.44) a 6.46) en función de $\beta$, por tanto las variaciones angulares serán:

$$
\begin{aligned}
\Delta \theta_{a b} & =\arccos \theta_{a b}(\beta)-\frac{2 \pi}{3} \\
\Delta \theta_{a c} & =\arccos \theta_{a c}(\beta)-\frac{2 \pi}{3} \\
\Delta \theta_{b c} & =\arccos \theta_{b c}(\beta)-\frac{2 \pi}{3}
\end{aligned}
$$

Sólo nos queda relacionar el diámetro $d_{0}$ con el parámetro independiente $\beta$. A partir de la figura 6.6, podemos proyectar enlaces en la dirección diametral OA al nanotubo, obteniendo para un $\mathrm{CH}(\mathrm{n}, \mathrm{m}){ }^{2}$ un esquema similar a $6.9 \mathrm{con}$ :

$$
\begin{array}{ll}
\mathrm{n} & \text { segmentos de longitud } d_{b} \\
\mathrm{n} & \text { segmentos de longitud } d_{a} \\
\mathrm{~m} & \text { segmentos de longitud } d_{b} \\
\mathrm{~m} & \text { segmentos de longitud } d_{c}
\end{array}
$$

Por tanto, para la circunferencia completa tenemos:

$$
\begin{aligned}
& n \varphi_{a}+(n+m) \varphi_{b}+m \varphi_{c}=2 \pi \\
& n \frac{\varphi_{a}}{2}+m \frac{\varphi_{c}}{2}=\pi-(n+m) \frac{\varphi_{b}}{2}
\end{aligned}
$$

tomando cosenos en 6.48 y reagrupando

$$
\cos \frac{n \varphi_{a}}{2} \cos \frac{m \varphi_{c}}{2}+\cos (n+m) \frac{\varphi_{b}}{2}=\sin \frac{n \varphi_{a}}{2} \sin \frac{m \varphi_{c}}{2}
$$

relación que podemos escribir en función de los polinomios de Chebyshev de primera clase como:

$$
T_{n}\left(\cos \frac{\varphi_{a}}{2}\right) T_{m}\left(\cos \frac{\varphi_{c}}{2}\right)+T_{n+m}\left(\cos \frac{\varphi_{b}}{2}\right)=\frac{T_{n}^{\prime}\left(\cos \frac{\varphi_{a}}{2}\right) T_{m}^{\prime}\left(\cos \frac{\varphi_{c}}{2}\right)}{n m} \sin \frac{\varphi_{a}}{2} \sin \frac{\varphi_{c}}{2}
$$

Nótese que la ecuación (6.50) relaciona los ángulos $\varphi_{i}$, que se pueden expresar en función de los segmentos de longitudes $d_{i}$ mediante 6.41). Éstos a su vez se pueden relacionar con las orientaciones angulares empleando (6.40), de forma que sustituyendo 6.39) sólo faltan dos parámetros por determinar $\beta$ y $d_{0}$. La condición de mínimo correspondiente a cada potencial nos proporcionará la ecuación adicional requerida para su determinación.

\subsubsection{Desarrollo con potencial AMBER}

Denotando por $n_{v}$ al número total de nudos (átomos) contenidos en la banda unitaria representada en la figura 6.6 es fácil comprobar que el número de ángulos $\theta_{a b}, \theta_{a c}, \theta_{b c}$ incluidos en la banda es el mismo para todos ellos e igual a:

$$
n_{v}=2 n+2 m
$$

Por tanto, la expresión del potencial AMBER (6.1) reducida a la banda unitaria considerada será:

$$
U=\frac{n_{v}}{2} k_{\theta}\left(\Delta \theta_{a b}^{2}+\Delta \theta_{a c}^{2}+\Delta \theta_{b c}^{2}\right)
$$

Derivando parcialmente 6.51) respecto al parámetro $\beta$ obtendremos las orientaciones de enlace que corresponden a la mínima energía potencial (equivalentemente a 6.3 ), es decir

$$
\Delta \theta_{a b} \frac{\partial \Delta \theta_{a b}}{\partial \beta}+\Delta \theta_{a c} \frac{\partial \Delta \theta_{a c}}{\partial \beta}+\Delta \theta_{b c} \frac{\partial \Delta \theta_{b c}}{\partial \beta}=0
$$

\footnotetext{
${ }^{2}$ En la figura 6.6 se ha representado como ejemplo un $\mathrm{CH}(4,2)$
} 
Sustituyendo 6.47), la ecuación 6.52) es implícita en $\beta$ y $d_{0}$. Para su obtención diseñamos el proceso iterativo esquematizado en la figura 6.10. Adicionalmente conviene añadir los siguientes comentarios:

1. La solución numérica por el método de Newton requiere una buena aproximación inicial, que en cada caso será la solución de la iteración anterior.

2. En particular, para la primera iteración se adopta como hipótesis inicial el diámetro $d_{0}^{0}$ definido para el mapeo conforme y $\beta^{0}=\pi / 6$ correspondiente a la lámina de grafeno no distorsionada.

3. El error máximo adoptado para aceptar la convergencia del proceso es $\varepsilon=10^{-7}$ y para los métodos de Newton $\varepsilon=10^{-6}$.

Los resultados obtenidos para distintos valores de los enteros $(n, m)$ en comparación con los diámetros correspondientes al mapeo conforme se resumen en la tabla 6.18 .

\begin{tabular}{lccccc}
\hline & $\mathrm{n}$ & $\mathrm{m}$ & $D_{t}(\mathrm{~nm})(1.1)$ & $d_{0}(\mathrm{~nm})(6.50)$ & $\beta(\mathrm{rad})(6.52)$ \\
\hline $\mathrm{CH}(4,2)$ & 4 & 2 & 0.4143 & 0.4267 & 0.4864 \\
$\mathrm{CH}(5,3)$ & 5 & 3 & 0.5480 & 0.5568 & 0.5037 \\
$\mathrm{CH}(6,3)$ & 6 & 3 & 0.6214 & 0.6299 & 0.5061 \\
$\mathrm{CH}(6,4)$ & 6 & 4 & 0.6825 & 0.6892 & 0.5116 \\
$\mathrm{CH}(7,4)$ & 7 & 4 & 0.7550 & 0.7616 & 0.5125 \\
$\mathrm{CH}(8,4)$ & 8 & 4 & 0.8285 & 0.8350 & 0.5136 \\
\hline
\end{tabular}

Tabla 6.18: Diámetros iniciales Chiral, AMBER

Se comparan también en las tablas 6.19 y 6.20 los esfuerzos obtenidos en el proceso minimizador (axiles nulos por hipótesis y momentos mediante (6.15) ) con los extraídos del modelo MSM en análisis lineal, todos ellos en ausencia de cargas externas.

\begin{tabular}{lcccccc}
\hline & $n_{0}^{a}(\mathrm{nN})$ & $n_{0}^{b}(\mathrm{nN})$ & $n_{0}^{c}(\mathrm{nN})$ & $m_{0}^{a b}(\mathrm{nNnm})$ & $m_{0}^{a c}(\mathrm{nNnm})$ & $m_{0}^{b c}(\mathrm{nNnm})$ \\
\hline $\mathrm{CH}(4,2)$ & 0 & 0 & 0 & $5.0826 \cdot 10^{-2}$ & $3.0955 \cdot 10^{-2}$ & $5.2991 \cdot 10^{-2}$ \\
$\mathrm{CH}(5,3)$ & 0 & 0 & 0 & $2.6422 \cdot 10^{-2}$ & $1.6852 \cdot 10^{-2}$ & $3.3300 \cdot 10^{-2}$ \\
$\mathrm{CH}(6,3)$ & 0 & 0 & 0 & $2.0642 \cdot 10^{-2}$ & $1.4645 \cdot 10^{-2}$ & $2.4450 \cdot 10^{-2}$ \\
$\mathrm{CH}(6,4)$ & 0 & 0 & 0 & $1.6230 \cdot 10^{-2}$ & $1.0323 \cdot 10^{-2}$ & $2.2712 \cdot 10^{-2}$ \\
$\mathrm{CH}(7,4)$ & 0 & 0 & 0 & $1.3443 \cdot 10^{-2}$ & $9.3869 \cdot 10^{-3}$ & $1.7514 \cdot 10^{-2}$ \\
$\mathrm{CH}(8,4)$ & 0 & 0 & 0 & $1.1238 \cdot 10^{-2}$ & $8.3822 \cdot 10^{-3}$ & $1.3914 \cdot 10^{-2}$ \\
\hline
\end{tabular}

Tabla 6.19: Esfuerzos minimización energética $C H, A M B E R$

\begin{tabular}{cccccccc}
\hline & $d_{0}(\mathrm{~nm})$ & $n_{0}^{a}(\mathrm{nN})$ & $n_{0}^{b}(\mathrm{nN})$ & $n_{0}^{c}(\mathrm{nN})$ & $m_{0}^{a b}(\mathrm{nNnm})$ & $m_{0}^{a c}(\mathrm{nNnm})$ & $m_{0}^{b c}(\mathrm{nNnm})$ \\
\hline $\mathrm{CH}(4,2)$ & 0.4279 & $1.64 \cdot 10^{-2}$ & $1.30 \cdot 10^{-3}$ & $5.02 \cdot 10^{-3}$ & $4.8727 \cdot 10^{-2}$ & $3.9964 \cdot 10^{-2}$ & $4.6415 \cdot 10^{-2}$ \\
$\mathrm{CH}(5,3)$ & 0.5567 & $-1.88 \cdot 10^{-2}$ & $5.78 \cdot 10^{-4}$ & $-9.71 \cdot 10^{-3}$ & $2.7315 \cdot 10^{-2}$ & $2.5751 \cdot 10^{-2}$ & $2.6152 \cdot 10^{-2}$ \\
$\mathrm{CH}(6,3)$ & 0.6295 & $8.78 \cdot 10^{-5}$ & $-1.13 \cdot 10^{-4}$ & $2.84 \cdot 10^{-4}$ & $2.0784 \cdot 10^{-2}$ & $1.9021 \cdot 10^{-2}$ & $2.0159 \cdot 10^{-2}$ \\
$\mathrm{CH}(6,4)$ & 0.6888 & $-8.70 \cdot 10^{-3}$ & $-2.20 \cdot 10^{-5}$ & $-9.06 \cdot 10^{-3}$ & $1.7066 \cdot 10^{-2}$ & $1.6231 \cdot 10^{-2}$ & $1.6702 \cdot 10^{-2}$ \\
$\mathrm{CH}(7,4)$ & 0.7616 & $-1.16 \cdot 10^{-3}$ & $4.72 \cdot 10^{-5}$ & $-2.00 \cdot 10^{-3}$ & $1.3804 \cdot 10^{-2}$ & $1.3096 \cdot 10^{-2}$ & $1.3608 \cdot 10^{-2}$ \\
$\mathrm{CH}(8,4)$ & 0.8355 & $-5.00 \cdot 10^{-5}$ & $1.24 \cdot 10^{-5}$ & $-2.06 \cdot 10^{-5}$ & $1.1445 \cdot 10^{-2}$ & $1.0891 \cdot 10^{-2}$ & $1.1259 \cdot 10^{-2}$ \\
\hline
\end{tabular}

Tabla 6.20: Esfuerzos modelo MSM (CH análisis lineal, AMBER)

Podemos observar que los axiles obtenidos con el modelo MSM son muy reducidos, aunque son superiores a los calculados en los casos ZZ y AC. Tomando como solución de referencia la corres- 


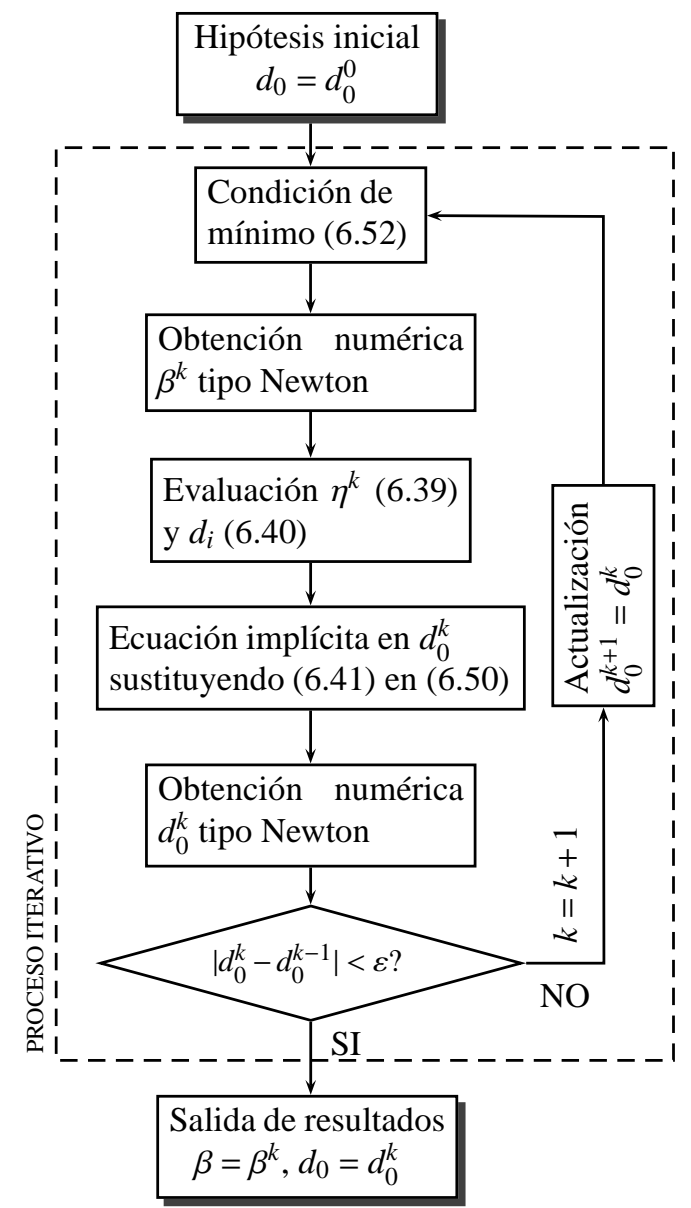

Figura 6.10: Diagrama de flujo del proceso iterativo para la obtención de $d_{0}$

pondiente al proceso minimizador, resumimos a continuación los errores relativos para el diámetro y los momentos angulares:

\begin{tabular}{lcccc}
\hline & $\varepsilon_{d}(\%)$ & $\varepsilon_{m^{a b}}(\%)$ & $\varepsilon_{m^{a c}}(\%)$ & $\varepsilon_{m^{b c}}(\%)$ \\
\hline $\mathrm{CH}(4,2)$ & 0.2885 & 4.1290 & 29.1021 & 12.4095 \\
$\mathrm{CH}(5,3)$ & 0.0167 & 3.3801 & 52.8062 & 21.4649 \\
$\mathrm{CH}(6,3)$ & 0.0729 & 0.6879 & 29.8796 & 17.5506 \\
$\mathrm{CH}(6,4)$ & 0.0638 & 5.1535 & 57.2205 & 26.4636 \\
$\mathrm{CH}(7,4)$ & 0.0070 & 2.6801 & 39.5101 & 22.3059 \\
$\mathrm{CH}(8,4)$ & 0.0578 & 1.8410 & 29.9340 & 19.0874 \\
\hline
\end{tabular}

Tabla 6.21: Errores relativos respecto a la minimización energética $C H, A M B E R$

Podemos comprobar la extremada coincidencia entre los resultados de ambos procedimientos en lo relativo al diámetro inicial en ausencia de cargas externas, con un error máximo relativo del $0.29 \%$. Sin embargo, los momentos angulares presentan un error relativo máximo del $57 \%$, produciendo diferencias muy superiores a sus análogas en los casos ZZ y AC. Éste efecto puede ser debido a la falta de simetría radial de estos nanotubos, donde la hipótesis de geometría inicial cilíndrica puede alejarse de la situación real. Además, la supuesta conservación de orientaciones en el mapeo adoptado 
puede ser cuestionable, sobre todo en nanotubos $\mathrm{CH}$ de reducido diámetro.

A pesar de las diferencias observadas, el procedimiento minimizador constituye una mejora importante hacia la determinación teórica de la geometría inicial de los SWNTs.

\subsubsection{Desarrollo con potencial Morse}

Conviene recordar aquí que tenemos $n_{v}$ ángulos de cada tipo $\theta_{a b}, \theta_{a c}, \theta_{b c}$ incluidos en la banda elemental representada en la figura 6.6 de forma que la expresión (6.2) del potencial Morse reducida a dicha banda se puede escribir:

$$
U=-\frac{3 n_{v}}{2} D_{e}+\frac{n_{v} k_{\theta}}{2}\left\{\left(\Delta \theta_{a b}\right)^{2}\left[1+k_{s}\left(\Delta \theta_{a b}\right)^{4}\right]+\left(\Delta \theta_{a c}\right)^{2}\left[1+k_{s}\left(\Delta \theta_{a c}\right)^{4}\right]+\left(\Delta \theta_{b c}\right)^{2}\left[1+k_{s}\left(\Delta \theta_{b c}\right)^{4}\right]\right\}
$$

Derivando parcialmente (6.53) respecto a $\beta$ obtendremos las orientaciones de enlace que corresponden al potencial mínimo, tal como se ha mostrado para el potencial AMBER.

$$
\Delta \theta_{a b}\left[1+3 k_{s}\left(\Delta \theta_{a b}\right)^{4}\right] \frac{\partial \Delta \theta_{a b}}{\partial \beta}+\Delta \theta_{a c}\left[1+3 k_{s}\left(\Delta \theta_{a c}\right)^{4}\right] \frac{\partial \Delta \theta_{a c}}{\partial \beta}+\Delta \theta_{b c}\left[1+3 k_{s}\left(\Delta \theta_{b c}\right)^{4}\right] \frac{\partial \Delta \theta_{b c}}{\partial \beta}=0
$$

Sustituyendo 6.47), la ecuación (6.54) es implícita en $\beta$ y $d_{0}$. La determinación de ambos valores se realiza en este caso mediante un proceso iterativo análogo al representado en la figura 6.10, aunque reemplazando la condición de mínimo 6.52) por 6.54). Los resultados obtenidos se comparan en la tabla 6.22 con los diámetros asociados al mapeo conforme.

\begin{tabular}{lccccc}
\hline & $\mathrm{n}$ & $\mathrm{m}$ & $D_{t}(\mathrm{~nm})(1.1)$ & $d_{0}(\mathrm{~nm})(6.50)$ & $\beta(\mathrm{rad})(6.52)$ \\
\hline $\mathrm{CH}(4,2)$ & 4 & 2 & 0.4143 & 0.4267 & 0.4864 \\
$\mathrm{CH}(5,3)$ & 5 & 3 & 0.5480 & 0.5568 & 0.5037 \\
$\mathrm{CH}(6,3)$ & 6 & 3 & 0.6214 & 0.6299 & 0.5061 \\
$\mathrm{CH}(6,4)$ & 6 & 4 & 0.6825 & 0.6892 & 0.5116 \\
$\mathrm{CH}(7,4)$ & 7 & 4 & 0.7550 & 0.7616 & 0.5125 \\
$\mathrm{CH}(8,4)$ & 8 & 4 & 0.8285 & 0.8350 & 0.5136 \\
\hline
\end{tabular}

Tabla 6.22: Diámetros iniciales Chiral, Morse

Una vez más, comparando estos valores con los de la tabla 6.18 se concluye que el potencial adoptado no influye significativamente en la determinación del diámetro inicial en ausencia de cargas externas.

Se incluyen en las tablas 6.23 y 6.24 los esfuerzos obtenidos tanto en el proceso minimizador como con el modelo MSM (análisis lineal).

\begin{tabular}{lcccccc}
\hline & $n_{0}^{a}(\mathrm{nN})$ & $n_{0}^{b}(\mathrm{nN})$ & $n_{0}^{c}(\mathrm{nN})$ & $m_{0}^{a b}(\mathrm{nNnm})$ & $m_{0}^{a c}(\mathrm{nNnm})$ & $m_{0}^{b c}(\mathrm{nNnm})$ \\
\hline $\mathrm{CH}(4,2)$ & 0 & 0 & 0 & $5.2208 \cdot 10^{-2}$ & $3.1797 \cdot 10^{-2}$ & $5.4433 \cdot 10^{-2}$ \\
$\mathrm{CH}(5,3)$ & 0 & 0 & 0 & $2.7140 \cdot 10^{-2}$ & $1.7310 \cdot 10^{-2}$ & $3.4205 \cdot 10^{-2}$ \\
$\mathrm{CH}(6,3)$ & 0 & 0 & 0 & $2.1203 \cdot 10^{-2}$ & $1.5043 \cdot 10^{-2}$ & $2.5115 \cdot 10^{-2}$ \\
$\mathrm{CH}(6,4)$ & 0 & 0 & 0 & $1.6671 \cdot 10^{-2}$ & $1.0604 \cdot 10^{-2}$ & $2.3329 \cdot 10^{-2}$ \\
$\mathrm{CH}(7,4)$ & 0 & 0 & 0 & $1.3809 \cdot 10^{-2}$ & $9.6419 \cdot 10^{-3}$ & $1.7990 \cdot 10^{-2}$ \\
$\mathrm{CH}(8,4)$ & 0 & 0 & 0 & $1.1544 \cdot 10^{-2}$ & $8.6099 \cdot 10^{-3}$ & $1.4292 \cdot 10^{-2}$ \\
\hline
\end{tabular}

Tabla 6.23: Esfuerzos minimización energética $\mathrm{CH}$, Morse

Tomando como solución de referencia la correspondiente al proceso minimizador, resumimos a continuación los errores relativos para el diámetro y los momentos angulares: 
6.5. Consideraciones sobre la coherencia de la formulación

\begin{tabular}{cccccccc}
\hline & $d_{0}(\mathrm{~nm})$ & $n_{0}^{a}(\mathrm{nN})$ & $n_{0}^{b}(\mathrm{nN})$ & $n_{0}^{c}(\mathrm{nN})$ & $m_{0}^{a b}(\mathrm{nNnm})$ & $m_{0}^{a c}(\mathrm{nNnm})$ & $m_{0}^{b c}(\mathrm{nNnm})$ \\
\hline $\mathrm{CH}(4,2)$ & 0.4266 & $1.95 \cdot 10^{-2}$ & $1.70 \cdot 10^{-3}$ & $5.89 \cdot 10^{-3}$ & $5.0162 \cdot 10^{-2}$ & $4.1129 \cdot 10^{-2}$ & $4.7909 \cdot 10^{-2}$ \\
$\mathrm{CH}(5,3)$ & 0.5563 & $-5.38 \cdot 10^{-3}$ & $2.24 \cdot 10^{-4}$ & $-1.15 \cdot 10^{-3}$ & $2.7739 \cdot 10^{-2}$ & $2.4920 \cdot 10^{-2}$ & $2.6691 \cdot 10^{-2}$ \\
$\mathrm{CH}(6,3)$ & 0.6300 & $-2.14 \cdot 10^{-4}$ & $3.62 \cdot 10^{-5}$ & $-2.08 \cdot 10^{-5}$ & $2.1345 \cdot 10^{-2}$ & $1.9551 \cdot 10^{-2}$ & $2.0701 \cdot 10^{-2}$ \\
$\mathrm{CH}(6,4)$ & 0.6896 & $-7.72 \cdot 10^{-3}$ & $1.48 \cdot 10^{-4}$ & $-7.52 \cdot 10^{-3}$ & $1.7501 \cdot 10^{-2}$ & $1.6598 \cdot 10^{-2}$ & $1.7132 \cdot 10^{-2}$ \\
$\mathrm{CH}(7,4)$ & 0.7620 & $-1.15 \cdot 10^{-3}$ & $1.06 \cdot 10^{-4}$ & $-1.36 \cdot 10^{-3}$ & $1.4188 \cdot 10^{-2}$ & $1.3438 \cdot 10^{-2}$ & $1.3957 \cdot 10^{-2}$ \\
$\mathrm{CH}(8,4)$ & 0.8353 & $-8.02 \cdot 10^{-5}$ & $1.30 \cdot 10^{-5}$ & $-3.46 \cdot 10^{-5}$ & $1.1760 \cdot 10^{-2}$ & $1.1191 \cdot 10^{-2}$ & $1.1567 \cdot 10^{-2}$ \\
\hline
\end{tabular}

Tabla 6.24: Esfuerzos modelo MSM (CH análisis lineal, Morse)

\begin{tabular}{lcccc}
\hline & $\varepsilon_{d}(\%)$ & $\varepsilon_{m^{a b}}(\%)$ & $\varepsilon_{m^{a c}}(\%)$ & $\varepsilon_{m^{b c}}(\%)$ \\
\hline $\mathrm{CH}(4,2)$ & 0.0239 & 3.9178 & 29.3491 & 11.9846 \\
$\mathrm{CH}(5,3)$ & 0.0864 & 2.2067 & 43.9664 & 21.9674 \\
$\mathrm{CH}(6,3)$ & 0.0157 & 0.6702 & 29.9681 & 17.5754 \\
$\mathrm{CH}(6,4)$ & 0.0585 & 4.9807 & 56.5292 & 26.5643 \\
$\mathrm{CH}(7,4)$ & 0.0596 & 2.7447 & 39.3753 & 22.4212 \\
$\mathrm{CH}(8,4)$ & 0.0339 & 1.8738 & 29.9768 & 19.0675 \\
\hline
\end{tabular}

Tabla 6.25: Errores relativos respecto a la minimización energética $\mathrm{CH}$, Morse

Por tanto, se pueden extender aquí las conclusiones alcanzadas para el potencial AMBER respecto a la íntima correspondencia en diámetros $\left(\varepsilon_{d, \max }=0.06 \%\right)$ y el importante sesgo en momentos angulares (error máximo relativo $\simeq 57 \%$ ).

\subsection{Consideraciones sobre la coherencia de la formulación}

Incluimos en esta sección algunas comprobaciones adicionales que permiten establecer la equivalencia entre la formulación del caso más general para los SWNTs Chiral y aquellas desarrolladas simplificadamente para los nanotubos ZigZag (sección 6.2) y Armchair (sección 6.3).

\subsubsection{Comprobación Chiral-ZigZag}

Los nanotubos ZZ(n,0) se caracterizan por una orientación del vector Quiral $\theta=0$ y por el segundo entero $m=0$ en la base generadora de la malla hexagonal. Anulando $\theta$ en (6.38) se llega a:

$$
\begin{aligned}
\sin \eta & =\sin \beta \\
\eta & =\beta
\end{aligned}
$$

Sustituyendo 6.5.1) en las expresiones 6.44) a 6.46 y operando:

$$
\begin{aligned}
& \cos \theta_{a b}=2\left(\frac{a_{0}}{d_{0}}\right)^{2} \cos ^{4} \beta-\cos ^{2} \beta+\sin ^{2} \beta \\
& \cos \theta_{a c}=-\sin \beta \\
& \cos \theta_{b c}=-\sin \eta
\end{aligned}
$$

Como cabía esperar, resulta $\theta_{a c}=\theta_{b c}=\theta_{1}$ de forma consistente con la figura 6.1 Por otra parte, teniendo en cuenta las definiciones de $\alpha, \beta$ y $\eta$, es inmediato que

$$
\alpha=\beta=\eta
$$


luego, de 6.5 se sigue:

$$
\frac{a_{0}}{d_{0}} \cos \beta=\sin \left(\frac{\pi}{2 n}\right)
$$

Sustituyendo 6.57 en 6.56a y operando se llega a:

$$
\cos \theta_{a b}=\sin ^{2} \beta\left(1+\cos \frac{\pi}{n}\right)-\cos \frac{\pi}{n}
$$

expresión completamente equivalente a (6.9), luego $\theta_{a b}=\theta_{2}$.

Para relacionar ahora el diámetro $\operatorname{con} \beta$, forzamos $d_{a}=d_{b}$ y $d_{c}=0$ (figura 6.9) en (6.41a), es decir

$$
\varphi_{a}=\varphi_{b} \quad \varphi_{c}=0
$$

que junto con $m=0$, reducen 6.48 a:

$$
\frac{\varphi_{a}}{2}=\frac{\pi}{2 n}
$$

Tomando senos en 6.60) y empleando 6.57):

$$
\sin \frac{\varphi_{a}}{2}=\sin \left(\frac{\pi}{2 n}\right)=\frac{a_{0}}{d_{0}} \cos \beta
$$

con lo que se llega a:

$$
d_{0}=\frac{a_{0} \cos \beta}{\sin \left(\frac{\pi}{2 n}\right)}
$$

formalmente idéntica a 6.5).

En consecuencia, se ha mostrado que la formulación general para los nanotubos $\mathrm{CH}$ particularizada para el caso ZZ es completamente equivalente a la desarrollada en la sección 6.2. En la tabla 6.26 extraemos algunos valores mediante ambos procedimientos de cálculo para el potencial AMBER, así como los errores relativos tomando como referencia la formulación directa para los ZZ.

\begin{tabular}{lccccc}
\hline & & \multicolumn{3}{c}{ ZZ AMBER } & CH AMBER \\
& $\mathrm{n}$ & $D_{t}(\mathrm{~nm})(1.1)$ & $d_{0}(\mathrm{~nm})$ & $d_{0}(\mathrm{~nm})$ & $\varepsilon_{d}(\%)$ \\
\hline $\mathrm{ZZ}(4,0)$ & 4 & 0.3132 & 0.3356 & 0.3345 & 0.3504 \\
$\mathrm{ZZ}(5,0)$ & 5 & 0.3914 & 0.4100 & 0.4095 & 0.1332 \\
$\mathrm{ZZ}(6,0)$ & 6 & 0.4697 & 0.4854 & 0.4851 & 0.0610 \\
$\mathrm{ZZ}(10,0)$ & 10 & 0.7829 & 0.7925 & 0.7924 & 0.0072 \\
$\mathrm{ZZ}(15,0)$ & 15 & 1.1743 & 1.1807 & 1.1807 & 0.0017 \\
$\mathrm{ZZ}(20,0)$ & 20 & 1.5658 & 1.5706 & 1.5706 & 0.0000 \\
\hline
\end{tabular}

Tabla 6.26: Comparación diámetros obtenidos Chiral-ZigZag, AMBER

También se efectuó la comparación para el potencial Morse, aunque los valores y errores obtenidos fueron prácticamente iguales a los de la tabla 6.26 .

Los pequeños errores producidos pueden asociarse a la precisión adoptada, tanto en el proceso iterativo como en los métodos de Newton empleados para los SWNTs Chiral, cuyos ratios de error se acumulan en los valores finales.

\subsubsection{Comprobación Chiral-Armchair}

En la figura 6.11 se representa una superposición gráfica de los parámetros de orientación definidos para las formulaciones Chiral y Armchair sobre la malla hexagonal. 


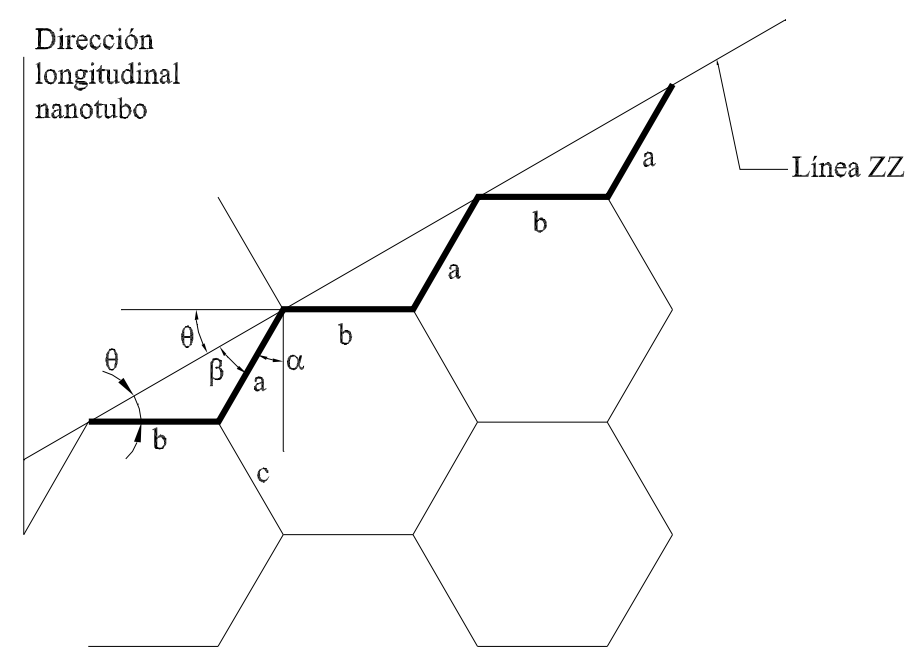

Figura 6.11: Definición de parámetros formulaciones Chiral y Armchair

En general, un SWNT Armchair se caracteriza por $n=m$, además de verificar las siguientes relaciones angulares:

$$
\begin{aligned}
& \eta=\theta \\
& \beta+\theta=\frac{\pi}{2}-\alpha
\end{aligned}
$$

que sustituidas en 6.38) llevan a:

$$
\cos \alpha=\sqrt{3} \sin \theta
$$

de hecho, (6.40) se convierte en:

$$
\begin{aligned}
& d_{a}=a_{0} \sin \alpha \\
& d_{b}=a_{0} \\
& d_{c}=\frac{a_{0} \cos \alpha}{\sqrt{3}}
\end{aligned}
$$

Una rápida inspección a la figura 6.9 transformada en la sección transversal de un nanotubo AC, debería permitir establecer la condición de simetría radial $d_{a}=d_{c}$. Sin embargo, partiendo de la formulación $\mathrm{CH}$ esta condición no se verifica en general. La única manera de cumplirla en (6.65) consiste en forzar $\alpha=\pi / 6$, valor que sólo sería posible mediante el mapeo conforme y que contradice claramente los resultados de la formulación.

En consecuencia, ambas formulaciones no son idénticas en relación a las orientaciones finales de los enlaces en la malla distorsionada y mapeada sobre el cilindro. En concreto, la formulación para los SWNTs Armchair mantiene la orientación de los enlaces b fija en la dirección transversal al nanotubo, y la formulación general para los Chiral mantiene la orientación de los enlaces c perpendicular a la línea ZZ. Si particularizamos la distorsión planteada para la malla en el caso $\mathrm{CH}$ al caso $\mathrm{AC}$, dos filas consecutivas de enlaces $\mathrm{b}$ (perpendiculares al eje del nanotubo) quedarán decalados un cierto valor en la dirección de eje del tubo.

A pesar de ello, proyectando los enlaces a y b (en trazo grueso en la figura 6.11) sobre la dirección transversal al nanotubo hasta completar la circunferencia, podemos escribir:

$$
2 n \varphi_{a}+2 n \varphi_{b}=2 \pi
$$


que es formalmente idéntica a la relación que se obtendría forzando $n=m$ y $\varphi_{a}=\varphi_{c}$ (simetría radial) en (6.48). Dividiendo por $4 n$ y tomando razones trigonométricas:

$$
\begin{aligned}
& \sin \left(\frac{\varphi_{a}}{2}+\frac{\varphi_{b}}{2}\right)=\sin \frac{\varphi_{a}}{2} \cos \frac{\varphi_{b}}{2}+\sin \frac{\varphi_{b}}{2} \cos \frac{\varphi_{a}}{2}=\sin \frac{\pi}{2 n} \\
& \cos \left(\frac{\varphi_{a}}{2}+\frac{\varphi_{b}}{2}\right)=\cos \frac{\varphi_{a}}{2} \cos \frac{\varphi_{b}}{2}-\sin \frac{\varphi_{b}}{2} \sin \frac{\varphi_{a}}{2}=\cos \frac{\pi}{2 n}
\end{aligned}
$$

Elevando 6.67) al cuadrado y sustituyendo 6.68), se llega a:

$$
\sin ^{2} \frac{\varphi_{a}}{2} \cos ^{2} \frac{\varphi_{b}}{2}+\cos ^{2} \frac{\varphi_{a}}{2} \sin ^{2} \frac{\varphi_{b}}{2}+2 \sin ^{2} \frac{\varphi_{a}}{2} \sin ^{2} \frac{\varphi_{b}}{2}+2 \sin \frac{\varphi_{a}}{2} \sin \frac{\varphi_{b}}{2} \cos \frac{\pi}{2 n}=\sin ^{2} \frac{\pi}{2 n}
$$

Sustituyendo ahora 6.65a y 6.65b) en 6.41), tenemos:

$$
\begin{aligned}
& \sin \frac{\varphi_{a}}{2}=\frac{a_{0} \sin \alpha}{d_{0}} \\
& \sin \frac{\varphi_{b}}{2}=\frac{a_{0}}{d_{0}}
\end{aligned}
$$

empleando adecuadamente (6.70) en 6.69) junto con (6.24) y operando, obtenemos:

$$
d_{0}=\frac{a_{0}}{S_{2 n}} \sqrt{1+\sin ^{2} \alpha+2 \sin \alpha C_{2 n}}
$$

formalmente idéntica a 6.25). Por tanto, a pesar de las diferencias presentes en las orientaciones, el diámetro obtenido será el mismo por ambos procedimientos. Para comprobarlo, extraemos en la tabla 6.27 algunos valores del diámetro obtenido por ambos procedimientos de cálculo empleando el potencial AMBER, así como el error relativo producido tomando como referencia la formulación directa para los AC.

\begin{tabular}{lccccc}
\hline & & & AC AMBER & CH AMBER & \\
& $\mathrm{n}=\mathrm{m}$ & $D_{t}(\mathrm{~nm})(1.1)$ & $d_{0}(\mathrm{~nm})$ & $d_{0}(\mathrm{~nm})$ & $\varepsilon_{d}(\%)$ \\
\hline $\mathrm{AC}(3,3)$ & 3 & 0.4068 & 0.4185 & 0.4154 & 0.7407 \\
$\mathrm{AC}(4,4)$ & 4 & 0.5424 & 0.5514 & 0.5489 & 0.4534 \\
$\mathrm{AC}(5,5)$ & 5 & 0.6780 & 0.6853 & 0.6833 & 0.2918 \\
$\mathrm{AC}(10,10)$ & 10 & 1.3560 & 1.3597 & 1.3586 & 0.0809 \\
$\mathrm{AC}(15,15)$ & 15 & 2.0340 & 2.0365 & 2.0358 & 0.0344 \\
$\mathrm{AC}(20,20)$ & 20 & 2.7120 & 2.7139 & 2.7189 & 0.1842 \\
\hline
\end{tabular}

Tabla 6.27: Comparación diámetros obtenidos Chiral-Armchair, AMBER

También se efectuó la comparación para el potencial Morse, aunque los valores y errores obtenidos fueron prácticamente iguales a los de la tabla 6.27. Al igual que en la comparación con los SWNTs ZigZag, los pequeños errores producidos pueden asociarse a la precisión adoptada en el proceso numérico de cálculo del diámetro para los SWNTs Chiral.

Comentario 6.5.1 Como se ha mostrado en esta sección, diferentes mapeos y distorsiones de la malla hexagonal pueden producir los mismos diámetros obtenidos en ausencia de cargas externas. El presente capítulo queda limitado a la obtención del diámetro inicial con mayor precisión que el correspondiente al mapeo conforme, ampliamente empleado en la literatura. La determinación teórica de un mapeo físicamente razonable que transforme la lámina de grafeno en la nueva geometría inicial constituye un reto fuera de los objetivos de esta tesis. 


\section{Capítulo 7}

\section{Conclusiones y líneas de investigación propuestas}

\subsection{Resumen del trabajo realizado}

Esta tesis doctoral tiene por objeto la formulación general del modelo MSM stick-spiral y su aplicación a los nanotubos de carbono monocapa. Se ha explorado dicha formulación y sus resultados tanto en el marco del análisis geométricamente lineal como en el no lineal, además de contrastar las posibles diferencias en la respuesta estructural de los SWNTs debido a la consideración de dos potenciales interatómicos (AMBER y Morse).

En la primera parte del trabajo se ha estudiado la indeterminación estática y cinemática del modelo mediante una adaptación de la descomposición en valores singulares de Pellegrino y Calladine para estructuras articuladas, con el objetivo descartar posibles modos de mecanismo internos. Este estudio permite establecer las ecuaciones cinemáticas y estáticas de cada elemento constituyente del modelo en el rango de la linealidad, así como plantear las características del ensamblaje de las matrices implicadas $\mathbf{H}$ y $\mathbf{C}$ en el modelo estructural completo. Este primer análisis ha puesto de manifiesto la verificación de la relación de contragradiencia para el modelo MSM y permite establecer la determinación cinemática en función de las condiciones de contorno presentes, así como la alta indeterminación estática interna del sistema.

En una segunda etapa se aborda el análisis geométricamente lineal del modelo mediante una formulación en rigidez completamente general e independiente de la situación de carga deducida a partir de la ecuación de trabajos virtuales. Se proporcionan expresiones cerradas para las matrices de rigidez de cada elemento y se comprueba la verificación del ensamblaje booleano en el modelo. Adicionalmente, se presta especial atención a la curvatura inicial del nanotubo, incluyendo un sistema de esfuerzos iniciales (preeenergía o pretensado) que tiende a estabilizar la sección transversal y acortar el nanotubo. Como parámetros de contraste, se estiman las características mecánicas del nanotubo como conjunto bajo los esquemas de carga habituales en piezas alargadas (tracción, compresión, flexión y torsión), lo cual ha permitido validar la formulación comparando resultados con otros autores, como Natsuki y Endo; todo ello considerando dos potenciales interatómicos distintos, para averiguar la influencia de la no linealidad mecánica en la respuesta final. No obstante, se critica la validez de tales características mecánicas debido a su dependencia de ciertos parámetros geométricos como el espesor de pared equivalente, de determinación controvertida. Adicionalmente, se describen las ventajas del modelo de la tesis en relación a la dudosa aplicación de los modelos continuos clásicos y respecto a los computacionalmente costosos métodos de Dinámica Molecular.

Posteriormente, se lleva a cabo el análisis del comportamiento de SWNTs frente a la inestabili- 
dad desde un punto de vista geométricamente no lineal. El desarrollo de expresiones simbólicas para la matriz de rigidez tangente y la demostración del ensamblaje booleano en el caso no lineal ponen de manifiesto la potencia de la formulación en la obtención de deformaciones críticas con un esfuerzo computacional razonable. Desde el punto de vista numérico, se han implementado algoritmos incrementales-iterativos para cada uno de los potenciales interatómicos considerados, de forma que permitieron verificar que la no linealidad geométrica se desencadena previamente a la constitutiva, de acuerdo con Falvo et al. e Iijima et al. Como resultados se proporcionan las trayectorias de equilibrio no lineales, las deformaciones críticas bajo los esquemas de carga habituales en piezas alargadas y las geometrías deformadas al final del proceso de carga.

Finalmente, se estudia la adecuación de la geometría inicial a la proporcionada por el mapeo conforme sobre una superficie cilíndrica ideal. En concreto, se determinó el diámetro inicial del cilindro mediante la minimización de la energía total del sistema para las tres quiralidades, supuestos nulos los alargamientos de enlace respecto a la longitud de referencia sobre la lámina plana de grafeno. Los diámetros y esfuerzos obtenidos se contrastaron con los resultados del análisis lineal para el modelo MSM, hallando una íntima coincidencia entre los valores de salida de ambos procedimientos. La consideración de esta nueva geometría inicial representa una vía alternativa en la introducción de la curvatura del nanotubo en su comportamiento mecánico.

\subsection{Conclusiones}

\subsubsection{En relación con la formulación del modelo}

1. El modelo stick-spiral admite una formulación general en rigidez para cualquier configuración de cargas externas y condiciones de contorno. El ensamblaje booleano mostrado tanto en el análisis geométricamente lineal como en el no lineal permite la resolución sistemática de estructuras mediante dicho modelo, incluso fuera del marco de la nanotecnología.

2. La introducción de la modificación de longitudes y ángulos de los enlaces respecto de la lámina de grafeno, debido a la curvatura del cilindro, como un sistema de tensiones iniciales es directa en el modelo propuesto, sin más que sumar un sistema de deformaciones iniciales en la ecuación constitutiva (4.5). Esta propiedad representa una importante ventaja de la formulación desarrollada frente a otras técnicas como las MD. Aunque teóricamente existen infinitos sistemas de esfuerzos autoequilibrados en la geometría inicial de cada nanotubo, la estimación realizada en la tesis está asociada con el mapeo conforme. Esto es, asumiendo los átomos de carbono localizados en la superficie cilíndrica y los enlaces covalentes en las secantes entre dos átomos vecinos.

3. La estimación de la geometría inicial mediante un procedimiento de minimización energética representa una vía alternativa en la estimación del sistema de esfuerzos iniciales que pone en entredicho la exactitud de la geometría inicial determinada mediante el mapeo conforme. Teniendo en cuenta que los axiles resultantes al final de dicho procedimiento son despreciables y que los momentos angulares no influyen significativamente en la respuesta mecánica final, estimar adecuadamente la geometría inicial del nanotubo puede traducirse en obviar la introducción de esfuerzos iniciales.

4. Dentro del análisis geométricamente lineal, los códigos propios desarrollados aportan esfuerzos y reacciones, así como las geometrías deformadas finales. Mediante la interpretación cualitativa de éstas deformadas se ha establecido la necesidad de ser extremadamente cautos en la definición de la geometría de SWNTs Chiral, sobre todo en las zonas cercanas a los extremos. En efecto, cerca de los bordes aparecieron deformaciones locales no deseadas, ya que en estas 
zonas algunos de los elementos barra son más cortos y no representan enlaces $C-C$ reales. Por tanto, la aplicación de las cargas externas puede desvirtuar los resultados. En cualquier caso, este problema se puede eliminar introduciendo desplazamientos impuestos en el extremo libre o implementando la geometría de una semiesfera de fullereno coronando el nanotubo.

5. Dentro del análisis geométricamente no lineal, los códigos desarrollados proporcionan trayectorias de equilibrio no lineales, así como geometrías deformadas, esfuerzos internos y reacciones de apoyo al final del último incremento. La respuesta final depende enormemente de las funciones de desplazamientos impuestos, de los valores adoptados para los datos de entrada del proceso incremental-iterativo, del tipo de condiciones de contorno y de las imperfecciones iniciales.

\subsubsection{En relación con los parámetros mecánicos equivalentes}

1. El potencial de Morse proporciona resultados más rígidos a tracción que el AMBER con diferencias en torno al $15 \%$. Idéntico efecto rigidizador producido por la función de Morse (15\% a compresión, hasta un $13 \%$ a flexión y cerca de un $8 \%$ a torsión) está presente en todos los casos de carga estudiados. Sin embargo, no aparecen diferencias importantes para deformaciones por debajo del $6 \%$ a tracción y del $4 \%$ a compresión.

2. Los SWNTs ZigZag son más rígidos que los Armchair a tracción (hasta un $8 \%$ ), pero las diferencias entre ambos a compresión, flexión y torsión no es determinante. No obstante, no se puede afirmar que la respuesta mecánica sea independiente de la quiralidad a flexión y torsión, ya que los valores de $E$ y $G$ obtenidos para los nanotubos Chiral son bastante diferentes a los obtenidos para los nanotubos aquirales bajo dichos estados de carga.

3. El módulo de Young $E$ es ligeramente creciente con el diámetro del nanotubo a tracción y compresión, aunque se puede considerar prácticamente constante en la respuesta longitudinal. Además, $E$ es creciente bajo cargas de flexión y el módulo de deformación transversal $G$ es creciente bajo cargas de torsión con el diámetro del CNT. En ambos casos los resultados de los nanotubos $\mathrm{CH}$ con potencial Morse se ven desvirtuados por el mal condicionamiento numérico experimentado. Esta dificultad puede encontrar su causa en la falta de axisimetría de los nanotubos $\mathrm{CH}$. Al someter estos SWNTs a las acciones externas, algunos enlaces aparecen fuertemente traccionados y otros fuertemente comprimidos. En consecuencia, la extremada diferencia entre las rigideces de enlace a tracción y compresión que proporciona el potencial Morse induce ciertas singularidades numéricas.

4. A pesar de la naturaleza no lineal del potencial Morse, las simulaciones de SWNTs que lo emplean producen una respuesta lineal bajo cualquier caso de carga. Esto es debido a que las rigideces tangentes no cambian de manera significativa en el rango de deformaciones estudiado. No obstante, cabe señalar que los niveles de deformación más elevados quedan fuera del rango de validez del análisis lineal.

5. El pretensado introduce fuerzas resultantes radiales que tienden a mantener la geometría circular de la sección transversal del nanotubo. Así, el efecto Poisson (denominado así por analogía con el efecto del mismo nombre asociado al medio continuo) convierte las fuerzas radiales en acortamiento longitudinal bajo cualquier estado de cargas externas. No obstante, teniendo en cuenta la necesaria modificación del origen de deformaciones longitudinales (comentario 4.3.2), no se produce prácticamente ninguna diferencia en la rigidez a tracción y compresión de SWNTs debido a los esfuerzos iniciales. En general, podemos considerar los casos de carga estudiados como independientes del pretensado. 
6. Aunque se adoptaron los módulos de Young y de deformación transversal como parámetros mecánicos para la comparación de resultados, esta elección puede resultar controvertida debido a su elevada dependencia del espesor de pared equivalente (supuesto habitualmente como 0.34 $\mathrm{nm})$. En nuestro caso, nuestro único objetivo ha sido validar nuestro modelo a partir de los valores de estos parámetros presentes en la literatura mediante formulaciones parciales del modelo atómico stick-spiral, pero no cuantificar los parámetros mecánicos asociados con el continuo.

7. De hecho, es dudoso tratar los SWNTs como un medio continuo, como se ha demostrado a través de la gran dispersión observada en el coeficiente de Poisson obtenido mediante las relaciones constitutivas clásicas de la elasticidad tridimensional.

\subsubsection{En relación con la obtención de deformaciones críticas}

1. En relación a los valores de las deformaciones críticas a compresión, el empleo del potencial AMBER o Morse en el análisis es prácticamente indiferente. Asimismo, a pesar de que los valores obtenidos a flexión y torsión difieren en cierta medida, no se observan diferencias significativas. Por tanto, podemos concluir que la no linealidad constitutiva introducida por el potencial de Morse no influye apreciablemente en la respuesta a la inestabilidad. En consecuencia, la no linealidad geométrica de SWNTs se desarrolla previamente a la no linealidad constitutiva, de acuerdo con Falvo et al., Iijima et al. y Srivastava et al.

2. Teniendo en cuenta que las imperfecciones de la estructura atómica de los SWNTs son extremadamente condicionantes en la respuesta frente a la inestabilidad y en la determinación de deformaciones críticas, el modelo stick-spiral constituye una importante herramienta en el análisis geométricamente no lineal de nanotubos. En particular, los procedimientos incrementalesiterativos diseñados permiten la introducción de cualquier sistema de imperfecciones iniciales, en contraste con la incapacidad de los modelos continuos para plasmar tales imperfecciones. Éstas se introducen entonces mediante métodos artificiosos, lo que se traduce en una reducción importante de la precisión y resultados carentes de sentido físico.

3. Como se ha mostrado, nuestros resultados son razonables en comparación con algunas referencias que emplean modelos atómicos. Por otra parte, a pesar de la dudosa aplicabilidad de los modelos continuos a sistemas atómicos, algunos autores como Yakobson et al. los han empleado adoptando valores apropiados para algunos parámetros mecánicos con el objetivo de validar sus cálculos MD. En esta línea, hemos utilizado algunas de sus ecuaciones del continuo para comparar cualitativamente con nuestros resultados obtenidos, llegando a una coincidencia razonable a compresión (error relativo máximo alrededor del 20\%). No obstante, sólo se consigue una correspondencia cualitativa, en términos de orden de magnitud, a flexión y torsión.

4. Respecto al comportamiento postcrítico final, el reducido tamaño de los SWNTs estudiados hace que algunas deformadas queden desvirtuadas, alejándose de los patrones de deformación clásicos frente a la inestabilidad. En concreto, los efectos de borde y la mayor influencia relativa de la falta de axisimetría se verían previsiblemente reducidos en SWNTs de mayores dimensiones. 


\subsection{Aportaciones originales}

Describimos a continuación las aportaciones originales realizadas en la tesis:

1. La adaptación de la descomposición SVD al modelo stick-spiral, aunque está basada en la idea original de Pellegrino [84], [85], requiere el desarrollo de la cinemática del elemento muelle que conecta dos barras consecutivas. Esto implica la definición de la deformación angular $\epsilon$ y la obtención explícita de las funciones $\boldsymbol{\Gamma}$, así como la definición del ensamblaje de las matrices cinemáticas y de equilibrio que permite la inclusión del resorte en la formulación. La mayor dificultad derivada de incorporar resortes angulares es la inconsistencia de grados de libertad; es decir, el modelo MSM sólo admite desplazamientos y fuerzas nodales lineales (no angulares) en los nudos, pero la deformación angular intrínseca al elemento muelle debe expresarse en función de dichos desplazamientos.

2. La idea del desarrollo de una formulación general en rigidez del modelo que permite desacoplar las contribuciones de cada elemento estructural y verificar la adecuación del ensamblaje booleano, a partir de un procedimiento energético que implica la aplicación sistemática de la ETV a uno o varios elementos estructurales. En efecto, aunque el proceso descrito es ampliamente conocido en mecánica del continuo e ingeniería estructural, la mayor parte de referencias consultadas que tratan sobre el modelo stick-spiral [14], [15], [77], [78] desarrollan formulaciones parciales mediante complejos razonamientos trigonométricos que aprovechan ciertas condiciones de simetría del nanotubo por simplicidad. De hecho, cada configuración de cargas externas requiere una nueva formulación de las ecuaciones necesarias. Sólo Meo y Rossi [74] tratan SWNTs de forma general mediante el presente modelo, aunque implementado en un código comercial de elementos finitos (con la falta de precisión inherente al método) y sin considerar la no linealidad constitutiva en la distorsión angular. En definitiva, la falta de una formulación general del modelo era patente.

3. La introducción directa del pretensado como un sistema de deformaciones iniciales en la formulación es nueva en las técnicas de análisis a nivel molecular. Los autores que emplean los modelos MSM simplemente desprecian éstos esfuerzos iniciales, aunque en las técnicas MD son considerados habitualmente a partir de la diferencia de energía de deformación entre el SWNT y la lámina plana de grafeno. Introducir el pretensado en la formulación lineal de la tesis se reduce a sumar un término de esfuerzos iniciales en las ecuaciones consitutivas (4.6), pero tiene el inconveniente de requerir una estimación físicamente razonable del sistema de esfuerzos iniciales.

4. El desarrollo de la cinemática no lineal para los elementos barra y muelle, así como la obtención de las expresiones simbólicas de la matriz de rigidez tangente de cada elemento a partir de las segundas variaciones del trabajo de las fuerzas internas es una de las novedades importantes en el modelo stick-spiral.

5. La adaptación de los conocidos algoritmos incrementales-iterativos al modelo stick-spiral para analizar el comportamiento no lineal geométrico de SWNTs es novedoso. También se idearon algunas mejoras en el rendimiento numérico de dichos algoritmos, sobre todo en la obtención de la velocidad incremental y la resolución del sistema incremental de rigidez, que representan el mayor consumo en tiempo de computación. El desarrollo de estos algoritmos y el trazado de las trayectorias de equilibrio nos permite la aplicación del modelo a la obtención de deformaciones críticas.

6. Teniendo en cuenta el acortamiento que produce el pretensado, las modificaciones en la rigidez longitudinal pasan por una determinación cuidadosa de la geometría inicial. En esta dirección, 
el desarrollo del proceso de minimización energética que aproxima el diámetro inicial con mayor precisión que el mapeo conforme constituye una idea novedosa. De hecho, puede considerarse como una alternativa respecto a la introducción del sistema de esfuerzos iniciales, siempre que dispongamos del mapeo adecuado.

\subsection{Líneas de investigación propuestas}

\subsubsection{Introducción del pretensado en el análisis geométricamente no lineal}

Dada la pequeña influencia de la preenergía observada en en análisis geométricamente lineal (capítulo 4), y teniendo en cuenta que sólo habrá diferencias notables en los SWNTs de diámetro más reducido (poco habituales experimentalmente), en el análisis geométricamente no lineal (capítulo 5) se ha obviado la introducción de esfuerzos iniciales. Sin embargo, una posible extensión de la formulación desarrollada consiste en incluir la predeformación en los algoritmos numéricos planteados.

\section{Implementación numérica}

Una vez fijado el diámetro del nanotubo, cada SWNT aislado estará provisto de un valor fijo de la preenergía y por tanto de los esfuerzos y deformaciones asociados. En consecuencia, parece lógico introducir un incremento cero regulado por la estrategia de control de carga estándar [29], previamente al diagrama de flujo general plasmado en la figura 5.2 orientado a incluir esta predeformación.

Puesto que experimentalmente, ya sea durante el proceso de fabricación o en el interior de un polímero reforzado con nanotubos, la preenergía actúa previamente a la actuación de cualquier restricción externa, las ideas expuestas en esta línea de investigación sólo tendrían sentido si se trataran posteriormente a la aplicación de condiciones de contorno. Esto constituye una vía para investigaciones futuras, no obstante desarrollamos algunos conceptos aquí como paso previo.

Dado que el pretensado actúa previamente a cualquier carga externa, la hipótesis "inicial"para $\mathbf{u}_{n}^{0}, \lambda_{n}^{0}, \mathbf{P}_{n}^{0}$ en la figura 5.2 adopta valores no nulos, que deberán obtenerse mediante el incremento cero con control de carga. Este tipo de control es el empleado por el denominado método de Newton ordinario y se puede considerar como un caso particular de los métodos numéricos incrementalesiterativos para el que la ecuación de restricción implica un sistema de cargas externas constante. En este caso, la ec. (5.66) se convierte en:

$$
c\left(\Delta \mathbf{u}_{n}, \Delta \lambda_{n}\right)=\Delta \lambda_{n}-l_{0}=0
$$

luego la ecuación (5.67b) se reduce a:

$$
c_{n}^{k+1}=c_{n}^{k}+\Delta \lambda_{n}^{k}
$$

donde $c_{n}^{k} \equiv 0$ porque la ecuación de restricción se satisface idénticamente y el sistema (5.68) se simplifica según:

$$
\left[\begin{array}{cc}
\mathbf{K}\left(\mathbf{u}_{n}^{k}\right) & -\mathbf{q}_{n}^{k} \\
\mathbf{0} & 1
\end{array}\right]\left\{\begin{array}{l}
\mathbf{d}_{n}^{k} \\
\eta_{n}^{k}
\end{array}\right\}=-\left\{\begin{array}{c}
\mathbf{r}_{n}^{k} \\
0
\end{array}\right\}
$$

luego $\eta_{n}^{k}=0$. Es decir, el vector de carga incremental desaparece completamente del proceso iterativo y la iteración del Newton queda:

$$
\mathbf{d}_{n}^{k}=-\mathbf{K}\left(\mathbf{u}_{n}^{k}\right)^{-1} \mathbf{r}_{n}^{k}
$$


Con una estructura muy similar al diagrama general de la figura 5.2, representamos en la figura 7.1 el diagrama de flujo del incremento cero, cuyas fases predictiva y correctiva se detallan en la figura 7.2 .

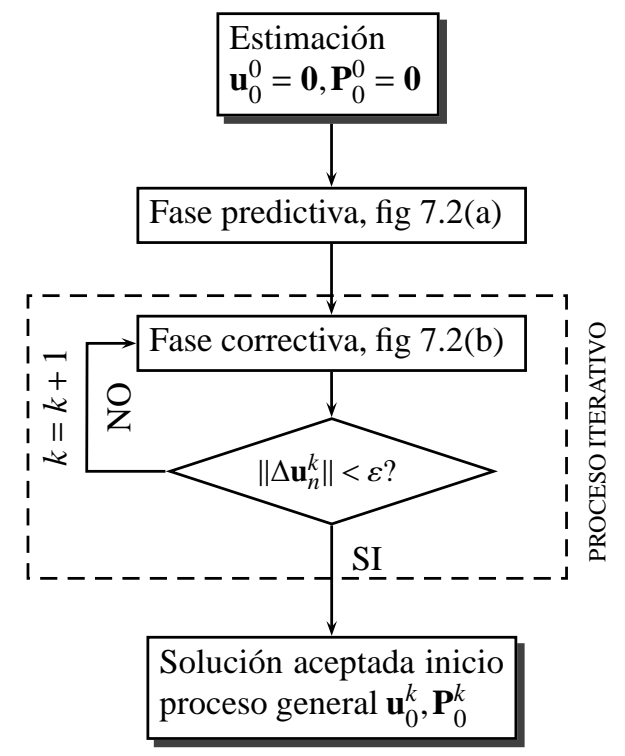

Figura 7.1: Diagrama de flujo incremento cero

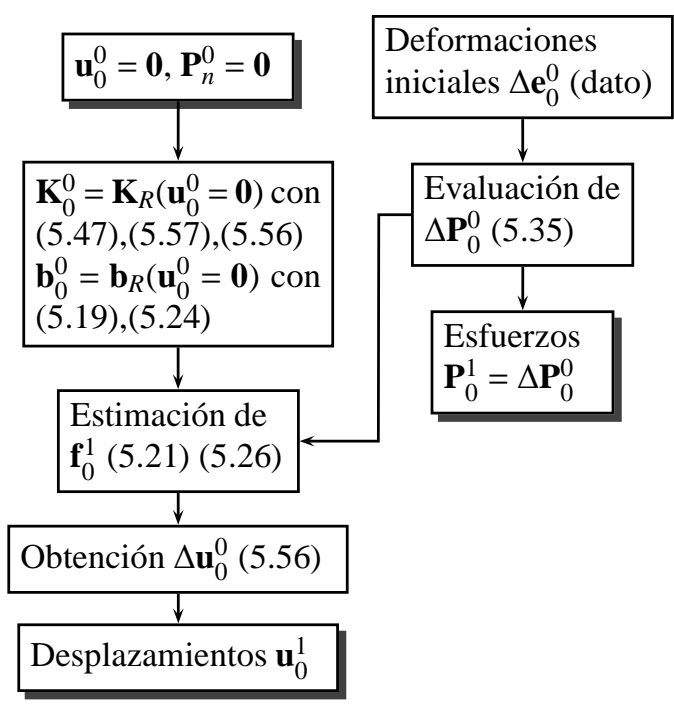

(a) Fase predictiva (incremental)

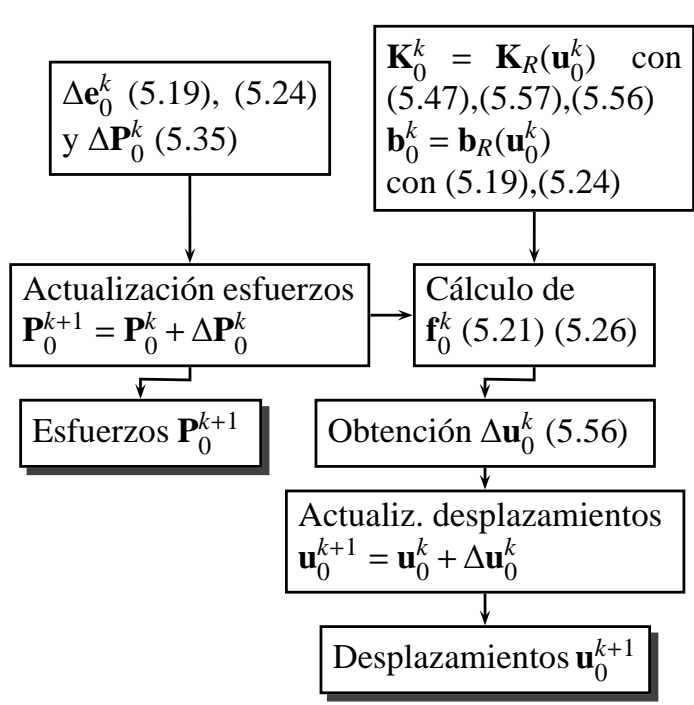

(b) Fase correctiva (iterativa)

Figura 7.2: Incremento $(n=0)$, estrategia de control de fuerza 
En cualquier caso, conviene añadir los siguientes comentarios aclaratorios:

1. Tal como hicimos con el diagrama de flujo general de las figuras 5.2 y 5.3 , hemos desarrollado dos códigos diferentes para el incremento de pretensado, uno con potencial AMBER 4.12b) el otro con potencial Morse (4.14). Las rigideces tangentes dentro del incremento cero son coherentes con las incluidas en el procedimiento general.

2. Aunque los desplazamientos u se miden desde la geometría inicial del nanotubo, tanto los esfuerzos iniciales $\mathbf{P}_{0}$ como sus deformaciones asociadas $\boldsymbol{\epsilon}_{0}$ se miden respecto a las longitudes de enlace y ángulos correspondientes a la posición de equilibrio (no tensionada) del potencial. Dicha geometría de equilibrio es coincidente con la de la lámina de grafeno en ausencia de cargas externas.

3. El sistema de rigidez incremental (5.56) adopta la siguiente forma:

$$
\begin{gathered}
\Delta \mathbf{f}_{0}^{k}=\overline{\mathbf{f}}_{0}^{k}-\mathbf{f}_{0}^{k}=\mathbf{K}_{0}^{k} \Delta \mathbf{u}_{0}^{k} \\
-\mathbf{f}_{0}^{k}=\mathbf{K}_{0}^{k} \Delta \mathbf{u}_{0}^{k}
\end{gathered}
$$

En efecto, las cargas externas impuestas en los nudos libres $\overline{\mathbf{f}}_{0}^{k}$ se anulan en este incremento cero por hipótesis. Incluso las fuerzas $\mathbf{f}_{0}^{k}$ tienden a cero en la convergencia de la solución, porque los esfuerzos internos correspondientes a la preenergía deben estar autoequilibrados.

\subsubsection{Deducción del mapeo generador de la geometría inicial}

Como se ha visto en el capítulo 6 es posible obtener geometrías sensiblemente cilíndricas relajadas respecto a la del mapeo conforme, donde los enlaces no sufran acortamiento alguno. Además, se ha mostrado la coincidencia de los resultados obtenidos (tanto en la estimación de diámetros como en esfuerzos) con los correspondientes al modelo MSM propuesto.

Aunque se ha aceptado por hipótesis que la orientación relativa de los enlaces respecto a las direcciones longitudinal y transversal al nanotubo es invariable en el proceso de mapeado de la lámina de grafeno sobre el cilindro, la deducción simbólica de dicho mapeo ha quedado pospuesta a futuras investigaciones. En esta línea, cabría investigar también las posibles modificaciones en términos de esfuerzos y deformaciones iniciales causadas por diferentes mapeos iniciales (incluso con el mismo diámetro inicial).

La definición simbólica de un mapeo inicial que permita estimar los esfuerzos iniciales con suficiente precisión permitiría (por ejemplo) evitar el trabajo numérico de implementar un incremento $(n=0)$ en el marco de los procedimientos incrementales-iterativos, reduciendo el tiempo de computación a costa de un mayor esfuerzo teórico.

\subsubsection{Extensión de la formulación a la dinámica}

En general, el equilibrio dinámico de cualquier sistema estructural de $N$ grados de libertad se puede escribir:

$$
\mathbf{f}_{I}+\mathbf{f}_{D}+\mathbf{f}_{S}=\mathbf{p}(t)
$$

donde:

$\mathbf{f}_{I} \quad$ Fuerzas de inercia

$\mathbf{f}_{D} \quad$ Fuerzas de amortiguamiento

$\mathbf{f}_{s} \quad$ Fuerzas elásticas

$\mathbf{p}(t) \quad$ Excitación externa 
que en términos del campo de desplazamientos $\mathbf{v}$ será

$$
\mathbf{m} \ddot{\mathbf{v}}+\mathbf{c} \dot{\mathbf{v}}+\mathbf{k v}=\mathbf{p}(t)
$$

donde:

m Matriz de coeficientes de influencia másicos

c Matriz de coeficientes de influencia de amortiguamiento

k Matriz de rigidez

( ) $=\frac{\partial}{\partial t} \quad$ Derivación respecto al tiempo

Por tanto, el equilibrio dinámico del modelo estructural stick-spiral también será susceptible de ser expresado en la forma (7.7), definiendo adecuadamente los parámetros implicados en su análisis. Así, la extensión dinámica de la formulación desarrollada en la tesis constituye otra importante línea de investigación.

La obtención de la matriz de rigidez $\mathbf{k}$ es inmediata a partir del ensamblaje de las matrices de rigidez de los elementos estructurales individuales, tal como se ha demostrado en la tesis. Respecto a la obtención de los coeficientes másicos, resulta físicamente razonable localizar las masas de los átomos de $\mathrm{C}$ en los nudos, de forma que no posean inercia rotacional (masas puntuales). De esta forma, la matriz $\mathbf{m}$ es diagonal [19, §11-2] y adopta una forma:

$$
\mathbf{m}=m_{0} \mathbf{I}
$$

donde $m_{0} \simeq 2 \cdot 10^{-23} \mathrm{~g}$ es la masa de un átomo de $\mathrm{C}$, calculada como el cociente del peso molecular del carbono y el número de Avogadro.

Para la determinación de los coeficientes de amortiguamiento, se requerirán experimentos específicos que permitan su calibración a escala interatómica. En su defecto, suponiendo que todos los enlaces covalentes $\mathrm{C}$ - $\mathrm{C}$ tienen el mismo amortiguamiento longitudinal $c^{\lambda}$ y que todas las interacciones de tres cuerpos tienen el mismo amortiguamiento rotacional $c^{\Gamma}$, dispondríamos de dos constantes a determinar mediante un contraste paramétrico, con la consiguiente comparación de valores de frecuencias y aceleraciones máximas obtenidas, en relación a experimentos sobre SWNTs ensayados frente a vibraciones.

La determinación de las excitaciones externas será probablemente una cuestión controvertida. Si las acciones externas están aplicadas directamente sobre los nudos, el vector $\mathbf{p}(t)$ se puede escribir directamente, como cabe esperar en el modelo MSM. No obstante, la traducción de una excitación térmica (empleada por [17]) o electrostática a un conjunto de excitaciones mecánicas sobre los nudos del sistema presenta un fuerte reto en esta línea de investigación.

Una vez determinado los coeficientes implicados en la forma (7.7) del equilibrio dinámico del sistema, su integración en el tiempo y la determinación de frecuencias propias de vibración así como de aceleraciones y desplazamientos máximos representará con toda seguridad un nuevo punto de vista en el comportamiento mecánico de estas singulares moléculas.

\subsubsection{Análisis estructural de MWNTs y nanosistemas}

Algunos autores [59], [120] han empleado modelos MSM para analizar el comportamiento estructural de sistemas formados por SWNTs, tales como MWNTs o el denominado buckypaper, que consiste en un sistema de fajos de MWNTs enlazados entre sí mediante interacciones de VDW formando una especie de papel poroso. En particular, cada capa de un MWNT es analizada como un sistema de barras rígidas con parámetros de rigidez equivalente derivados del potencial interatómico, mientras las conexiones entre capas asociadas a la interacción de VDW se reproducen mediante elementos biarticulados que conectan cada átomo de una capa con los inmediatos vecinos de la contigua. 
Por tanto, dada la similitud entre el modelo de barras rígidas descrito y el modelo stick-spiral, parece lógico extender la escala de los sistemas moleculares representados mediante la misma estrategia utilizada por Li y Chow [59]. El mayor inconveniente que se presenta es el coste computacional por el elevado número de grados de libertad implicado, aunque en el caso del stick-spiral es del orden de $3 N$ frente a los $6 N$ del modelo MSM de barras rígidas. Para resolverlo, siempre es posible recurrir a la condensación estática que permitiría por ejemplo, determinar la rigidez longitudinal de los MWNTs sin necesidad de emplear el controvertido espesor de pared equivalente.

En la misma línea, analizar estructuralmente sistemas como el buckypaper mediante la sucesiva condensación estática de moléculas y sistemas presenta una alternativa al método multiescala empleado en [120], que se limita a obtener la rigidez equivalente de cada sistema molecular mediante ensayos particulares y utilizar los resultados obtenidos como punto de partida en el análisis de otro sistema de mayor tamaño cuya unidad elemental es la molécula inicial.

\subsubsection{Introducción de la interacción con fluidos y otros entornos}

Desde el punto de vista estructural, el comportamiento de polímeros reforzados con nanotubos representa un prometedor campo de aplicaciones tecnológicas, sobre todo en aquellos campos donde se requiere un elevado ratio entre resistencia y peso del material (industria aeronáutica, robótica, etc). Una vez determinada la resistencia de la conexión electroquímica nanotubo-matriz, introducir dicha interacción en el modelo MSM para analizar sistemas complejos es uno de los mayores campos potenciales de aplicación de los modelos a escala atómica.

Asimismo, la introducción de la interacción electrostática en el cálculo (probablemente mediante la conversión a cargas estáticas o excitaciones dinámicas equivalentes), permitiría aplicar el modelo MSM al análisis del acoplamiento electro-mecánico de nanotubos, tratado en algunas referencias por otras vías [90].

Una línea algo más alejada del análisis puramente estructural consiste en introducir la interacción del modelo con fluidos. Las aplicaciones de tal análisis varían desde el diseño de músculos artificiales embebidos en sangre [67] hasta la investigación en el funcionamiento de microcapilares o la distribución de medicamentos intravenosos, tales como la quimioterapia [57]. 


\section{Apéndice A}

\section{Notación y Acrónimos}

\section{A.1. Notación}

Para facilitar la lectura de la Tesis y la consulta de algunas variables presentes en una buena parte de la formulación, incluimos a continuación un listado con las entidades matemáticas más importantes definidas en el texto.

\begin{tabular}{ll}
\hline $\mathbf{a}_{1}, \mathbf{a}_{2}$ & Vectores base de la malla hexagonal \\
$\mathbf{C}_{h}$ & Vector Quiral (o Chiral) \\
$n$ & $\mathrm{n}^{0}$ de hexágonos en la dirección ZigZag \\
$m$ & $\mathrm{n}^{0}$ de hexágonos en la dirección del vector Quiral \\
$\mathbf{T}$ & Vector traslación \\
$a_{0}$ & Longitud de equilibrio del enlace covalente, normalmente $0^{\prime} 142 \mathrm{~nm}$ \\
$\theta$ & Ángulo Quiral \\
$D_{t}, d$ & Diámetro del SWNT \\
$t$ & Separación entre láminas de grafeno, normalmente $0,34 \mathrm{~nm}$ \\
\hline
\end{tabular}

Tabla A.1: Variables que definen la geometría de los SWNTs

\begin{tabular}{ll}
\hline$a, b, c, \ldots$ & Emplearemos superíndices alfabéticos para los elementos barra \\
$\lambda^{a}$ & Vector unitario de barra \\
$l^{a}$ & Longitud inicial de la barra \\
$\mathbf{u}^{a}$ & Desplazamientos nodales del elemento barra \\
$\epsilon^{a}=d l^{a}$ & Deformación o alargamiento de barra (análisis lineal) \\
$n^{a}$ & Axil de barra (positivo tracción) \\
$\mathbf{C}_{l}^{a}$ & Matriz cinemática de barra (análisis lineal) \\
$\mathbf{H}_{l}^{a}$ & Matriz de equilibrio de barra (análisis lineal) \\
$\mathbf{f}_{i}^{a}, \mathbf{f}_{j}^{a}$ & Fracción de las fuerzas externas que colaboran al alargamiento de barra \\
\hline
\end{tabular}

Tabla A.2: Variables asociadas a los elementos barra del modelo 


\begin{tabular}{ll}
\hline $1,2,3, \ldots$ & Emplearemos superíndices numéricos para los elementos muelle \\
$A, \alpha$ & Ángulos inicial y final respectivamente \\
$\boldsymbol{\Gamma}_{j}^{1}, \boldsymbol{\Gamma}_{i}^{1}, \boldsymbol{\Gamma}_{k}^{1}$ & Funciones vectoriales características del muelle \\
$\mathbf{u}^{1}$ & Desplazamientos nodales del elemento muelle \\
$\epsilon^{1}=d \gamma^{1}$ & Distorsión angular de muelle (análisis lineal) \\
$m^{1}$ & Momento de muelle (positivo si tiende a cerrarlo) \\
$\mathbf{C}_{l}^{1}$ & Matriz cinemática de muelle (análisis lineal) \\
$\mathbf{H}_{l}^{1}$ & Matriz de equilibrio de muelle (análisis lineal) \\
$\mathbf{f}_{j}^{1}, \mathbf{f}_{i}^{1}, \mathbf{f}_{k}^{1}$ & Fracción de las fuerzas externas que colaboran a la distorsión de muelle \\
\hline & Tabla A.3: Variables asociadas a los elementos muelle del modelo
\end{tabular}

\begin{tabular}{ll}
\hline $\mathbf{x}_{i}$ & Vector de posición del nudo i \\
$\mathbf{u}_{i}$ & Desplazamientos nodales del nudo i \\
$\mathbf{u}$ & Desplazamientos nodales del sistema \\
$\mathbf{u}_{R}$ & Desplazamientos nodales de g.d.l. no coaccionados \\
$\mathbf{u}_{A}$ & Desplazamientos nodales de g.d.l. coaccionados \\
$\mathbf{C}_{l}$ & Matriz cinemática del sistema \\
$\mathbf{C}_{R}$ & Matriz cinemática del sistema asociada a g.d.l. no coaccionados \\
$\mathbf{C}_{A}$ & Matriz cinemática del sistema asociada a g.d.l. coaccionados \\
$\mathbf{C}^{\lambda}$ & Matriz cinemática del sistema correspondiente a las deformaciones longitudinales de barra \\
$\mathbf{C}^{\Gamma}$ & Matriz cinemática del sistema correspondiente a las distorsiones angulares de muelle \\
$\boldsymbol{\epsilon}$ & Vector deformación del sistema \\
$\boldsymbol{\epsilon}^{\lambda}$ & Vector de alargamientos de barra \\
$\boldsymbol{\epsilon}^{\Gamma}$ & Vector de distorsiones de muelle \\
\hline
\end{tabular}

Tabla A.4: Variables cinemáticas (análisis lineal) asociadas al sistema estructural completo

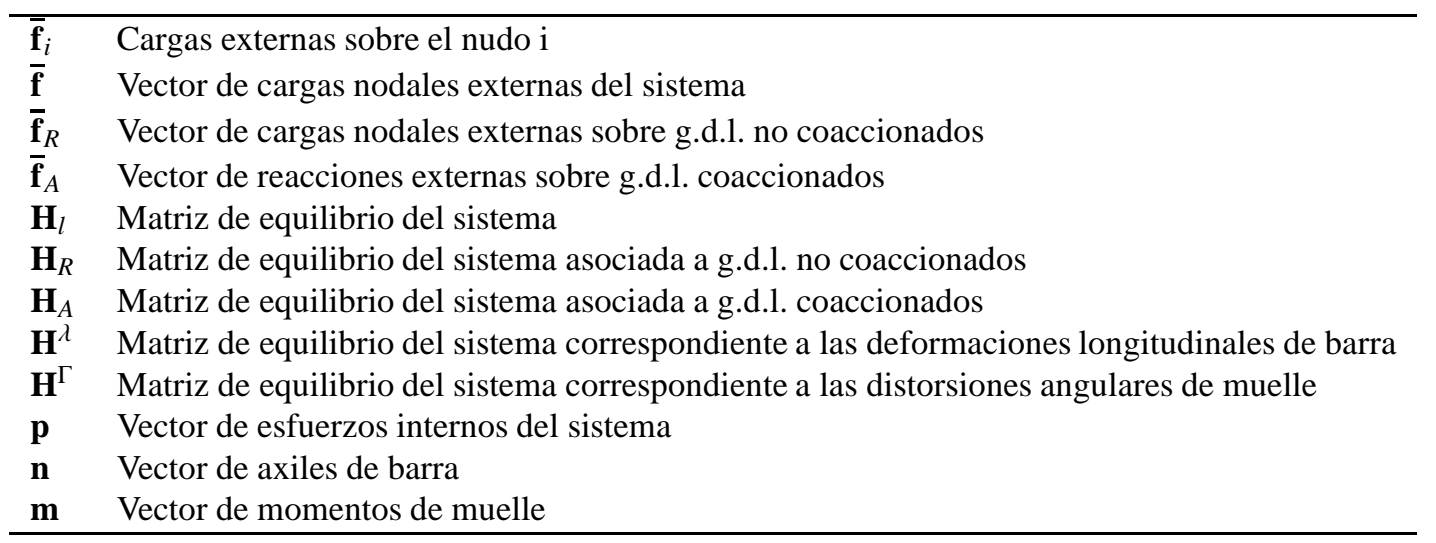

Tabla A.5: Variables estáticas (análisis lineal) asociadas al sistema estructural completo 


\begin{tabular}{ll}
\hline$F^{a}$ & Flexibilidad longitudinal de barra \\
$F^{1}$ & Flexibilidad angular de muelle \\
$\mathbf{F}$ & Matriz de flexibilidad del sistema (supuesta diagonal) \\
$K^{a}$ & Rigidez longitudinal de barra \\
$K^{1}$ & Rigidez angular de muelle \\
$\mathbf{K}=\mathbf{F}^{-1}$ & Matriz constitutiva (diagonal) del sistema, \\
& incluye las rigideces individuales de cada elemento \\
$\mathbf{K}^{\lambda}$ & Bloque (diagonal) de $\mathbf{K}$ con las rigideces de barra \\
$\mathbf{K}^{\Gamma}$ & Bloque (diagonal) de K con las rigideces de muelle \\
$\boldsymbol{\epsilon}_{0}^{a}$ & Predeformación de barra (origen en el SWNT) \\
$\boldsymbol{\epsilon}_{0}^{1}$ & Predeformación de muelle (origen en el SWNT) \\
$\boldsymbol{\epsilon}_{0}$ & Vector de predeformaciones (origen en el SWNT) \\
$n_{0}^{a}$ & Axil inicial de pretensado \\
$m_{0}^{1}$ & Momento inicial de pretensado \\
$\mathbf{p}_{0}$ & Vector de esfuerzos de pretensado \\
$\mathbf{n}_{0}$ & Vector de axiles de pretensado \\
$\mathbf{m}_{0}$ & Vector de momentos de pretensado \\
$K^{\lambda}$ & Rigidez tangente de barra \\
$K^{\Gamma}$ & Rigidez tangente de muelle \\
$\Delta r$ & Alargamiento de barra entre dos configuraciones cualesquiera \\
$\Delta \theta$ & Distorsión de muelle entre dos configuraciones cualesquiera \\
\hline
\end{tabular}

Tabla A.6: Magnitudes y parámetros incluidas en las ecuaciones constitutivas

\begin{tabular}{ll}
\hline$\widetilde{\mathbf{K}}_{l}^{a}$ & Matriz de rigidez del elemento barra \\
$\widetilde{\mathbf{K}}_{l}^{1}$ & Matriz de rigidez del elemento muelle \\
$\widetilde{\mathbf{K}}_{l}$ & Matriz de rigidez global del sistema \\
$\widetilde{\mathbf{K}}_{R}$ & Matriz de rigidez reducida del sistema \\
$\widetilde{\mathbf{K}}_{A}, \widetilde{\mathbf{K}}_{R A}, \widetilde{\mathbf{K}}_{A R}$ & Resto de bloques de la matriz de rigidez global \\
$\mathbf{f}_{0}^{a}$ & Fracción de las fuerzas de apoyo perfecto debidas al pretensado \\
& y asociadas al alargamiento de barra \\
$\mathbf{f}_{0}^{1}$ & Fracción de las fuerzas de apoyo perfecto debidas al pretensado \\
$\mathbf{f}_{0}$ & y asociadas a la distorsión de muelle \\
$\mathbf{f}_{0 R}$ & Fuerzas de apoyo perfecto debidas al pretensado \\
$\mathbf{f}_{0 A}$ & Fuerzas de apoyo perfecto debidas al pretensado sobre g.d.l. no coaccionados \\
\hline
\end{tabular}

Tabla A.7: Magnitudes incluidas en la formulación en rigidez, análisis lineal geométrico 


\begin{tabular}{ll}
\hline()$^{\prime}$ & Aplicable a las variables en la geometría deformada \\
$e^{a}$ & Deformación no lineal del elemento barra \\
$e^{1}$ & Deformación no lineal del elemento muelle \\
$\mathbf{e}^{\lambda}$ & Vector de deformaciones no lineales de barra \\
$\mathbf{e}^{\Gamma}$ & Vector de deformaciones no lineales de muelle \\
$\mathbf{e}=\left\{\mathbf{e}^{\lambda}, \mathbf{e}^{\Gamma}\right\}^{T}$ & Vector de deformaciones no lineales sistema completo \\
$\mathbf{Z}^{a}$ & Matriz auxiliar constante asociada a la cinemática de barra $(6 \times 6)$ \\
$\mathbf{Z}^{1}$ & Matriz auxiliar constante asociada a la cinemática de muelle $(9 \times 9)$ \\
$\mathbf{C}_{n}^{a}\left(\mathbf{u}^{a}\right)$ & Matriz cinemática no lineal del elemento barra \\
$\mathbf{C}_{n}^{1}\left(\mathbf{u}^{1}\right)$ & Matriz cinemática no lineal del elemento muelle \\
$\mathbf{C}^{a}\left(\mathbf{u}^{a}\right)$ & Matriz cinemática total del elemento barra \\
$\mathbf{C}^{1}\left(\mathbf{u}^{1}\right)$ & Matriz cinemática total del elemento muelle \\
$\mathbf{b}^{a}\left(\mathbf{u}^{a}\right)$ & Matriz cinemática tangente del elemento barra \\
$\mathbf{b}^{1}\left(\mathbf{u}^{1}\right)$ & Matriz cinemática tangente del elemento muelle \\
$\mathbf{b}(\mathbf{u})$ & Matriz cinemática tangente del sistema estructural completo \\
\hline
\end{tabular}

Tabla A.8: Variables cinemáticas, análisis no lineal geométrico

\begin{tabular}{ll}
\hline$N^{a}$ & Axil de barra retrotraído a la configuración inicial \\
$M^{1}$ & Momento de muelle retrotraído a la configuración inicial \\
$\mathbf{N}$ & Vector de axiles de barra retrotraídos a la configuración inicial \\
$\mathbf{M}$ & Vector de momentos de muelle retrotraídos a la configuración inicial \\
$\mathbf{P}=\{\mathbf{N}, \mathbf{M}\}^{T}$ & Vector de esfuerzos retrotraídos a la configuración inicial \\
$\mathbf{H}_{n}^{a}\left(\mathbf{u}^{a}\right)$ & Matriz de equilibrio no lineal de barra \\
$\mathbf{H}_{n}^{1}\left(\mathbf{u}^{1}\right)$ & Matriz de equilibrio no lineal de muelle \\
$\mathbf{H}_{n}(\mathbf{u})$ & Matriz de equilibrio no lineal del sistema completo \\
$\mathbf{H}^{a}\left(\mathbf{u}^{a}\right)=\mathbf{b}^{a T}\left(\mathbf{u}^{a}\right)$ & Matriz de equilibrio total de barra \\
$\mathbf{H}^{1}\left(\mathbf{u}^{1}\right)=\mathbf{b}^{1}\left(\mathbf{u}^{1}\right)$ & Matriz de equilibrio total de muelle \\
$\mathbf{H}(\mathbf{u})=\mathbf{b}^{T}(\mathbf{u})$ & Matriz de equilibrio total del sistema completo \\
$\mathbf{f}_{R}$ & Vector de fuerzas externas aplicadas en los nudos libres \\
$\mathbf{f}_{A}$ & Vector de reacciones en los apoyos fijos \\
$\mathbf{f}_{M}$ & Vector de reacciones en los apoyos móviles \\
\hline
\end{tabular}

Tabla A.9: Variables estáticas, análisis no lineal geométrico

$K^{a}\left(e^{a}\right) \quad$ Rigidez constitutiva tangente del elemento barra

$K^{1}\left(e^{1}\right) \quad$ Rigidez constitutiva tangente del elemento muelle

$\mathbf{K}^{\lambda}\left(\mathbf{e}^{\lambda}\right) \quad$ Matriz constitutiva tangente asociada a las deformaciones de barra

$\mathbf{K}^{\Gamma}\left(\mathbf{e}^{\Gamma}\right)$ Matriz constitutiva tangente asociada a las deformaciones de muelle

Tabla A.10: Magnitudes ecs. constitutivas incrementales, análisis no lineal geométrico 


\begin{tabular}{ll}
\hline $\mathbf{K}_{T}^{a}$ & Matriz de rigidez tangente del elemento barra \\
$\mathbf{K}_{T}^{1}$ & Matriz de rigidez tangente del elemento muelle \\
$\mathbf{K}_{M}^{a}\left(\mathbf{u}^{a}\right)$ & Matriz de rigidez material del elemento barra \\
$\mathbf{K}_{M}^{1}\left(\mathbf{u}^{1}\right)$ & Matriz de rigidez material del elemento muelle \\
$\mathbf{K}_{G}^{a}\left(N^{a}\right)$ & Matriz de rigidez geométrica del elemento barra \\
$\mathbf{K}_{G}^{1}\left(M^{1}\right)$ & Matriz de rigidez geométrica del elemento muelle \\
$\Delta \mathbf{u}_{M}=\Delta \overline{\mathbf{u}}$ & Incremento de desplazamientos impuestos en los apoyos móviles \\
$\lambda$ & Parámetro o factor de carga \\
$\mathbf{d}=\Delta \mathbf{u}$ & Incremento del campo de desplazamientos \\
$\eta=\Delta \lambda$ & Incremento del factor de carga \\
$\overline{\mathbf{d}}$ & Desplazamientos incrementales apoyos móviles \\
$\mathbf{q}$ & Vector de carga incremental general \\
$\overline{\mathbf{q}}$ & Vector de carga incremental equivalente a los desplazamientos $\widetilde{\mathbf{d}}$ \\
$\overline{\mathbf{q}}_{\epsilon}$ & Vector de imperfección incremental inicial \\
$\mathbf{r}(\mathbf{u}, \lambda)$ & Residuo del sistema incremental \\
$c(\Delta \mathbf{u}, \Delta \lambda)$ & Coacción determinada por la estrategia de control \\
$\mathbf{v}$ & Vector velocidad incremental \\
$f$ & Factor de escala del vector tangente a la trayectoria de equilibrio \\
$l_{0}$ & Tamaño de incremento \\
$N I$ & Número de incrementos a trazar en la trayectoria \\
()$_{n}^{k}$ & Variable en la iteración $k$ del incremento $n$ \\
\hline
\end{tabular}

Tabla A.11: Variables sistema incremental de rigidez, análisis no lineal geométrico

\begin{tabular}{ll}
\hline$d_{0}$ & Diámetro inicial del nanotubo en ausencia de cargas externas \\
$R=d_{0} / 2$ & Radio inicial del nanotubo en ausencia de cargas externas \\
$n_{0}^{i}$ & Axiles iniciales en la barra $i=a, b, c$ del modelo (axiles del pretensado, ver tabla A.6) \\
$m_{0}^{j}$ & $\begin{array}{l}\text { Momentos iniciales en el resorte angular } j=1,2 \text { del modelo (momentos } \\
\text { del pretensado, ver tabla A.6) }\end{array}$ \\
\hline
\end{tabular}

Tabla A.12: Determinación diámetro inicial por minimización energética, magnitudes comunes

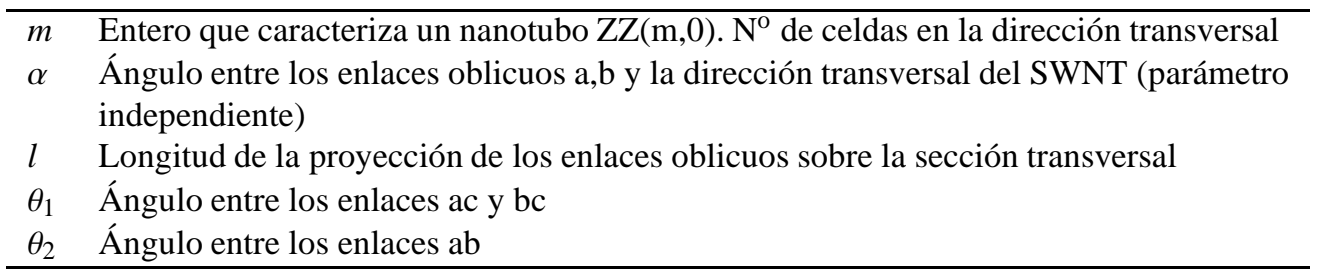

Tabla A.13: Determinación diámetro inicial por minimización energética, SWNTs ZigZag 
$m$ Entero que caracteriza un nanotubo $\mathrm{AC}(\mathrm{m}, 0)$. Coincide con la mitad del número de celdas en la dirección transversal

$\alpha \quad$ Ángulo entre los enlaces oblicuos a,c y la dirección longitudinal al SWNT (parámetro independiente)

$l \quad$ Longitud de la proyección de los enlaces oblicuos sobre la sección transversal

$\theta_{1} \quad$ Ángulo entre los enlaces ab y bc

$\theta_{2} \quad$ Ángulo entre los enlaces ac

$\theta \quad$ Ángulo en sección transversal comprendido por el sector de los enlaces b tomando como vértice el centro de la circunferencia

$\varphi \quad$ Ángulo en sección transversal comprendido por el sector de los enlaces a y c tomando como vértice el centro de la circunferencia

Tabla A.14: Determinación diámetro inicial por minimización energética, SWNTs Armchair

\begin{tabular}{ll}
\hline$n, m$ & Entero que caracteriza un nanotubo $\mathrm{CH}(\mathrm{n}, \mathrm{m})$ \\
$\beta$ & Ángulo entre los enlaces a y la línea ZZ (parámetro independiente) \\
$\eta$ & $\begin{array}{l}\text { Ángulo entre los enlaces b y la línea ZZ (parámetro independiente) } \\
\text { Desplazamiento de los enlaces c en la dirección de la línea ZZ para cada celda } \\
\text { hexagonal }\end{array}$ \\
$d_{a}, d_{b}, d_{c}$ & $\begin{array}{l}\text { Longitudes de las proyecciones de los respectivos enlaces a,b,c sobre la sección } \\
\text { transversal }\end{array}$ \\
$\varphi_{a}, \varphi_{b}, \varphi_{c}$ & $\begin{array}{l}\text { Ángulos en sección transversal comprendidos por los respectivos sectores } \\
\text { tomando como vértice el centro de la circunferencia }\end{array}$ \\
$\theta_{a b}, \theta_{a c}, \theta_{b c}$ & $\begin{array}{l}\text { Ángulos entre los respectivos enlaces en la geometría inicial del SWNT } \\
n_{v}\end{array}$ \\
$k$ & $\begin{array}{l}\text { Número de nudos (átomos) contenidos en la banda unitaria } \\
\text { Iteración genérica del proceso iterativo }\end{array}$ \\
\hline
\end{tabular}

Tabla A.15: Determinación diámetro inicial por minimización energética, SWNTs Chiral 


\section{A.2. Índice de acrónimos}

Relacionamos a continuación los acrónimos más empleados en esta tesis, respetando sus iniciales en inglés debido a que así aparecen en la práctica totalidad de referencias consultadas.

\begin{tabular}{ll}
\hline AC & Armchair nanotubes \\
AFM & Atomic Force Microscope \\
BEM & Boundary Element Method \\
FEM & Finite Element Method \\
BSM & Bridging Scale Method \\
CH & Chiral nanotubes \\
CNTs & Carbon Nanotubes \\
DFT & Density Functional Theory \\
FEM & Finite Element Method \\
MD & Molecular Dynamics \\
MSM & Molecular Structural Mechanics \\
MM & Molecular Mechanics \\
CVD & Chemical Vapor Deposition \\
HAC & Hibrid Atomistic-Continuum Method \\
HREM & High Resolution Electron Microscope \\
L-J & Lennard-Jones potential \\
MS & Multi-Scale Simulation methods \\
MWNTs & Multi-Walled Carbon Nanotubes \\
QC & Quasi-Continuum Method \\
NEMS & Nano-Electro Mechanical Systems \\
QM & Quantum Mechanics \\
SEM & Scanning Electron Microscope \\
STM & Scanning Transmission Microscope \\
SVD & Singular Value Decomposition \\
S-W & Stone-Wales \\
SWNTs & Single-Walled Carbon Nanotubes \\
TB & Tight Binding Method \\
T-B & Tersoff-Brenner potential \\
TEM & Transmission Electron Microscope \\
VDW & Van der Waals \\
VGCF & Vapor Grown Carbon Fibers \\
ZZ & ZigZag nanotubes \\
\hline &
\end{tabular}





\section{Bibliografía}

[1] P. Ajayan, L. Schadler, C. Giannaris, and A. Rubio. Single-walled carbon nanotube-polymer composites: Strength and weakness. Advanced Materials, 12(10):750-753, 2000.

[2] R. Ansari, M. Mirnezhad, and S. Sahmani. An accurate molecular mechanics model for computation of size-dependent elastic properties of armchair and zigzag single-walled carbon nanotubes. Meccanica, pages 1-13, 2012.

[3] M. Ariza and M. Ortiz. Discrete dislocations in graphene. Journal of the Mechanics and Physics of Solids, 58(5):710-734, 2010.

[4] M. Ariza, M. Ortiz, and R. Serrano. Long-term dynamic stability of discrete dislocations in graphene at finite temperature. International Journal of Fracture, 166(1):215-223, 2010.

[5] M. Ariza, R. Serrano, J. Mendez, and M. Ortiz. Stacking faults and partial dislocations in graphene. Philosophical Magazine, 92(16):2004-2021, 2012.

[6] M. Arroyo and T. Belytschko. Nonlinear mechanical response and rippling of thick multiwalled carbon nanotubes. Phys. Rev. Lett., 91(21):215505, Nov 2003.

[7] T. Belytschko, S. P. Xiao, G. C. Schatz, and R. S. Ruoff. Atomistic simulations of nanotube fracture. Phys. Rev. B, 65(23):235430, Jun 2002.

[8] L. G. Brazier. On the flexure of thin cylindrical shells and other "thin"sections. Proc. Royal Society London A, 116(773):104-114, September 1927.

[9] M. Brcic, C. Marko, J. Brnic, D. Lanc, S. Krscansi, and G. Vukelic. Fe modelling of multiwalled carbon nanotubes. Estonian Journal of Engineering, 15(2):77-86, 2009.

[10] D. W. Brenner. Empirical potential for hydrocarbons for use in simulating the chemical vapor deposition of diamond films. Phys. Rev. B, 42(15):9458-9471, Nov 1990.

[11] G. Cao and X. Chen. The effect of the displacement increment on the axial compressive buckling behaviours of single-walled carbon nanotubes. Nanotechnology, 17(15):3844-3855, 2006.

[12] Y. Chan, N. Thamwattana, and J. M. Hill. Axial buckling of multi-walled carbon nanotubes and nanopeapods. European Journal of Solid Mechanics ASolids, 30:794-806, May 2011.

[13] K. Chandraseker and S. Mukherjee. An atomistic-continuum elastic rod model of carbon nanotubes. In J. F. ABEL and J. R. COOKE, editors, Proceedings of the 6th International Conference on Computation of Shell and Spatial Structures IASS-IACM 2008: "Spanning Nano to Mega", Ithaca, NY, USA, May 2008. Cornell University. 
[14] T. Chang and H. Gao. Size-dependent elastic properties of a single-walled carbon nanotube via a molecular mechanics model. Journal of the Mechanics and Physics of Solids, 51(6):1059 $-1074,2003$.

[15] T. Chang, J. Geng, and X. Guo. Prediction of chirality- and size-dependent elastic properties of single-walled carbon nanotubes via a molecular mechanics model. Proceedings of the Royal Society A, 462:2523-2540, 2006.

[16] T. Chang, G. Li, and X. Guo. Elastic axial buckling of carbon nanotubes via a molecular mechanics model. Carbon, 43:287-294, 2005.

[17] N. G. Chopra and A. Zettl. Measurement of the elastic modulus of a multi-wall boron nitride nanotube. Solid State Communications, 105(5):297 - 300, 1998.

[18] R. Chowdhury, C. Wang, and S. Adhikari. Low frequency vibration of multiwall carbon nanotubes with heterogeneous boundaries. Journal of Physics D: Applied Physics, 43:085405(8pp), February 2010.

[19] R. W. Clough and J. Penzien. Dynamics of Structures. McGraw-Hill, 1975.

[20] W. D. Cornell, P. Cieplak, C. I. Bayly, I. R. Gould, J. Kenneth M. Merz, D. M. Ferguson, D. C. Spellmeyer, T. Fox, J. W. Caldwell, and P. A. Kollman. A second generation force field for the simulation of proteins, nucleic acids, and organic molecules. Journal of the American Chemical Society, 117(19):5179-5197, 1995.

[21] C. F. Cornwell and L. T. Wille. Elastic properties of single-walled carbon nanotubes in compression. Solid State Communications, 101:555-558(4), February 1997.

[22] M. A. Crisfield. Non-linear finite element analysis of solids and structures: essentials, volume Vol I. Wiley \& Sons, 1991.

[23] M. A. Crisfield. Non-linear finite element analysis of solids and structures: advanced topics, volume Vol II. Wiley \& Sons, 1997.

[24] M. Dequesnes, S. Rotkin, and N. Aluru. Parameterization of continuum theories for single wall carbon nanotube switches by molecular dynamics simulations. Journal of Computational Electronics, 1(3):313-316, October 2002.

[25] J. Despres, E. Daguerre, and K. Lafdi. Flexibility of graphene layers in carbon nanotubes. Carbon, 33(1):87-89, 1995.

[26] M. S. Dresselhaus, G. Dresselhaus, and P. Avouris. Carbon Nanotubes: Synthesis, Structure, Properties, and Applications, volume 80 of Topics in Applied Physics. Springer Berlin, 2001. Varios autores.

[27] M. R. Falvo, G. J. Clary, R. M. Taylor, V. Chi, F. P. Brooks, S. Washburn, and R. Superfine. Bending and buckling of carbon nanotubes under large strain. Nature, 389(6651):582-584, October 1997.

[28] S. Fan, M. Chapline, N. Franklin, T. Tombler, A. Cassell, and H. Dai. Self-oriented regular arrays of carbon nanotubes and their field emission properties. Science, 283(5401):512-514, January 1999.

[29] C. A. Felippa. Nonlinear finite element methods. Notes for a graduate course offered at the University of Colorado (Boulder), 2001. 
[30] H. Goldstein. Mecánica Clásica. Ed. Reverté, 1990.

[31] T. Gould and D. A. Burton. A cosserat rod model with microstructure. New Journal of Physics, 8(137), August 2006.

[32] S. Govindjee and J. Sackman. On the use of continuum mechanics to estimate the properties of nanotubes. Solid State Communications, 110:227-230(4), 31 March 1999.

[33] T. Halicioglu. Stress calculations for carbon nanotubes. Thin Solid Films, 312(1-2):11-14, 1998.

[34] V. M. Harik. Mechanics of carbon nanotubes: applicability of the continuum-beam models. Computational Materials Science, 24(3):328 - 342, 2002.

[35] X. He, C. Qu, Q. Qin, and W. C.M. Buckling and postbuckling analysis of multi-walled carbon nanotubes based on the continuum shell model. International Journal of Structural Stability and Dynamics, 7(4):629-645, 2007.

[36] T. J. Healey. Material Symmetry and Chirality in Nonlinearly Elastic Rods. Mathematics and Mechanics of Solids, 7(4):405-420, 2002.

[37] E. Hernández, C. Goze, P. Bernier, and A. Rubio. Elastic properties of C and $B_{x} C_{y} N_{z}$ pomposite Nanotubes. Physical Review Letters, 80:4502-4505, May 1998.

[38] T. Hertel, R. E. Walkup, and P. Avouris. Deformation of carbon nanotubes by surface van der waals forces. Physical review B, 58(20):13870-13873, Nov 1998.

[39] Y. Hu, K. Liew, Q. Wang, X. He, and B. Yakobson. Nonlocal shell model for elastic wave propagation in single- and double-walled carbon nanotubes. Journal of the Mechanics and Physics of Solids, 56(12):3475-3485, December 2008.

[40] Y. Huang, J. Wu, and K. C. Hwang. Thickness of graphene and single-wall carbon nanotubes. Physical review B, 74(24):245413, 2006.

[41] A. Ibrahimbegovic and D. Markovic. Strong coupling methods in multi-phase and multi-scale modelling of inelastic behavior of heterogeneous structures. Computer methods in applied mechanics and engineering, 192:3089-3107, 2003.

[42] S. Iijima. Helical microtubules of graphitic carbon. Nature, 354:56-58, November 1991.

[43] S. Iijima, C. Brabec, A. Maiti, and J. Bernholc. Structural flexibility of carbon nanotubes. Journal of Chemical Physics, 104:2089-2092, Feb. 1996.

[44] H. Jiang, P. Zhang, B. Liu, Y. Huang, P. H. Geubelle, H. Gao, and K. C. Hwang. The effect of nanotube radius on the constitutive model for carbon nanotubes. Computational Materials Science, 28(3-4):429 - 442, 2003. Twelfth International Workshop on Computational Mechanics of Materials.

[45] Y. Jin and F. Yuan. Simulation of elastic properties of single-walled carbon nanotubes. Composites Science and Technology, 63:1507-1515(9), August 2003.

[46] J. E. Jones. On the Determination of Molecular Fields. I. From the Variation of the Viscosity of a Gas with Temperature. Proceedings of the Royal Society of London. Series A, 106(738):441$462,1924$. 
[47] J. E. Jones. On the Determination of Molecular Fields. II. From the Equation of State of a Gas. Proceedings of the Royal Society of London. Series A, 106(738):463-477, 1924.

[48] I. Kang, M. J. Schulz, J. H. Kim, V. Shanov, and D. Shi. A carbon nanotube strain sensor for structural health monitoring. Smart Materials and Structures, 15:737-748, April 2006.

[49] O. V. Kharissova and C. Robledo Jiménez. Sintesis de nanoestructuras de carbono mediante microondas. Ingenierías, VII(23), Abril-Junio 2004.

[50] A. Krishnan, E. Dujardin, T. W. Ebbesen, P. N. Yianilos, and M. M. J. Treacy. Young's modulus of single-walled nanotubes. Physical review B, 58(20):14013-14019, Nov 1998.

[51] S. Krylov, B. R. Ilic, D. Schreiber, S. Seretensky, and H. G. Craighead. Stability analysis of a curved microbeam actuated by a distributed electrostatic force. In J. F. ABEL and J. R. COOKE, editors, Proceedings of the 6th International Conference on Computation of Shell and Spatial Structures IASS-IACM 2008: “Spanning Nano to Mega”, Ithaca, NY, USA, May 2008. Cornell University.

[52] K. N. Kudin, G. E. Scuseria, and B. I. Yakobson. $C_{2} F$, BN, and C nanoshell elasticity from ab initio computations. Physical review B, 64(23):235406, 2001.

[53] C. Lanczos. The Variational Principles of Mechanics. Dover, New York, 1986. Reedición del original publicado en 1949 por University of Toronto Press.

[54] K. T. Lau, M. Chipara, H. Y. Ling, and D. Hui. On the effective elastic moduli of carbon nanotubes for nanocomposite structures. Composites Part B: Engineering, 35(2):95-101, 2004. Nanocomposites.

[55] T. A. Lauderdale and O. M. O'Reilly. On the restrictions imposed by non-affine material symmetry groups for elastic rods: application to helical substructures. European Journal of Mechanics - A/Solids, 26(4):701-711, July-August 2007.

[56] C. Lazaro. Formulación material y espacial del modelo geométricamente exacto de piezas alargadas. PhD thesis, Dept. MMCC y Teoría de Estructuras, Julio 2005.

[57] H. L. Lee and W. J. Chang. Dynamic modelling of a single-walled carbon nanotube for nanoparticle delivery. Proceedings of the Royal Society A, 467:860-868, June 2011.

[58] T. Lenosky, X. Gonze, M. Teter, and V. Elser. Energetics of negatively curved graphitic carbon. Nature, 355(6358):333-335, January 1992.

[59] C. Li and T. W. Chou. Elastic moduli of multi-walled carbon nanotubes and the effect of van der waals forces. Composites Science and Technology, 63(11):1517-1524, August 2003.

[60] C. Li and T. W. Chou. Single-walled carbon nanotubes as ultrahigh frequency nanomechanical resonators. Physical Review B, 68:073405(3pp), 2003.

[61] C. Li and T. W. Chou. A structural mechanics approach for the analysis of carbon nanotubes. International Journal of Solids and Structures, 40(10):2487-2499, May 2003.

[62] C. Li and T. W. Chou. Modeling of heat capacities of multi-walled carbon nanotubes by molecular structural mechanics. Materials Science E Engineering A, 409:140-144, 2005.

[63] C. Li and T. W. Chou. Quantized molecular structural mechanics modeling for studying the specific heat of single-walled carbon nanotubes. Physical Review B, 71:075409(6pp), 2005. 
[64] X. Li, W. Yang, and B. Liu. Bending Induced Rippling and Twisting of Multiwalled Carbon Nanotubes. Physical Review Letters, 98(20):205502, May 2007.

[65] C. Lim. On the truth of nanoscale for nanobeams based on nonlocal elastic stress field theory: equilibrium, governing equiation and static deflection. Applied Mathematics and Mechanics (English Edition), 31(1):37-54, 2012.

[66] J. Z. Liu, Q. Zheng, and Q. Jiang. Effect of bending instabilities on the measurements of mechanical properties of multiwalled carbon nanotubes. Phys. Rev. B, 67(7):075414, Feb 2003.

[67] W. K. Liu, E. G. Karpov, S. Zhang, and H. S. Park. An introduction to computational nanomechanics and materials. Computer Methods in Applied Mechanics and Engineering, 193(1720):1529 - 1578, 2004.

[68] Y. Liu and X. Chen. Continuum models of carbon nanotube-based composites using the boundary element method. Electronic Journal of Boundary Elements, 1(2), 2003.

[69] R. Livesley. Métodos Matriciales para el Cálculo de Estructuras. Ed. Blume, 1970.

[70] J. P. Lu. Elastic properties of carbon nanotubes and nanoropes. Phys. Rev. Lett., 79(7):12971300, Aug 1997.

[71] J. P. Lu. Elastic properties of single and multilayered nanotubes. Journal of Physics and Chemistry of Solids, 58(11):1649 - 1652, 1997.

[72] C. Luo and O. M. O'Reilly. On the material symmetry of elastic rods. Journal of Elasticity, 60(1):35-56, April 2000.

[73] D. Markovic and A. Ibrahimbegovic. On micro-macro interface conditions for micro scale based fem for inelastic behavior of heterogeneous materials. Computer methods in applied mechanics and engineering, 193:5503-5523, 2004.

[74] M. Meo and M. Rossi. Prediction of young's modulus of single wall carbon nanotubes by molecular-mechanics based finite element modelling. Composites Science and Technology, 66(11-12):1597 - 1605, 2006.

[75] R. Merli, C. Lázaro, S. Monleón, and A. Domingo. A molecular structural mechanics model applied to the static behavior of single-walled carbon nanotubes: New general formulation. Computers $\mathcal{E}$ structures, http://dx.doi.org/10.1016/j.compstruc.2012.11.023 (In press), 2012.

[76] A. K. Naieni, P. Yaghoobi, and A. Nojeh. Structural deformations and current oscillations in armchair-carbon nanotube cross devices: a theoretical study. Journal of Physics D: Applied Physics, 44:085402(7pp), February 2011.

[77] T. Natsuki and M. Endo. Stress simulation of carbon nanotubes in tension and compression. Carbon, 42(11):2147-2151, 2004.

[78] T. Natsuki, K. Tantrakarn, and M. Endo. Prediction of elastic properties for single-walled carbon nanotubes. Carbon, 42(1):39-45, 2004.

[79] G. M. Odegard, T. S. Gates, L. M. Nicholson, and K. E. Wise. Equivalent-continuum modeling of nano-structured materials. Composite Science and Technology, 62(14):1869-1880, 2002.

[80] M. O’Keeffe, G. B. Adams, and O. F. Sankey. Predicted new low energy forms of carbon. Phys. Rev. Lett., 68(15):2325-2328, Apr 1992. 
[81] A. Pantano, D. M. Parks, and M. C. Boyce. Mechanics of deformation of single- and multiwall carbon nanotubes. Journal of Mechanics Physics of Solids, 52:789-821, Apr. 2004.

[82] R. Parnes and A. Chiskis. Buckling of nano-fibre reinforced composites: a re-examination of elastic buckling. Journal of the Mechanics and Physics of Solids, 50(4):855-879, April 2002.

[83] S. Paulson, M. R. Falvo, N. Snider, A. Helser, T. Hudson, A. Seeger, R. M. Taylor, R. Superfine, and S. Washburn. In situ resistance measurements of strained carbon nanotubes. Applied Physics Letters, 75(19):2936-2938, 1999.

[84] S. Pellegrino. Structural computations with the singular value decomposition of the equilibrium matrix. International Journal of Solids and Structures, 30(21):3025-3035, 1993.

[85] S. Pellegrino and C. Calladine. Matrix analysis of statically and kinematically indeterminate frameworks. International Journal of Solids and Structures, 22(4):409-428, 1986.

[86] Q. Pham, S. C. Yoon, C. H. Bok, and H. S. Kim. Multi-scale modelling scheme for carbon nanotube reinforced metal matrix composites. Key Engineering Materials, 345-346:1261$1264,2007$.

[87] R. B. Pipes, P. Hubert, J.-P. Salvetat, and L. Zalamea. Flexural deflection as a measure of van der waals interaction forces in the cnt array. Composites Science and Technology, 66(9):1125 - 1131, 2006. Nanocomposites.

[88] V. N. Popov, V. E. van Doren, and M. Balkanski. Elastic properties of single-walled carbon nanotubes. Physical Review B, 61:3078-3084, Jan. 2000.

[89] D. H. Robertson, D. W. Brenner, and J. W. Mintmire. Energetics of nanoscale graphitic tubules. Phys. Rev. B, 45(21):12592-12595, Jun 1992.

[90] A. Rochefort, P. Avouris, F. Lesage, and D. R. Salahub. Electrical and mechanical properties of distorted carbon nanotubes. Phys. Rev. B, 60(19):13824-13830, Nov 1999.

[91] C. Q. Ru. Effective bending stiffness of carbon nanotubes. Physical Review B, 62:9973-9976, Oct. 2000.

[92] C. Q. Ru. Elastic buckling of single-walled carbon nanotube ropes under high pressure. Physical Review B, 62(15):10405-10408, Oct 2000.

[93] J. P. Salvetat, G. A. D. Briggs, J. M. Bonard, R. R. Bacsa, A. J. Kulik, T. Stöckli, N. A. Burnham, and L. Forró. Elastic and shear moduli of single-walled carbon nanotube ropes. Phys. Rev. Lett., 82(5):944-947, Feb 1999.

[94] D. Srivastava, M. Menon, and K. Cho. Nanoplasticity of single-wall carbon nanotubes under uniaxial compression. Physical Review Letters, 83(15):2973-2976, October 1999.

[95] J. Tersoff. New empirical model for the structural properties of silicon. Phys. Rev. Lett., 56(6):632-635, Feb 1986.

[96] J. Tersoff and R. S. Ruoff. Structural properties of a carbon-nanotube crystal. Phys. Rev. Lett., 73(5):676-679, Aug 1994.

[97] A. Thess, R. Lee, P. Nikolaev, H. Dai, J. Petit, C. Robert, and et al. Cristaline ropes of metallic carbon nanotubes. Science, 273:483-487, 1996.

[98] R. Togores and C. Otero. Programación en AutoCAD con Visual LISP. McGraw-Hill, 2003. 
[99] M. M. J. Treacy, T. W. Ebbesen, and J. M. Gibson. Exceptionally high young's modulus observed for individual carbon nanotubes. Nature, 381(20):678-680, June 1996.

[100] K. Tserpes and P. Papanikos. Finite element modeling of single-walled carbon nanotubes. Composites Part B: engineering, 36:468-477, 2005.

[101] Z. C. Tu and Z. C. Ou-Yang. Single- and multi-walled carbon nanotubes viewed as elastic tubes with young's moduli dependent on layer number. Physical Review B, 65:233407, 2002.

[102] C. Y. Wang and L. C. Zhang. An elastic shell model for characterizing single-walled carbon nanotubes. Nanotechnology, 19(19):195704 (6pp), 2008.

[103] L. F. Wang, Q. Zheng, J. Z. Liu, and Q. Jiang. Size dependence of the thin-shell model for carbon nanotubes. Physical review letters, 95(10):105501 (4pp), September 2005.

[104] L. F. Wang and Q. S. Zheng. Instabilities of Carbon Nanotubes Studied Using a Hybrid Atom/Continuum Approach. IUTAM Symposium on Mechanical Behavior and MicroMechanics of Nanostructured Materials, volume 144. Springer Netherlands, 2007.

[105] M. S. Wang, I. Kaplan-Ashiri, X. L. Wei, R. Rosentsveig, H. D. Wagner, R. Tenne, and L. M. Peng. In situ tem measurements of the mechanical properties and behavior of ws 2 nanotubes. Nano Research, 1(1):22-31, 2008.

[106] Q. Wang. Effective in-plane stiffness and bending rigidity of armchair and zigzag carbon nanotubes. International Journal of Solids and Structures, 41(20):5451-5461, October 2004.

[107] Q. Wang and V. K. Varadan. Stability analysis of carbon nanotubes via continuum models. Smart Materials and Structures, 14(1):281-286, 2005.

[108] X. Wang, X. Wang, and J. Xiao. A non-linear analysis of the bending modulus of carbon nanotubes with rippling deformations. Composite Structures, 69(3):315 - 321, 2005.

[109] Y. Wang, X. X. Wang, and X. Ni. Atomistic simulation of the torsion deformation of carbon nanotubes. Modelling and Simulation in Materials Science and Engineering, 12:1099-1107, August 2004.

[110] Y. Wang, X. xi Wang, X. gui Ni, and H. an Wu. Simulation of the elastic response and the buckling modes of single-walled carbon nanotubes. Computational Materials Science, 32(2): $141-146,2005$.

[111] B. WenXing, Z. ChangChun, and C. WanZhao. Simulation of young's modulus of singlewalled carbon nanotubes by molecular dynamics. Physica B: Condensed Matter, 352(1-4):156 - 163, 2004.

[112] E. W. Wong, P. E. Sheehan, C. Lieber, and M. Nanobeam mechanics: Elasticity, strength, and toughness of nanorods and nanotubes. Science, 277(5334):1971-1975, September 1997.

[113] J. Wuite and S. Adali. Deflection and stress behaviour of nanocomposite reinforced beams using a multiscale analysis. Composite structures, 71:388-396, 2005.

[114] J. Xiao, B. Gama, J. Gillespie, and Jr. An analytical molecular structural mechanics model for the mechanical properties of carbon nanotubes. International Journal of Solids and Structures, 42(11-12):3075-3092, 2005.

[115] B. I. Yakobson, C. J. Brabec, and J. Bernholc. Nanomechanics of carbon tubes: Instabilities beyond linear response. Phys. Rev. Lett., 76(14):2511-2514, Apr 1996. 
[116] Y. Yan, W. Wang, and L. Zhang. Applied multiscale method to analysis of nonlinear vibration for double-walled carbon nanotubes. Applied Mathematical Modelling, 35:2279-2289, 2011.

[117] M. F. Yu. Fundamental mechanical properties of carbon nanotubes: Current understanding and the related experimental studies. Journal of Engineering Materials and Technology, 126(3):271-278, 2004.

[118] M. F. Yu, B. S. Files, S. Arepalli, and R. S. Ruoff. Tensile loading of ropes of single wall carbon nanotubes and their mechanical properties. Phys. Rev. Lett., 84(24):5552-5555, Jun 2000 .

[119] M. F. Yu, O. Lourie, M. J. Dyer, K. Moloni, T. F. Kelly, and R. S. Ruoff. Strength and Breaking Mechanism of Multiwalled Carbon Nanotubes Under Tensile Load. Science, 287(5453):637640, 2000.

[120] M. Zaeri, S. Ziaei-Rad, A. Vahedi, and F. Karimzadeh. Mechanical modelling of carbon nanomaterials from nanotubes to buckypaper. Carbon, 48:3916-3930, June 2010.

[121] H. Zhang, L. Wang, and J. Wang. Computer simulation of buckling behavior of doublewalled carbon nanotubes with abnormal interlayer distances. Computational Materials Science, 39(3):664-672, 2007.

[122] P. Zhang, Y. Huang, P. H. Geubelle, P. A. Klein, and K. C. Hwang. The elastic modulus of single-wall carbon nanotubes: a continuum analysis incorporating interatomic potentials. International Journal of Solids and Structures, 39(13-14):3893 - 3906, 2002.

[123] P. Zhang, P. E. Lammert, and V. H. Crespi. Plastic deformations of carbon nanotubes. Phys. Rev. Lett., 81(24):5346-5349, Dec 1998.

[124] Q. Zhao, M. B. Nardelli, and J. Bernholc. Ultimate strength of carbon nanotubes: A theoretical study. Phys. Rev. B, 65(14):144105, Mar 2002. 